\title{
FY04 Engineering Technology Reports
}

\section{Techuology" Base}
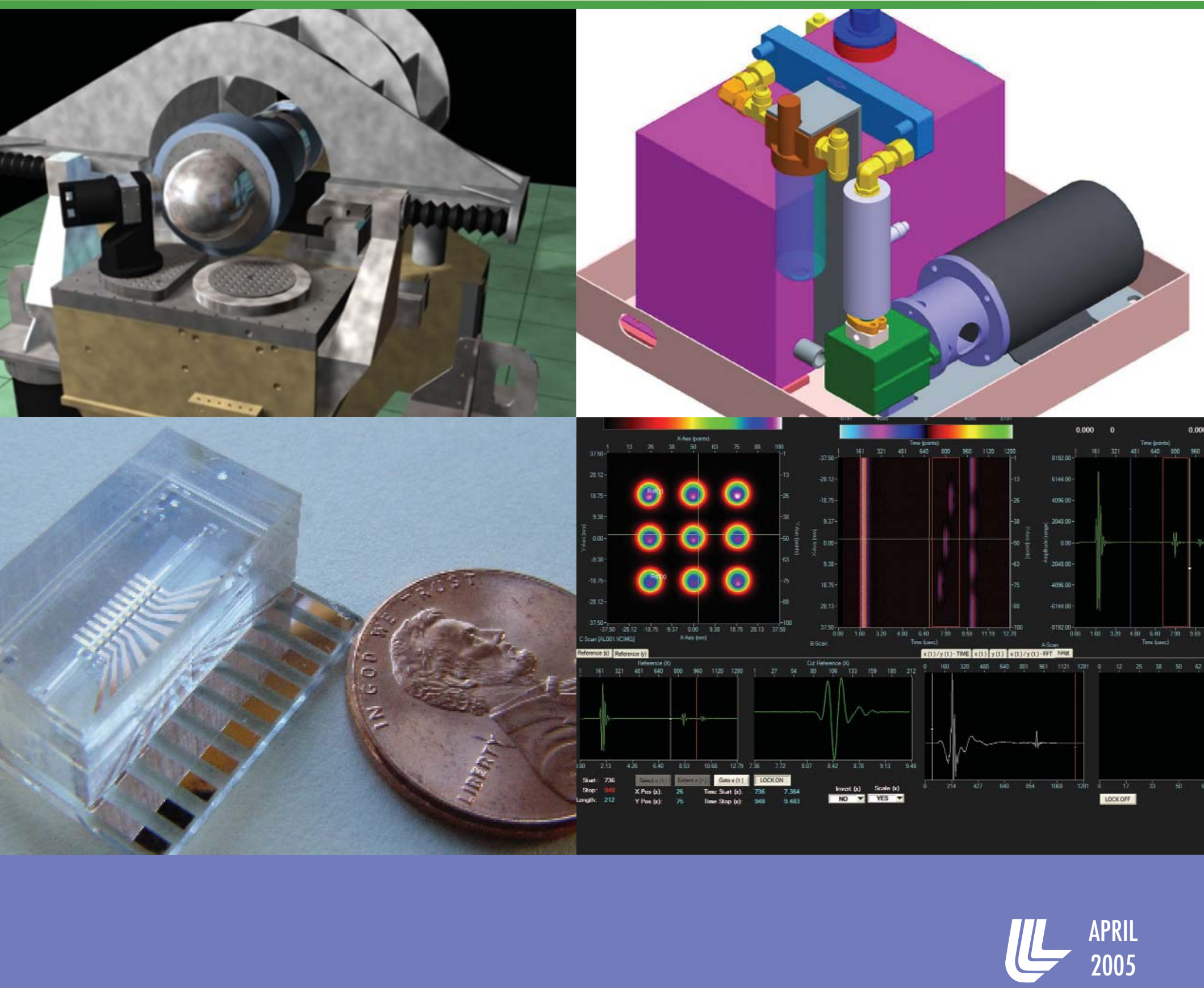

Lawrence Livermore National Laboratory 


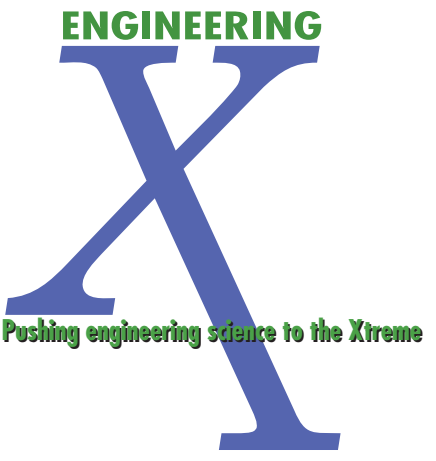

\section{Acknowledgments}

Scientific Editing

Camille Minichino

Graphic Design

Irene J. Chan

Art Production/Layout

Jeffrey Bonivert

Lucy Dobson

Kathy J. McCullough

Debbie A. Ortega

\section{Cover:}

Graphics representing technology-base projects from Engineering's five Centers and other technologies. 


\section{EY04 Engineering Technology Reports}

\section{Technalegry Babe}

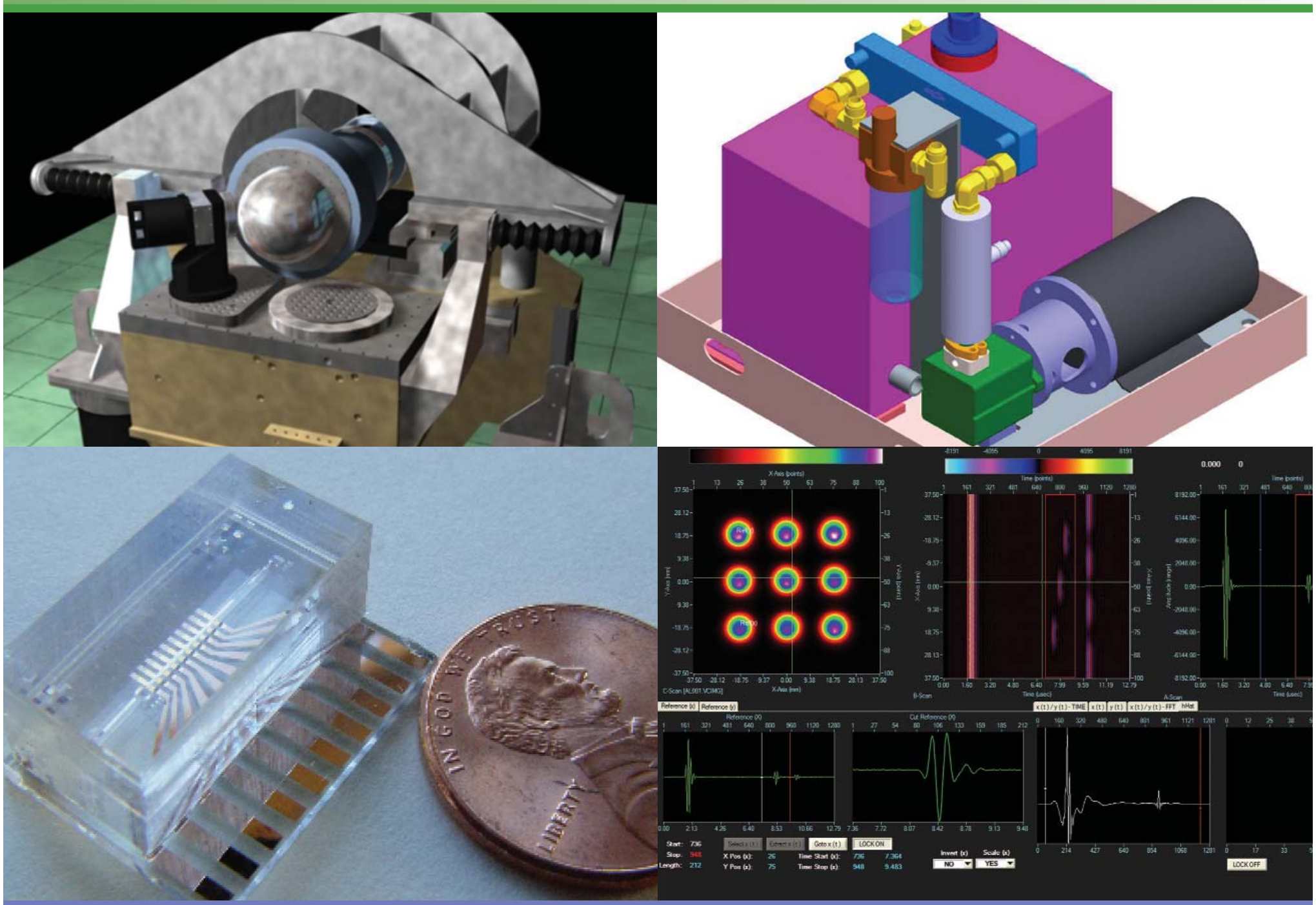

나 2015 


\section{Contents}

\section{Introduction}

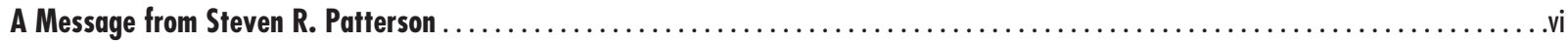

\section{Center for Computational Engineering}

DYNA3D Enhancement and Support

Jerry I. Lin

FEA Visualization and Data Management

Elsie M. Pierce

NIKE3D Enhancement and Support

Michael A. Puso

E3D Modifications for Engineering Applications

David H. Chambers

Engineering Visualization Theater

Michael D. Loomis

Enhanced Fluid Dynamics Modeling Capability

Kambiz Salari

Enhanced Fluid Dynamics Modeling Capability for Endovascular Flows

Jason Ortega

Modeling Initiation in Exploding Bridgewire Detonators

Constantine A. Hrousis

Modeling Production Plant Forming Processes

Moon Rhee

Modeling Rarefied Gas Flows with Direct Simulation Monte Carlo

Todd Weisgraber

Sensitivity Analysis in Modeling the Fate and Transport of Chemical and Biological Agents

Lee Greer Glascoe

Technologies for Structural Damage Analysis

Charles R. Noble

Center for Microtechnology and Nanotechnology

Contact Stress Distribution Microsensor Array and Optical Force Probe

Jack Kotovsky 
Direct Wafer Bonding of Gallium-Arsenide to Sapphire

Gregory A. Cooper

Electrochemical Detection of Biological Pathogens

David Sopchak

In-Situ Characterization Tools for ECR Plasma Etching

Rebecca Welty ...

Microfluidic Backbone and Components Using New Fabrication Capability for CBNP Applications

Robin Miles.

Miniature Multichannel Fiber-Optic Signal Conditioner

Michael D. Pocha

Three-Dimensional Microlithography System

Vincent Malba . .

\section{Center for Nondestructive Characterization}

Application of Nondestructive Evaluation to Finite-Element Analysis Coupling for Numerical Analysis

Edwin J. Kokko

Neutron DR/CT Upgrade at McClellan Nuclear Radiation Center

Daniel J. Schneberk

Quantitative Characterization of Mesoscale Objects

Amy M. Waters

Three-Dimensional Rendering of High-Frequency Ultrasonic Data

Steven E. Benson

Ultrasonic Calibration Test Phantom

Sean K. Lehman

\section{Center for Precision Engineering}

Hydraulic Power Unit for Hydrostatic Bearings

David J. Hopkins

Noncontact Diamond Tool Metrology

Jeremy J. Kroll

Ultra-Precision Machine Spindle Using Porous Ceramic Bearings

Paul Geraghty

\section{Center for Complex Distributed Systems}

Air Valve Truck-Stopping Device

Mark Strauch 
Analysis of Emerging Mote Technology as a Distributed Sensor Platform

Kenneth M. Masica

Cooperative Discrimination Sensor: Detecting and Tracking Human Activity

Richard R. Leach, Jr.

Long-Range Ultra-Wideband Radio-Frequency Identification

Farid U. Dowla

Miniature Echelle Grating Spectrometer Cartridge

Joel Bowers

\section{Other Technologies}

Beryllium Surface Cleaning Process

Thomas M. Vercelli

Camouflaged Object Recognition

Chi Yung Fu

Demonstration of Armored Vehicle Technology

Steven J. DeTeresa

Experimental Verification of Correlation-Based Wavefront Sensing

Lisa A. Poyneer

Real-Time Speckle Imaging for Video Surveillance

Carmen J. Carrano

Field-Programmable-Gate-Array-Based System for Network Operations

J. Steven Blessing

Flexible Testbed for Evaluation of Ultra-Wideband (UWB) Radios

Alex Spiridon

Laser Peening Study for Reduction in Hydrogen Permeation in a Titanium Alloy

Tania Zaleski

Hyperspectral Processing Using FPGAs and DSPs

Erik David Jones

Magnetic Matching of Ultra-Compact Marx Generator

Jay B. Javedani

Multipoint Arc Initiation

David Steich

Nano-Mechanical Characterization Tools for In-Situ Deformation and Morphology of Materials

Scott Groves 
Solid-State Replacements for Hydrogen Thyratrons

Phillip A. Arnold ...

Transient Sampling

Craig S. Halvorson 104

Author Index .108 


\title{
A Message from
}

\author{
Steven R. Patterson, \\ Associate Director for Engineering
}

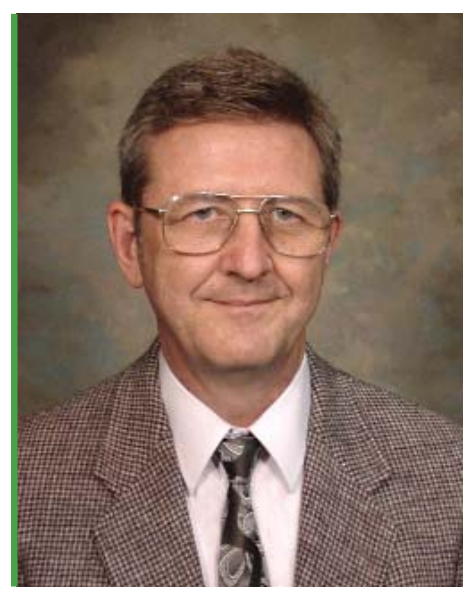

awrence Livermore National Laboratory's Engineering

Directorate has two primary discretionary avenues for its investment in technologies: the Laboratory Directed Research and Development (LDRD) program and the "Tech Base" program. This volume summarizes progress on the projects funded for technology-base efforts in FY2004.

The Engineering Technical Reports exemplify Engineering's more than 50-year history of researching and developing (LDRD), and reducing to practice (technology-base) the engineering technologies needed to support the Laboratory's missions. Engineering has been a partner in every major program and project at the Laboratory throughout its existence, and has prepared for this role with a skilled workforce and technical resources. This accomplishment is well summarized by Engineering's mission: "Enable program success today and ensure the Laboratory's vitality tomorrow."

LDRD is the vehicle for creating those technologies and competencies that are cutting edge. These require a significant level of research or contain some unknown that needs to be fully understood. Tech Base is used to apply those technologies, or adapt them to a Laboratory need. The term commonly used for Tech Base projects is "reduction to practice."

Tech Base projects effect the natural transition to reductionto-practice of scientific or engineering methods that are well understood and established. They represent discipline-oriented, core competency activities that are multi-programmatic in application, nature, and scope.

The objectives of technology-base funding include:

- the development and enhancement of tools and processes to provide Engineering support capability, such as code maintenance and improved fabrication methods;
- support of Engineering science and technology infrastructure, such as the installation or integration of a new capability;

- support for technical and administrative leadership through our technology Centers;

- the initial scoping and exploration of selected technology areas with high strategic potential, such as assessment of university, laboratory, and industrial partnerships.

Engineering's five Centers, in partnership with the Division Leaders and Department Heads, focus and guide longer-term investments within Engineering. The Centers attract and retain top staff, develop and maintain critical core technologies, and enable programs. Through their technology-base projects, they oversee the application of known engineering approaches and techniques to scientific and technical problems. The Centers and their Directors are as follows:

- Center for Computational Engineering:

Robert M. Sharpe

- Center for Microtechnology and Nanotechnology:

Raymond P. Mariella, Jr.

- Center for Nondestructive Characterization:

Harry E. Martz, Jr.

- Center for Precision Engineering:

Keith Carlisle

- Center for Complex Distributed Systems:

Gregory J. Suski, Acting Director

\section{FY2004 Center Highlights}

The Center for Computational Engineering orchestrates the research, development and deployment of software technologies 
to aid in many facets of LLNL's engineering mission. Computational engineering has become a ubiquitous component throughout the engineering discipline. Current activities range from tools to design the next generation of mixed-signal chips (systems on a chip) to full-scale analysis of key DOE and DoD systems.

Highlights of the Center's technology-base projects for FY2004 include enhancements, modifications, verification, and validation of engineering simulation tools and capabilities; progress in visualization and data management tools; and extensions of our competence in sensitivity and structural damage analysis. The Center has offered a real-world computing capability that opens the door to solving a wide variety of fluid/solid interaction problems in transportation, aerospace, and infrastructure settings.

The mission of the Center for Microtechnology and Nanotechnology is to invent, develop, and apply micro- and nanotechnologies to support LLNL missions in Stockpile Stewardship, Homeland Security, Nonproliferation, and other programs. The Center's capabilities cover materials, devices, instruments, and systems that require microfabricated components, including microelectromechanical systems (MEMS), electronics, photonics, microstructures, and microactuators. Center staff have achieved considerable national recognition for the successes demonstrated in Chem-Bio National Security Program instrumentation, supported by the DOE and the Defense Intelligence Agency.

Our FY2004 projects include the extension of our capabilities and tools for bonding and etching processes; advances in pathogen detection; implementation of a miniature multichannel signal conditioner; and upgrades to our capabilities in microlithography.

The Center for Nondestructive Characterization advances, develops and applies nondestructive characterization (NDC) measurement technology to significantly impact the manner in which LLNL inspects, and through this, designs, fabricates, and refurbishes systems and components. The Center plays a strategic and vital role in the reduction-to-practice of scientific and engineering NDC technologies, such as electromagnetic waves (infrared, microwave, visible and $\mathrm{x}$ rays), acoustics, and particles (e.g., protons) for imaging, to allow Engineering in the near-term to incorporate these technologies into LLNL and DOE programs.

This year's technology-base projects include the quantitative characterization of mesoscale objects; advances in ultrasonic techniques and numerical analysis; and the upgrade of neutron DR/CT at the McClellan Nuclear Radiation Center at the University of California, Davis.
The Center for Precision Engineering advances LLNL's highprecision capabilities in manufacturing, dimensional metrology and assembly, to meet the future needs of LLNL and DOE programs. Precision engineering is a multi-disciplinary systems approach to achieve an order of magnitude greater accuracy than currently achievable. The Center's core technologies are essential to the Laboratory because they will reduce the amount of research and development required to build the next generation of instruments and machine tools. By using proven technology, they also reduce the risk, the lead and design times, and the time to complete.

Highlights for Precision Engineering's FY2004 technologybase projects include advances in machine spindle dynamics, an ultrasonic beryllium surface cleaning process, a hydraulic power unit; and noncontact diamond tool metrology.

The Center for Complex Distributed Systems exploits emerging information technologies to develop unique communications related to data gathering, advanced signal processing, and new methodologies for assimilating measured data with computational models in data-constrained simulations of large systems. These technology-base projects are delivering application-ready tools into the hands of engineers supporting programs, and thus serve a critical link in transitioning from research to practice.

Current technology-base projects include an air valve truck-stopping device; the analysis of wireless mote technology as a sensor platform; discrimination sensors for detecting and tracking human activity; UWB RF identification; and an echelle grating spectrometer.

In FY2004, other technologies also contributed to Engineering's progress in camouflaged object recognition; armored vehicle technology; wavefront sensing; speckle imaging; FPGA systems; UWB radios; hydrogen permeation; magnetic matching of an ultra-compact Marx generator; multipoint arc initiation; characterization tools for in-situ deformation and morphology of materials; hydrogen thyratrons; and a transient sampling data recorder.

\section{Science-Based Engineering}

Our five Centers develop the key engineering technologies that make Laboratory programs successful. They provide the mechanism by which Engineering can help programs attract funding, while pioneering the technologies that will sustain long-term investment.

Our Centers, with staff who are full partners in Laboratory programs, integrate the best of mechanical and electronics engineering, creating a synergy that aids Engineering's mission, and helps turn the impossible into the doable. 

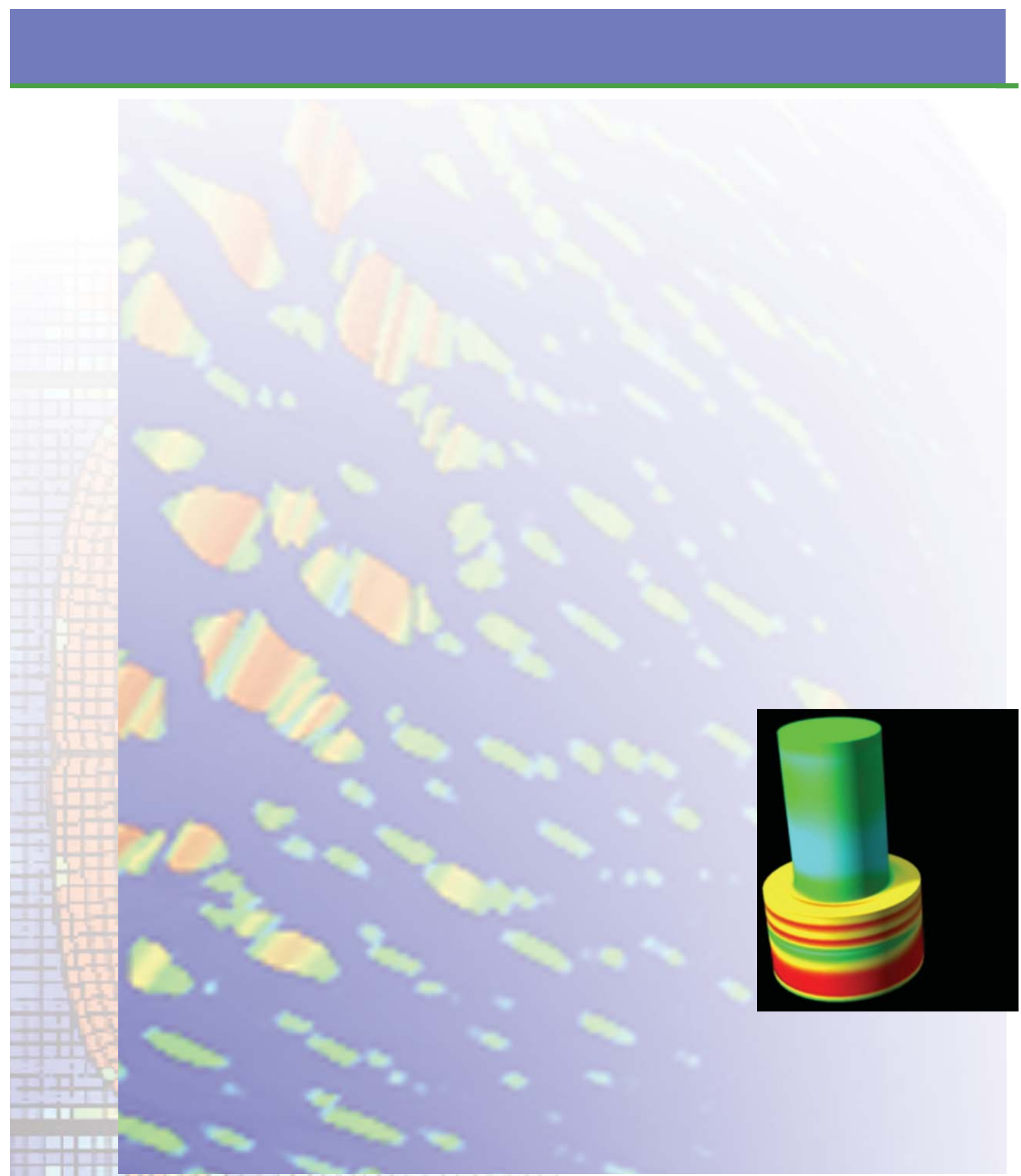

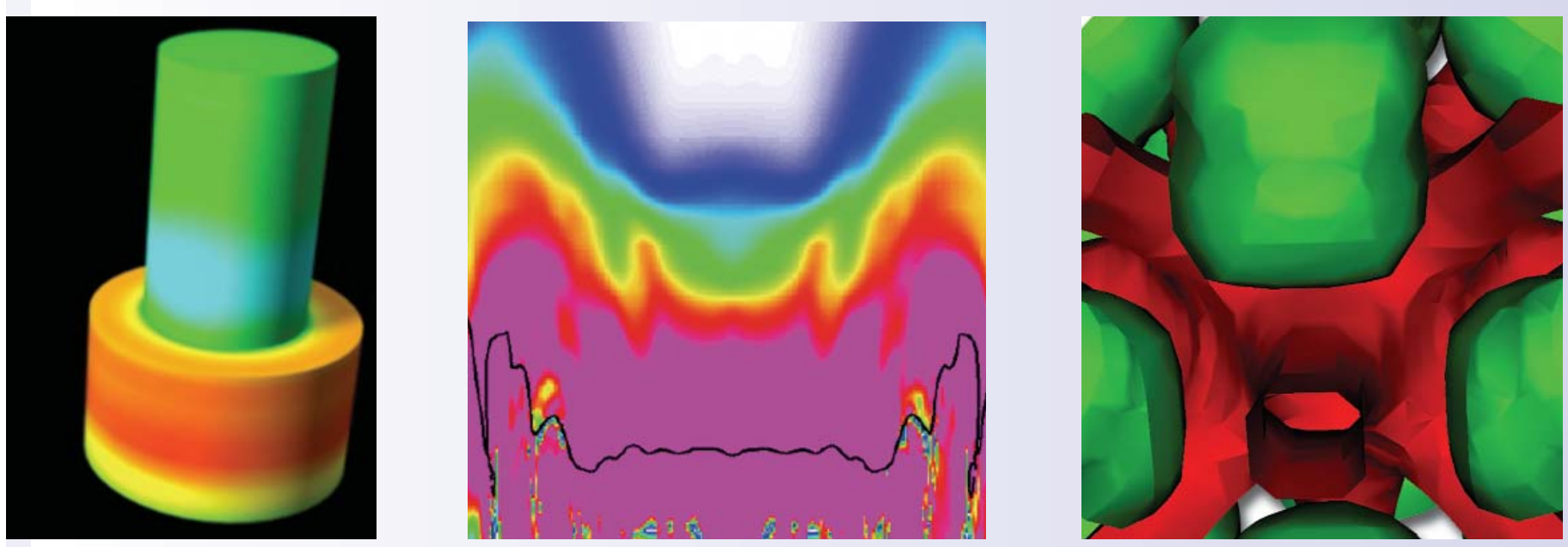

Center for
Computational Engineering 


\section{DYNA3D Enhancement and Support}

he explicit finite-element code

DYNA3D is one of the workhorse tools used by LLNL analysts for its ability to handle the nonlinear behaviors of structures/continuum, especially under fast transient loads.

\section{Project Goals}

This project undertakes the deployment of user-requested features, general technical support of users, and document update for DYNA3D. It also supports LLNL's collaborators program and the distribution of software to DOE's Energy Science and Technology Software Center. Together, these activities are needed to retain DYNA3D as a major simulation capability.

\section{Relevance to LLNL Mission}

New functionalities, maintenance, and technical support are essential to analysts contributing to ongoing projects in many LLNL programs. Many of these projects involve the Laboratory's collaboration with other institutions and federal agencies, such

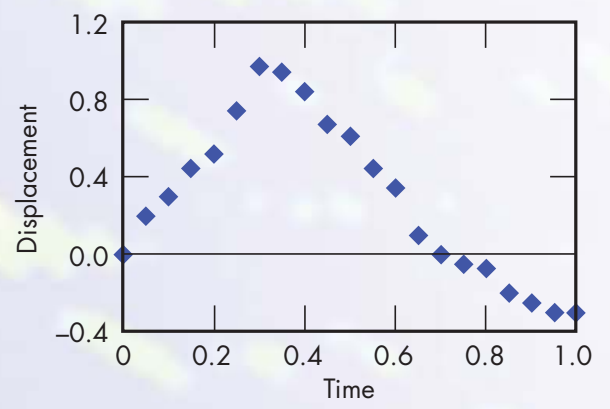

Figure 1. Sample of user-prescribed displacement input.

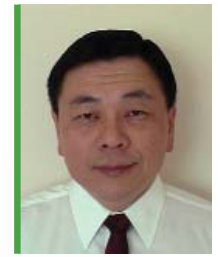

For more information contact Jerry I. Lin (925) 423-0907, lin5@|ln..gov

as Los Alamos National Laboratory, the Department of Homeland Security, and the Bureau of Reclamation. DYNA3D is a premier capability for simulating nonlinear, transient response of solids and structures.

\section{FY2004 Accomplishments and Results}

In computational structural analysis, a user-specified displacement history over portions(s) of the structure is a common way of imposing external loading functions. This is especially true in simulating a test procedure or during a verification/validation study. Due to DYNA3D's velocity-based formulation, such prescribed displacements must be converted to velocities for proper incorporation. The conventional assumption of linear variation between given displacement data leads to step-like, piecewise-constant velocity histories. Such velocity discontinuities can in turn cause excessive artificial high-frequency responses, commonly known as noise, in the analysis results.

The new algorithm uses the central difference operator to obtain discrete velocity

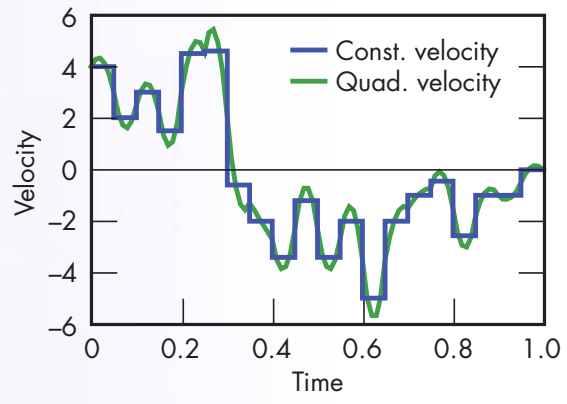

Figure 2. Velocity histories derived from the prescribed displacements by different interpolations. The feature implemented provides the smooth, quadratic velocities. 


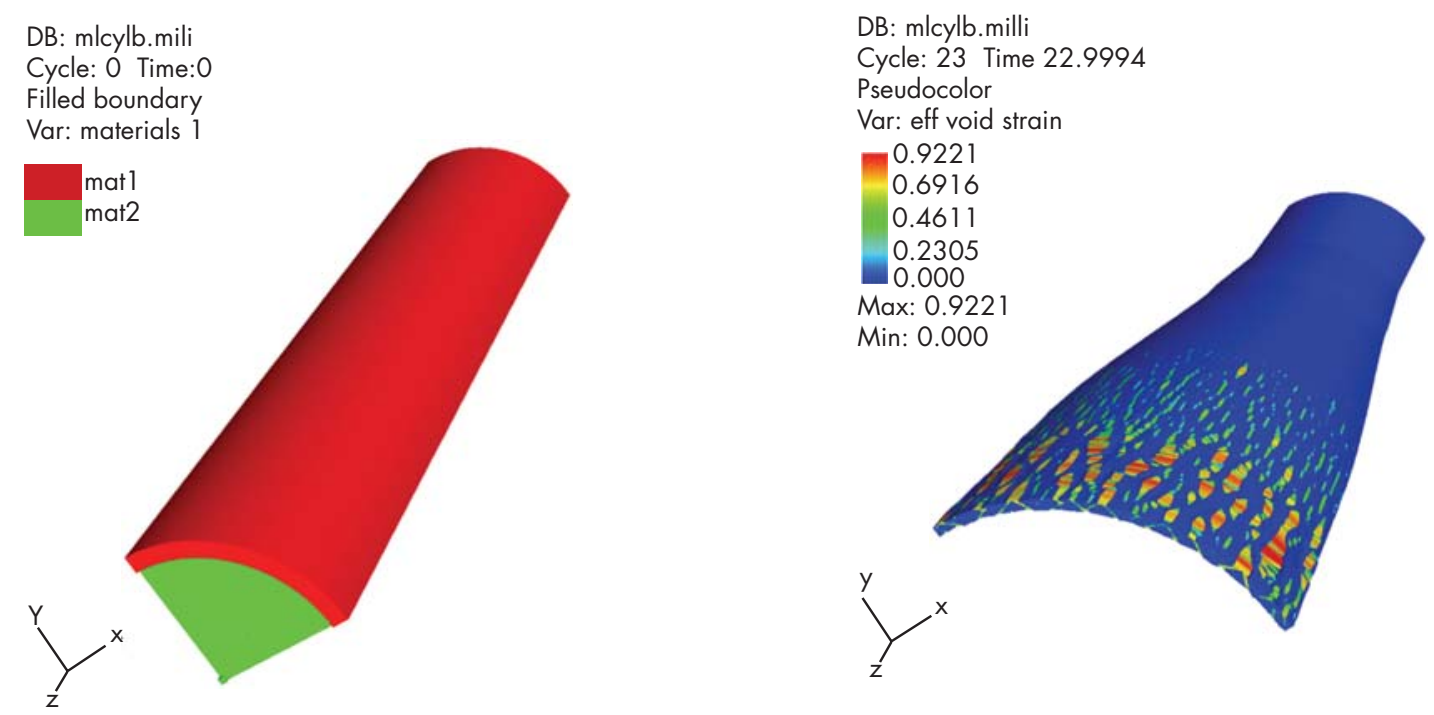

Figure 3. A cylindrical metal tube (red) with detonated HE (green). The detonation wave moves in the negative z direction. The figure shows the development of shear bands (localized regions of large deformation) and fractures. This simulation used the 3-D anisotropic fracture model MARFRAC, which was coupled to a Steinberg-Guinan plasticity model.

data for times of a given displacement. These generated velocity data, along with the user-specified displacements, afford a higher-order interpolation of velocity histories. The interpolated velocity history is continuous and piecewise-quadratic in time. It also yields the exact displacements at the user-specified points. Figure 1 shows a sample of user-specified displacement history. The comparison between corresponding piecewise-constant and piecewisequadratic velocities is shown in Fig. 2.

Two material models were modified to improve their performance or capability. The elastic-plastic material with oriented cracks was modified to avoid undesirable residual tensile stress and spurious surface loads. A 3-D anisotropic fracture model (MARFRAC) was coupled to the Steinberg-Guinan plasticity model. The model was tested and incorporated into the main DYNA3D software. An example calculation using the new model, the HEdriven expansion and fracturing of a metal cylinder, is shown in Fig. 3. This finite-element mesh contains approximately 10 million elements and was run with ParaDyn, a parallel processing code based on DYNA3D.

Adoption of the Mili I/O library has lent unprecedented flexibility to DYNA3D's visualization output. Many results and parameters that could not be included in the old output format have become available for inclusion in the Mili output database. Because of the large variety of available state variables and parameters, we elect to add them according to user requests. In FY2004, we also added nodal reaction forces and current volume/pressure for closed volumes in the DYNA3D output database.

\section{FY2005 Proposed Work}

Due to the varying demands of user support and user-requested feature addition, our typical planning strategy is to identify a set of logical "next steps" for feature deployment. For next year these features include:

1. Adding surface-related quantities to the visualization output database upon full deployment of the surface class capability in the Griz visualizer. This new class will enable many additional state variables and parameters to be included in the output database.

2. A more consistent and robust algorithm for stiffness-proportional damping to replace the existing approach. Its effect on the system stability limit will be fully considered in the time-step calculation.

3. Continuation of coding of revised algorithms for contact detection and interference resolution. This work focuses on the interaction between segment pairs, instead of the existing node and segment approach. 


\section{FEA Visualization and Data Management}

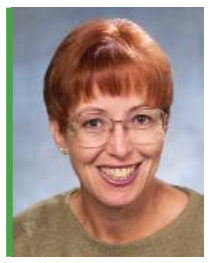

For more information contact Elsie M. Pierce

(925) 422-4063, pierce5@lnl.gov

ne component of our effort is devoted to the ongoing maintenance and enhancement of the GRIZ finite-element postprocessor and the Mili I/O library. GRIZ is our primary tool for visualizing finite-element analysis results on 3-D unstructured grids. The Mili I/O library provides the primary data path between simulation codes and GRIZ.

The value of simulation ultimately rests on how much useful information the analysts can derive from their numerical results. This means visual representations and interrogations of their data. GRIZ calculates and displays derived variables for multiple codes, most importantly DYNA3D and NIKE3D. GRIZ provides modern 3-D visualization techniques such as isocontours and isosurfaces, cutting planes, vector field display, and particle traces. GRIZ also incorporates the ability to animate all representations over time. A variety of simulation data can be passed from the simulation codes using the Mili database representation.

\section{Project Goals}

This project's overarching goal is to keep these tools functional and responsive to the needs of LLNL's engineering analysts.

\section{Relevance to LLNL Mission}

Our efforts with GRIZ and Mili provide important user interfaces to LLNL's nonlinear solid mechanics simulation capabilities, and facilitate their use by dozens of analysts.

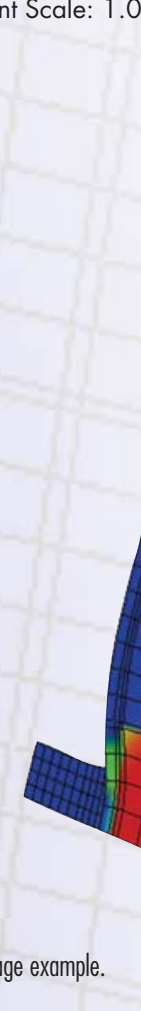

Figure 1. Damage example. 


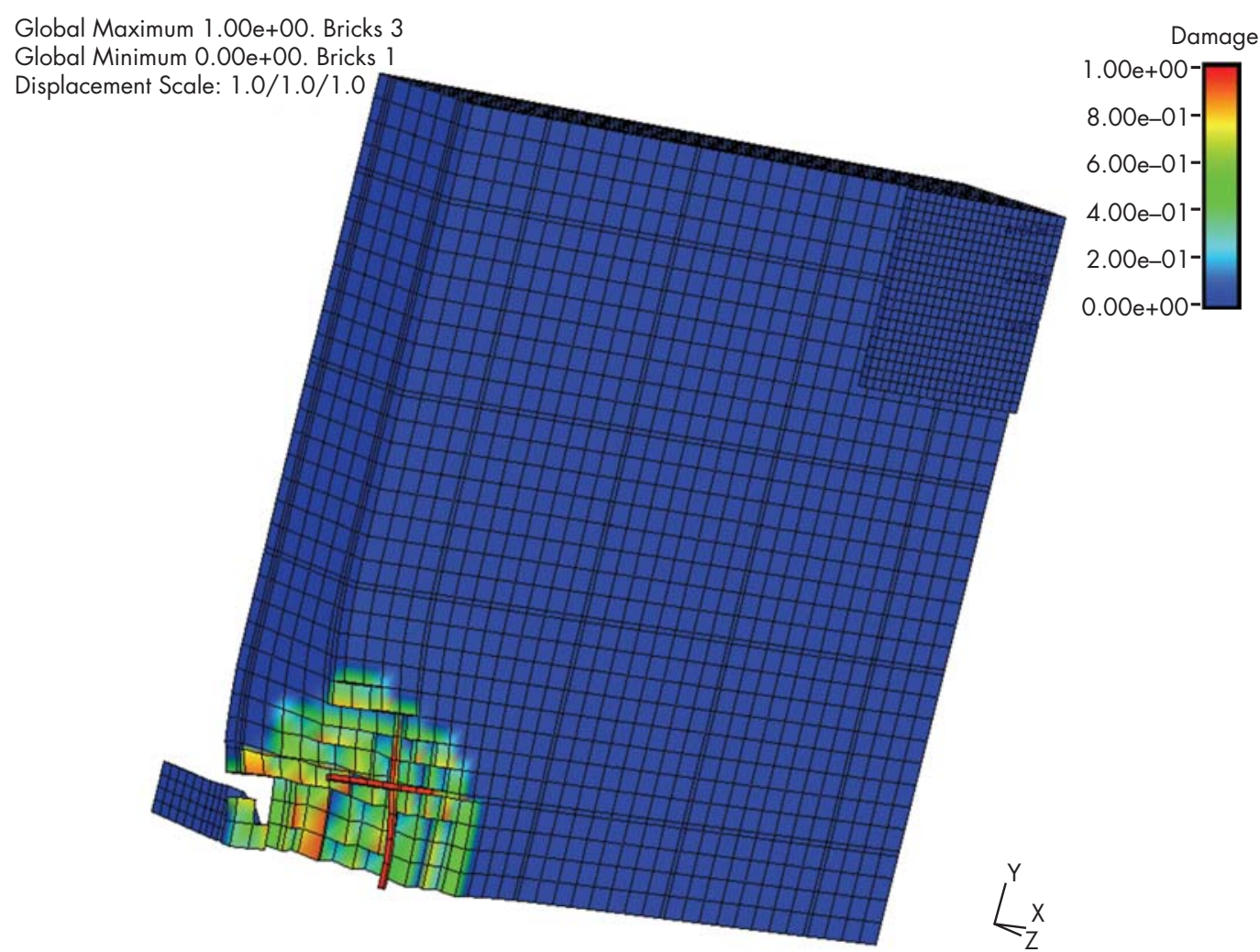

Figure 2. Penetration example with failed material made visible.

\section{FY2004 Accomplishments and Results}

In support of homeland security projects, we added a new capability to GRIZ that permits the user to define an evaluation metric that is a function of multiple response quantities. Our initial application was to help interpret the damage to concrete structures as calculated in DYNA3D. The concrete model in DYNA3D uses a damage parameter, $\mathrm{d}$. This parameter goes from 0 to 2, as the failure goes from initial yield to maximum failure to residual failure. This parameter should not be used by itself to determine structural integrity. The analyst needs to evaluate other indicators of damage, such as velocity, relative volume, or rebar strain, to get a complete picture of structural failure.

The command for the new result has four parameters: velocity direction, velocity magnitude cutoff value, relative volume cutoff value, and d cutoff value. For each element, a value of either 0 or 1 is assigned, depending on the composite of the average nodal velocity, relative volume, and the value of d. Figures 1 and 2 are examples of this feature applied to a simple, generic penetration problem. In Fig. 1, the elements interpreted as fully failed are colored red. In Fig. 2, these elements are made invisible, permitting visualization of the perforated structure.

A significant enhancement effort in Mili this past year was the implementation of a new surface class capability. This is an essential step needed for facilitating the viewing of boundary conditions and data from the analysis programs on surfaces.

Previously, the analysis programs used artificial (non-physical) shell elements and calculated a new material number for these elements, so that a "surface" could be written to the Mili database and then displayed using GRIZ. The new surface class eliminates such an ad hoc approach. The display of surfaces and results on a surface in GRIZ4s will be enhanced with a "surface manager," giving greater flexibility to view results such as boundary conditions and contact pressure information.

\section{FY2005 Proposed Work}

Due to the varying demands of user support and user-requested feature addition, our typical planning strategy is to identify a set of logical "next steps" for feature deployment. For next year these features include: completing the surface class capability deployment in GRIZ; addressing prioritized user-requested enhancements; and beginning implementation of Version 2.0 of the Mili I/O library, to support greater flexibility and emerging analysis capabilities like adaptive mesh refinement. 


\section{NIKE3D Enhancement and Support}

Figure 1. (a) Cyclic loading sequence for normal pressure on arched membrane. Pressure initially forces membrane downward to some given displacement and then reverses loading direction. (b) Pressure vs. normalized displacement for sequence in (a). The pressure loading begins positive but eventually becomes negative at "snap through." Pressure then becomes positive again as the membrane stiffens due to tension in the second of the four stages in (a). Pressure and sequence is then reversed.
LNL's NIKE3D, an implicit structuralmechanics finite-element code, is a first-class simulation tool. The objective of our work is to enhance, maintain, and support NIKE3D.

\section{Project Goals}

We are adding features to NIKE3D to accommodate the evolving needs of LLNL's engineering efforts. Maintenance includes fixing bugs and porting code to the various platforms available to analysts. User support includes recommending approaches and assisting analysts in debugging specific models.

\section{Relevance to LLNL Mission}

One of the important functions of engineering analysts is to ensure structural performance and integrity as program designs evolve. Our suite of codes

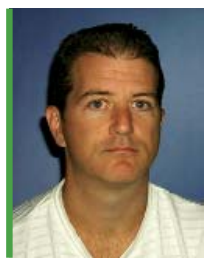

For more information contact Michael A. Puso (925) 422-8198, puso1@\|ln..gov

has a record of contributing to these activities over many decades, through sustained, responsive support of project analysts. NIKE3D, in particular, is an excellent code for handling difficult nonlinear static structural analysis problems.

\section{FY2004 Accomplishments and Results}

Continuation methods are nonlinear solution techniques used to solve analysis problems in the post-buckling regime of loading. The standard Newton-Raphson methods often fail in the post-buckling region when load control is applied.

Continuation methods modulate the applied loading to meet some target metric of deformation (such as arc-length) or force residual (minimum residual method). These techniques allow solution into the postbuckling regime.

The following improvements were made to NIKE3D:

Bug fixes. The arc-length methods pre-

(a)

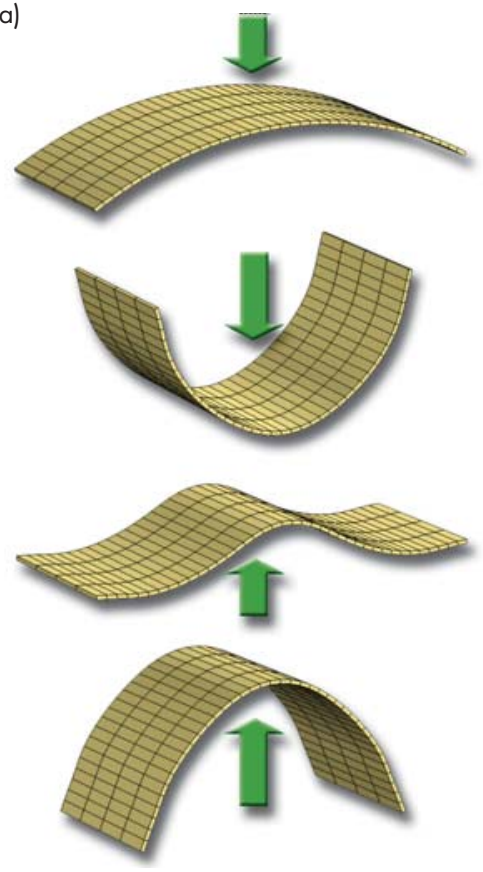
viously available in NIKE3D were a "work in progress" and consequently had multiple defects.

Pressure load control. The previous implementation provided support only for concentrated forces loadings, not pressures. (b)

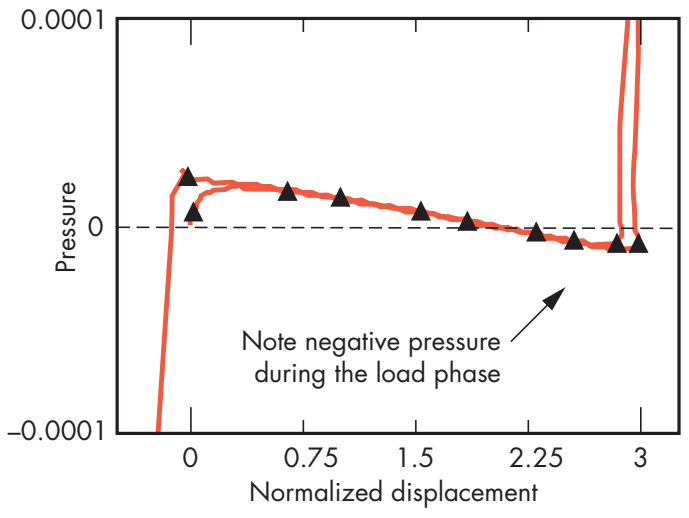


The latter is an important feature necessary for many post-buckling problems of shells.

Cyclic loading. Standard continuation implementations always require the load steps to advance the deformation. A cyclic loading capability was implemented so that the deformation can be applied cyclically, as seen in Fig. 1.

Minimum residual method. The minimum residual method was extended to work with our BFGS quasi-Newton nonlinear solver. The method often works better than the standard arc-length method.

A variety of additional continuum elements, using quadratic spatial discretization, were added to NIKE3D. These include 20- and 27-node hexahedra and 10-node tetrahedra. Available options include assumed strain versions with linear pressure distributions. The discretization error, measured as the energy norm of the error, converges linearly for linear element types (8-node hex and 4-node tet) and quadratically for the quadratic elements (Fig. 2). These elements can drastically reduce the overall degrees of freedom (DOFs) required to attain a desired amount of accuracy. For example, from Fig. 2, considering the data in the table: Point A corresponds to using 192 20-node brick elements, while Point B corresponds to 41,472 8-node brick elements, yet the discretization error is nearly the same. The CPU time for the equivalent accuracy is 5500 times greater with the 8 -node bricks.

\section{FY2005 Proposed Work}

Due to the varying demands of user support and user-requested feature addition, our typical planning strategy is to identify a set of logical "next steps" for feature deployment. For next year these include: the production version of mortar contact by generalizing algorithm data structures to make them compatible with the general features of NIKE3D; the implementation of a new anisotropic-damage material model for geological materials; and the incorporation of the capability to use a sharedmemory parallel direct equation solver on Linux computers.

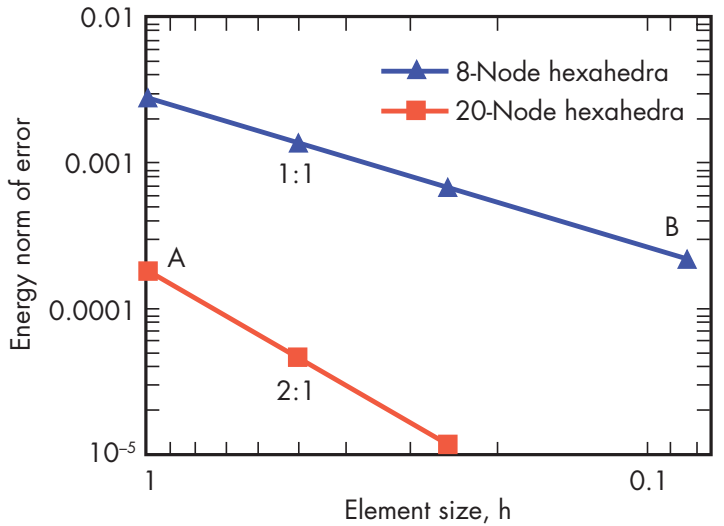

Figure 2. Discretization error for pressurized thick sphere with quadratic 20-node and linear 8-node hexahedra, vs. element size.

\begin{tabular}{|lccc|}
\hline & $\begin{array}{c}\text { Discreti- } \\
\text { zation } \\
\text { error }\end{array}$ & $\begin{array}{c}\text { Total } \\
\text { DOFs } \\
\text { in } \\
\text { model }\end{array}$ & $\begin{array}{c}\text { CPU time } \\
\text { for sparse, } \\
\text { direct } \\
\text { factorization }\end{array}$ \\
\hline $\begin{array}{l}\text { Point A } \\
\text { (20-node } \\
\text { bricks) }\end{array}$ & $\begin{array}{c}1.87 \\
\times\end{array}$ & 444 & $0.06 \mathrm{~s}$ \\
$10^{-4}$ & & \\
\hline $\begin{array}{l}\text { Point B } \\
\text { (8-node } \\
\text { bricks) }\end{array}$ & $\begin{array}{c}2.18 \\
\times\end{array}$ & 131,400 & $330 \mathrm{~s}$ \\
& $10^{-4}$ & & \\
\hline
\end{tabular}

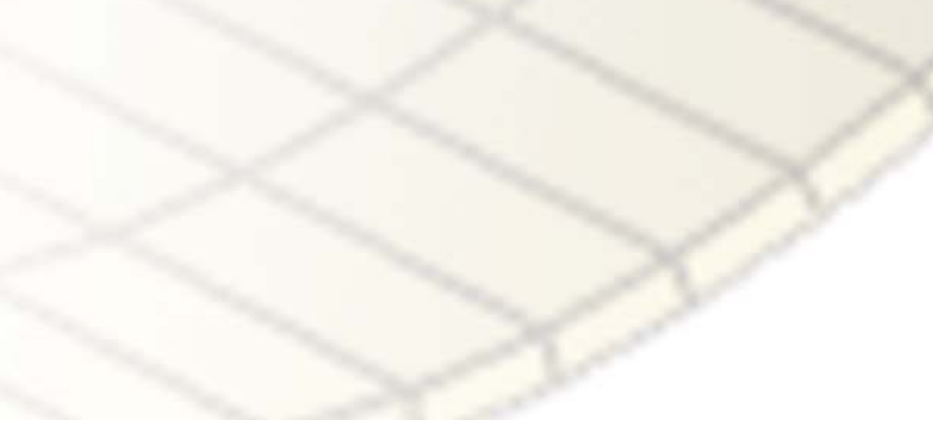




\section{E3D Modifications for Engineering Applications}

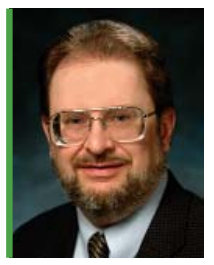

For more information contact David H. Chambers (925) 423-8893, chambers2@|ln..gov

3D is an elastic wave simulation code cused for prototyping and optimizing methods for acoustic imaging and detection. Typical applications include prototyping of NIF optics inspection systems (see Fig. 1); simulating acoustic imaging in tissue; acoustical tomography methods; buried waste imaging systems; and potting void detection. Future applications include acoustic inspection systems for NIF target characterization and investigation of methods for detecting flaws in layered media.

The original E3D code was written for seismic applications and later adapted for NDE applications. New users must typically edit the code to adapt it for their specific application. Over the years several versions of the code have been created with various undocumented features. This project consolidated these versions into a single code suite, optimized it for engineering applications, and constructed a user interface. The graphical user interface enables a larger number of engineers to use the code. In addition, a users guide was created for the NDE version, with examples.

\section{Project Goals}

The project was split into three parts: building a preprocessor, modifying the kernel, and creating postprocessing tools, with the preprocessor requiring the greatest amount of effort. Part of this effort entailed constructing models for transducers commonly used in NDE. These are required for direct comparison of simulated results with experiment.

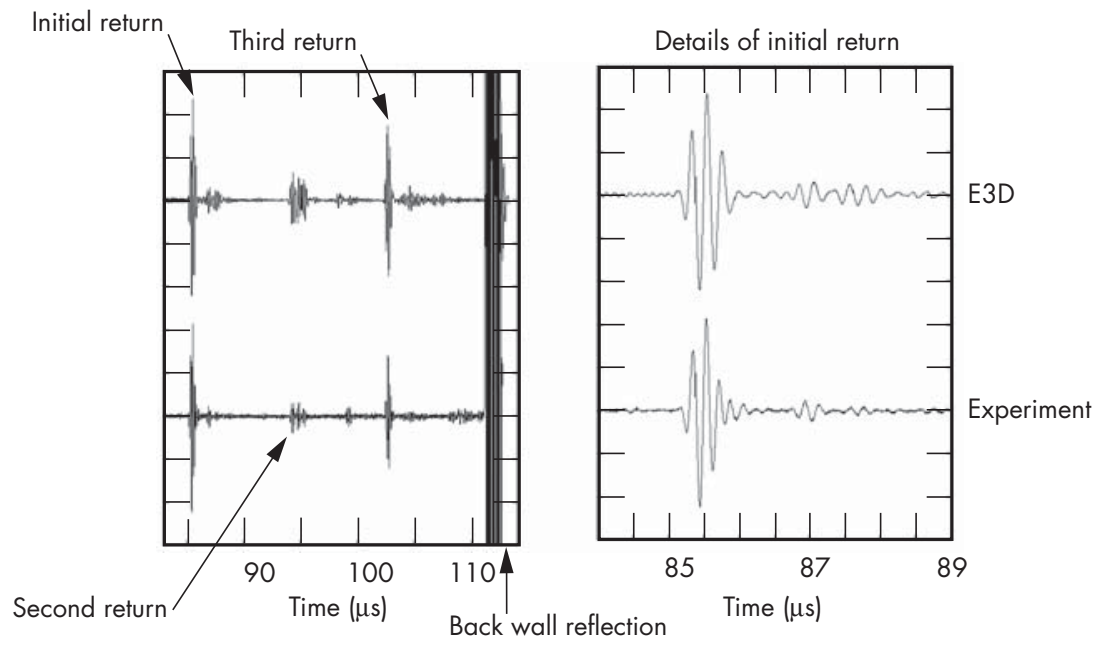

Figure 1. Acoustic reflections from surface pit in optical glass. Comparison between experiment and E3D simulation from reference article. 
We also tested the existing finite-element time-domain code, created for electromagnetic problems, on an elastic wave problem. These codes represent the future of timedomain modeling because of their high accuracy and geometric flexibility. The particular problem chosen was the normal vibrational modes for an aluminum sphere. The eigenvalues for the sphere provide a sensitive indicator of the accuracy of the method. Our results showed that the finiteelement code could calculate the first four eigenvalues to within $0.5 \%$. Figure 2 shows two constant-amplitude surfaces for the fourth vibrational mode.

\section{Relevance to LLNL Mission}

E3D is an existing code at LLNL. Consolidating the different versions already in use, adding a better interface, writing documentation, and testing with experiments has been a service to the users.

\section{FY2004 Accomplishments and Results}

We constructed a graphical user interface for setting up simulations. This allows a user to specify material properties for a variety of different objects in the computational domain. User-defined objects can also be imported using a specified input file format. A menu of pre-defined focused and unfocused transducers is used to specify both transmitting and receiving elements. These use generic models to translate input voltage to the appropriate acoustic source, or samples of acoustic fields to output voltages. After the user defines the simulation, the resulting computational domain can be displayed to verify the geometry.

The different versions of the core E3D program were consolidated and bundled in a format that could be installed on any platform with Fortran and $\mathrm{C}$ compilers.

To determine how well our existing differential form finite-element electromagnetic codes performed on elastic wave problems, we calculated the first four eigenvalues and eigenmodes of the aluminum sphere. The results agreed to within $0.5 \%$ of the theoretical values (Fig. 2 shows the fourth eigenmode). This shows the potential for a finite-element approach to elastic wave propagation. However, the present electromagnetic code can be applied to only a very limited range of elastic propagation problems. It lacks all the differential forms required for the full range of boundary conditions in elastic wave problems. We now know the specific forms that would create a fully general elastic wave code using finite elements.

\section{Related Reference}

Martin, L. P., D. H. Chambers, and G. H. Thomas, "Experimental and Simulated Ultrasonic Characterization of Complex Damage in Fused Silica," IEEE Trans. Ultrason., Ferroelect., Freq. Contr., 49 (2), pp. 255-265, 2002.

\section{FY2005 Proposed Work}

The pre- and postprocessors, new core program, and documentation are being installed on platforms in the NDE area. Additional modifications will be supported through general software maintenance or programmatic funding.

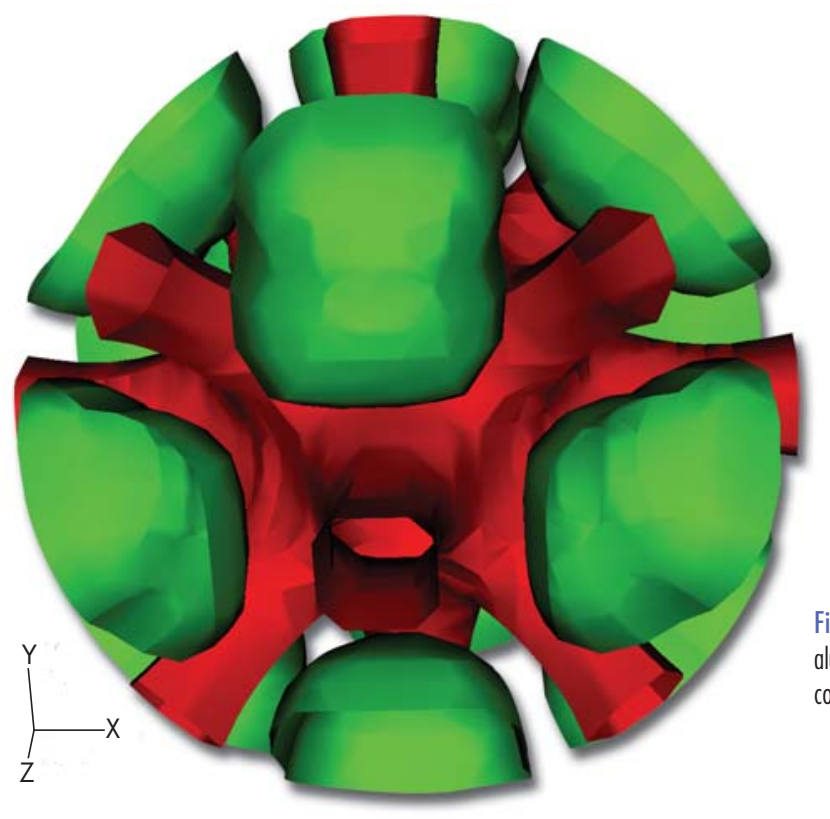

Figure 2. Constant amplitude surfaces for the fourth vibrational mode for an aluminum sphere. Calculation was performed by a high-order finite-element code originally for electromagnetic waves. 


\section{Engineering Visualization Theater}

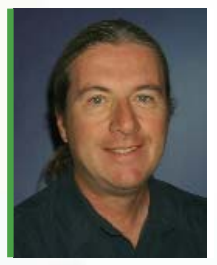

For more information contact Michael D. Loomis (925) 422-0364, loomis3@|lnl.gov

of media sources including computer display output, digital disc recorder, VCR, DVD, and a video network feed from LLTN. Presenters can easily patch a laptop computer into the system or use the resident hardware to display their material. The room provides seating for 10 to 15 people and provides an unclassified environment suitable for smallgroup collaborations, software demonstrations, or video presentations.

As a media production workshop, the facility also enables the creation of highquality visualizations to help engineers better communicate their ideas. The intent is to enhance good engineering with the best possible media tools and presentation techniques. High-end animation, compositing, and image processing software staffed by knowledgeable operators, provide the tools and expertise necessary to achieve this goal.

The hardware for this project includes two computers, a large screen display, a digital disk recorder, an S-VHS video recorder, a video monitor, a DVD player, surround sound speakers, an AV receiver, and a video switcher.

Software includes 3-D animation, compositing, image processing, video editing, DVD authoring, format conversion, and digital video compression. 


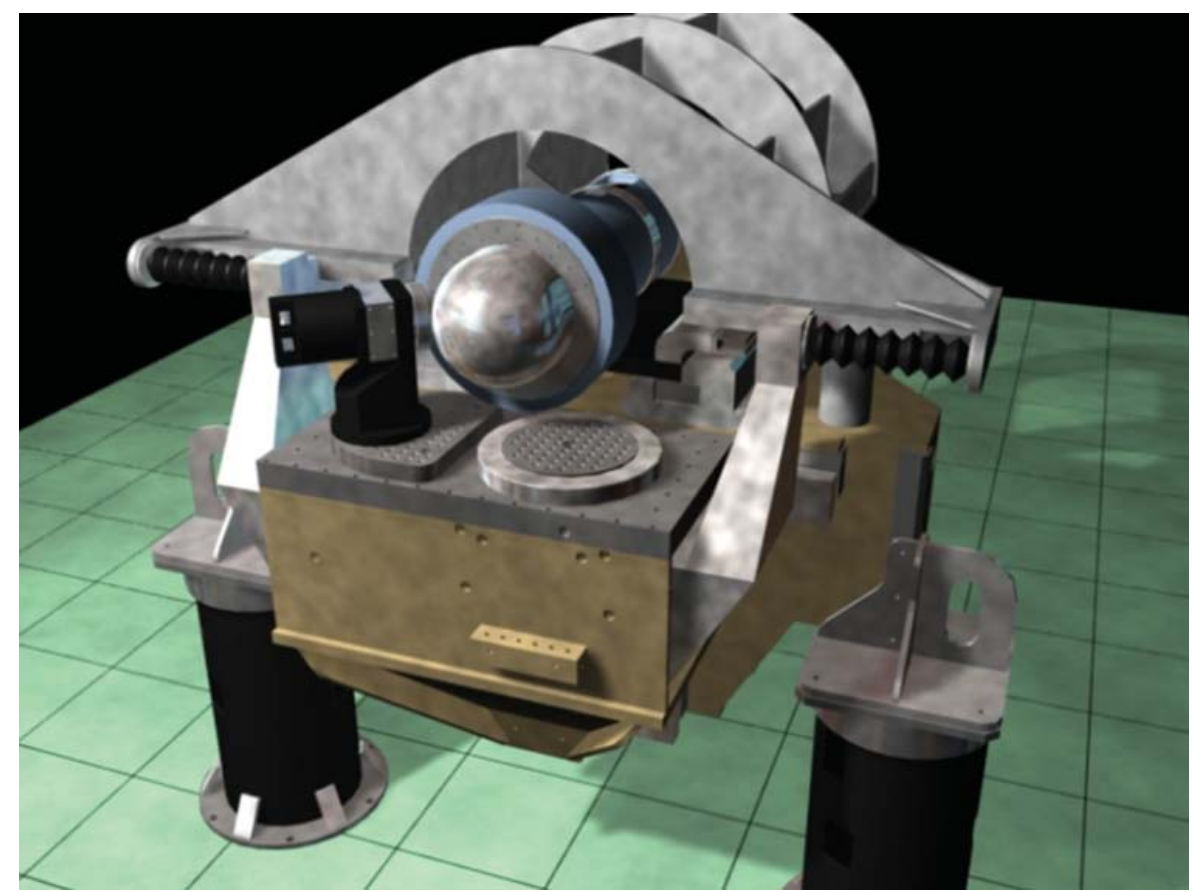

\section{Project Goals}

The primary goal of the EVT is to assist engineers in communicating their ideas. Specifically, the EVT aims to provide the ability to fuse engineering analysis and data with professional animation to clearly articulate complex concepts.

\section{Relevance to LLNL Mission}

The need for good communication is ubiquitous and on-going. Engineering needs to be able to articulate its ideas and results in a modern venue commensurate with its abilities and those of its customers.

\section{FY2004 Accomplishments and Results}

Production activity this year focused on two new movies. Like past EVT productions, it was desirable that the movies should fill a programmatic need; demonstrate a path for visualizing computational results by incorporating actual data; showcase various types of animation to illustrate the creative possibilities and techniques that are available for engineers to use in future animation productions; and generally demonstrate the EVT's capability.

The first movie was a visual aid to help communicate the basic concept of as-built modeling, as well as to provide a short case study illustrating the process of model characterization and simulation (see Fig. 1). EVT personnel worked closely with a team of engineers to create and execute a storyboard that would convey the significance of as-built analysis and present the results in an interesting way.

In the second project, the EVT provided a number of short animations to help visualize in three dimensions how tool motion errors can introduce surface anomalies in precision machining processes (see Fig. 2). Computer animation was particularly effective in this case because of its ability to illustrate 3-D concepts directly. In each case, the result
Figure 2. Illustration from short animation to help visualize in three dimensions how tool motion errors can introduce surface anomalies in precision machining processes.

was a movie that brought engineering ideas to life in a form that was both instructive and visually compelling.

These and other animations were previewed for several key engineering personnel on the large video screen in the EVT facility. By combining creative media production with the display capabilities inherent in the assembled hardware, we believe that the presentation demonstrates the potential for using the EVT as a tool to effectively communicate engineering concepts.

\section{FY2005 Proposed Work}

The EVT intends to provide its services on an on-going basis. 


\section{Enhanced Fluid Dynamics Modeling Capability}

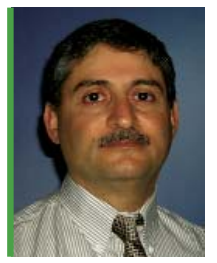

For more information contact Kambiz Salari (925) 424-4625, salaril@|ln..gov

his is a brief effort to demonstrate and benchmark the computational fluid dynamics modeling capability of STAR-CD.

\section{Project Goals}

To evaluate the numerical discretization behavior, solver convergence, grid generation capabilities, and parallel implementation of STAR-CD, three benchmark cases were selected: steady, fully-developed flow through a curved, circular pipe; pulsatile, fully-developed flow through a curved pipe; and thermal convection within a pipe. The first two benchmark problems present complex, 3-D flows, and the third exercises the heat transfer modeling capability of STARCD. Additionally, an application problem of flow through a simplified aneurysm geometry was computed.

\section{Relevance to LLNL Mission}

This effort is aligned with the LLNL science and technology goals because it provides a general fluid dynamics modeling capability that can be used by many programs, such as those in Engineering, NAI, DNT, and Computations.

\section{FY2004 Accomplishments and Results}

The geometry for the first benchmark problem is a $180^{\circ}$ curved pipe with a radius ratio of $\delta=a / R=1 / 20$, where $a$ is the pipe radius and $R$ is the radius of pipe curvature (Fig. 1). The simulations are run on three grids $(23185,155100$, and 1242808 elements) to test for grid convergence. The computational domains are generated with STAR-CD's semiautomated meshing capability, which significantly reduces the effort. For the coarse mesh, the velocity residuals decrease to $10^{-5}$ and remain unchanged with further iterations, while for the medium and fine meshes, the velocity residuals decrease to $10^{-6}$ and remain of that order for the simulation.

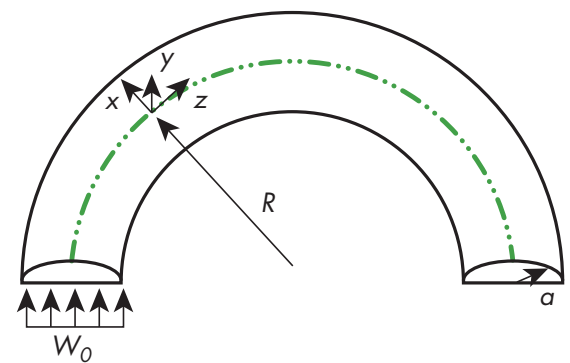

Figure 1. Geometry of the $180^{\circ}$ curved pipe used in the first benchmark case. $W_{0}$ is the mean inlet velocity. 
The geometry for the second benchmark case is a $90^{\circ}$ curved pipe with a radius ratio of $\delta=a / R=1 / 6$. The selection of the necessary mesh size was guided by the simulations conducted in the first benchmark case. To determine the sensitivity of the computed solution to the location of the inlet and outlet boundary conditions, three different boundary condition locations are chosen. The computed results do show some sensitivity. The results of this benchmark case demonstrate that it may be necessary to extend the locations of the inlet and outlet boundary conditions away from the region of interest to properly capture a pulsatile flow field.

The third benchmark problem is that of convective heat transfer in a circular pipe (Fig. 2). To determine the sensitivity of the solution to the grid topology, grid refinement level, and pipe length, six different grids ranging in size from $10^{4}$ to $10^{6}$ elements are used in calculating the steady state solution of the momentum and energy equations.

Having benchmarked STAR-CD against internal flow problems, an application problem of flow through two generic basilar aneurysms was selected for simulation. The computational domains of the aneurysms and surrounding arteries are shown in Fig. 3. A steady, parabolic velocity profile is specified at the inlet to the basilar artery for both cases. The wall shear stress is relatively small throughout the entire aneurysm dome. A more thorough calculation and examination of the flow field for the second arterial geometry were also conducted in a separate project.

The strengths of STAR-CD are semiautomated mesh generation, availability of multiple numerical schemes, a variety of solvers, flexible boundary condition specification, local mesh refinement capability, and quick simulation setup. The simulations were run on LLNL's "Bear" Linux cluster using up to four processors for the first benchmark case. The solvers perform consistently over a range of grid sizes and the time-dependent solution for the second benchmark case is well behaved even for large time step increments.

\section{Related Referncess}

1. Adler, M., Z. angew. Math. Mech. 14, p. 257, 1935. 2. Agrawal, Y., L. Talbot, and K. Gong, Journal of Fluid Mechanics, 85, p. 497, 1978.

3. Collins, W. M., and S. C. R. Dennis, Quart. J. Mech. Appl. Math., 28, p. 133, 1975.

4. Incropera, F. P., and D. P. DeWitt, Fundamentals of Heat and Mass Transfer, Wiley, New York, New York, 1990.

5. Rindt, C. C. M., A. A. van Steenhoven, J. D. Janssen, and G. Vossers, Journal of Fluid Mechanics, 226, p. 445, 1991.

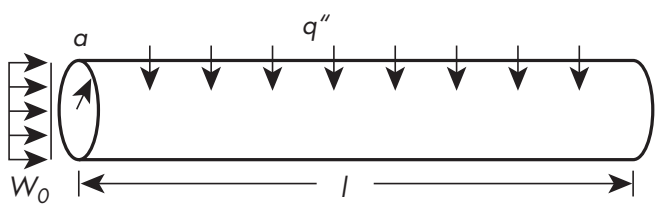

Figure 2. Computational setup for convective heat transfer in a circular pipe.
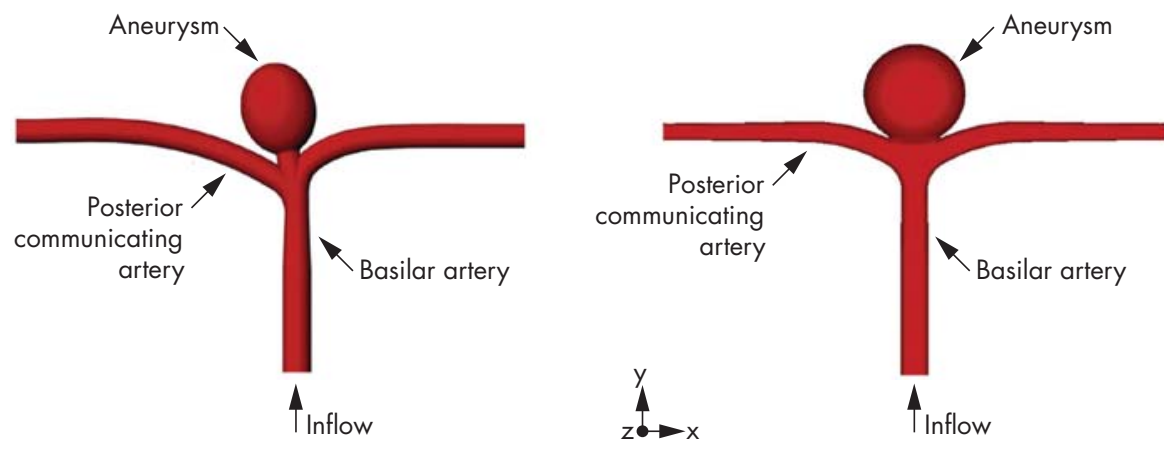

Figure 3. Computational domains of the two generic basilar aneurysms. 


\section{Enhanced Fluid Dynamics Modeling Capability for Endovascular Flows}

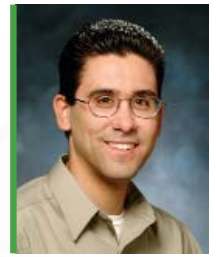

For more information contact Jason Ortega (925) 423-3824, ortega17@\|nl.gov

these changes contribute to the progression of a disease. This may result in not only a better understanding for the causes of cardiovascular disease, but also improved treatment techniques.

CFD can also be used as a testbed for advanced interventional devices. Before performing animal-based studies, interventional devices, such as drug-releasing stents or aneurysm-filling foams, can be simulated within endovascular flowfields to determine the device's performance capabilities. Questions as to whether or not the released drugs accumulate in the target regions, or how the aneurysm-filling foams impact the flowfield within parent arteries can be answered early in the process and properly addressed.

\section{Project Goals}

To lay the groundwork for simulations of actual endovascular systems, the goal of this project is to perform validation simulations of the flow within a generic, terminal aneurysm on a basilar artery with a CFD software package, STAR-CD. The results of the aneurysm simulations will be compared with experimental data obtained from the Fluid Mechanics Laboratory at the University of California, Berkeley.

\section{Relevance to LLNL Mission}

The technology to accurately predict the complex, unsteady, 3-D flow through the endovascular system could have an important homeland security application in simulating the flow through the human respiratory system and determining the transport and deposition of airborne pathogens.

Figure 1. Angiogram of a basilar aneurysm within the brain. 


\section{FY2004 Accomplishments and Results}

The basilar artery is located within the brain and is a common location for aneurysm formation. An example of a terminal basilar aneurysm is shown in the angiogram in Fig. 1. The diameter of the basilar artery $(3.23 \mathrm{~mm})$ and the diameter of the aneurysm $(11.7 \mathrm{~mm})$ used in the STAR-CD simulations are average physiological values, and are shown in Fig. 2. For the simulations with STAR-CD, the boundary condition at the inlet to the basilar artery is a steady flowrate of 1.85 $\mathrm{cm}^{3} / \mathrm{s}$, which is approximately equal to the physiological value.

The unsteady, incompressible NavierStokes equations for blood flow with a constant viscosity are solved with
STAR-CD using a second-order spatial discretization scheme. Information about the flowfield can be obtained by examining isosurfaces of the helicity (the inner product of the velocity and vorticity vector fields), which is a useful quantity for identifying regions of swirling flow.

Figure 3 shows isosurfaces of positive and negative helicity in the basilar bifurcation region. The distribution of these surfaces indicates that counterrotating regions of flow form as the flow turns from the basilar artery into the posterior communicating arteries. The velocity field at a center cross-section of the aneurysm was also studied: a jet forms at the neck of the aneurysm and extends upwards, where it impinges directly upon the tip of the aneurysm. This is an important result since aneurysms often rupture at this location. The results of these simulations demonstrate that STAR-CD can be a valuable tool for obtaining detailed information about the physics of flows within endovascular systems.

\section{Related References}

1. Parlea, L., R. Fahrig, D. W. Holdsworth, and S. P. Lownie, "An Analysis of the Geometry of Saccular Intracranial Aneurysms," American Journal of Neuroradiology, 20, pp. 1079-1089, 2003.

2. Stehbens, W. E., "Aneurysms and Anatomical Variation of Cerebral Arteries," Archives of Pathology, 75, pp. 57-76, 1963.
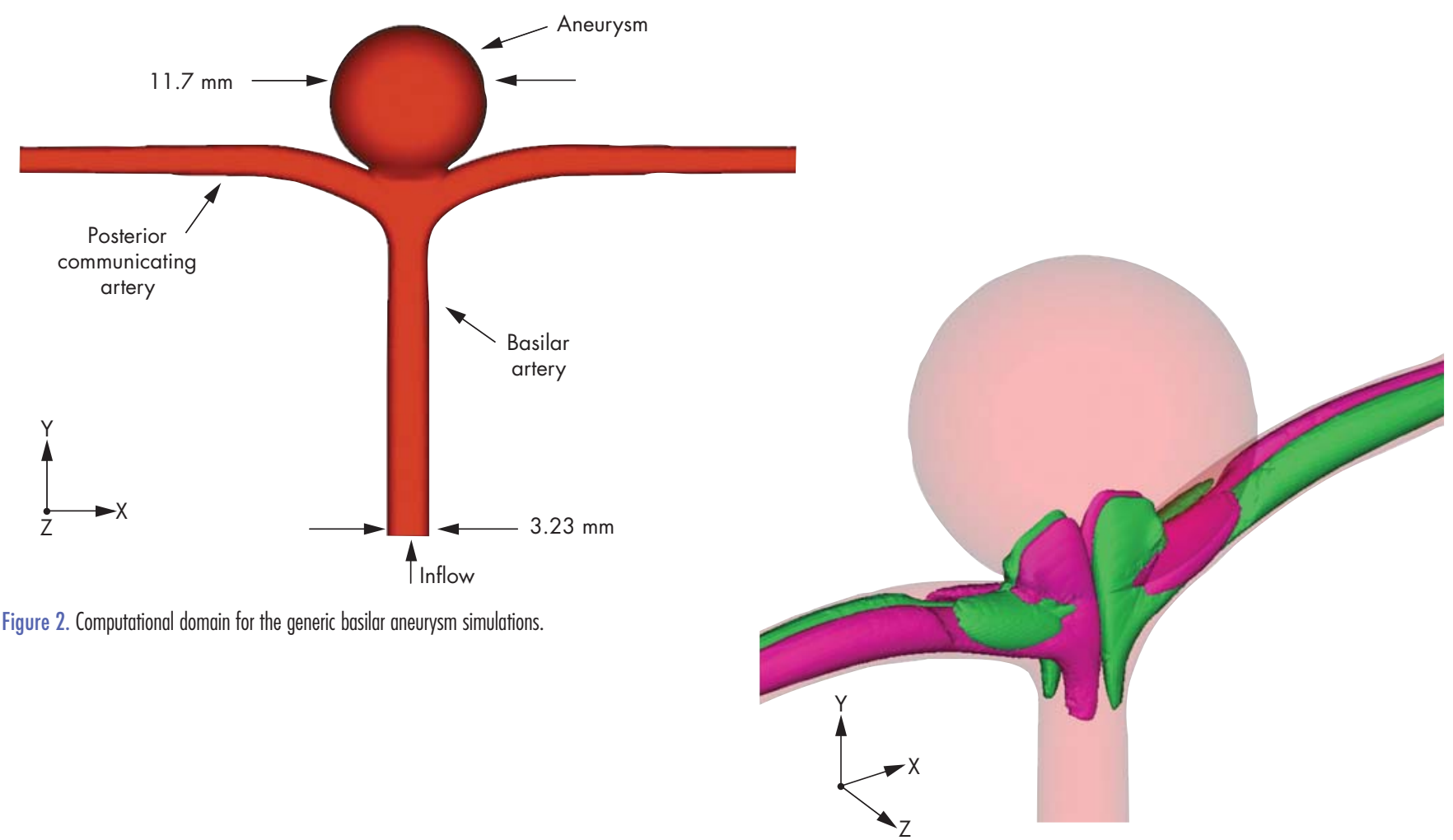

Figure 3. Positive (green) and negative (magenta) isosurfaces of helicity in the basilar bifurcation region. 


\section{Modeling Initiation in Exploding Bridgewire Detonators}

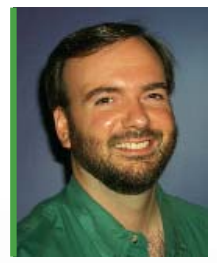

For more information contact Constantine A. Hrousis (925) 423-4744, hrousis1@|ln..gov

\section{Project Goals}

The deliverables of this project include: here is great interest in the dynamics
of exploding bridgewire (EBW) performance and its role in the process of initiation in EBW detonators. In particular, we need to better understand the mechanism by which electrical energy stored in a fireset transforms into initiating energy within a high explosive, and how this mechanism is altered by changing materials and geometry. This project uses LLNL's magnetohydrodynamic (MHD) code CALE to model the explosion of EBWs when placed in a circuit with a fireset.

The models have been generalized from 1-D to 2-D, with the capability of including aging effects, such as the growth of intermetallic compounds. sufficiently accurate 2-D models for pure metal EBWs surrounded by high explosive; models that predict performance of aged systems; and experiments to validate the MHD models in CALE at low energies, and the associated analyses needed for model validation. The final product is a simulation capability for EBW and slapper initiation in arbitrary configurations, an assessment tool for detonator reliability.

\section{Relevance to LLNL Mission} because the aging of soldered gold EBW detonators is not sufficiently understood. This simulation capability will enhance LLNL's predictive toolset, which is critical to LLNL's stockpile stewardship mission.
This problem is of particular interest (a)

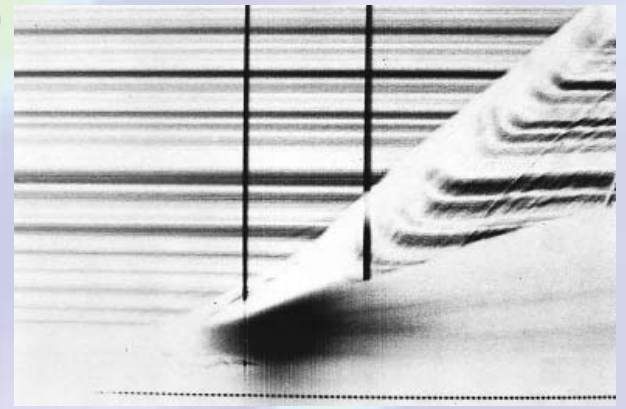

(b)

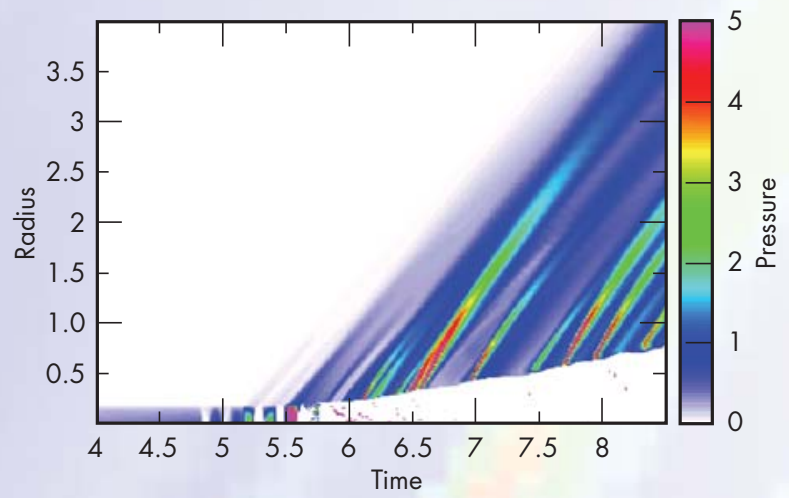

Figure 1. (a) Experimental streak image of a gold wire exploding in water. Time increases to right; the radius increases upward. Note wire expansion, followed by the material expansion (lower sloped curve) and the shock wave sent into the surrounding medium (upper sloped curve). (b) 1-D simulation of the underwater EBW experiment, presented as a streak image. Colors depict pressure levels in the wire and water. 
(a)

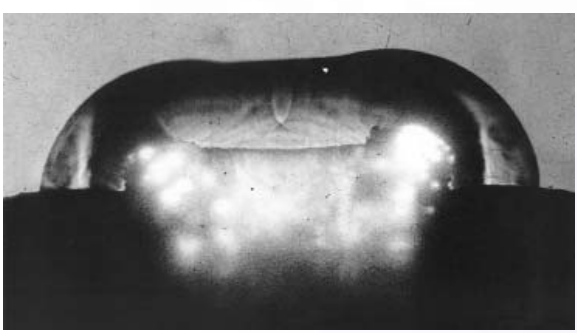

(b)

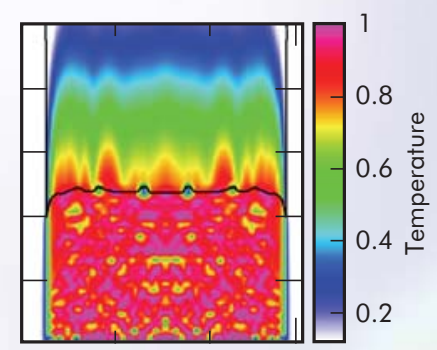

Figure 2. (a) Experimental frame image (side view) of a gold wire luminescing as it explodes. (b) Preliminary 2-D longitudinal simulation of the underwater EBW experiment. Colors show material temperature levels.

\section{FY2004 Accomplishments and Results}

One-dimensional models of wire burst were compared with recent and historical closed-form calculations, as well as underwater wire burst experiments and full detonator test fire experiments. The 1-D model predicts most metrics of interest (such as time of burst and burst current) with reasonable accuracy, but is still in need of improvement in predicting EBW pressure output. Figure 1 demonstrates qualitative agreement between experiment and 1-D simulation streak images.

Also, 2-D models were created to better understand both longitudinal and axial aspects of EBW performance. Figure 2 shows comparison between a longitudinal view of an exploding gold bridgewire, and a 2-D CALE simulation. The incorporation of a preliminary material model for goldindide is a recent accomplishment, due in part to experimental studies completed to find this material's coefficient of thermal expansion. This property is needed for estimation of the Gruneisen gamma, a parameter that influences both shock and MHD aspects of the material's response.

\section{Related References}

1. Chace, W. G., and H. K. Moore, Exploding Wires, Plenum Press, Inc., New York, New York, 1959.

2. Maninger, R. C., "Predicting Time-of-Burst of Exploding Bridgewires from Thermodynamic and Electrical Properties," 4th Symposium on Engineering Aspects of Magnetohydrodynamics, April 1963.

\section{FY2005 Proposed Work}

We have plans for model validation through underwater burst experiments on specially made EBWs of various materials. In particular, the material gold-indide requires further experimental study to be successfully integrated into our EBW models. The models will be further generalized to make predictive statements about initiation in PETN, a high explosive commonly used with EBWs, in effect, simulating the crux of detonator function. 


\section{Modeling Production Plant Forming Processes}

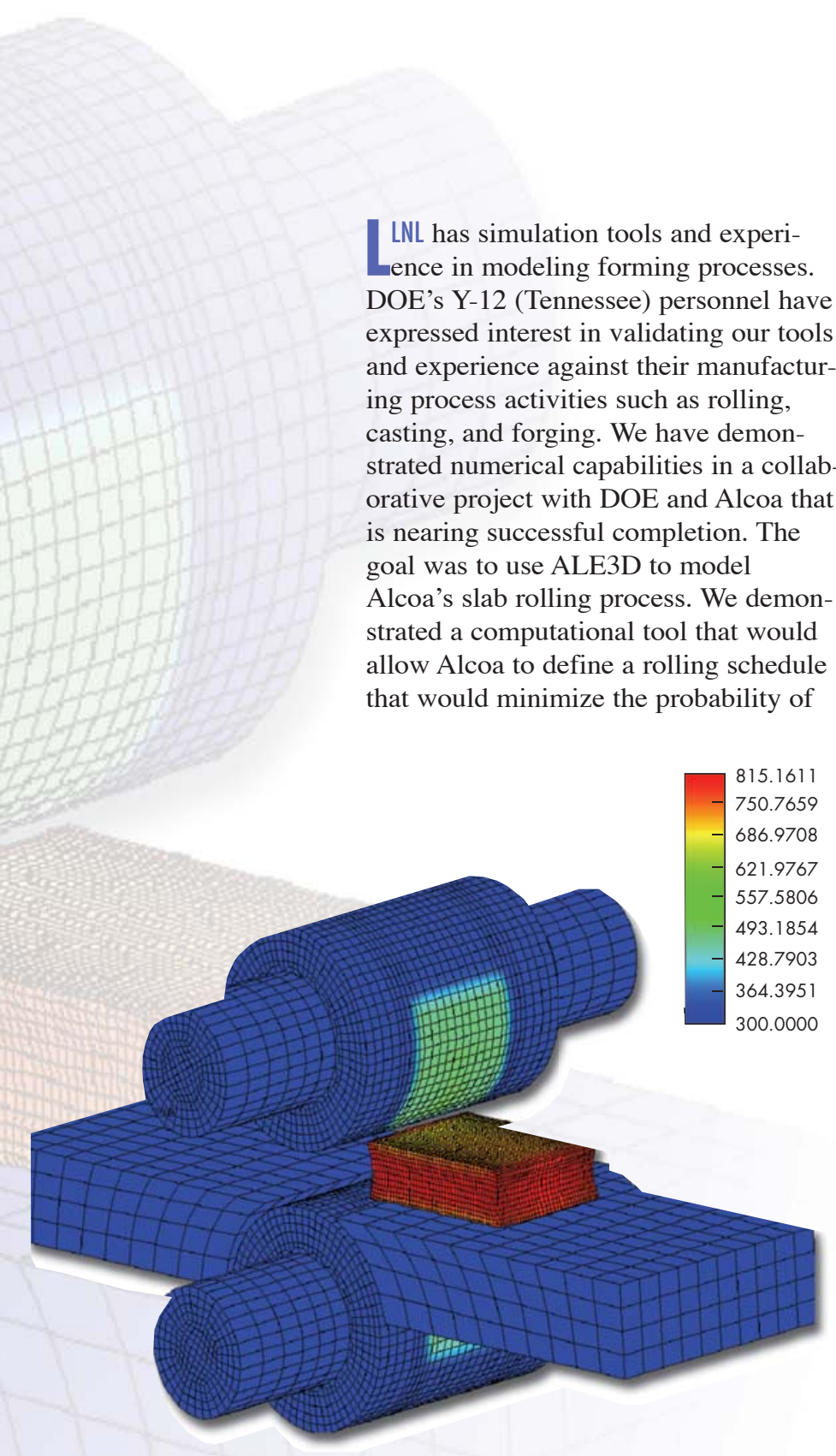

Figure 1. Illustration of a finite-element model representing rolls, slab, and supports. Thermal contours are shown.

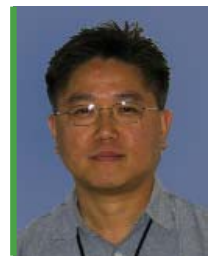

For more information contact Moon Rhee (925) 424-4990, rhee1@\|nl.gov

ingot fracture, thus reducing waste and energy consumption. We intend this to lead to long-term collaboration with Y12 , and perhaps involvement with other components of the weapons production complex. Using simulations to aid in building forming processes can decrease time to production; reduce forming trials and associated expenses; and guide the creation of products with greater uniformity and less scrap.

\section{Project Goals}

The immediate objective of the proposed work is to use our numerical capabilities for rolling process validations and to foster a close collaboration with Y-12. We propose to validate our ability to model rolling experiments and assess our ability to address other forming processes at Y-12.

The experience gained here would translate to other Laboratory projects.

\section{Relevance to LLNL Mission}

Modeling forming processes and material response is a significant responsibility of LLNL's Engineering Directorate. This effort will contribute to Engineering's core competency in numerical modeling and material response. The capability demonstrated in this project would be supportive of other forming projects where geometric fidelity and control of material properties are critical. A successful effort will also contribute programmatically to DNT objectives. 


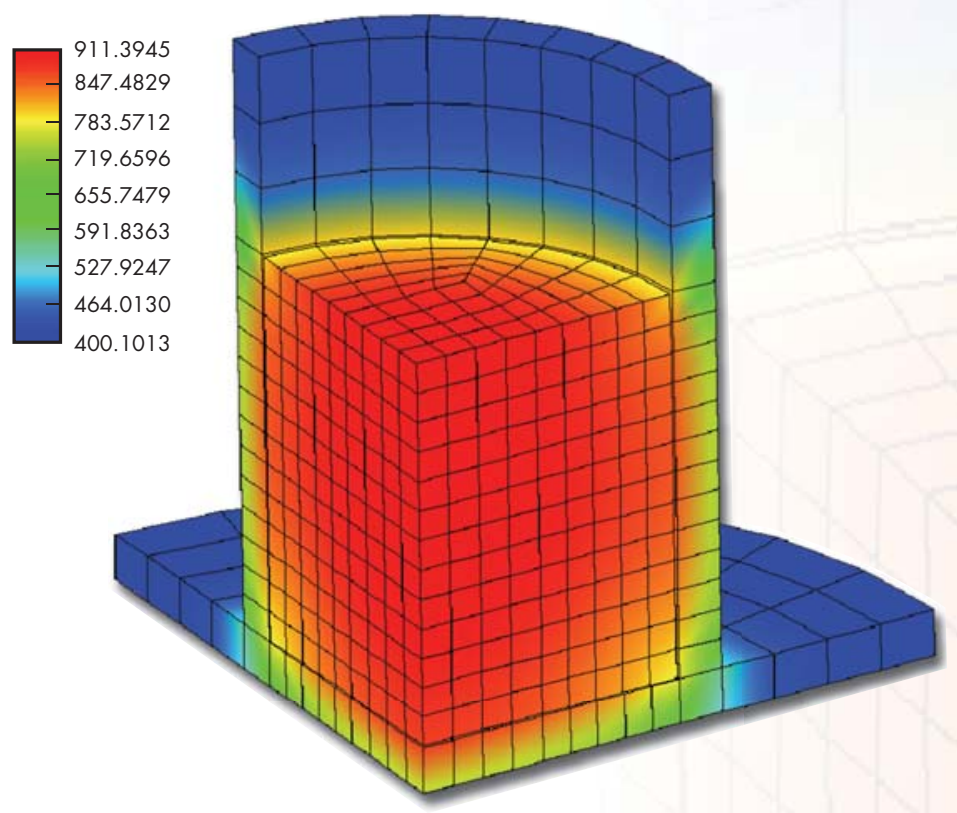

Figure 2. Illustration of a casting simulation showing thermal contours.

\section{FY2004 Accomplishments and Results}

LLNL personnel visited Y-12 to accomplish the following: familiarize ourselves with the suite of Y-12 forming issues (see Figs. 1 and 2), including advanced concepts, and identify areas where improved modeling might provide a significant benefit; and assess existing material properties data and identify approaches for filling gaps in knowledge.

The technical interchange at Y-12 was successful, yielding: information relevant to Y-12 modeling and forming processes; an agreement to collaborate on FY2005 technology-base activities; a path forward to produce a joint ADAPT proposal focused on understanding deformation forming processes.

We have performed a preliminary survey of high-temperature material properties for use in simulations, and have begun a series of calculations to evaluate the capability of ALE3D to model casting (Fig. 2).

An ADAPT proposal will be produced by the end of FY2004. The exit plan of this project is transition to an ADAPTfunded project in the future.

\section{FY2005 Proposed Work}

We will collaborate with $\mathrm{Y}-12$ personnel to determine a rolling event and other forming data that can be used as validation data for our codes and techniques. We will attempt to predict microstructural evolution as well as geometric data. Knowledge of material properties relevant to forming regimes will be exchanged. Rolling validation calculations will be performed against rolling data from Y-12.

In our validation of ALE3D for reproducing rolling data, we are considering geometric fidelity and the evolution of microstructure.

Where appropriate we will perform code validation calculations, such as evaluating casting modeling codes against Y-12 specific data. 


\section{Modeling Rarefied Gas Flows with Direct Simulation Monte Carlo}

A nalysis of fluid transport between the continuum and molecular regimes is critical to characterizing and optimizing instruments and vehicles that operate in these conditions. For example, gas transport in microfluidic devices exhibits phenomena that are not present in macroscopic applications. Effects such as rarefaction, thermal creep, and slip boundaries play an important role at microscales and nanoscales and cannot be accurately modeled by continuum solvers due to the molecular nature of the flow.

The degree of noncontinuum behavior is determined by the Knudsen number, the ratio of the mean free path to a characteristic length scale of the flow. Figure 1 depicts the different Knudsen regimes and representative applications. In addition to microfluidic applications, Knudsen number effects are significant in rarefied gas environments and across large gradients such as strong shocks. We intend to implement a direct simulation Monte Carlo (DSMC)

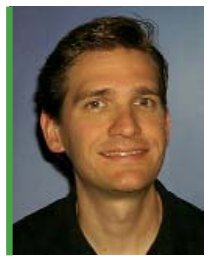

For more information contact Todd Weisgraber (925) 423-6349, weisgraber2@||ln..gov

capability for subcontinuum fluid flows.

DSMC is a particle-based approach to solving the Boltzmann transport equation. Because the simulated particles are representative of the larger number of gas molecules in the fluid, DSMC is more efficient than purely atomistic simulations like molecular dynamics. Though the original method is applicable to dilute gases, DSMC can be generalized for dense gases with an arbitrary equation of state.

This particle model can be implemented into a computational tool to analyze gas flows in microscale geometries and in larger systems at the dilute limit (high Knudsen number) where the continuum hypothesis is not valid. DSMC has been successfully applied to rarefied and hypersonic flows, but it is also relevant to particle beam focusing, micro-propulsion systems, inertial confinement fusion, and fabrication processes such as vapor deposition and ion etching.
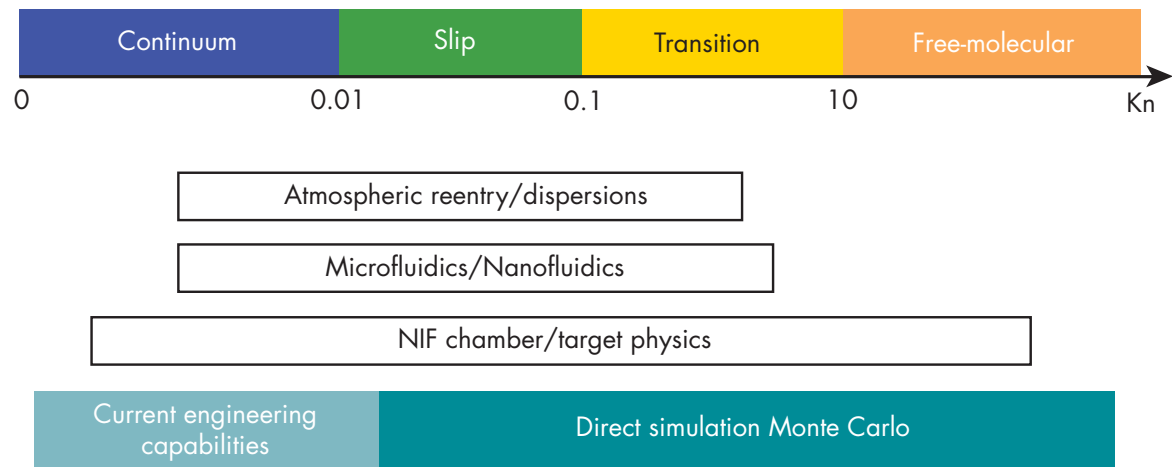

Figure 1. Knudsen number regimes. 


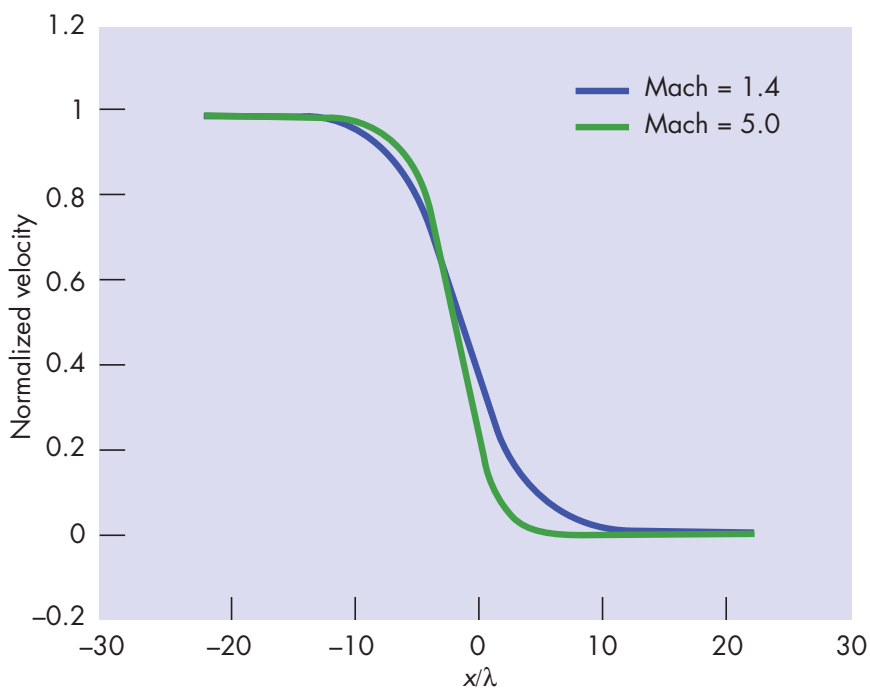

Figure 2. Velocity shock profile for argon gas.

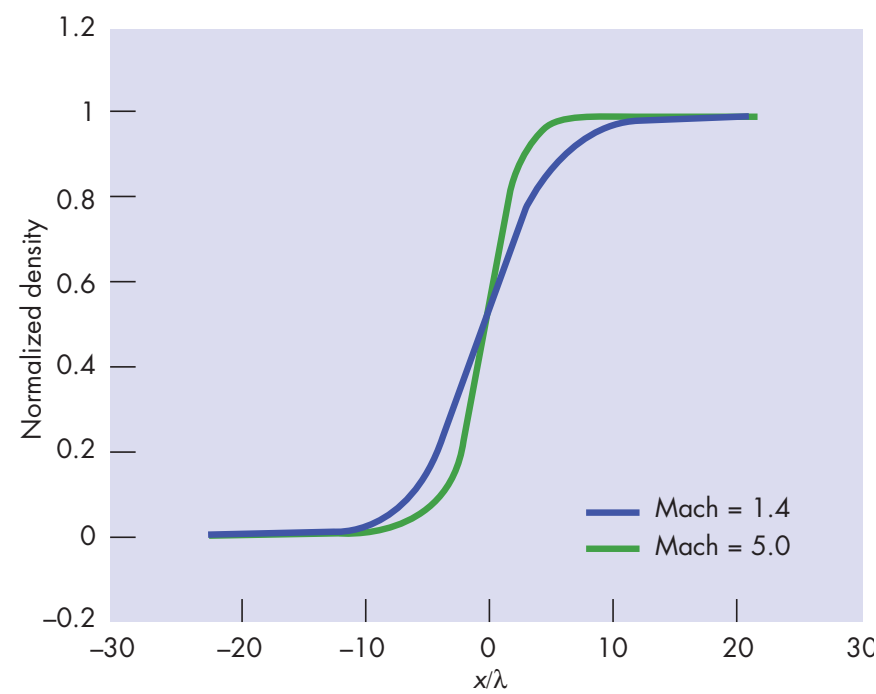

Figure 3. Density shock profile for argon gas.

\section{Project Goals}

We will construct a plan for noncontinuum fluid modeling using DSMC methods in the public domain. We will implement these flow solvers and demonstrate this approach for gas flows at both small (microfluidics) and large (hypersonic flight) length scales.

\section{Relevance to LLNL Mission}

The implementation of a DSMC algorithm for gas flows will provide LLNL with a new capability relevant to fusion energy and sensors for homeland security. From a scientific perspective, DSMC is an effective tool to investigate nanoscale and noncontinuum physics. A fundamental understanding of phenomena in these regimes will motivate the development of novel devices and systems.

\section{FY2004 Accomplishments and Results}

We surveyed potential applications and proven noncontinuum methods. Some of the identified applications for DSMC are indicated in Fig. 1. We also identified published and public domain codes to establish a baseline for features to incorporate into our code. As a basic demonstration of DSMC, we implemented a solver for flow across a stationary shock. Figures 2 and 3 show the shock structure for argon with upstream Mach numbers of 1.4 and 5 . The width of the shock is expressed relative to the mean free path.

\section{Related References}

1. Alexander, F. J., A. L. Garcia, and B. J. Alder, "A Consistent Boltzmann Algorithm," Phys. Rev. Lett., 74, pp. 5212-15, 1995.

2. Bird, G. A., Molecular Gas Dynamics and the Direct Simulation of Gas Flows, Oxford, 1994.

3. Hudson, M. L., and T. J. Bartel, "Direct Simulation Monte Carlo Computation of Reactor-Feature Scale Flows," J. Vac. Sci. Technol., A 15, (3), pp. 559-63, 1997.

4. Oran, E. S., C. K. Oh, and B. Z. Cybyk, "Direct Simulation Monte Carlo: Recent Advances and Applications," Ann. Rev. Fluid Mech., 30, pp. 403-41, 1998.

\section{FY2005 Proposed Work}

We have proposed to implement and validate a full 3-D DSMC algorithm based on proven methods. We will also extend the capability of the algorithm to dense gases based on a modified Boltzmann approach. Finally, we will provide a demonstration of the code by simulating flow in a microchannel and a jet expansion downstream of a focusing nozzle. As part of our exit strategy, we will explore potential methodologies to couple the particle simulations with continuum solvers and thus provide an interface with other LLNL capabilities. 


\section{Sensitivity Analysis in Modeling the Fate and Transport of Chemical and Biological Agents}

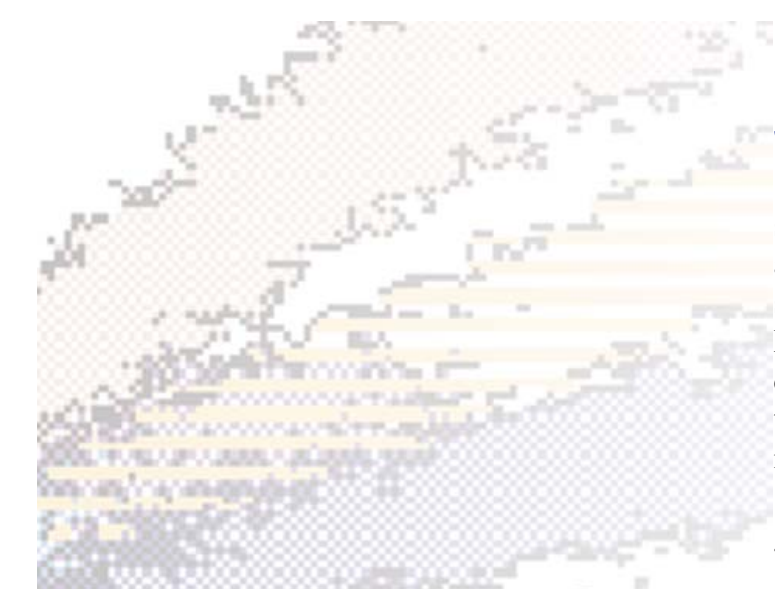

W e are focusing on a problem of importance to national security: the dispersion of chemical or biological agents in the atmosphere, in conjunction with the large, sophisticated computational models used by NARAC. The project is intended as a logical first step for exploring the reductionto-practice of sensitivity theory to national/homeland security applications.

In the first year, we focused on investigating the use of first-order sensitivity

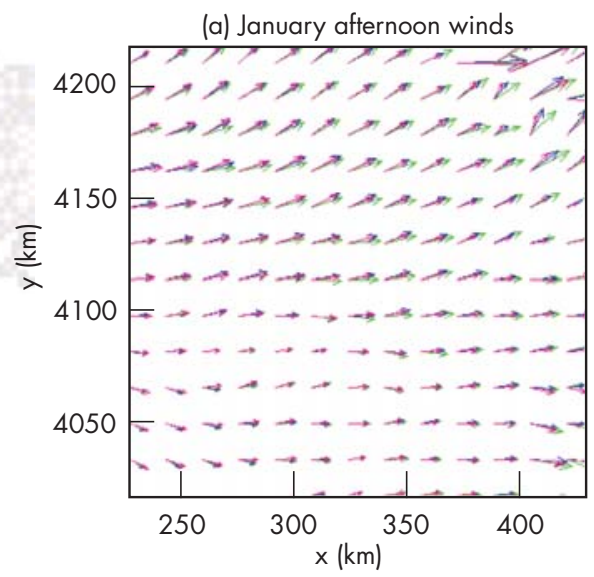

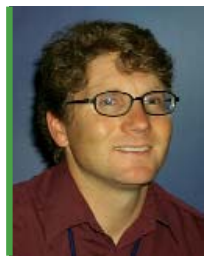

For more information contact Lee Greer Glascoe (925) 423-2922, glascoe1@|ln..gov

analysis to examine the effect of small perturbations in input parameters on the output of the problem. In the second year we shifted the focus to sensitivity and uncertainty analysis of multidimensional, multivariate, time-dependent wind fields, because these play a dominant role in the uncertainty of atmospheric dispersion prediction. We focused on building a tool from existing methods to characterize and reduce a large multivariate meteorological time series.

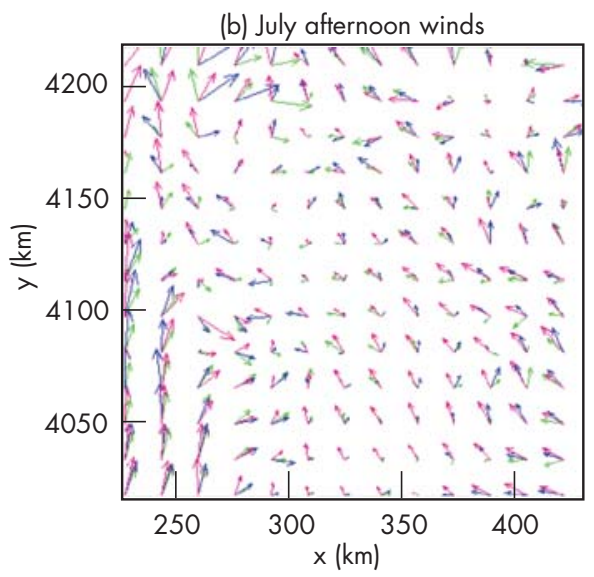

Figure 1. Time-varying wind fields on (a) January and (b) July afternoons at 406-m altitude ( 3 p. m. in magenta, 4 p. m. in blue, 5 p. m. in green). Domain shown is $\sim 200 \mathrm{~km}$ on a side; center magenta wind vector in January is $\sim 5 \mathrm{~m} / \mathrm{s}$.

(a) January release

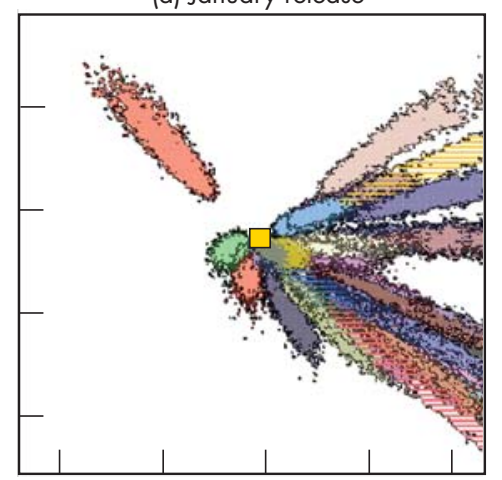

(b) July release

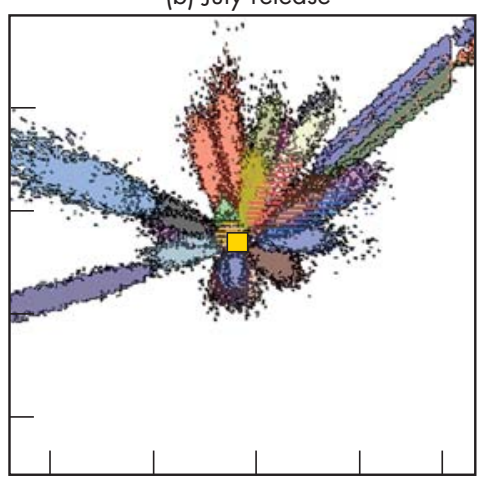

Figure 2. Ensemble of deposition results of 100- to 200-pm particles instantaneously released aloft for 30 days in (a) January and (b) July. Domain shown is $45 \mathrm{~km} \times 45 \mathrm{~km}$. The yellow box marks the release point. 


\section{Project Goals}

The project goals for FY2004 were: finalization of choice of method to produce representative reduction from large datasets; implementation of statistical tools in stages; testing and analysis to investigate the validity and limitations of the tool; and an exit plan and plan for technology transfer.

\section{Relevance to LLNL Mission}

The data reduction tool that we apply for this project will be applicable to other systems both within and outside of LLNL. Efforts from this technology-base project helped to secure funding for a large NAI project beginning in FY2005.

\section{FY2004 Accomplishments and Results}

Different methods to classify wind fields over a location include principal component analysis and cluster analysis.

We used an existing extensive wind-field dataset. These wind data vary on both an hourly basis and on a seasonal basis (Fig. 1). While obvious wind patterns can be discerned, a quantitative method for classifying the wind magnitude, direction and duration is desirable.

Cluster analysis divides a set of observations such that most pairs of observations in the same cluster are more similar to each other than are pairs of observations in two different clusters. Prior to clustering, we used Principal Component Analysis (PCA) to reduce the effective dimension of the dataset.

For this study we used a large set of hind-cast wind-field data and an ensemble of dispersion simulations over a specific region over the entire year of 2003. Using a $K$-means method, we partitioned the entire year's worth of noon and midnight data into distinct clusters which are then characterized by probability distributions that describe spatially varying wind speed and direction. The $u-v$ wind data are plotted in Fig. 3 for the center "release" location of the domain illustrated in Fig. 2.

While clustering was done for the entire year, it is instructive to demonstrate (Fig. 4) how the clustering scheme separates the frequency of wind classes in distal months January and July.

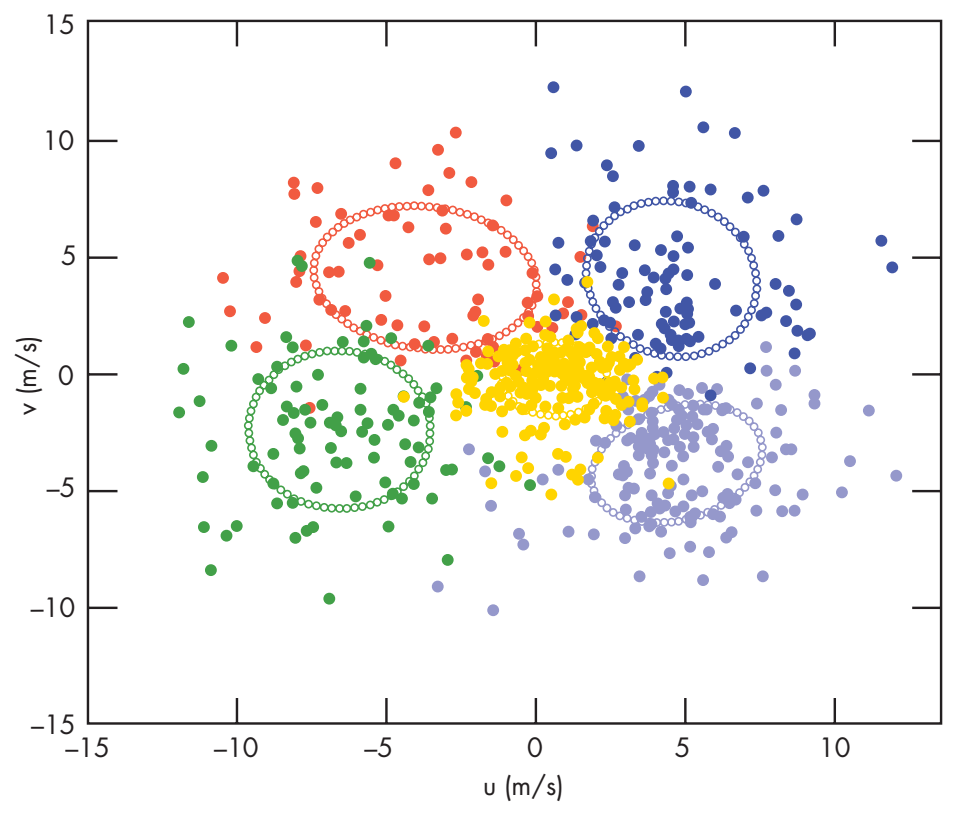

Figure 3. The $2003 \mathrm{u}$-v wind data for 12 p. m. and 12 a. m. wind forecasts at 187-m altitude for the center "release" location. Note 50 th percentile contours for the five clusters are indicated by the colored ellipses.
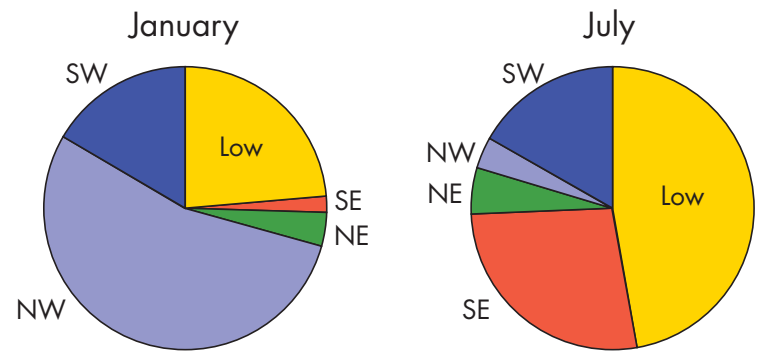

Figure 4. Wind cluster frequency during January and July. January is dominated by westerly winds (70\%) and low winds (25\%). July is dominated by low winds (50\%) and southerly winds (40\%). This indicates a lack of synoptic forcing during the summer months and the dominance of local wind patterns in the region. Westerly synoptic forcing dominates during winter months.

The $K$-means clustering method (with and without PCA pretreatment) is effective in using a heterogeneous high-dimensional multivariate data set to create a manageable set of relatively homogeneous classes which can be characterized stochastically and used in event planning and consequence assessments as well as in sensitivity/uncertainty analyses. The method can easily be modified to account for additional altitudes, a vertical velocity component, and shorter time steps.

\section{Related References}

1. Hardy, D. M., and J. J. Walton, "Principal Components Analysis of Vector Wind Measurements," J. Appl. Meteor., 34, pp. 49-67, 1978.
2. Hodur, R. M., "The Naval Research Laboratory's Coupled Ocean/Atmosphere Mesoscale Prediction System (COAMPS)," Monthly Weather Review, 125, pp. 1414-1430, 1997.

3. Kaufman, L., and P. J. Rousseeuw, Finding Groups in Data: An Introduction to Cluster Analysis, Wiley, 1990.

4. Kaufmann, P., and R. O. Weber, "Classification of Mesoscale Wind Fields in the MISTRAL Field Experiment," J. Appl. Meteor., 35, pp. 1963-1979, 1996.

5. Nasstrom, J. S., G. Sugiyama, J. M. Leone, Jr., and D. L. Ermak, "A Real-Time Atmospheric Dispersion Modeling System," Eleventh Joint Conference on the Applications of Air Pollution Meteorology, Long Beach, California, January, 2000. 


\section{Technologies for Structural Damage Analysis}

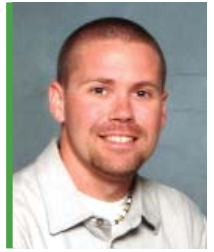

For more information contact Charles R. Noble (925) 422-3057, noble9@|ln.gov

"damage" to the customer, there should be no guessing as to the health of the structure in question.

\section{Project Goals}

The main project objective is to enhance our ability to simulate the response of reinforced concrete structures exposed to extreme loading environments. In addition, we need all-encompassing codes, or codes that can handle all aspects of a blast simulation, for example, in one seamless calculation.

\section{Relevance to LLNL Mission}

The computational tools implemented for this project will decrease the mesh generation time and the computation time. These tools will significantly enhance our ability to analyze the response of reinforced concrete structures for the Department of Homeland Security and Underground Analysis and Planning Systems, and to assess the vulnerability of
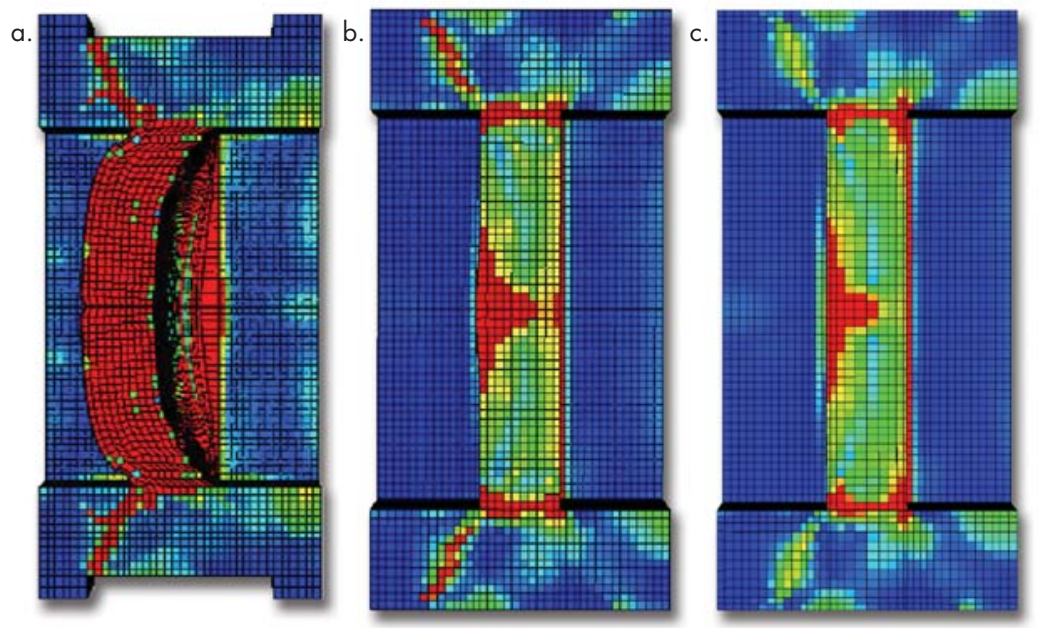

Figure 1. Damage comparison for precision test wall experiment. (a) Test wall with no rebar cage; (b) test wall with rebar explicitly modeled using beam elements in Paradyn; (c) test wall with rebar modeled using new homogenized rebar elements in ALE3D. 


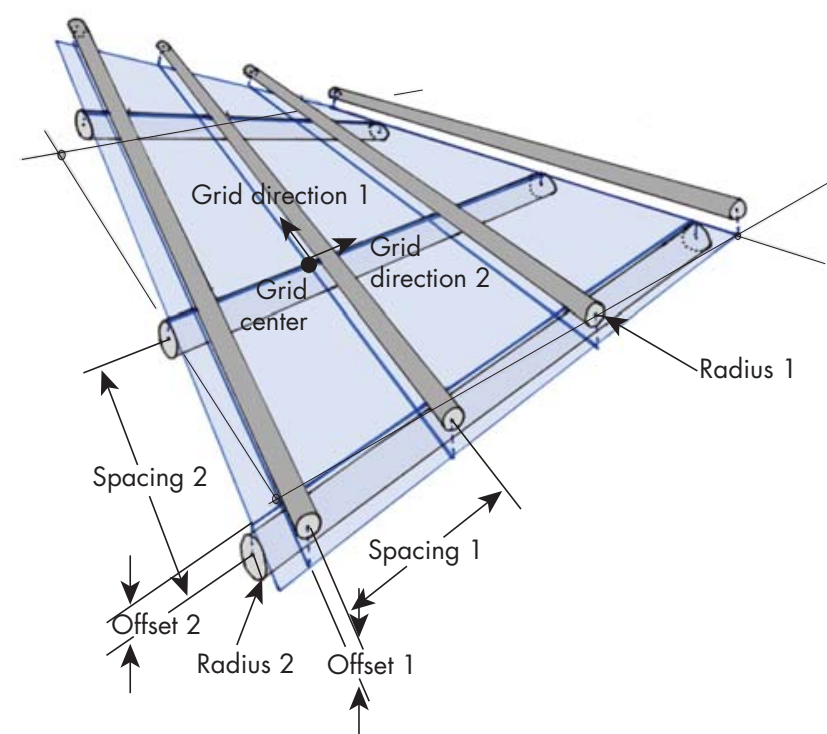

Figure 2. Graphical representation of the rebar detail that FiberGrid will be able to preprocess for ALE3D.
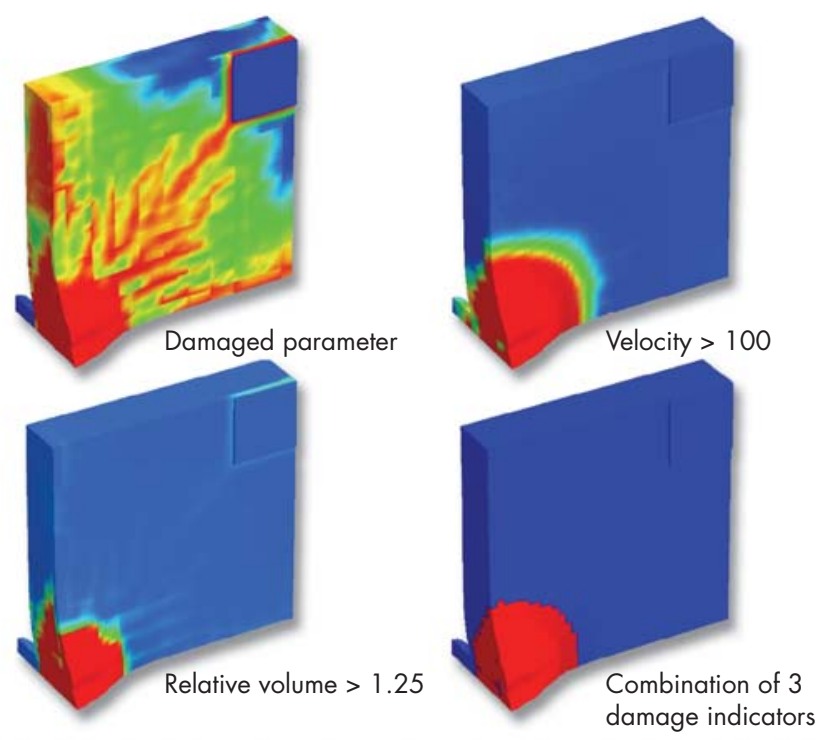

Figure 3. New postprocessing technique in Griz4s that allows the user to combine multiple indicators of damage into one plot. structures to aircraft impact for DOE and NNSA. This project will also help expand LLNL's expertise in advanced computational analysis.

\section{FY2004 Accomplishments and Results}

A composite DTRA Concrete model with homogenized rebar has been coded in ALE3D. This model gives the engineer the capability to simulate strain-rate dependencies, work-hardening, and tensile failure for rebar embedded in concrete. This model will allow advection to occur in the reinforced concrete. Figure 1 shows a comparison of the homogenized rebar model being used on a blast simulation of a reinforced concrete wall, and a comparison with a Paradyn simulation using beam elements to model the rebar.
FiberGrid software (GEN3D) is being generated for use in ALE3D's generator for shaping in rebar grids and also providing the necessary information for the homogenized rebar coding in ALE3D. Figure 2 shows a graphical representation of the type of rebar detail FiberGrid will be able to preprocess.

A new postprocessing technique has been coded into Griz4s for viewing structural failure in reinforced concrete structures. Figure 3 shows how this new technique takes three damage indicators and combines them to give us one that may be an indicator for spall.

The FEAP concrete plasticity model is currently being implemented into NIKE3D and DYNA3D for brick and shell elements.

\section{FY2005 Proposed Work}

The proposed work for FY2005 is to implement DYNA3D's beam elements into ALE3D; implement the homogenized rebar model into DYNA3D; validate the homogenized rebar model for ALE3D and DYNA3D; implement FiberGrid into ALE3D's generator for use with the homogenized rebar model; validate FiberGrid; finish and validate the FEAP concrete plasticity implementation into NIKE3D, DYNA3D, and ALE3D; and validate the new damage metric in Griz4s and implement it into ViSit.

Collaboration with the University of California, San Diego will provide LLNL a suite of test data to validate reinforced concrete models. 


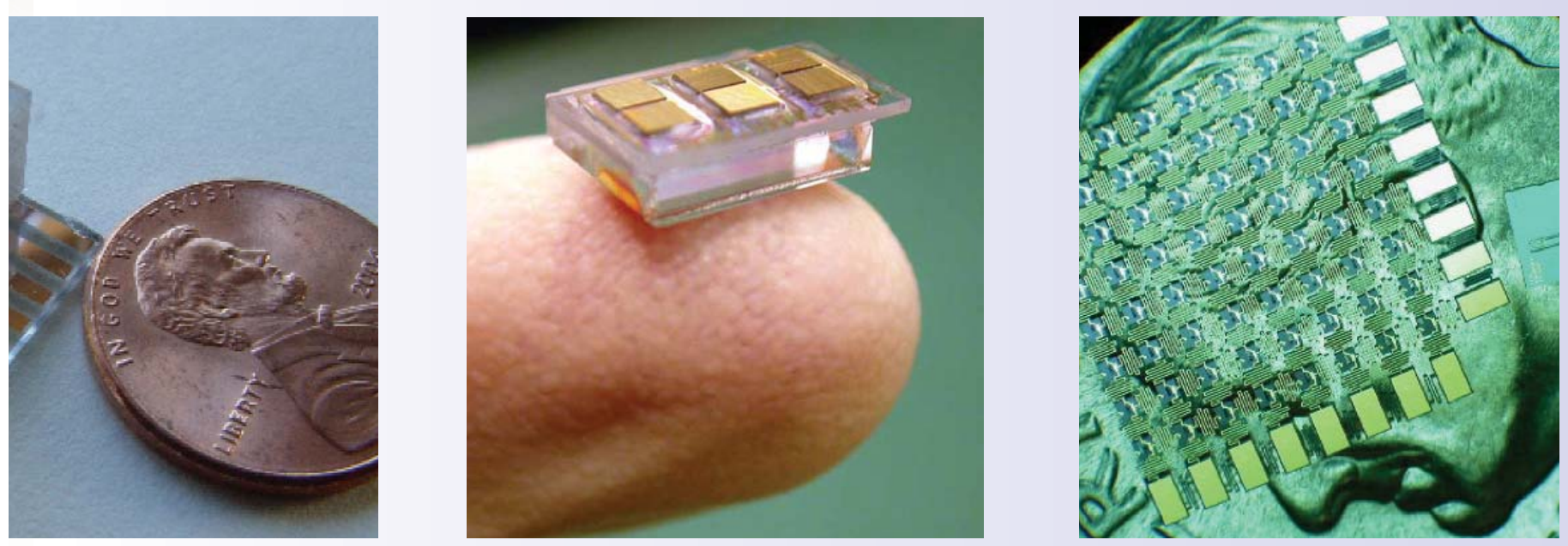

Center for Microtechnology and Nanotechnology 


\section{Contact Stress Distribution Microsensor Array and Optical Force Probe}

any applications require the knowledge of interface stresses for system analyses and control. Examples include the optimization of hip and knee surgery, robotic tactile sensing, internal combustion engine gasket work, and stockpile stewardship demands. An instrument that measures interface stresses must be thin so as not to disturb the stress it measures, and be able to conform to the surfaces it is sandwiched between. These constraints pose numerous technical challenges, to which this work is applied.

\section{Project Goals}

The goal of this project is to improve the measurement of interface stresses. To accomplish this goal, this project demonstrates novel instrumentation. The work is divided into two different technologies:

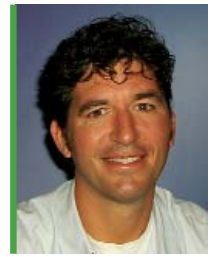

For more information contact Jack Kotovsky (925) 424-3298, kotovskyl@\|ln.gov

electronic and optical measurement systems.

For the electronic system, this year's work seeks to demonstrate all facets of a Contact Stress Sensing Array (CSSA) system. This system is comprised of MEMS stress sensors, an integrated process to interconnect these elements in a 2-D flexible array (see Figs. 1 and 2), control electronics, signal conditioning, and PC-based data acquisition system.

For the optical system, the goal is to demonstrate a novel Optical Force Probe (OFP) and a Modular Optical Assembly necessary for the probe's function.

\section{Relevance to LLNL Mission}

This work demonstrates tools that provide support to LLNL weapons programs and stockpile stewardship, and to many other applications within and beyond LLNL. The effort produces new capabilities

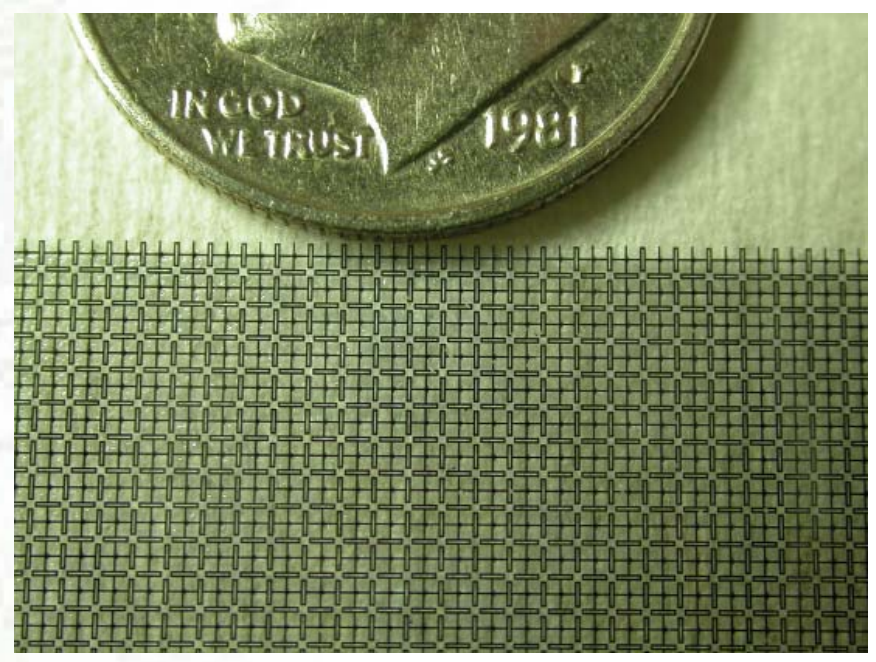

Figure 1. Magnified view of silicon contact stress-sensing array. This prototype demonstrates novel etching and wafer handling of a 1500-sensor array of 60-pm thickness. Approximately 300 sensors with interconnects are shown next to a dime. 
in plasma etching, wet etching of silicon, metallization techniques, nonconventional photolithography, sensors, optical fiber handling, optical fiber alignment, and integrated micro-optomechanical assembly. The work paves the way for LLNL to field novel instruments in stockpile stewardship, and acts as a forcing function for expanded MEMS production capability in the DOE complex.

\section{FY2004 Accomplishments and Results}

Key technical milestones have been achieved this year, including novel techniques in photolithography, metallization, wafer handling, and silicon plasma etching. Large, freestanding, integrated silicon arrays can be produced for the first time. The arrays contain silicon contact stress sensors of $60 \mu \mathrm{m}$ total thickness, with an interconnect scheme allowing complex

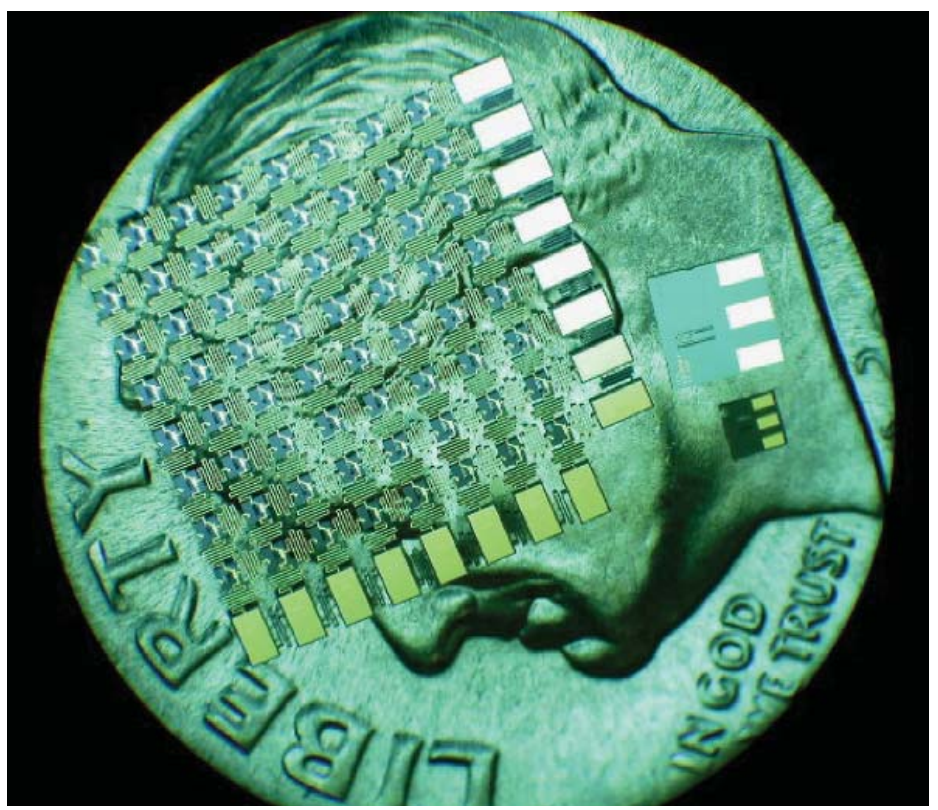

Figure 2. Single and arrayed contact stress sensors shown on a dime. These complete devices are built to measure 0 to $500 \mathrm{psi}$ at a contact interface, and are $50 \mu \mathrm{m}$ in thickness.

Sensor output vs. load

(18k data points/5 load cycles)

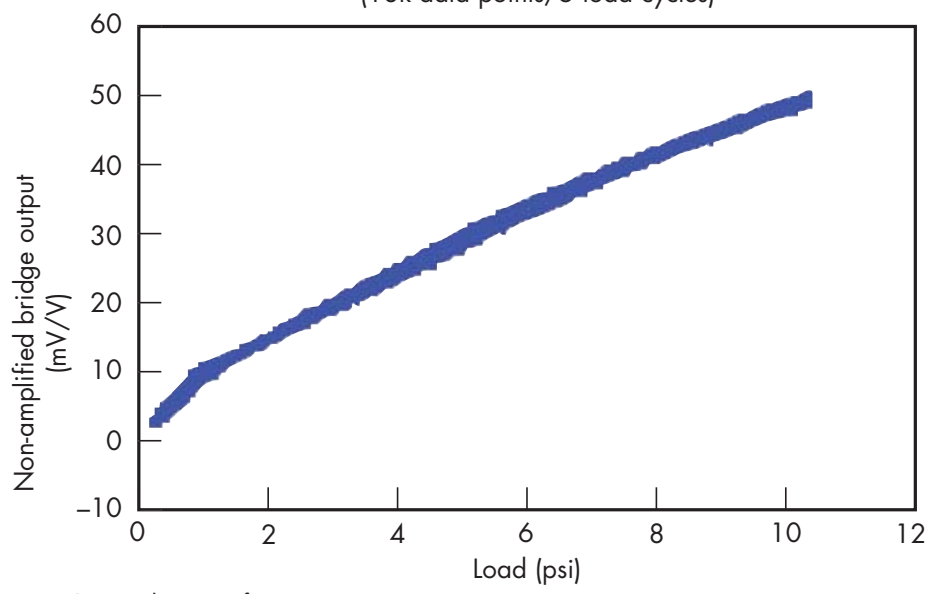

Figure 3. Example output of contact stress sensor.

curvature conformity. This demands array flexibility and extensibility within its plane to achieve two bending radii. Process advances allow the interconnects to be processed independently from the sensing elements, releasing the overall process flow from difficult constraints. Our tools of process monitoring and extensive characterization of the sensing element have improved the sensitivity, accuracy, and linearity of the devices (Fig. 3). The control electronics and signal conditioning have also been realized.

The Modular Optical Assembly has been produced and characterized. Its performance exceeds expectations and provides a robust critical alignment necessary for the OFP's function. The silicon portion of the OFP has also been achieved following advances in plasma etching to produce small, three-dimensionally sculpted devices. The complete OFP is now functioning for the first time.

\section{FY2005 Proposed Work}

Our work on contact stress-sensing arrays will be continued by integrating its components into a complete system. A hybridized silicon rubber package will be produced that accommodates the in-plane extensibility of the array. Once complete, the packaged array, control electronics, and acquisition system will be characterized under a variety of mechanical and thermal loads, and will be improved upon to achieve performance requirements. The OFP will be characterized and its fabrication optimized to provide desired performance requirements. The sensor-fiber assembly will be packaged with necessary strain relief to yield a fieldable instrument. The Modular Optical Assembly will be applied to other optical sensors. 


\section{Direct Wafer Bonding of Gallium-Arsenide to Sapphire}

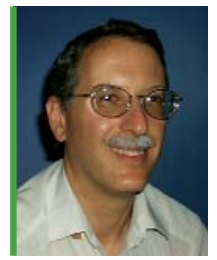

For more information contact Gregory A. Cooper (925) 423-8512, cooper2@\|lnl.gov

His. igh voltage $(>100 \mathrm{~V})$ circuits fabricated on the surface of gallium-arsenide (GaAs) wafers suffer from excessive electrical leakage through the wafer substrate. Formation or placement of isolated GaAs circuit elements on a dielectric substrate has been shown to break the electrical pathway between circuit elements through the substrate and enable operation of highvoltage devices. Bonding of $\mathrm{GaAs}$ to a dielectric substrate using adhesives is not robust enough to survive circuit processing. This work demonstrates an improved method to form a strong and stable bond between GaAs and sapphire that enables the formation of dielectrically isolated high-voltage circuit elements on GaAs using standard IC fabrication techniques.

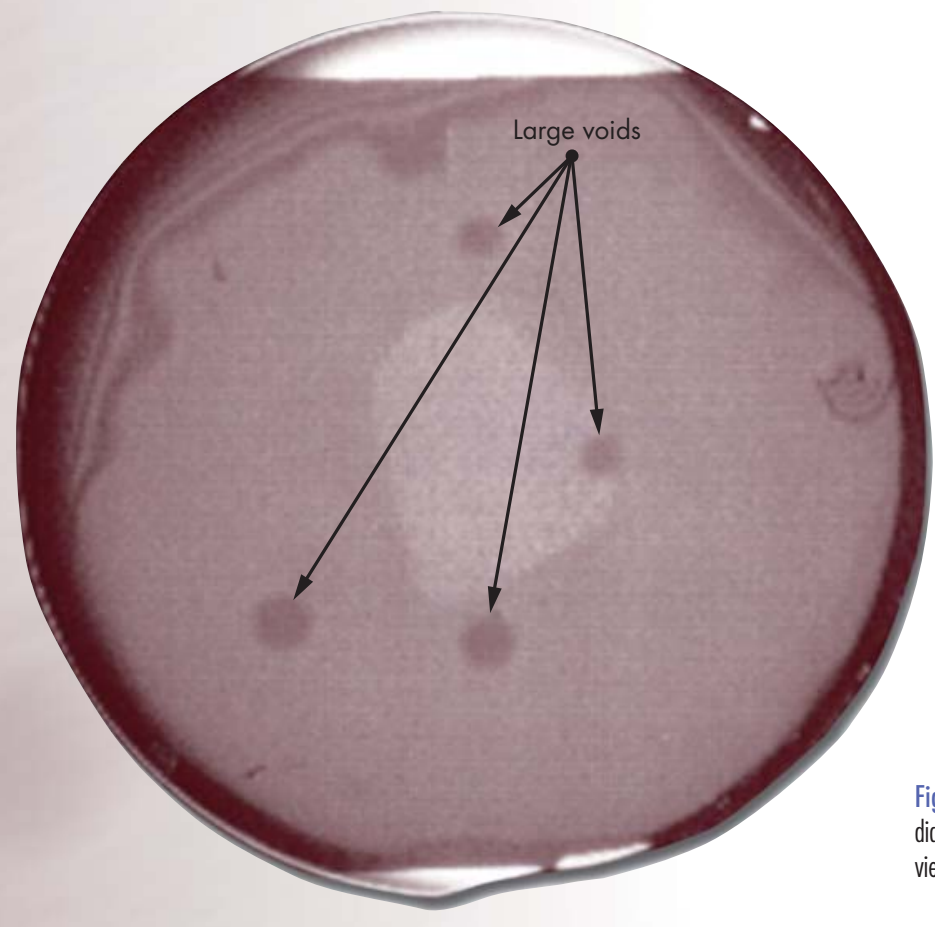

\section{Project Goals}

Our goal was to extend past successful efforts by LLNL's Laser Program (directly bonding GaAs to GaAs) to bonding GaAs to a dielectric material such as sapphire. Our specific goals included direct bonding of 2-in.-diameter wafers with sufficiently large void-free areas to enable the fabrication of complete high-voltage arrays of photodiodes, and bond strengths in excess of $1 \mathrm{~J} / \mathrm{m}^{2}$.

\section{Relevance to LLNL Mission}

Optically powered, high-voltage power supplies have been identified as potential components of advanced firesets for DoD and DOE applications. Compact size, reduced parts count, and inherent electrical isolation are key advantages of photovoltaic power supplies. Direct (covalent) bonding of GaAs to an electrical insulator, such as sapphire, before the circuits are formed in the GaAs would provide a means to use low-cost integrated circuit production techniques to produce high-voltage circuits.

\section{FY2004 Accomplishments and Results}

Wafers of GaAs were prepared with a $1-\mu \mathrm{m}$-thick etchstop layer of aluminumgallium-arsenide (AlGaAs) followed by a $0.1-\mu \mathrm{m}$-thick layer of GaAs epitaxially grown on their surface. The wafers used had surface roughness $<0.2 \mathrm{~nm} \mathrm{rms}, 5$ - to $8-\mu \mathrm{m}$ flatness, and 3- to $8-\mu \mathrm{m}$ bow. The orientation of the GaAs wafers was $<100>$. The orientation of the sapphire wafers was c-plane. 
The bonds were formed using a carefully controlled cleaning and preparation process to minimize any particulate and molecular contamination of the surfaces to be joined. The final step before actually joining a GaAs wafer to a sapphire wafer was to clean each wafer at high temperature in flowing hydrogen gas. Typical GaAs clean was done at $720^{\circ} \mathrm{C}$ as the final part of the epitaxial growth process; the sapphire clean was performed at $500{ }^{\circ} \mathrm{C}$. The pressure was 60 torr and the hydrogen flow rate was about $2 \mathrm{~L} / \mathrm{min}$ through a reactor area of $22 \mathrm{~cm}^{2}$. After both wafers had been treated in hydrogen, they were manually brought into contact with each other at room temperature in an ultra-dry nitrogen environment. Localized mechanical pressure was used to bring the surfaces close enough together to obtain weak contact bonding by Van Der Waals forces. Most large voids, visible as interference fringes through the sapphire wafer, could be successfully collapsed using this technique. The joined pair was again treated to high temperature in hydrogen gas to form a stronger covalent bond.

Visual inspection of the bond interface obtained by bonding at $500{ }^{\circ} \mathrm{C}$ revealed the presence of voids of various sizes ranging from several millimeters to $>100 \mu \mathrm{m}$ in diameter (Figs. 1 and 2). The number of voids depended on void size, with few large voids and many semi-uniform small voids. The small voids were eliminated in subsequent bonds by use of an extended hydrogen purge and clean at $400{ }^{\circ} \mathrm{C}$ of the wafer surfaces in contact but without applied pressure, as well as a lower bond temperature of $400{ }^{\circ} \mathrm{C}$ after pressure contact. The largest bond area free of voids achieved to date is about $1.6 \mathrm{~cm} \times 1.9 \mathrm{~cm}$. One photovoltaic application requires a 1.4-cm-x-1.4-cm area for a complete array.

A test bond was used to obtain a quantitative measurement of the bond strength, using a wedge to pry the wafers apart.

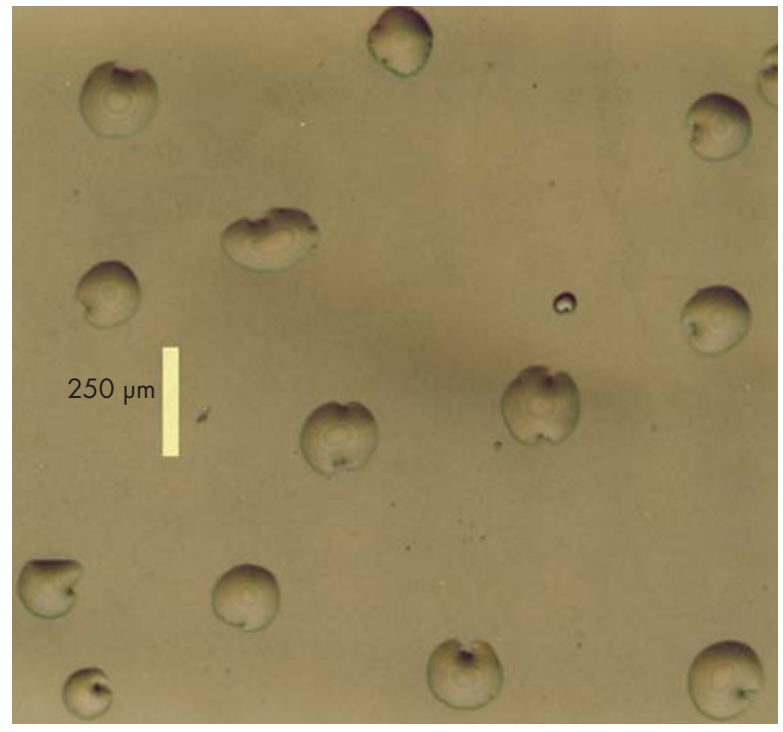

Figure 2. Microvoids visible on surface on first GaAs bond after removal of thick GaAs substrate.

Preliminary analysis of the data indicates a bond strength in the range of $0.71 \mathrm{~J} / \mathrm{m}^{2}$. Successful growth of thick GaAs on a layer of GaAs bonded to sapphire without obvious delamination or cracking is a strong indicator of the strength and robustness of the bond. Since no photovoltaic devices have yet been fabricated on GaAs bonded to sapphire, it is not known if crystalline defects were introduced into the grown GaAs film that would affect photovoltaic performance. 


\section{Electrochemical Detection of Biological Pathogens}

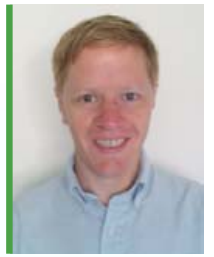

For more information contact David Sopchak (925) 422-2240, sopchak1@\|lnl.gov

active molecules in DNA hybridization studies, and for detectors in capillary gel electrophoresis. While these techniques have exhibited excellent sensitivity, they have not been applied to specific systems for applications to homeland security. This effort will look at the use of custom-patterned electrode materials, which can be directly integrated with capillary gel electrophoresis devices for evaluation against the more proven fluorescent detection techniques.

\section{Project Goals}

The goal of this project is to produce an electrochemical detector for a commercial (Beckman-Coulter) capillary electrophoresis device that will serve as a testbed for further refinement for smaller devices.

previously been investigated using redox

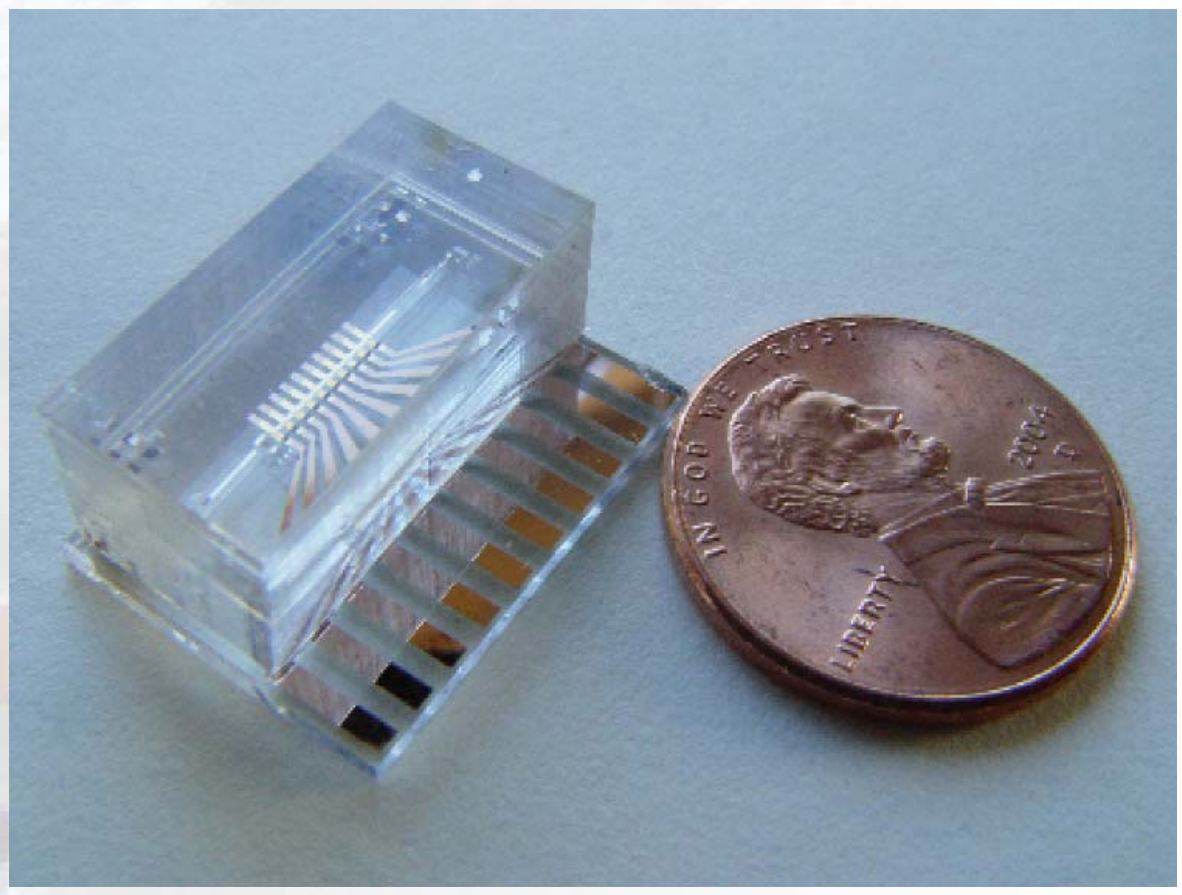

Photograph of prototype electrochemical detector: PDMS on glass with gold electrodes. 


\section{Relevance to LLNL Mission}

The problem of pathogen detection is important for homeland security. Low cost, miniature detection systems will eventually provide new capability to the individual, or to first-response teams, for a variety of field scenarios. The particular problem addressed by this effort couples directly to systems currently being considered for these applications at LLNL.

\section{FY2004 Accomplishments and Results}

In FY2003 we produced the first of the prototype electrochemical detector devices (see figure). ACLARA Inc. provided three anthraquinone-based eTagTM samples for testing. In FY2004, electrochemical hardware (an existing Pine potentiostat, LabJack data acquisition device connected to a Mac) was set up, and software was written for data acquisition. A software workaround for removal of emf-related noise during data acquisition was implemented and was able to lower the apparent noise to $\sim 30 \mathrm{nA}$.

Tests to establish behavior of gold, platinum, and glassy carbon electrodes towards hydroquinone in $1 \mathrm{M}$ potassium chloride were conducted. (Hydroquinone resembles anthraquinone in its electrochemical behavior and has been well studied.) The glassy carbon electrodes displayed the best sensitivity and lowest background current, with detection limits of about $100 \mathrm{nM}$, using conventional techniques.

Electrochemical testing of anthraquinone in aqueous $(1 \mathrm{M} \mathrm{KCl})$ solutions followed, in preparation for using anthraquinone-modified eTags ${ }^{\mathrm{TM}}$. We found that the redox potential of anthraquinone was beyond the cathodic breakdown potential for the solvent, rendering detection of these eTags ${ }^{\mathrm{TM}}$ impossible in aqueous systems.
Testing of the electrochemical detection device that was fabricated in FY2003 was also performed. Detection limits were found to be sub-picomole, limited only by the background noise of the potentiostat being used. Unfortunately, the gold microelectrodes used were not inert enough to withstand the potential at which they were poised, and dissolved completely after an hour's use.

A picoampere-range potentiostat from Bioanalytical Systems was purchased to improve detection limits. To address both the electrode robustness issue and to lower the detection limits, we are fabricating electrodes made from CVD boron-doped diamond films. Borondoped diamond has been shown to have outstanding robustness and excellent sensor properties, including low background current, in electrochemical systems. We believe with these new electrodes and equipment, we can push detection limits to the attomole range and below, which is the state of the art for electrochemical detection.

\section{Related References}

1. Woolley, A., K. Lao, A. Glazer, and R. Mathies, "Capillary Electrophoresis Chips with Integrated Electrochemical Detection," Analytical Chemistry, 70, pp. 684-688, 1998.

2. Backofen, U., F. Matysik, and C. Lunte, "A ChipBased Electrophoresis System with Electrochemical Detection and Hydrodynamic Injection," Analytical Chemistry, 74, pp. 4054-4059, 2002.

3. Sopchak, D., B. Miller, R. Kalish, Y. Avyigal, and X. Shi, "Dopamine and Ascorbate Analysis at Hydrodynamic Electrodes of Boron-Doped Diamond and Nitrogen Incorporated Tetrahedral Amorphous Carbon," Electroanalysis, 14, (7-8), pp. 473-478, 2002. 4. Sopchak, D., B. Miller, Y. Avigal, and R. Kalish, "Rotating Ring-Disk Electrode Studies of the Oxidation of P-Methoxyphenol and Hydroquinone at Boron-Doped Diamond Electrodes," Journal of Electroanalytical Chemistry, 538-539, pp. 39-45, 2002. 


\section{In-Situ Characterization Tools for ECR Plasma Etching}

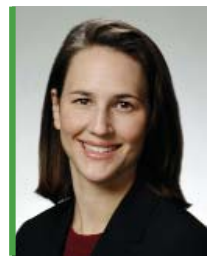

For more information contact Rebecca Welty (925) 423-7389, welty2@lln..gov

have set out to enhance our capabilities to characterize our plasma etching technology by in-situ temperature thermometry and end point detection. These types of analytical characterization tools are needed to provide reproducible, precision depth control as well as insight into the chemical etching mechanisms.

\section{Project Goals}

The goal of this project was to select and install an in-situ wafer surface temperature measurement diagnostic and a plasma chemistry diagnostic on an existing ECR etch tool.

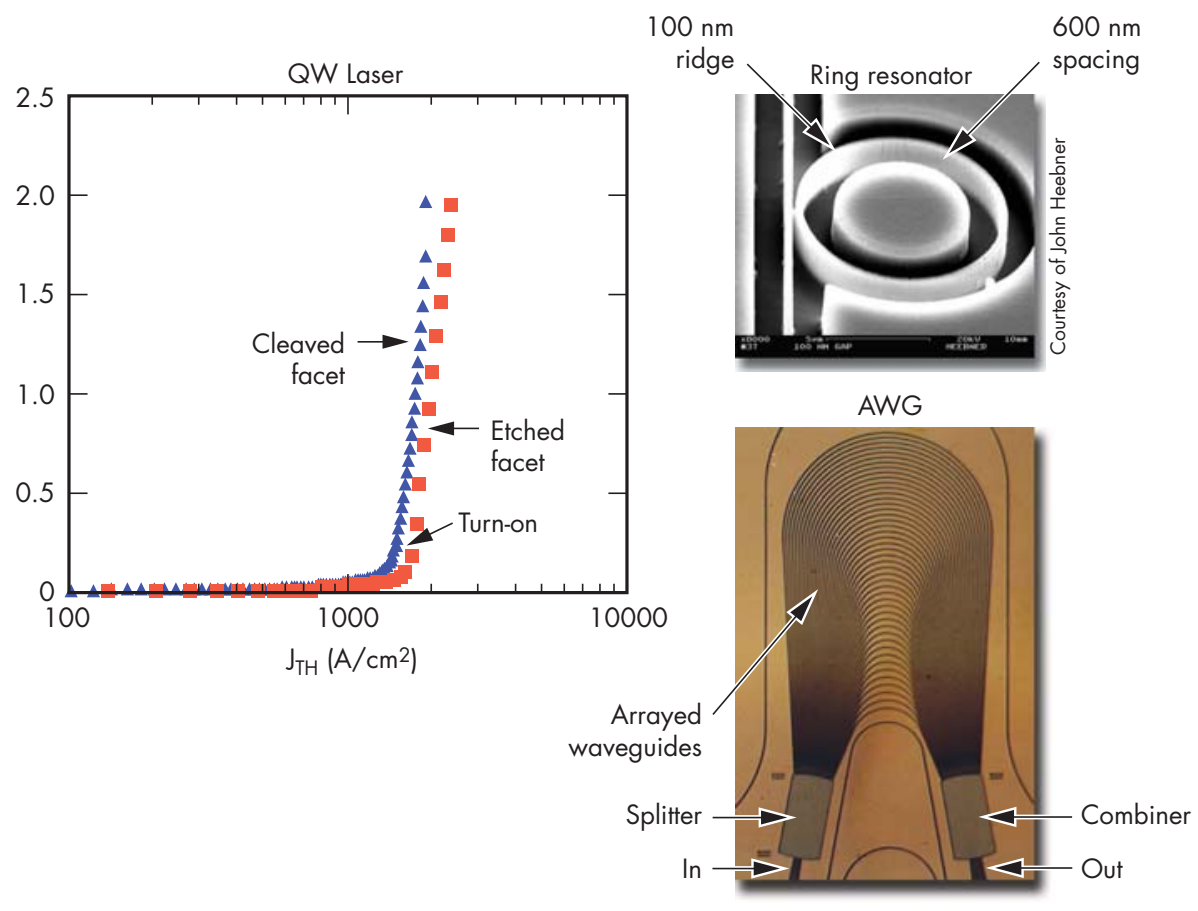

Figure 1. Sample devices we have fabricated, all requiring advanced plasma dry etching, carried out with our ECR system.

\section{Relevance to LLNL Mission}

We support projects that require advanced $\mathrm{InP}$ - and $\mathrm{GaAs}-$ based processing technology. There are many different devices having broad applications that share the same fabrication hurdles. Figure 1 shows representative sample devices, all requiring similar process technology. This technology will allow deployable microfabricated systems for several applications that are at the core of LLNL's national security mission, such as high-speed radiation diagnostic devices for NIF, single-transient recording technologies, and devices for encryption applications. 


\section{FY2004 Accomplishments and Results}

Selecting appropriate temperature and plasma chemistry diagnostic tools was limited by the harsh environment of the etch chamber. Corrosive chemical gases, along with $\mathrm{RF}$ and microwave radiation and strong DC magnetic fields, render electronic technologies useless.

We therefore chose a contacting temperature probe that relies on a well-characterized temperature dependence of a phosphor decay that can be interrogated through an optical fiber and is encased in chemical resistant materials. Figure 2 shows temperature vs. time collected for typical GaAs etch recipes. The ECR plasma has a dramatic impact on the surface temperature of the wafer. Although we had indirect indications of the peak wafer temperatures in the past, this was our first measurement of the actual time evolution of the temperatures. This information will make further optimization possible.

To characterize the chemical composition of the plasma, and thus indirectly measure the chemical composition of the wafer surface currently being etched, we selected a plasma emission spectrometry tool. This tool collects the light emitted by the plasma through a viewport on the etch chamber and separates the light into its various spectral components. By analyzing the variations of the emission spectrum as a function of time in real time (Fig. 3), the boundary between different layers on the sample can be identified and used to control the etch process. The installation and calibration of the analytical tools is complete. The final requirement is to create endpoint detection recipes based on the identification of the measured spectrum for the various devices that are being fabricated.

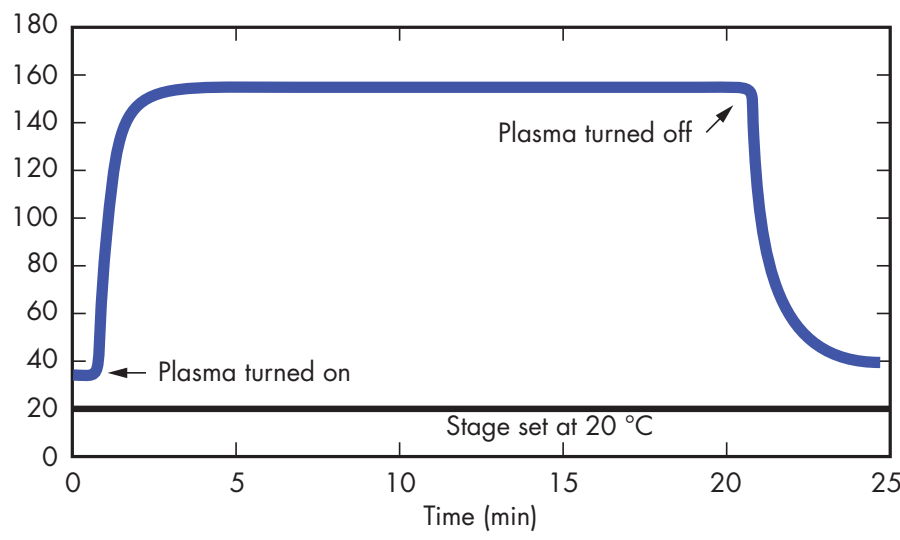

Figure 2. Temperature of surface of GaAs wafer during ECR etch process with He backside cooling, as recorded by optical contact thermometry.

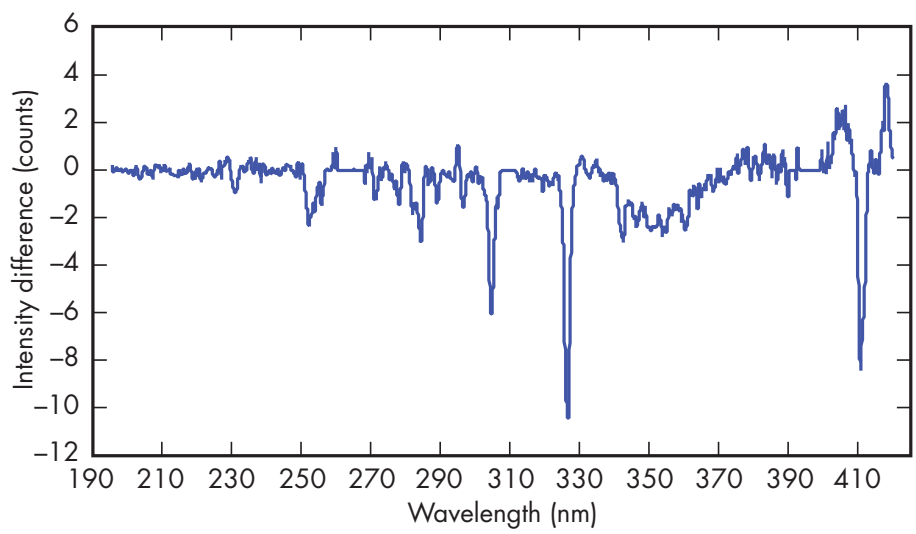

Figure 3. Calculated difference between spectrum recorded during $\ln P$ etch and another spectrum recorded during InGaAsP etch. Key wavelengths of interest are clearly visible as sharp peaks.

\section{Related References}

1. Welty, R. J., C. E. Reinhardt, I. Y. Han, Y. Du, and S. J. Yoo, "Chlorine-Hydrogen ECR Etching of InGaAsP/InP," International Semiconductor Device Research Symposium, Washington D. C., December 2003.

2. Thomas, S., III, E. W. Berg, and S. W. Pang, "In Situ Fiber Optic Thermometry of Wafer Surface Etched with an Electron Cyclotron Resonance Source," American Vacuum Society, 14, (3), pp. 1807-1811, 1996.
3. Thomas, S. III, K. K. Ko, and S. W. Pang, "Monitoring InP and $\mathrm{GaAs}$ Etched in $\mathrm{Cl}_{2} / \mathrm{Ar}$ Using Optical Emission Spectroscopy and Mass Spectrometry," American Vacuum Society, 13, (3), pp. 894-899, 1995.

4. Hebner, G. A., P. A. Miller, and J. R. Woodworth, "Overview of Plasma Diagnostic Techniques," in Handbook of Advanced Plasma Processing Techniques, R. J. Shul and S. J. Pearton, Eds., Springer, Germany, pp. 145-200, 2000. 


\section{Microfluidic Backbone and Components Using New Fabrication Capability for CBNP Applications}

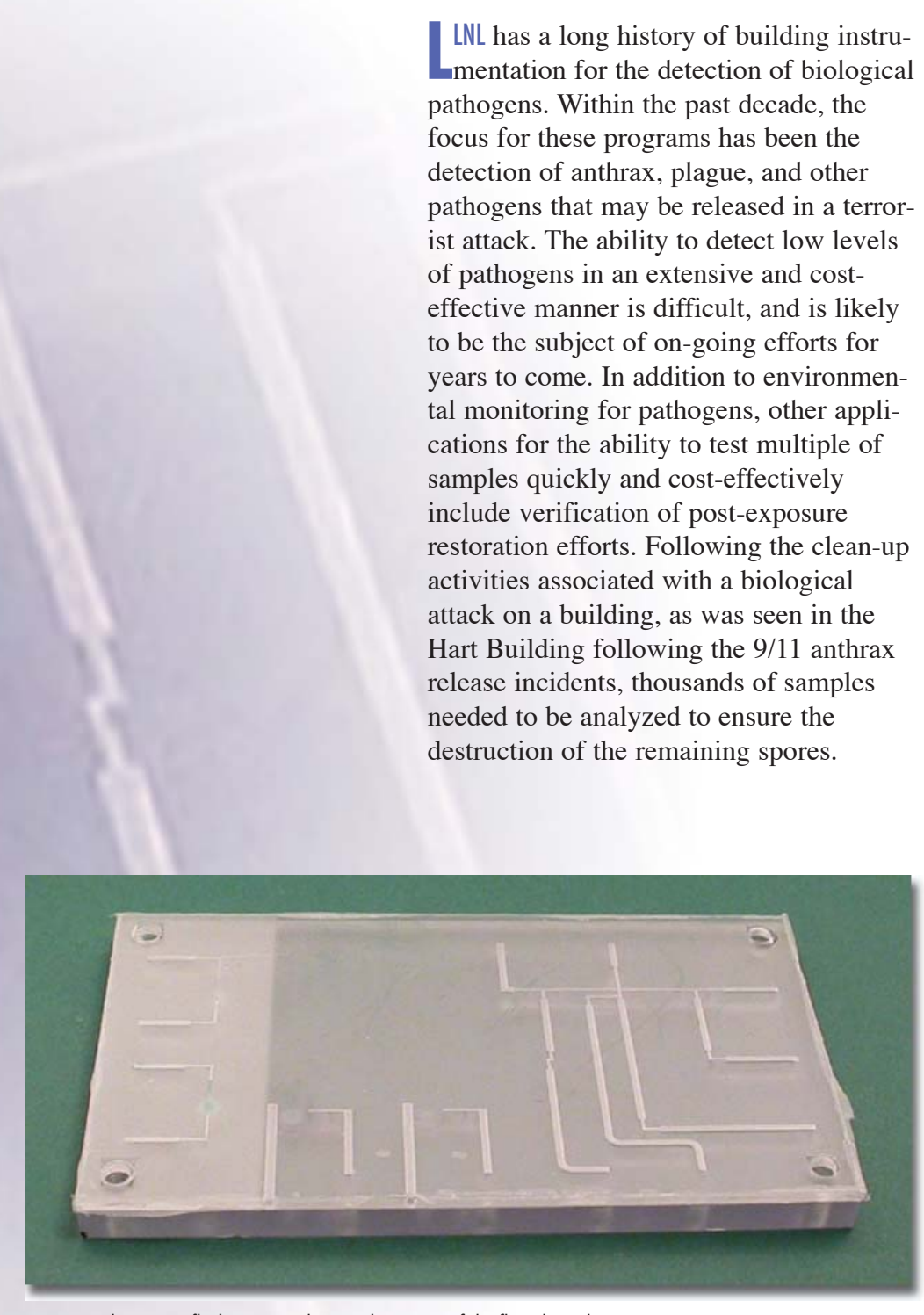

Figure 1. Plastic microfluidic system, showing the pattern of the flow channels.

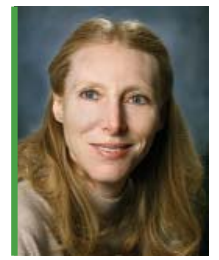

For more information contact Robin Miles (925) 422-8872, miles7@|ln..gov

\section{Project Goals}

Our goal is to provide the capability of producing low-cost plastic-based microfluidic modules. In addition to this module, a latching actuator and a spring-actuated pump were built for use in portable instruments. Also, a module for dispensing a set of ten liquid chromatography samples simultaneously was built to demonstrate usefulness for a current application.

\section{Relevance to LLNL Mission}

As an active participant in these and other programs for biological instruments for pathogen detection, LLNL needs the capability of producing low-cost plasticbased microfluidic modules similar to those published in the literature (see Related References).

\section{FY2004 Accomplishments and Results}

The microfluidic module was built through the machining of acrylic. Figure 1 shows the pattern of the flow channels. The actuation mechanisms consist of pneumatically actuated membrane valves. A prototype was built to demonstrate the functions of metering, mixing, and pumping. The prototype initially used membrane material similar to the cited references. It was found through testing that the device suffered failings, consisting primarily of leaky valves and other seals, caused by normal chipping at the edges of the holes, scratches, bowing plates, and the excessive force required to create a gasket seal over a large, irregular surface.

Such difficulties were thought to limit the usefulness in cost-sensitive applications and where larger formats were needed. 


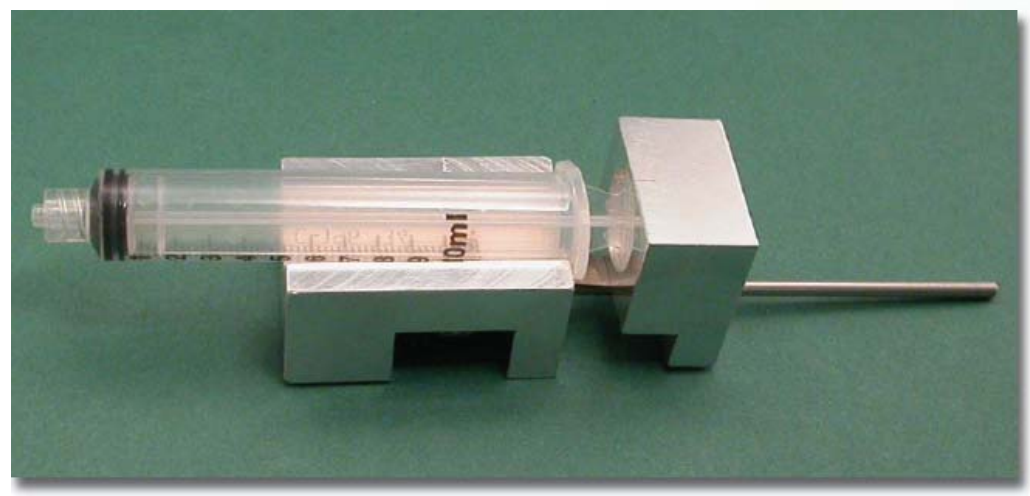

Figure 2. Spring-loaded pump for portable instrumentation.

Therefore, a new membrane scheme was implemented which used adhesive material to form the valve seats. When compressed, the thick membrane material was able to seal the valve. Peristaltic pumps were formed by depressing larger areas of adhesive-inhibited membrane material.

In portable bio-instrument applications, pneumatically actuated valves and pumps are inconvenient at best. The compressed air supply would have to be provided by either an air compressor or by transporting a small gas cylinder with each instrument built using this technology. To make this technology more appealing in cases where field-portable equipment is desirable, as may be the case for the first-responder community, a low-power latching valve was prototyped. The actuator mechanism is based on nickel-titanium shape memory alloy material. Two springs made of this alloy were set opposite each other to form the actuator, with a magnet providing the latching mechanism. This actuator was able to provide $1 \mathrm{~N}$ of force. A constant force pump (Fig. 2) was also demonstrated which could be used as a portable liquid source.

In addition to the basic fluidic module, a special fluidic module was built as a demonstration unit for a particular current application. The chosen application was a thin-layer chromatography unit to test numerous samples simultaneously for the presence of explosive material. In this case, ten simultaneous samples of a specific sample volume were delivered to a chromatographic plate. The device needed to be extremely easy to operate and extremely low power. A module based on capillary action was built and tested. The module wicked in the desired sample volume and dispensed it similarly.

\section{Related References}

1. Anderson, R., X. Su, G. Bodgan, and J. Fenton, "A Miniature Integrated Device for Automated Multistep Genetic Assays," Nucleic Acids Research, 28, (12), 2000

2. Anderson, R., G. Bogdan, Z. Barniv, T. Dawes, J. Winkler, and K. Roy, "Microfluidic Biochemical Analysis System," Proceeding of Transducers '97, Chicago, Illinois, pp. 477-480, June 1997. 


\section{Miniature Multichannel Fiber-Optic Signal Conditioner}

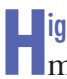
igh-fidelity testing requires miniature, minimally invasive sensors and readout systems to go inside test assemblies. Sensors also need to be optically read out to reduce exposure of sensitive components to electrical energy. Several different sensors are being built to measure parameters such as acceleration, strain, displacement, pressure, and temperature, at various locations within test assemblies. A variety of commercial and custom-made Fabry-Perot interferometric sensors are available, but these signal conditioners (readout systems) are big and bulky, usually rack mounted, and unacceptable for LLNL applications.
\end{abstract}

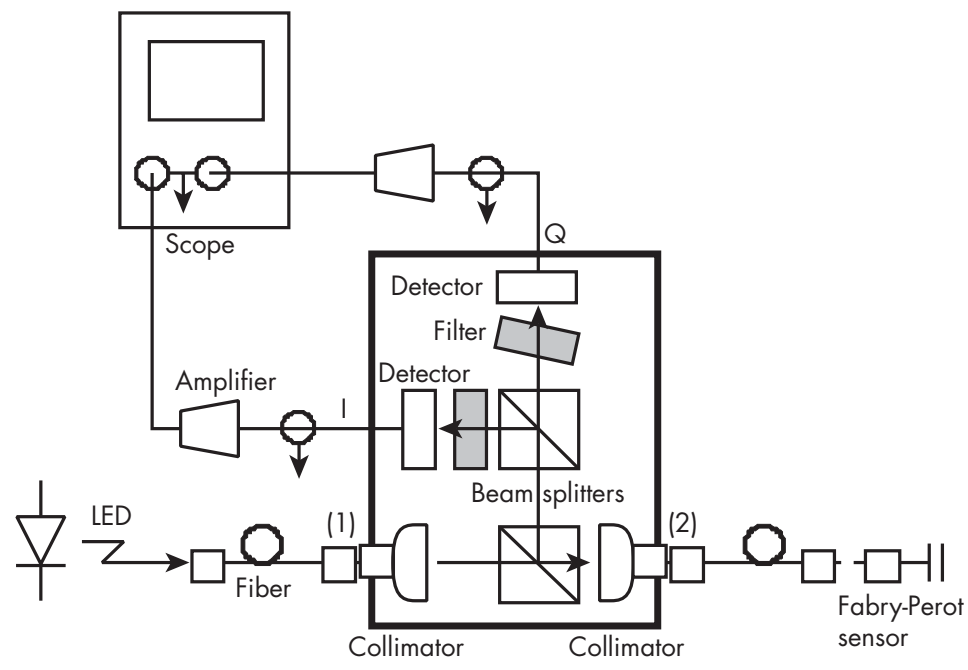

Figure 1. Schematic diagram of basic components for $\mathrm{I} / \mathrm{Q}$ signal analysis.

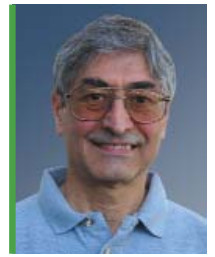

For more information contact Michael D. Pocha (925) 422-8664, pocha1@\|nl.gov

\section{Project Goals}

This was the second and final year of a project with two main goals: 1) finish implementing a miniature three-channel fiber-optic readout conditioner; and 2) begin reduction to practice of technology for a miniature absolute conditioner for applications where absolute measurements are necessary. We completed our first goal and made significant progress on the second.

\section{Relevance to LLNL Mission}

High-fidelity flight tests are required by the weapons stockpile stewardship mission to ensure weapon viability without underground testing.

\section{FY2004 Accomplishments and Results}

The I/Q Concept. For relative measurements we chose the in-phase and quadrature I/Q technique, which takes advantage of the virtually sinusoidal shape of interference fringes that result from fiber-optic sensors. Figure 1 is a schematic of the main components. A phase-modulated light beam is reflected from a Fabry-Perot sensor split and directed to two filters, which look at a narrow portion of the spectrum (about $10 \mathrm{~nm}$ wide), making fringes visible as an amplitude modulation. One filter produces the in-phase signal. The second filter is tuned to a slightly different part of the spectrum and produces a fringe pattern that is $90^{\circ}$ out of phase (in quadrature). As the Fabry-Perot sensor changes dimension due to a physical stimulus, the corresponding change in the phase angle allows reconstructing the temporal behavior of the phenomenon being measured. 


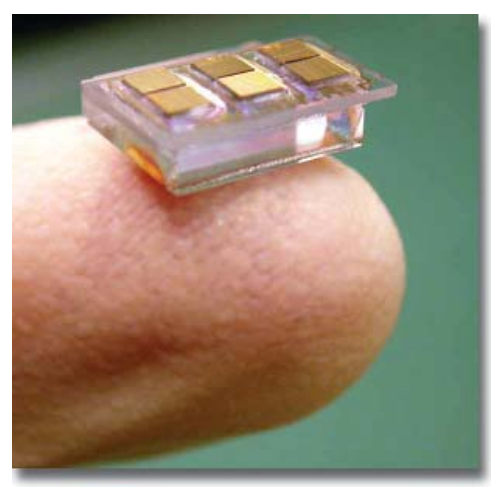

Figure 2. Optical elements on a human finger.

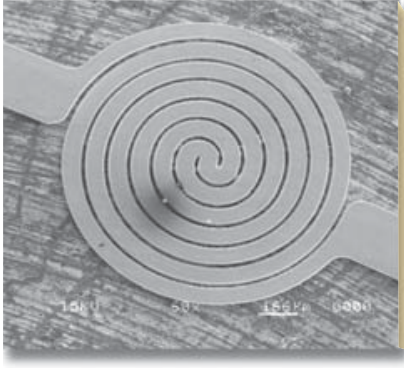

Filament-1 $\mathrm{mm}$ diameter

Figure 3. Miniature filament light source for coupling to optical fiber.
I/Q Implementation. The optical path can be implemented entirely within a small slab of glass ( $3 \mathrm{~mm}$ wide $¥ 2 \mathrm{~mm}$ thick $¥ 10$ $\mathrm{mm}$ long). Additional channels are allowed by increasing the width of the glass and adding detectors in parallel. Figure 2 is a photo of the complete three-channel optical element sitting on a human finger. It includes all the components described above except the collimators. State-of-theart technologies were used for nanostructured thin film filters, chip detectors and flip-chip mounting and connection.

To test operation, we mounted a FabryPerot accelerometer on a shaker table and recorded the signal using our conditioner, an external oscilloscope, and a computer.

Absolute Conditioner. We have been exploring techniques for miniaturization of absolute signal conditioning. We built a laboratory bench system that uses a light bulb as a source and a piezoelectrically-tuned filter to perform the signal capture transform. We are also collaborating with NASA to implement a miniature incandescent filament light source. LLNL made key contributions to packaging and hermetic sealing.
A photo of one of our devices is shown operating in Fig. 3. With NASA, we are preparing to transfer this technology to the commercial sector.

\section{Related References}

1. Furstenau, N., M. Schmidt, W. J. Bock, and W. Urbanczyk, "Dynamic Pressure Sensing with a Fiber-Optic Polarimetric Pressure Transducer with Two-Wavelength Passive Quadrature Readout," Applied Optics, 37, (4), February 1998.

2. Lo, Y., J. S. Sirkis, and C. Chang, "Passive Signal Processing of In-Line Fiber Etalon Sensors for High Strain-Rate Loading," Journal of Lightwave Technology, 15, (8), August 1997.

3. Schmidt, M., and N. Furstenau, "Fiber-Optic Extrinsic Fabry-Perot Interferometer Sensors with Three-Wavelength Digital Phase Demodulation," Optics Letters, 24, (9), May 1, 1999.

4. Tuma, M., K. King, L. Kim, R. Hansler, E. Jones, and T. George, "MEMS Incandescent Light Source," Proceedings of SPIE, 4134, 2000.

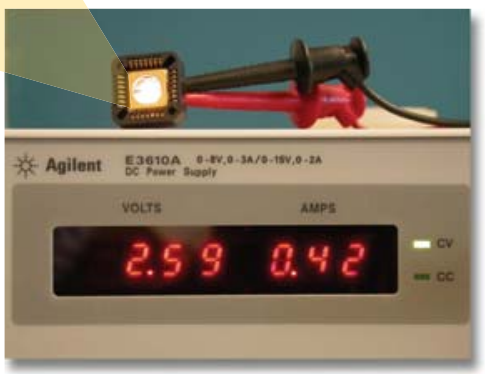

\section{FY2005 Proposed Work}

Seventy percent of the volume of our current three-channel conditioner comprises commercial collimators. By customizing, we can reduce the volume of the collimators by a factor of two or more. Additionally, we would like to include the electronics for amplifying, digitizing, and multiplexing the analog signals on a programmable logic chip incorporated into our package. Finally, we need to miniaturize and implement an absolute measurement conditioner. 


\section{Three-Dimensional Microlithography System}

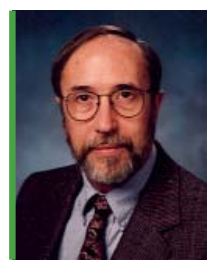

For more information contact Vincent Malba ((925) 422-7296, malbal@|lnl.gov

Another limitation was that motors could not be started at exactly the same time. To use a simple example, this meant that diagonal lines in the x-y plane were executed as stair steps. Modern motor control systems allow simultaneous movement of multiple stages so that smooth diagonals, ovals, and other shapes can be drawn.

\section{FY2004 Accomplishments and Results}

We have assembled a modern, fully 3-D, sub- $\mu \mathrm{m}$-accuracy motion system driven by a commercial CAD/CAM package. This 3-D microlithography system (3D MLS) functions as both a lathe and a five-axis milling machine, each using the focused laser as the "cutting tool," to expose photoresist.

The upgrade of the 3D MLS involved the replacement of the original custom hardware and software with a standard motion-control hardware package, together with commercially available 3-D CAD/CAM software, licensed to LLNL. The new software outputs standard G-code, which the new hardware executes in the same fashion as a five-axis CNC milling machine.

A true 3-D MLS is our final product. It is a unique capability, and is expected to generate considerable interest and support. It dramatically increases our 3-D patterning 
capability, making it applicable to surfaces and structures that could not be patterned previously. As technology demonstrations, we have patterned helices and racetrack coils on $<400-\mu$ m-diameter cylindrical surfaces (for NMR and MRI applications) and simple periodic structures on spherical or elliptical surfaces of radius of order $1 \mathrm{~mm}$ (for NIF physics targets).

We have installed new motion-control hardware, including a Pentium computer, a Delta Tau microprocessor control card, motor amplifiers and motor controllers for up to eight degrees of freedom. A 3-D CAD/CAM package was installed, and initial handshaking software linking it to the motion-control hardware was put in place. Also, x-, y- and z-translation stages (existing equipment) were installed and wired. Requirements for high-precision theta in $\mathrm{x}$ and $\mathrm{z}$ axes were ascertained,

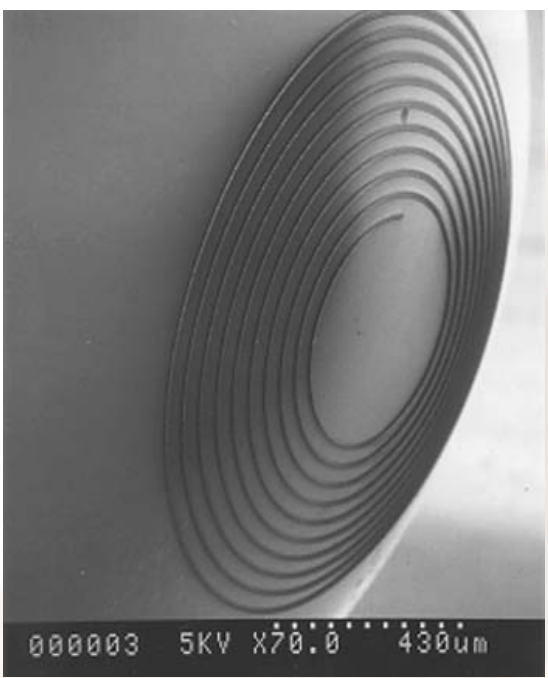

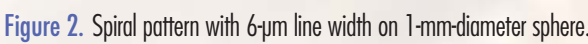

and stages with sufficient speed and precision were purchased and installed. An initial version of a Visual Basic GUI front end for the PC was written. We also assembled a new optical system with versatility as well as minimum feature size under various motions. Improvements in the optical system have produced a "cut size" of $2 \mu \mathrm{m}$ with thin resist.

Creating fixtures for various workpieces is also a significant issue when precise knowledge of the exact position of the part is required. For example, we have made significant progress in handling and manipulating $240-\mu \mathrm{m}$-diameter flexible tubes on which NMR coils are fabricated. We are now confident of our ability to fabricate $100-\mu$ m-diameter NMR tubes for DOE and DHS in the coming year.

Figures 1 to 3 are examples of our work.

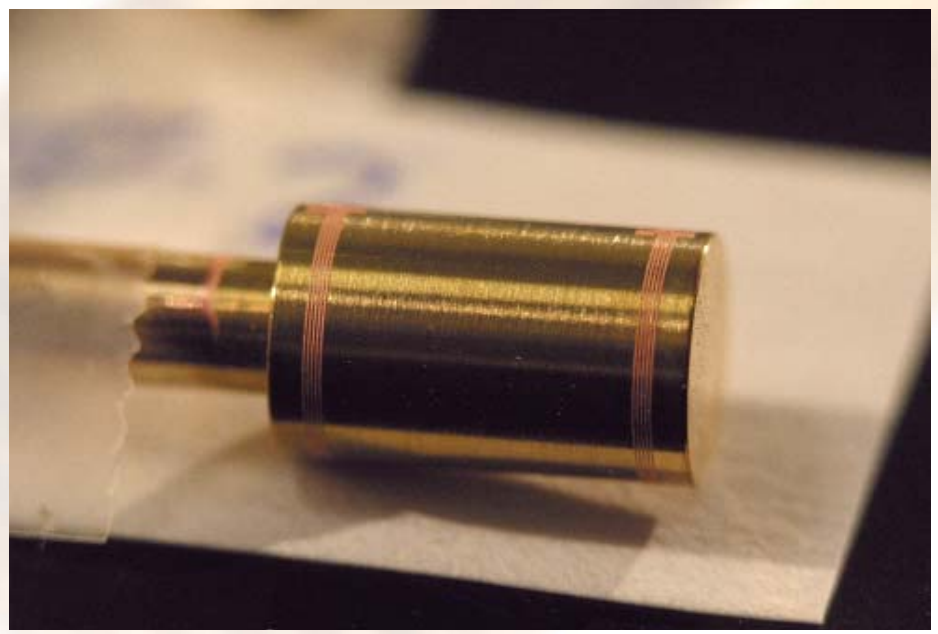

Figure 3. 6-mm-0D brass mandrel patterned with copper to simulate "Hohlraum" heater wires. 

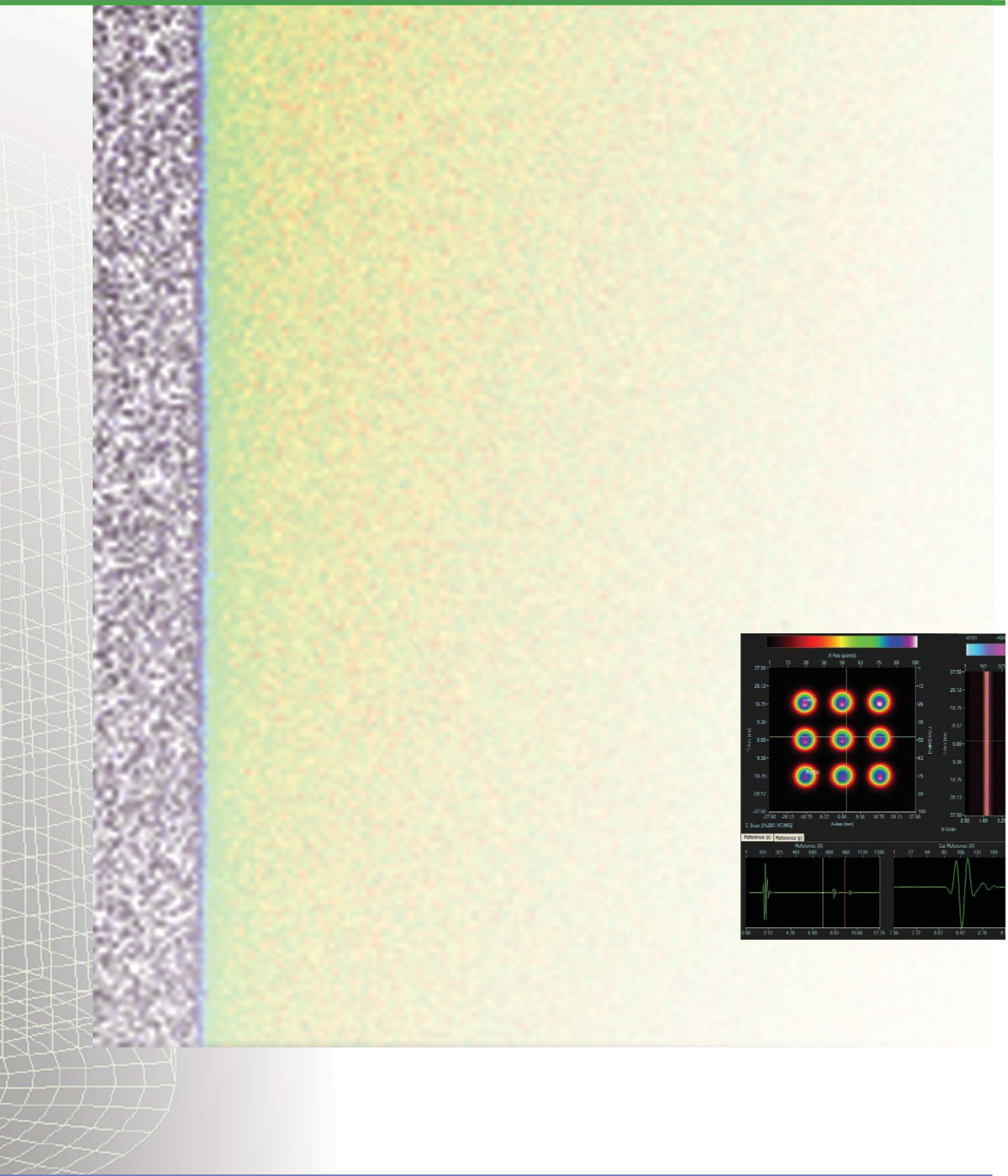

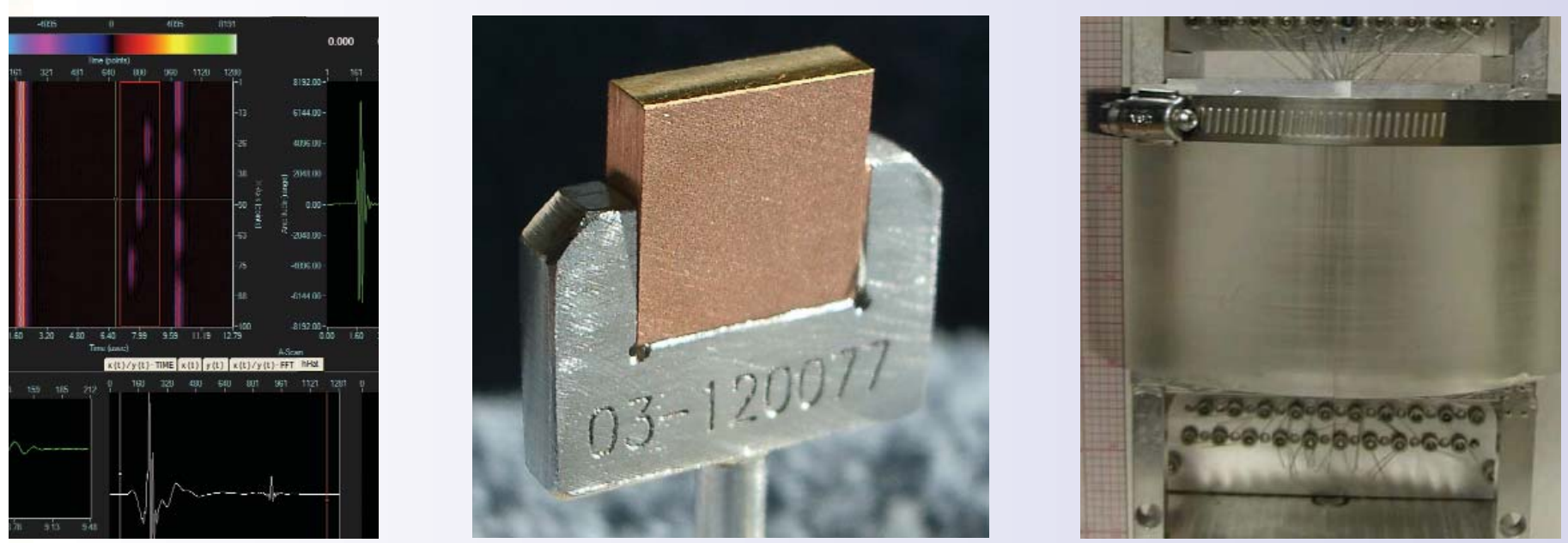

\section{Center for \\ Nondestructive Characterization}




\section{Application of Nondestructive Evaluation to Finite-Element Analysis Coupling for Numerical Analysis}

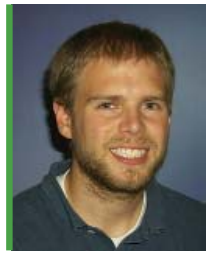

For more information contact Edwin J. Kokko (925) 422-4246, kokko1@lln..gov

feature extraction); analysis mesh generation; running the analysis code (such as mechanical, electromagnetic, thermal, and hydro); and finally, interpreting the results.

\section{Project Goals}

The first of the two project goals was to collect and process NDC data sets for a known "phantom" object that was conceptualized and built specifically to demonstrate the process using real data. The focus of this exercise was to uncover issues with existing methods for manipulating and condensing real characterization data sets into analysis models.

The second goal was to build and document a technology roadmap that highlights these issues and makes recommendations that enable engineering and physics analysts to regularly model asbuilt objects in the near future.

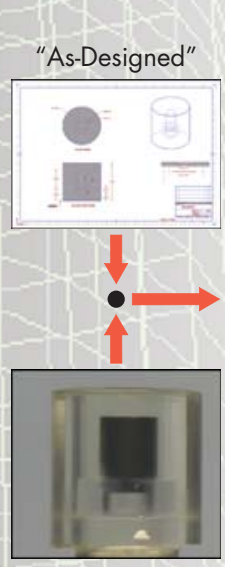

"As-Built"
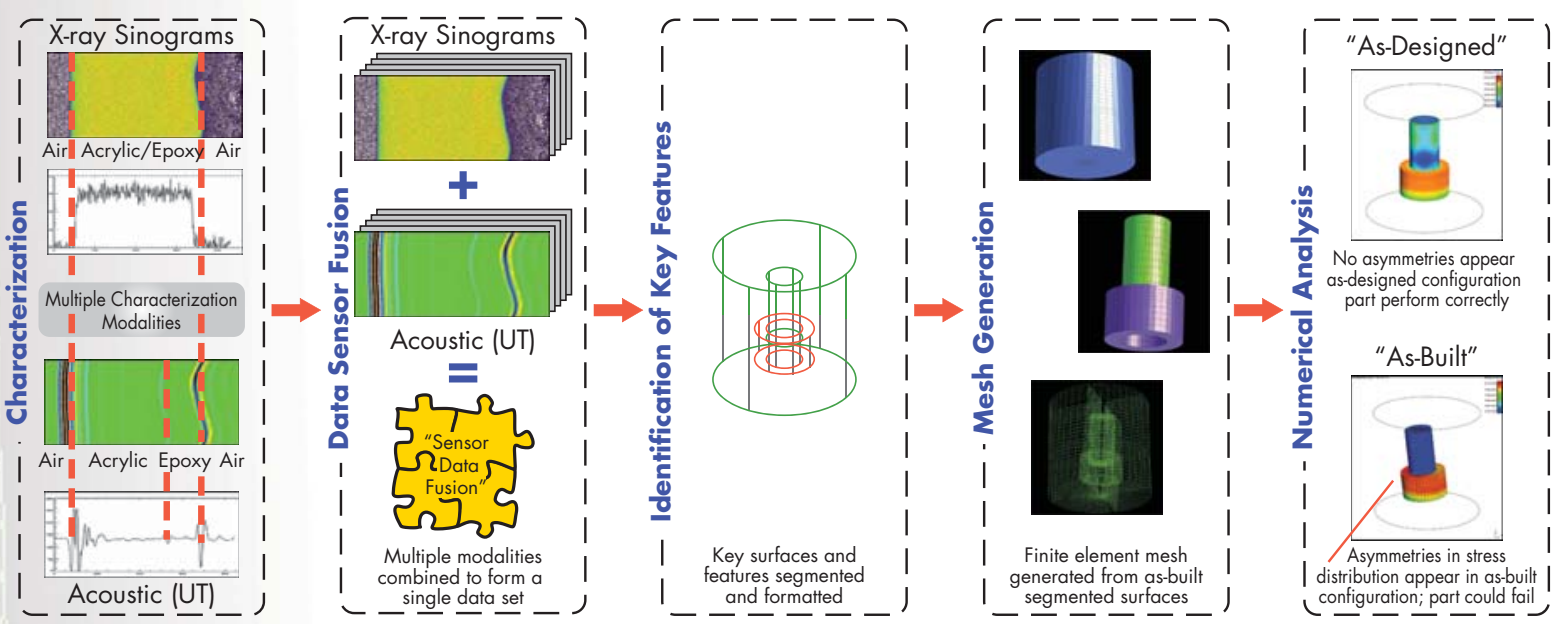

Figure 1. As-built modeling: key steps applied to cylindrical phantom object. The cylindrical phantom was characterized by x-ray CT and acoustics The data sets were fused and used to identify and extract features and surfaces. This data was then used to generate as-built meshes that were structurally analyzed. The analysis of the as-built mesh showed the part had significantly asymmetric stress distributions when loaded. 


\section{Relevance to LLNL Mission}

LLNL projects that would benefit from further improvement of this methodology include NIF and the Stockpile Stewardship Program. For example, characterization data for precision-machined targets could potentially be integrated into pre-test analysis simulations to better understand NIF high-energy-density experiments. This could allow an analyst to isolate and determine if manufacturing process defects might influence experimental results. Similarly, this methodology could be applied in the analysis of existing weapon components and assemblies to build confidence in the certification process.

\section{FY2004 Accomplishments and Results}

This fiscal year, an ultrasonic NDC data set was collected to complement an existing $\mathrm{x}$-ray computed tomography $(\mathrm{CT})$ data set of a known "phantom" part in a controlled experiment designed to demonstrate the strengths and weaknesses of $\mathrm{x}$ ray $\mathrm{CT}$ and ultrasonic testing (UT) imaging. These data sets successfully provided an illustration of the advantages of fusing data sets collected from multiple modalities to form a composite that provides more information than the individual data sets themselves. This exercise involved extensive CT and UT signal and image processing work, including the implementation of a manual co-registration scheme and a preliminary data sensor fusion scheme.

Also this fiscal year, the project team demonstrated the as-built methodology when applied to a real NDC data set collected for a NIF high-energy-density spherical reference standard target (Fig. 2).

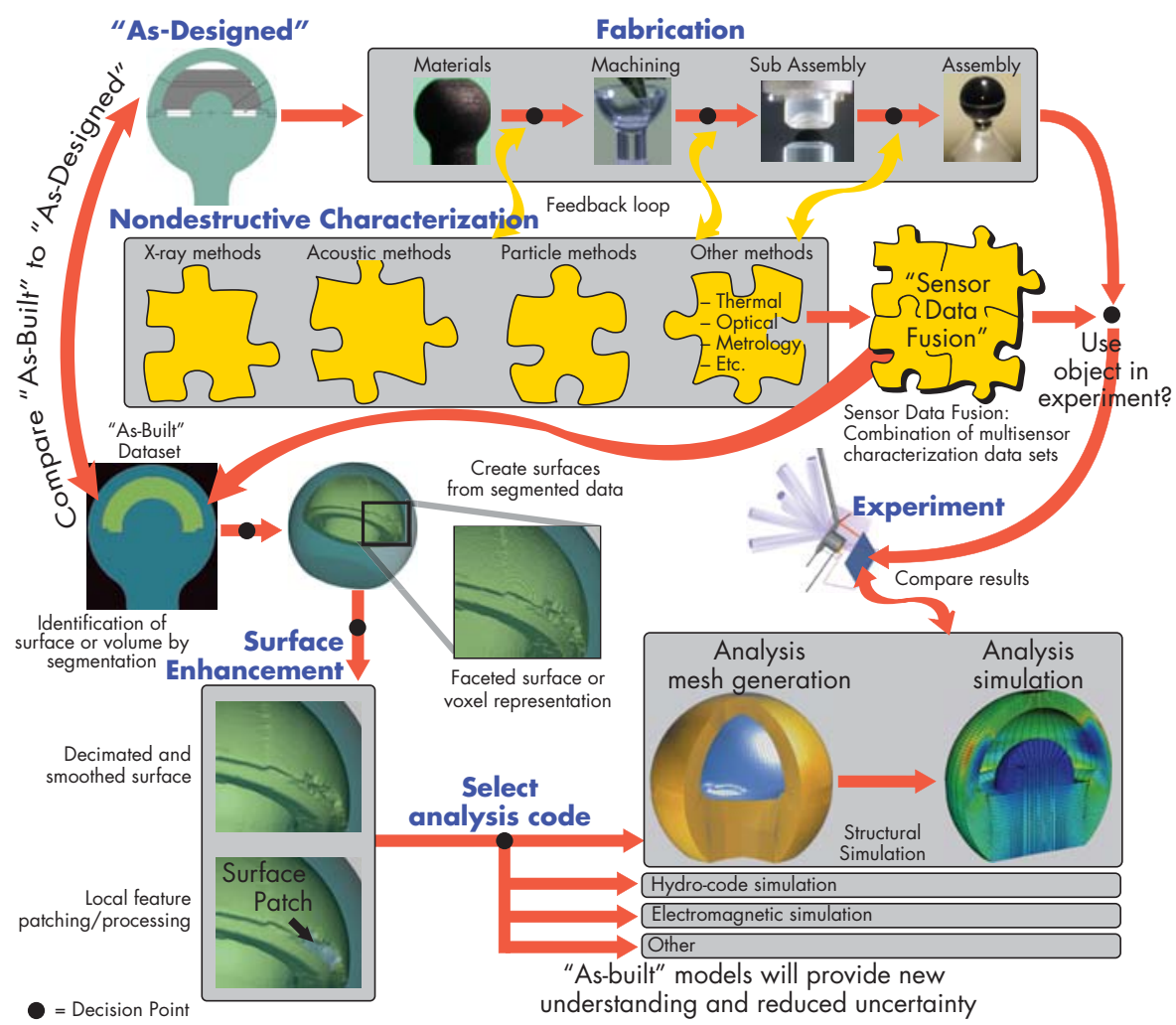

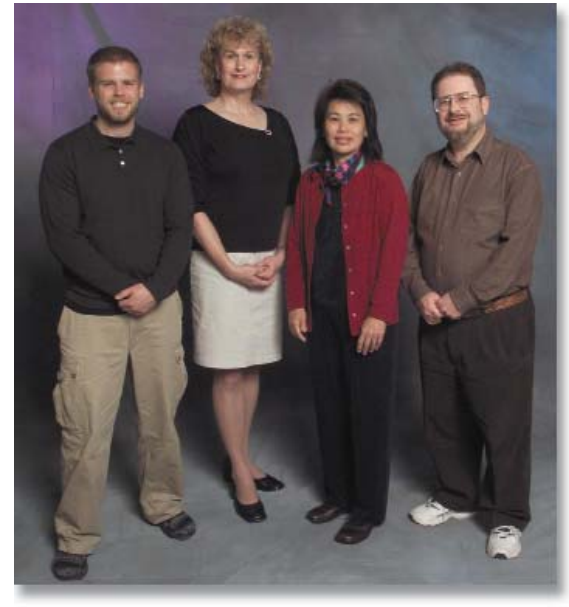

Project team (left to right): Edwin Kokko, Grace Clark, Diane Chinn, and Dave Chambers. Not pictured: Jessie Jackson.

Figure 2. As-built modeling concept applied to a NIF spherical reference standard target. 


\section{Neutron DR/CT Upgrade at McClellan Nuclear Radiation Center}

Figure 1. MNRC's Bay 4, showing detector mounting hardware, object handling table, and beam shutters.

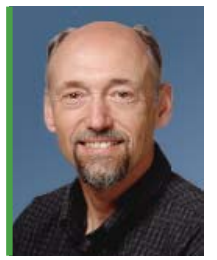

For more information contact Daniel J. Schneberk (925) 423-3531, schneberk1@|lnl.gov

MNRC, enabling inspections with spatial resolution in the 50- to $100-\mu \mathrm{m}$ range.

eutron imaging can be a particularly

useful nondestructive technique for specimens containing trace amounts of hydrogen. Consequently, neutron-based inspections are recommended for evaluating fluid flow in soil and rocks, and in the inspection of high explosives. The McClellan Nuclear Radiation Center (MNRC) includes a neutron inspection capability established by the U.S. Air Force. The University of California, Davis (UCD) is now overseeing MNRC, furthering the conversion of inspection bays to nonmilitary uses. In this project, LLNL is assisting in the digital radiographic and computed tomographic (DR/CT) upgrade (Fig. 1). The overall goal of the collaborative work is the construction of DR/CT capabilities at

\section{Project Goals}

A number of specific tasks were undertaken: 1) purchase new hardware for Bays 3 and 4; 2) install LLNL DR/CT software for acquiring, processing, reconstructing, and displaying DR/CT data; 3) collaborate with MNRC/UCD staff on the fielding of hardware; 4) perform detailed studies of scintillators; 5) perform DR/CT of soil samples and selected rock samples; 6) perform DR/CT of high explosive samples; 7) perform detailed comparisons between data from LLNL $\mathrm{x}$-ray systems and MNRC neutron scans; and 8) participate in focused exchanges with UCD professors and graduate students.

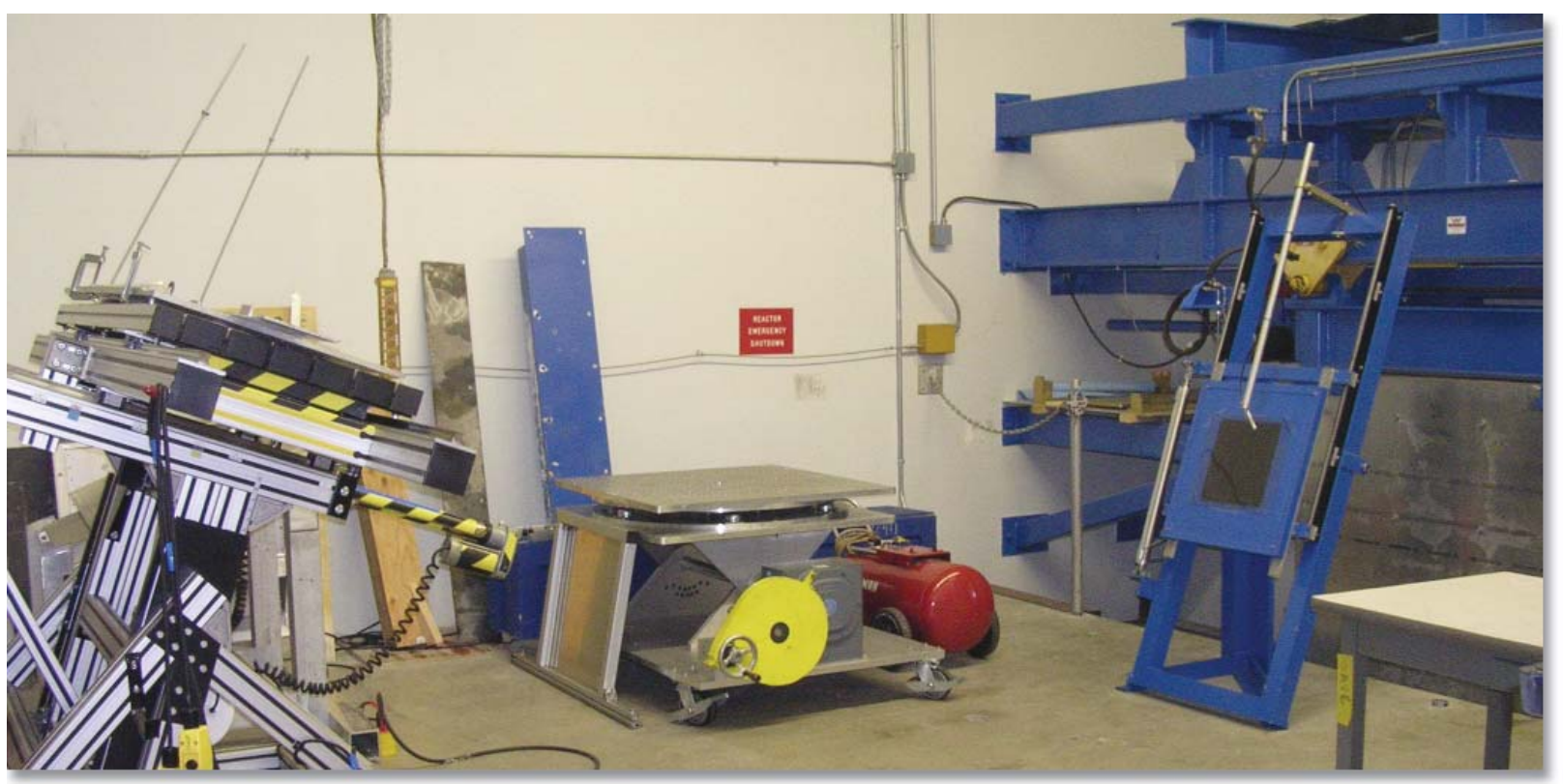




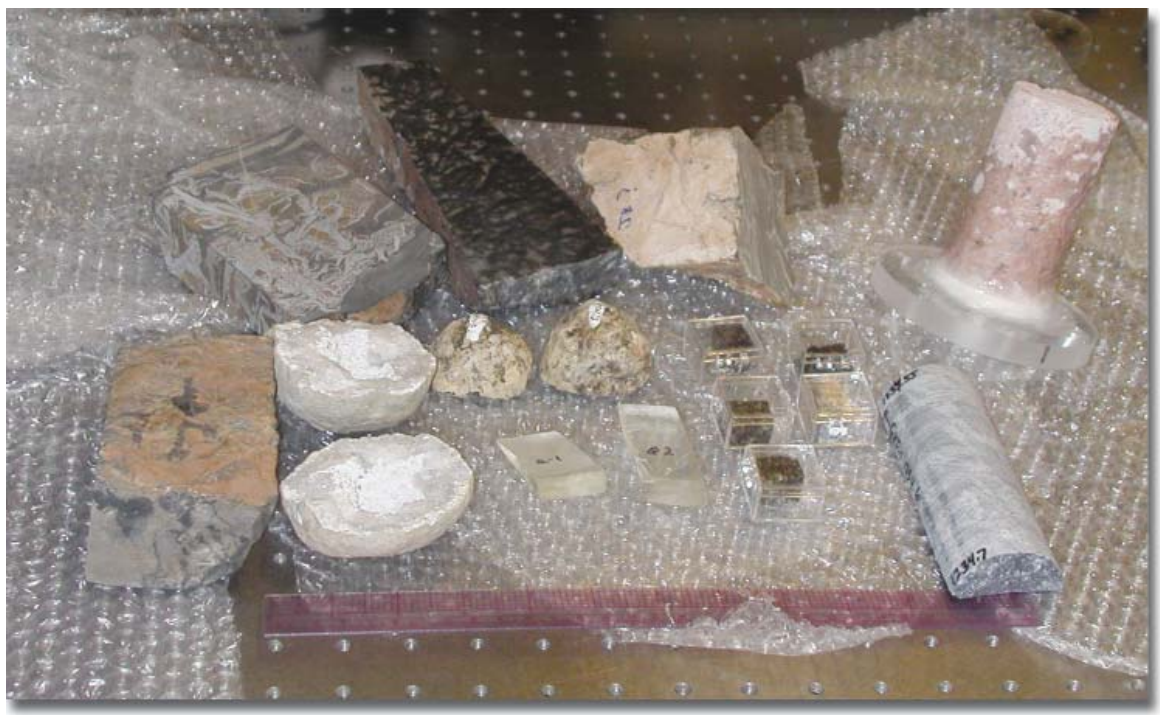

Figure 2. UCD objects scanned at LLNL using the x-ray CT scanner PCAT.

\section{Relevance to LLNL Mission}

Successful completion of this collaboration will provide robust access to a world-class neutron-based inspection facility. Scientists in energetic materials, environmental restoration, and earth sciences have applications for neutron-based inspections. Collaboration with UCD provides access to staff and technology at the Imaging Sciences Center, and an opportunity to familiarize them with LLNL projects and applications.

\section{FY2004 Accomplishments and Results}

All of the hardware for the Bay 3 and Bay 4 upgrades have been purchased by MNRC, and LLNL software is installed on data acquisition computers and bench-tested.

Due to staffing changes, the three tasks that involved data acquisition at MNRCthe scintillator evaluation, the scanning of soil samples, and scans of high explosives - could not be completed in FY2004, but a variety of UCD samples has been scanned (Fig. 2).

Generating this scan data at LLNL has three benefits. First, UCD researchers gain experience in manipulating $\mathrm{CT}$ volumes at the target spatial resolution for the scanner upgrade. Spatial resolution on LLNL's PCAT is about $100 \mu \mathrm{m}$, and current MNRC spatial resolution is about $500 \mu \mathrm{m}$ (Fig. 3). PCAT has the same type of detector (camera-scintillator) as that being installed at MNRC and has signal-to-noise similar to the data expected from MNRC.

Second, we will have completed the $\mathrm{x}$ ray scanning phase of this project, in preparation for the detailed comparison to neutron DR/CT results. Lastly, the scans provide a good venue for further collaboration in the coming year.

Several images from a series of volcanic cubes and an ignibrite sample were scanned by PCAT. These and other data are being delivered to UCD geology professors and staff at the Imaging Sciences Center.

Further UCD/LLNL collaborative work was pursued. Current MNRC scanned data was reconstructed and analyzed using LLNL software. Working copies of LLNL software are resident at UCD for CT processing, reconstruction, and analysis. A collaborative proposal, "Combining Neutron and X-Ray Computed Tomography for Nondestructive Imaging," was submitted for funding.

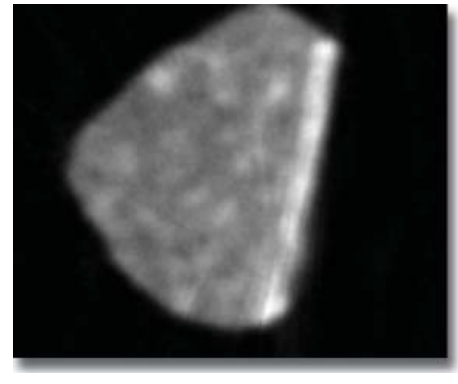

Figure 3. Neutron data from MNRC illustrating spatial resolution of currently fielded capability. Image is of a cross-sectional CT slice of a UCD rock sample (25 mm on the longest side).

\section{FY2005 Proposed Work}

An additional year of funding will enable us to complete the work started this year. Four tasks remain: finish the physical upgrades to Bay 3; field the new detectors; perform scintillator evaluation and system characterization; and scan rocks, soil and LLNL HE samples. 


\section{Quantitative Characterization of Mesoscale Objects}

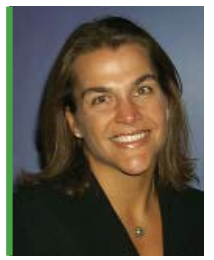

For more information contact Amy M. Waters (925) 423-2424, waters4@\|lnl.gov

\section{Project Goals}

Our project goal was to quantify the performance of mesoscale imaging systems. We chose four x-ray systems: two microfocus systems, KCAT (LLNL) and Xradia $\mu \mathrm{XCT}$ (Xradia, Inc., Concord, California); and two synchrotron-based systems, Beamline 1-ID at the Advanced Photon Source (APS) and the

Tomography Beamline at the Advanced Light Source (ALS).

\section{Relevance to LLNL Mission}

This project directly supports longterm plans to apply and understand mesoscale characterization techniques, and to build a facility to fabricate and characterize mesoscale target assemblies for studying high-energy-density physics (HEDP). Understanding and benchmarking the capabilities of these systems will enable the science and technology of phase-contrast modeling and object recovery. LLNL programs that would

Figure 2. Photograph of one large radius of curvature edge made of Cu coated with Au and used to measure 2-D MTF.

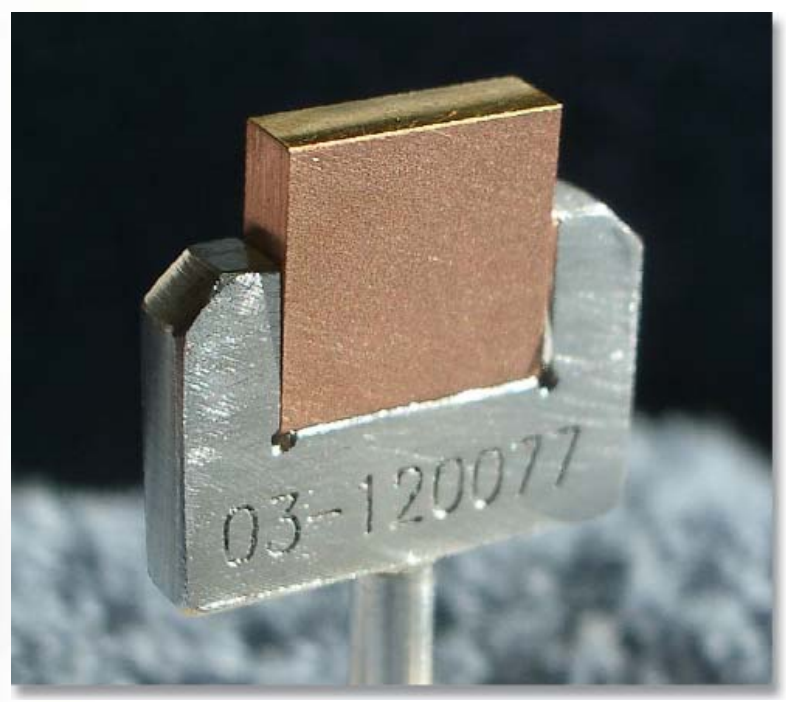


benefit from this project include HEDP and inertial confinement fusion experiments for NIF. This project is consistent with LLNL's plans to study the suitability of applying known engineering approaches to solve problems of interest.

\section{FY2004 Accomplishments and Results}

We have benchmarked the four x-ray mesoscale imaging systems described above, quantifying the 2-D DR performance by measuring the 2-D Modulation Transfer Function (MTF) and signal-tonoise ratio (SNR) using edge artifacts. First, a thin tantalum foil was used to measure MTF and SNR. MTF results for each of the four systems are shown in Fig. 1.

Due to the excessive raggedness of the edge on the tantalum foil, we fabricated eight serialized Au-plated $\mathrm{Cu}$ edges, shown in Fig. 2. It has previously been shown that for HEDP imaging, $x$ rays at 10 to $80 \mathrm{keV}$ are of interest. The new $\mathrm{Au}$-plated $\mathrm{Cu}$ edges provide a transmission of $1 \%$ for $60-\mathrm{keV}$ photons passing through the edge $1 \mu \mathrm{m}$ from the surface. Additional MTF and SNR measurements were made using the new edges and compared to the results for the tantalum foil.

We then imaged more complex objects such as the mesoscale spherical reference standards built in FY2003 (Fig. 3). DR and CT data was also acquired for the planar reference standard. The 2- and 3-D data from the mesoscale reference standards was used to create meshes for input to finite element analyses.
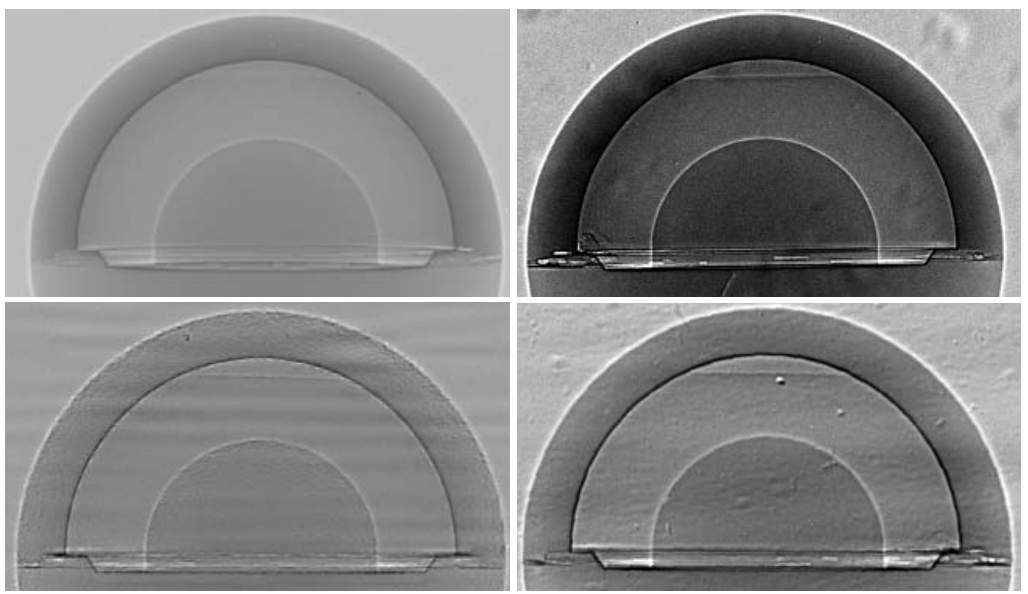

Figure 3. Transmission images of spherical mesoscale reference standard acquired with four x-ray systems. Top left: KCAT, top right: Xradia, bottom left: ALS, bottom right: Beamline 1-ID APS.

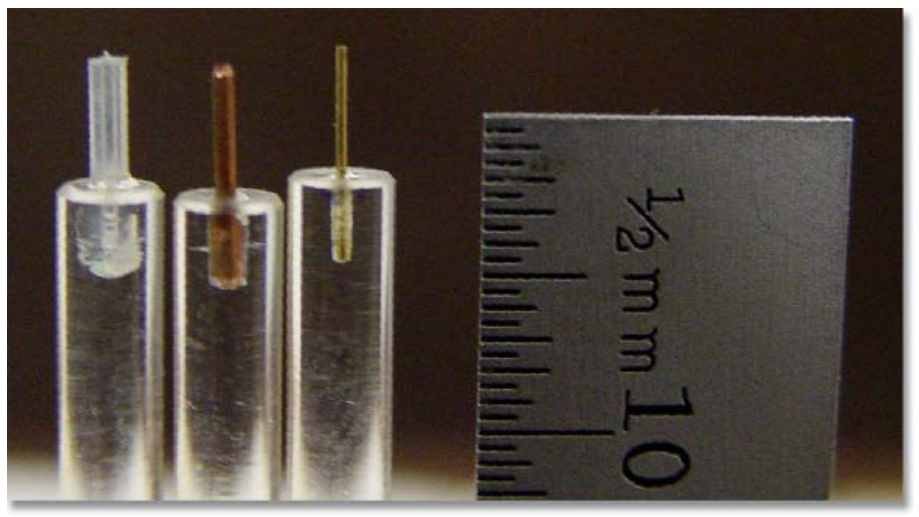

Figure 4. Photograph of tubes used to measure 3-D MTF. From left: low-density polyethylene (LDPE), Cu and Au. 


\section{Three-Dimensional Rendering of High-Frequency Ultrasonic Data}

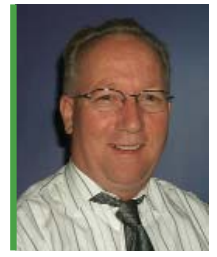

For more information contact Steven E. Benson (925) 422-7024, benson4@\|lnl.gov

rendering of ultrasonic images greatly increases our ability to interpret the data. Our customers gain a better understanding of the results when viewed in 3-D. Also, our ability to characterize components in 3-D will support as-built modeling of the sub- and full assemblies.

The result of this project is a computer workstation capable of processing and rendering complex 3-D ultrasonic data sets. This new tool evaluates engineering materials in 3-D using ultrasonic imaging techniques and provides volumetric information.

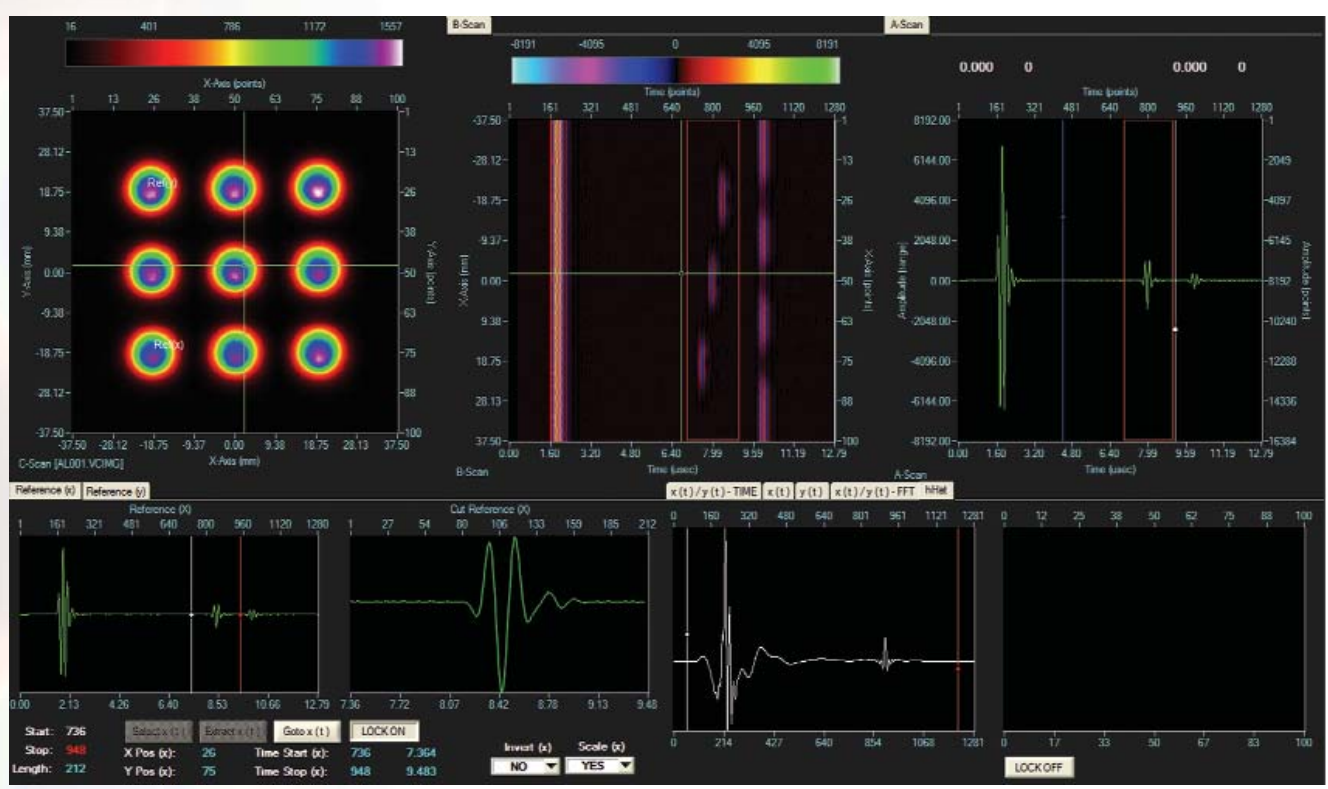

Figure 1. Screen capture of LabWindows CV-MATLAB user interface. The user is able to view 3-D volume data sets and set-processing variables. 

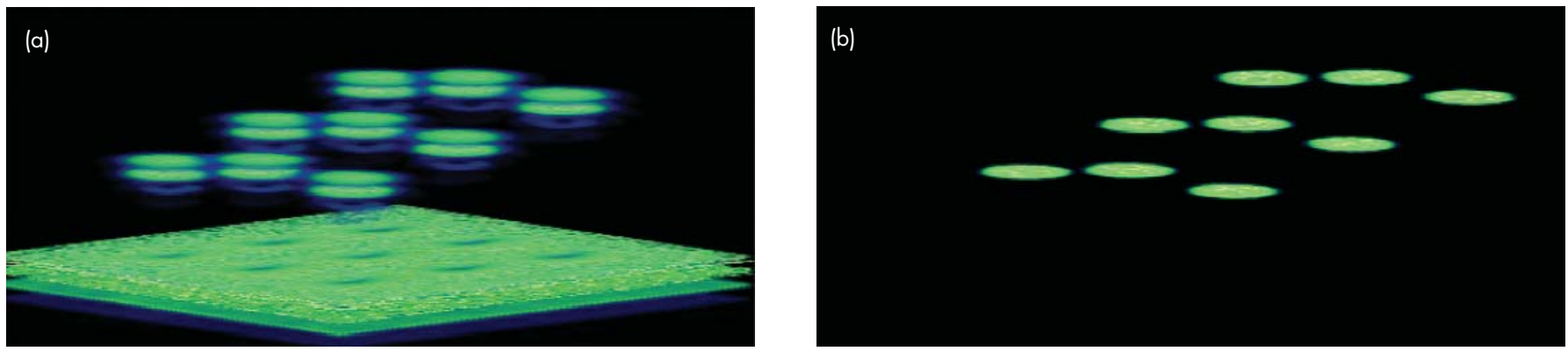

Figure 2. Results of (a) unprocessed (with temporal artiffact), and (b) processed (without temoral artifact), 3-D ultrasonic data set acquired on a set of flat bottom holes machined in a test phantom. The holes are 6-mm diameter and located at depths that range from 4.5 to $7.5 \mathrm{~mm}$ at 12.7 -mm spacing.

\section{Project Goals}

There were three main goals for FY2004: provide a MATLAB signal processing algorithm to remove or minimize the transducer ringing on 1-D time domain signals; provide a computer user interface around the MATLAB code that is a comprehensive utility for interactively processing large 3-D ultrasonic data sets; deploy a computer workstation optimized to process and interactively view and manipulate volumetric 3-D ultrasonic data sets.

\section{Relevance to LLNL Mission}

This ultrasonic volumetric imaging tool will provide quantitative information on engineering materials across many Laboratory programs including NIF, Weapons, and surveillance activities.

\section{FY2004 Accomplishments and Results}

Algorithms. We generated a signal processing algorithm and MATLAB software for spatio-temporal resolution enhancement of ultrasonic NDE signals. Given a measured reflection signal and an associated reference signal, the algorithms preprocess the signals (e. g., remove mean, low-pass filter, decimate); produce an optimal least-squares estimate of the impulse response of the material under test; and post-process the signals (e. g., filter, interpolate). The estimated impulse response, when used in place of the raw reflection signal, enhances the spatial resolution of the ultrasonic measurements by removing distortion caused by the limitedbandwidth transducers and the materials under test.

Computer user interface. The MATLAB code processes single 1-D time domain signals and was not optimized to process large 3-D ultrasonic data sets containing as many as 250,000 1-D time domain signals. A user interface was needed to provide a utility to read in 3-D volume data sets and to optimize signal-processing variables.

LabWindows CVI (C for Visual Instruments) was used to create the user interface (Fig. 1). The user interface serves three purposes: it is a utility to load and interactively view 3-D ultrasonic data sets; it optimizes MATLAB signal processing input variables; and, using MATLAB engine, it provides a Dynamic Link Library between the user interface code and the MATLAB program environment.
Computer workstation for 3-D rendering. Combining the work performed this year on hardware and software, a computer workstation was deployed. The workstation provides a stable platform for engineers and technicians to process and display 3-D ultrasonic data sets in real time, adding insight to the materials inspected and useful volumetric information. Figure 2 shows the results of unprocessed (with temporal artifact) and processed (without temporal artifact) 3-D ultrasonic data sets acquired on a set of flat bottom holes machined in a test phantom.

\section{Related Reference}

Clark, G. A., D. M. Tilly, and W. D. Cook, "Ultrasonic Signal/Image Restoration for Quantitative NDE," NDT International, 19, (3), June 1986. 


\section{Ultrasonic Calibration Test Phantom}

(a)

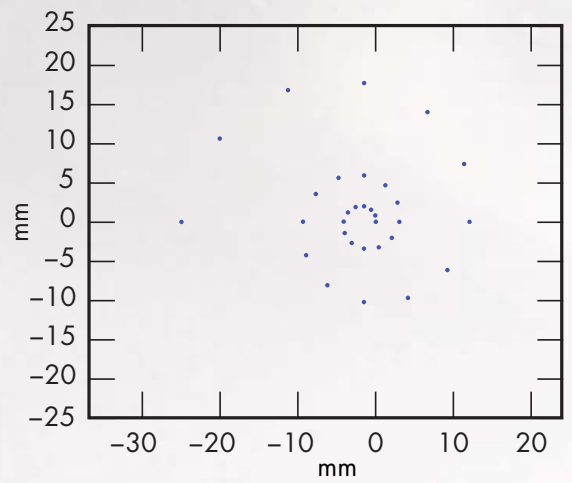

(b)

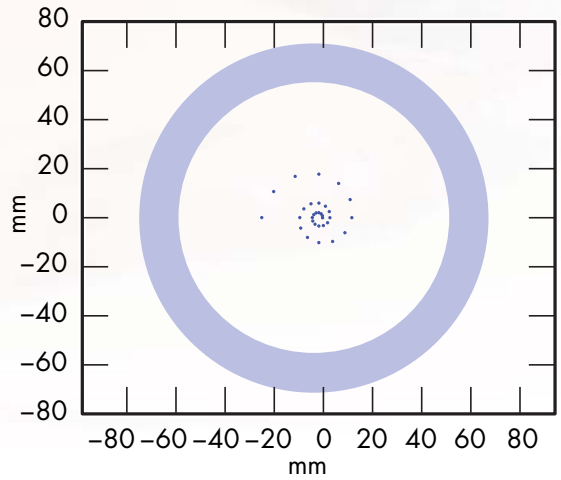

$W^{\circ}$ e built a phantom, the Ultrasonic Calibration Test Phantom, consisting of vertical wires maintained under tension. The phantom is a valuable tool as an ultrasonic test, calibration, and reconstruction object for the LLNL annular array scanner.

\section{Project Goals}

Our goal was to build a reconfigurable ultrasonic phantom for the annular array scanner. Its intended use was to collect well-characterized data under controlled conditions for use as "canonical data sets" in testing and evaluating new inversion algorithms.

\section{Relevance to LLNL Mission}

The nondestructive evaluation (NDE) of objects and media of interest to LLNL, DOE, and DoD is an essential part of LLNL's mission. Many NDE situations have put demands beyond the scope of the current suite of imaging algorithms. As

(c)

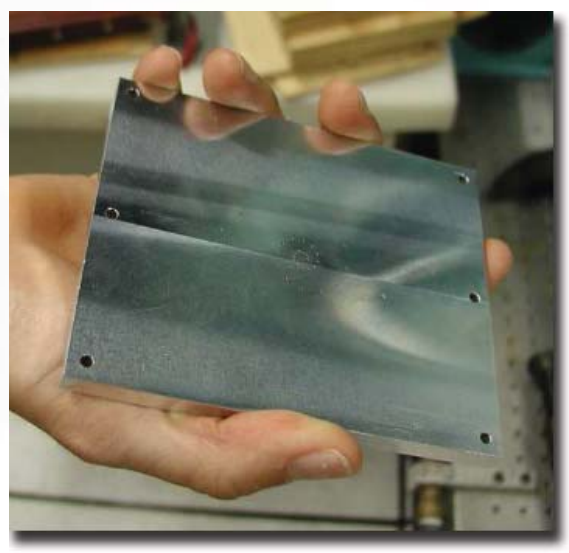

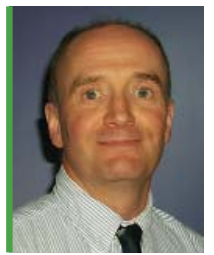

For more information contact Sean K. Lehman (925) 423-3580, lehman2@|ln..gov

new algorithms and codes come on line, they must be evaluated on well-characterized phantoms. The Ultrasonic Calibration Test Phantom provides such a data set.

\section{FY2004 Accomplishments and Results}

We achieved our goal of building the Ultrasonic Calibration Test Phantom. The phantom consists of top and bottom plates into which are drilled holes through which wires are run. The holes form a geometrical pattern that governs the horizontal distribution of the wires. The plates are affixed to top and bottom mounting brackets that are pulled apart, putting the wires under tension. Given that the phantom object formed by the wire distribution does not vary much in the vertical $(\mathrm{z})$ direction, we assume the measured data are from a 2.5-D object.

These plates are removable, permitting different configurations to be used. We currently have only one configuration: a logarithmic spiral with equation,

$$
r\left(\theta_{n}\right)=\alpha\left(\cos \left(\theta_{n}\right), \sin \left(\theta_{n}\right)\right) e^{b \theta_{n}},
$$
where

$\alpha \equiv \lambda_{0} \quad$ is the initial radius and, $\lambda_{0} \equiv v_{0} / f_{0}$ is the insonifying wavelength,

$v_{0} \equiv 1500 \mathrm{~m} / \mathrm{s} \quad$ is the assumed background water velocity,

$\mathrm{f}_{0} \equiv 1 \mathrm{MHz}$ is the approximate insonifying frequency,

$\mathrm{b} \equiv 10 \pi / 180 \quad$ is the spiral growth rate, $\theta_{\mathrm{n}} \equiv\{\mathrm{n} \Delta \theta\}_{\mathrm{n}=0}^{\mathrm{N}-1}$ are the angular locations of the wires,

$\Delta \theta \equiv 30 \pi / 180$ is the angular increment, $\mathrm{N} \equiv 31$ is the number of wires.

Figure 1. Basic spiral phantom design (a) without hollow cylinder, (b) with cylinder. (c) Photograph of wiring plate with holes. 
(a)

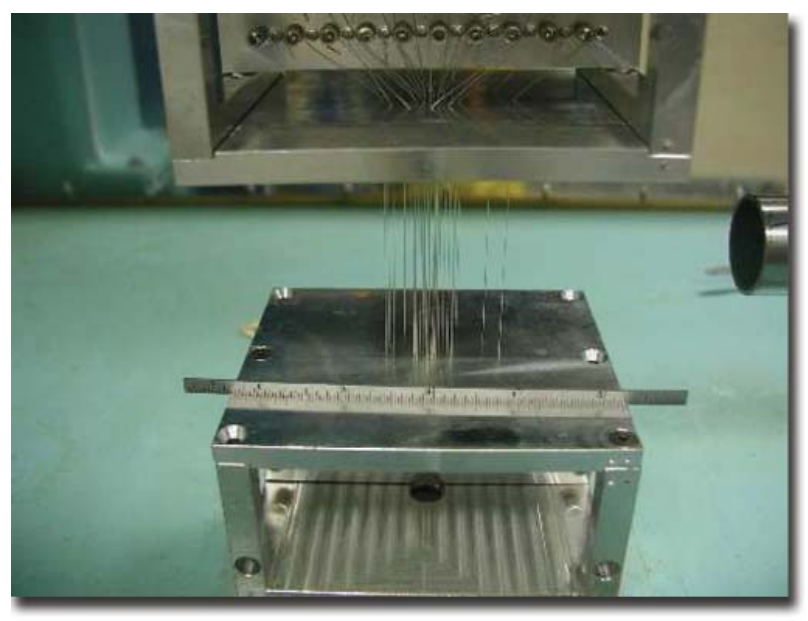

(b)

Figure 2. (a) Phantom after the wires were pulled taut. (b) Phantom with optional acrylic hollow cylinder.

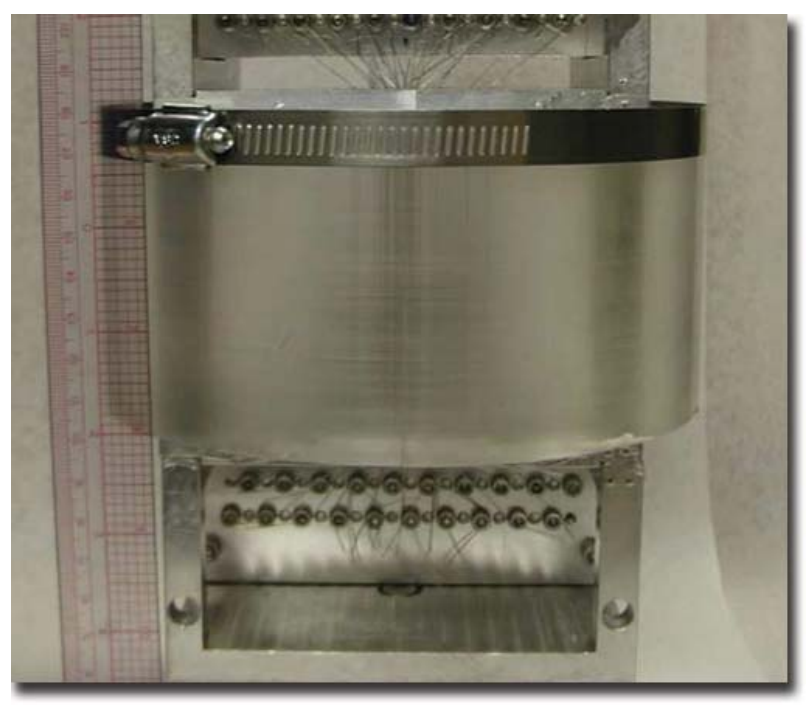

Our current configuration is shown in Fig. 1. We have allowed for an optional acrylic hollow cylinder around the phantom as shown in Fig. 1b. Figure 1c shows one of the plates.

The phantom is secured into the scanner: the top block is affixed to a rod fastened to the central bore of the scanner drive; the bottom block is fastened to the bottom of the tank. Once installed, the blocks are pulled apart to render the wires taut. Figure 2a shows the phantom with 31 steel taut wires; Fig. 2b shows the optional acrylic cylinder in place.

We have run scans with the following combinations of materials:

- 31 steel wires

- 30 steel and one nylon wire

- 25 steel and six nylon wires

- 25 steel, and six nylon with a hollow cylinder
- two steel, two nylon resolution pair

- two steel, two nylon resolution pair with a hollow cylinder

- a hollow cylinder only (no wires)

- water only background run.

\section{Related References}

1. Lehman, S. K., and A. J. Devaney, "Transmission Mode Time-Reversal Super-Resolution Imaging,"

Journal of the Acoustical Society of America, 113,

(5), pp. 2742-2753, May 2003.

2. Devaney, A. J., and M. Dennison, "Inverse Scattering in Inhomogeneous Background Media," Inverse Problems, 19, pp. 855-870, 2003.

3. Lehman, S. K., and S. J. Norton, "Radical Reflection Diffraction Tomography," Journal of the Acoustical Society of America, 116, (4), October 2004.

\section{FY2005 Proposed Work}

We are very pleased with the quality of the measured data, which will be used as "canonical data sets" for testing new inversion and detection algorithms. The flexibility of the phantom allows us to create new models to simulate real NDE problems of interest to LLNL, DOE, and DoD. Additionally, we will share these data sets with outside collaborators, such as those at the Center for Subsurface Sensing and Imaging Systems, to identify NDE solutions of interest to LLNL.

We will be reconstructing the current data sets with our three current, mature algorithms: time reversal, Hilbert space inverse wave, and quantitative timedomain multiview imaging. 

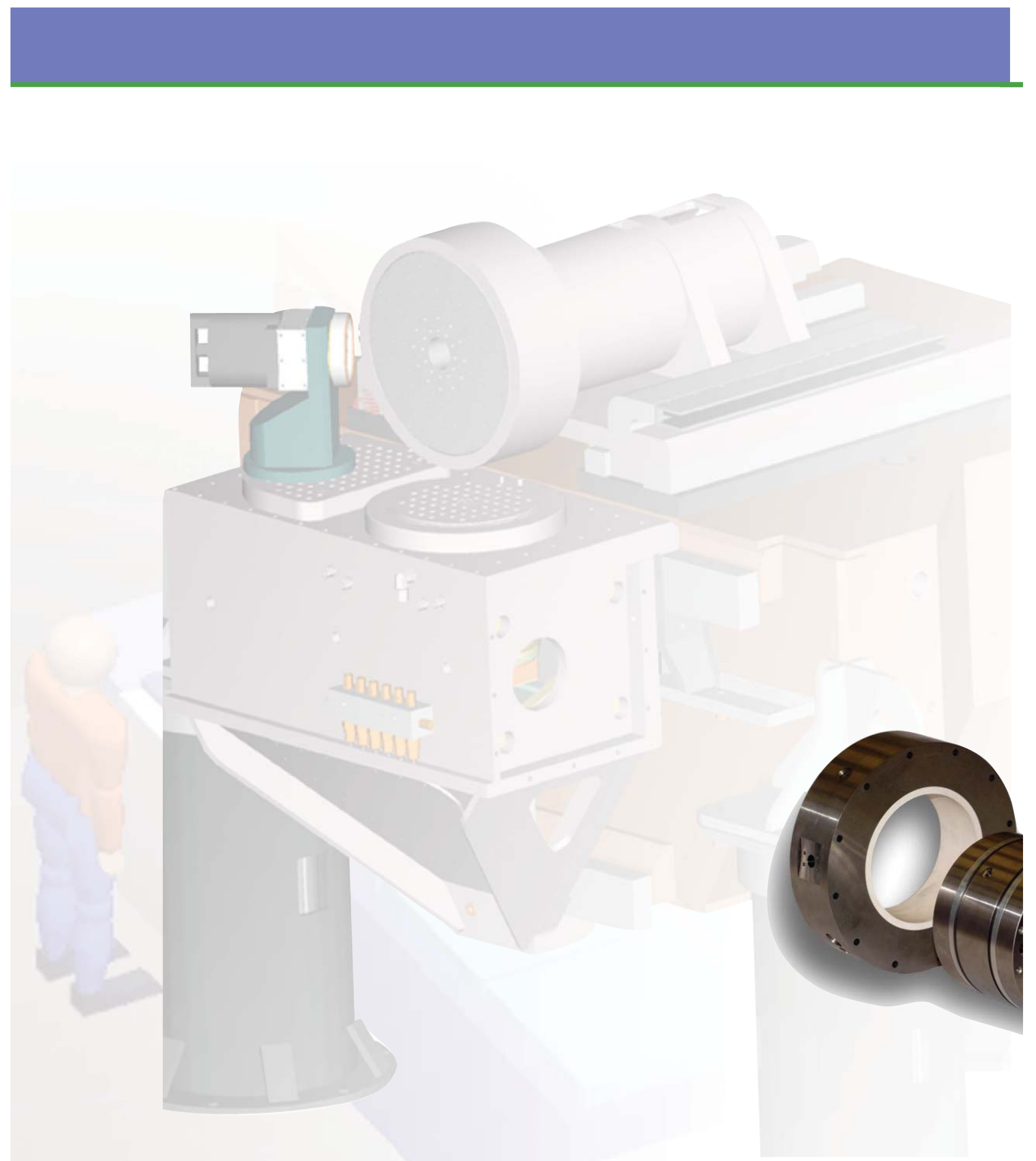

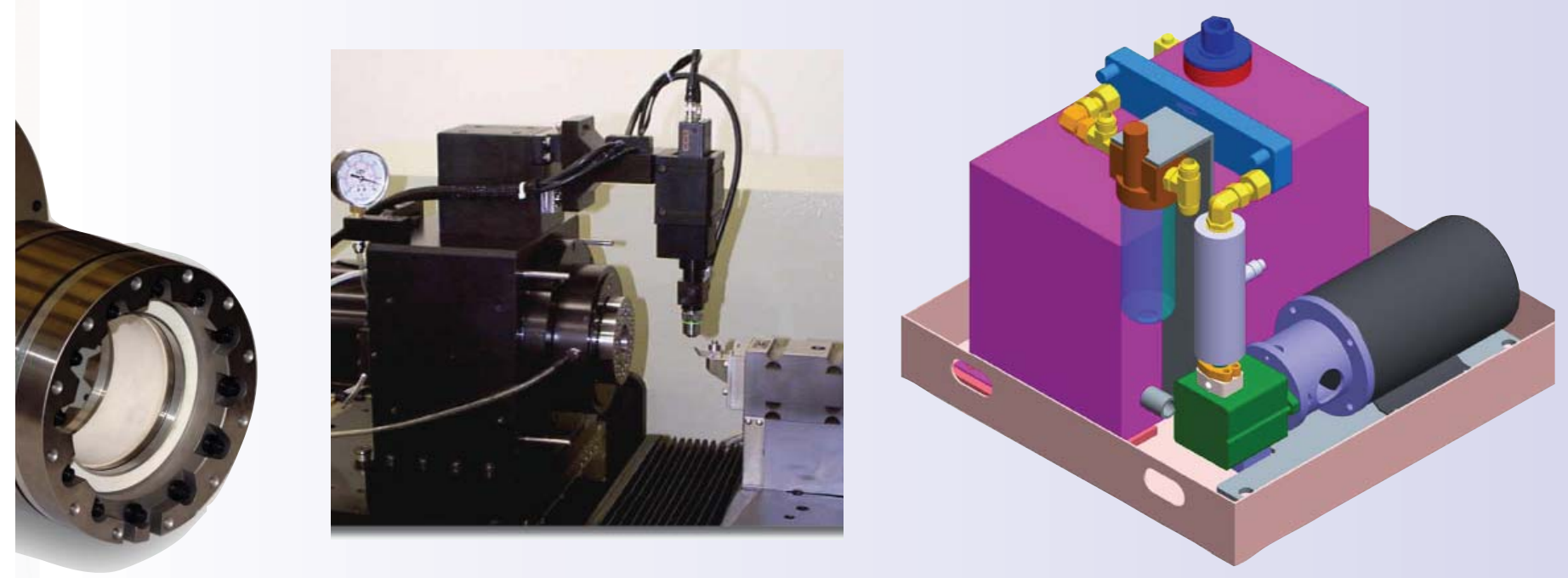

\section{Center for \\ Precision Engineering}

\section{TechBase}




\section{Hydraulic Power Unit for Hydrostatic Bearings}

Figure 1. Schematic of system.

(The regulator was not used).

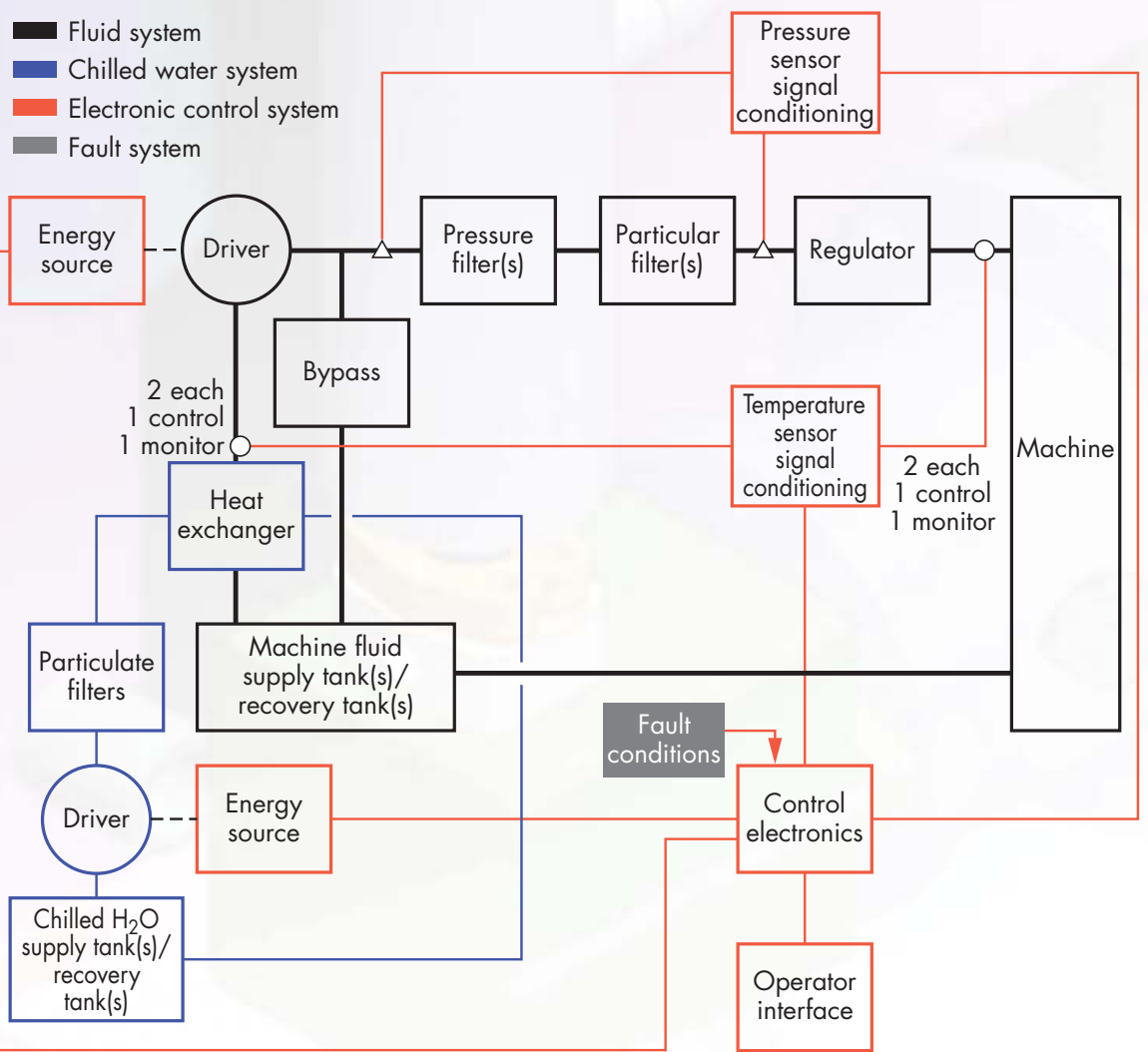

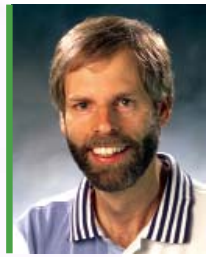

For more information contact David J. Hopkins (925) 423-6134, hopkins3@|lnl.gov

degree of stable pressure and temperature control. Key factors addressed are the refinement of computer models that aid in the construction of hydrostatic fluid pressure and temperature control systems.

\section{Project Goals}

Repeatability is an important characteristic of a precision machine tool. By investigating the sources of non-repeatability, it is possible to quantify and control these sources. A machine expands and contracts with temperature. A new LLNL machine, the Precision Optical Grinder and Lathe (POGAL) has an approximately $350-x-$ 350-mm work volume and an accuracy of $10 \mathrm{~nm}$ RMS. The temperature of this machine must be held to mili-degree levels to maintain machine repeatability within acceptable limits.

Pressure control is also important. A machine axis will move due to oil pressure fluctuations of the slide bearings. Many older LLNL precision machines use an airover-oil dual accumulator control system. The pressure fluctuations in this type of system are low, but can be problematic when the system switches from the empty accumulator to the full accumulator as a pressure spike momentarily occurs.

The project goals were to model and build a highly stable and integrated pressure and temperature control system for use on precision machines. The model helped size components for two systems covering a range of hydrostatic bearing requirements. In addition, the model is available for sizing any new system.

\section{Relevance to LLNL Mission}

LLNL has a long history of contributions to the field of precision engineering. 
Some of our machines support target and fixture fabrication for NIF and weapons programs. They can fabricate precision optics by diamond turning. The continuing investment in the future of machines such as the POGAL will help the Laboratory be prepared for the expanding field of x-ray optics. This project supports the ability to build new precision machines and to modernize older ones.

\section{FY2004 Accomplishments and Results}

Figures 1 to 3 illustrate our system. In FY2004, models have been fine-tuned for the pressure control system of the hydrostatic bearings and for the heat exchanger characteristics over a range of fluid flowrates and viscosities. Two pressure and temperature control systems cover a range of precision machine needs, from the low-heat-load requirements of the slide bearings to the dynamic higher-heat-load demands of a spindle bearing.

The electronic control system looks industrialized, but is actually a sophisticated dual-loop control system capable of temperature and oil pressure control. The system works to maintain pressure by using a variable frequency drive motor/pump in a PID loop to control pressure. Temperature control is done in a similar manner with dual PID control loops, using either a variable frequency drive motor/pump or a proportional valve to vary the flow rate of chilled water through the heat exchanger. An operator interface to the PLC has been created via LabView, allowing temperature and pressure data to be observed and trended. In addition, LabView provides a data communication interface to the machine tool controller. Modernized high-stability thermistor signal conditioning electronics for temperature sensing has been completed.

The system includes a specially selected pump that minimizes pressure fluctuations, and an accumulator/restrictor combination similar to an electronic low-pass filter that further reduces any other pressure variations. All system parts have arrived on site and we plan to complete the assembly of both systems.

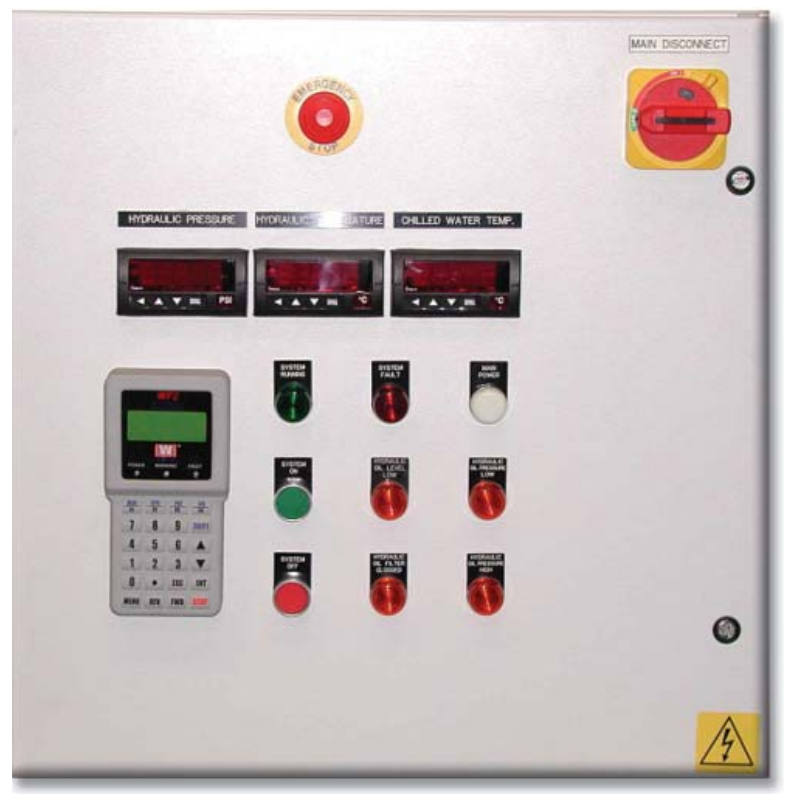

Figure 2. Electrical/electronic control panel.

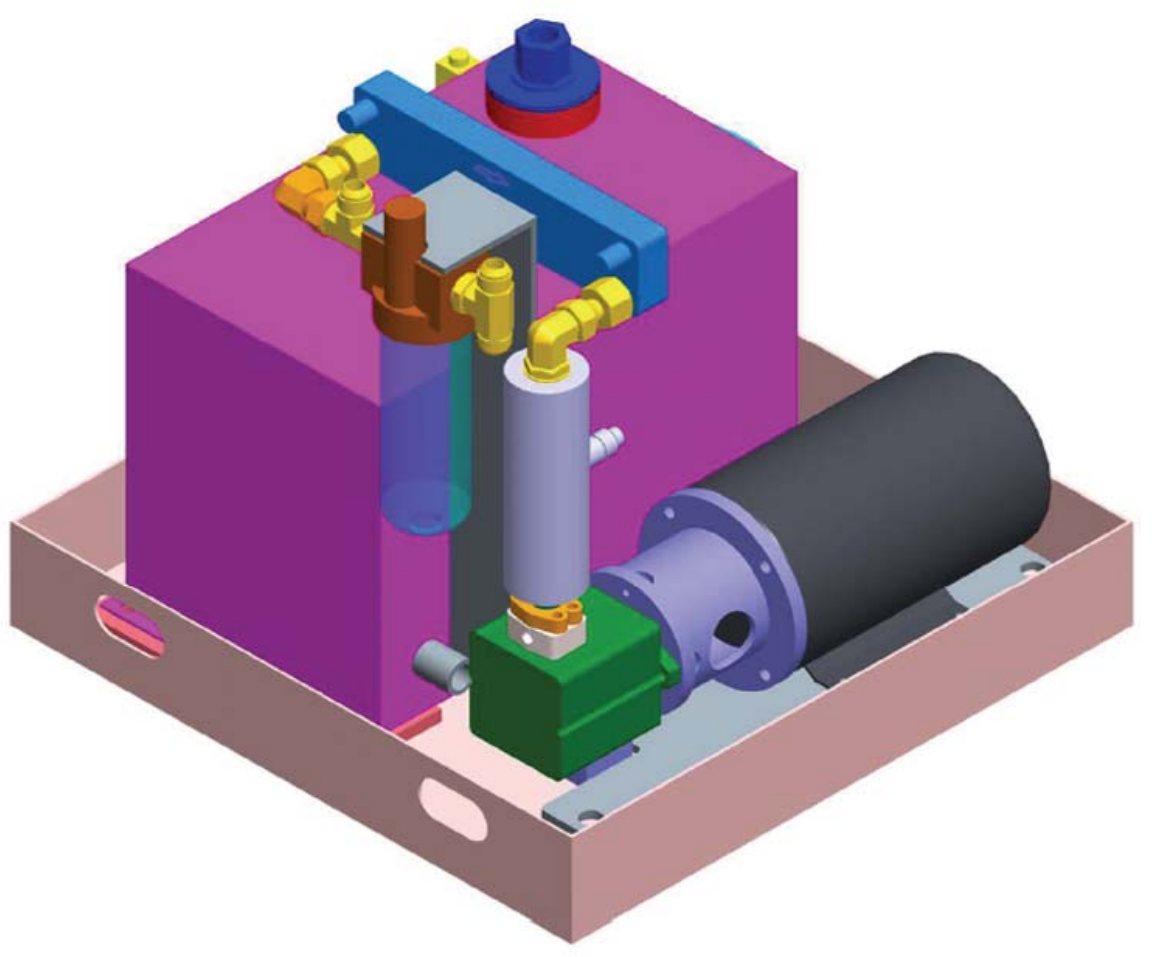

Figure 3. Hydraulic system mechanical layout. 


\section{Noncontact Diamond Tool Metrology}

the fabrication of high-energy-density tools with small nose radii and high clearance angles (making a "sharper" tool) that are extremely fragile. The tool geometry is driven by the need to access features with very small spatial wavelengths (high-frequency sine waves) or small radii, such as small hemispherical shapes. The LLNL standard approach to tool setting is an air-bearing LVDT, but with these fragile tools, there is risk using a contact method. Identifying noncontact diamond tool measurement technology would allow LLNL to minimize the risk of damage.

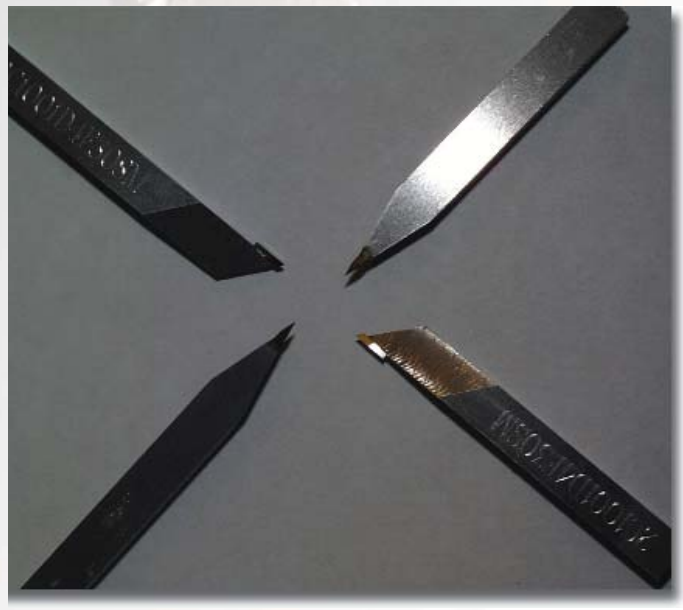

Figure 1. Typical diamond tool geometry for HEDP target fabrication, 1/4- in. shank.

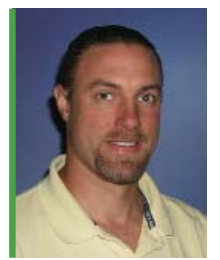

For more information contact Jeremy J. Kroll (925) 422-6437, kroll4@\|nl.gov

\section{Project Goals}

The goal of this project is to evaluate the current state of the art for measuring diamond tool position, non-roundness, and nominal radius, using noncontact methods.

\section{Relevance to LLNL Mission}

Commercial diamond turning machines (DTMs) come equipped with a microscope/digital-camera-type tool set station that is accurate to only a few microns. A modest improvement in the commercial noncontact tool setting system will allow higher efficiency in the fabrication of targets. If the resulting performance is better than that of air-bearing LVDTs, both in higher accuracy and improved spatial resolution, the system could be applicable to many of the precision machining and inspection applications at LLNL.

\section{FY2004 Accomplishments and Results}

Figure 1 shows a typical diamond tool geometry. To determine tool metrology requirements, three distinct classes of machines are considered: commercial machines from Precitech and Nanotechnology Systems with approximately 100-nm accuracy and 10-nm resolution; state-of-the-art machines such as LLNL's LODTM with 25-nm accuracy and 2.5-nm resolution; and next generation machines with 10-nm accuracy and 1-nm resolution. Using these machine specifications, resolutions and accuracies 


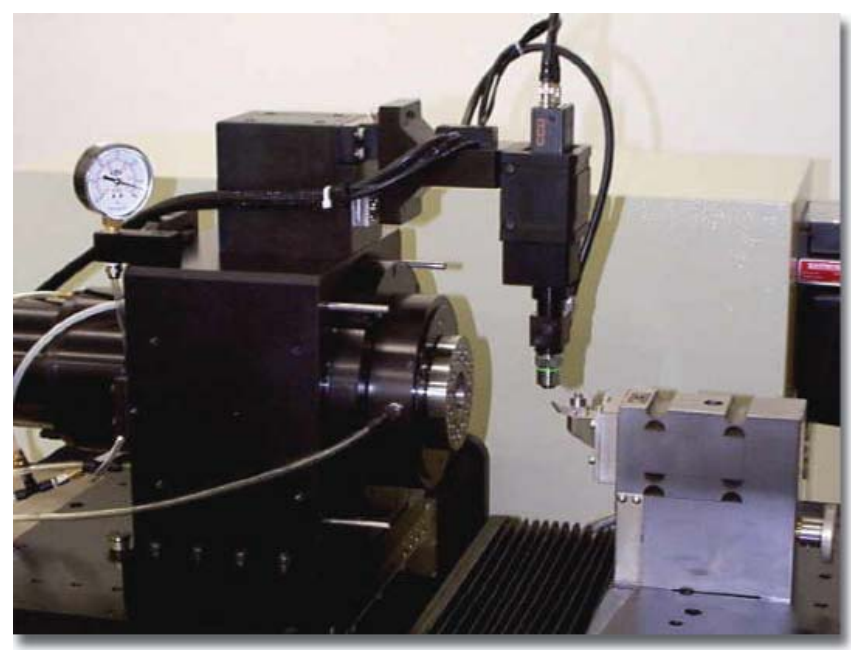

Figure 2. Commercial optical diamond toolset station from Moore Nanotechnology Systems. for tool parameters are determined. Profile or non-roundness requires the highest resolution because of the desire to capture high spatial frequency edge deviations. Size and position can be determined using lower resolution systems by averaging over the entire edge and curve fitting. Optical microscopy could be used for size and position, but would be completely inadequate for profile measurement.

Figure 2 shows a sample commercial optical diamond toolset station. Target fabrication uses DTMs with two different commercial toolset stations. Both of these stations suffer from the same shortcomings for precision manufacturing. These systems rely on the operator's selecting points on the tool edge from a display of the tool image. This is heavily dependent on not only the operator's qualitative judgment, but also the quality of the display. These cameras are analog output cameras, which can result in lower picture quality, and their optical subsystems are relatively low, fixed magnification. They are for much larger tools than are currently used in target fabrication. These systems could be improved with higher resolution cameras and higher magnification optical systems. Edge detection software could also enhance the toolset station size and position capability to the sub-micrometer level.

For profile measurement, an instrument with nanometer level resolution is required. One commercial instrument has been identified which claims to allow optical measurement of tool profile to nanometer level. The NanoInSPEC Edge Scanner can be purchased with 1-nm lateral position resolution. Although this system is expensive, it could be used to profile tools off the machine, while cheaper toolset stations are then used to locate the tool within the machine work volume.

For current and next generation machine tools, noncontact diamond tool metrology will be possible. This project determined that tool profiling requires nanometer resolution capability, while size and position can be obtained through lower resolution techniques using averaging and curve fitting.

\section{Related References}

1. Soares, S., "Nanometer Edge and Surface Imaging Using Optical Scatter” Precision Engineering, 27, pp. 99-102, 2003.

2. Morantz, P., "A Nanometric Precision Noncontact Toolsetting System," Proceedings of the 7th ASPE Conference, pp. 18-21, 1992.

3. Zhou, W., and C. Lilong, "Method for Edge Detection Based on Phase Jump in a Differential Interferometer," Applied Optics, 38 (1), pp. 152-159, 1999.

\section{FY2005 Proposed Work}

The next step should be to upgrade current DTM toolset stations and verify that they meet size and position measurement requirements. A commercial system is available which may meet our requirements for noncontact profile measurement. This instrument should be evaluated and, if accurate, purchased for diamond tool profiling. 


\section{Ultra-Precision Machine Spindle Using Porous Ceramic Bearings}

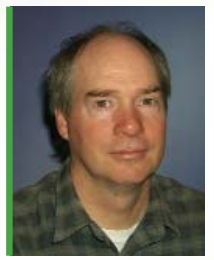

For more information contact Paul Geraghty

(925) 423-7351, geraghtyl@|ln.gov

A

bearing spindles have typically been

used for ultra-precision machine applications, due to the low asynchronous error motion and the high rotational accuracies achievable. Traditional oil-fluid-film bearings have not been able to match the accuracies of air bearings, but they have the advantage higher stiffness and improved dampening capabilities. With the advent of new oil-fluid-film porous ceramic bearing materials, we may now be able to have the best of both worlds: the accuracy of an air bearing with the high stiffness and improved dampening capabilities of a traditional oil-fluid-film bearing.

\section{Project Goals}

The main project goal is to have a working spindle, applying porous ceramic

bearing technology, which can be used on a future machine tool. The spindle will be thoroughly tested to ensure that it meets the performance requirements shown in the table.

Another goal of the project is to transfer the porous ceramic bearing technology from the academic field to LLNL, i. e., to create spindle modeling software tools and to increase LLNL's level of understanding of issues in building porous ceramic bearing spindles.

\section{Relevance to LLNL Mission}

Single-point diamond turning of optics was pioneered at LLNL, which received an R\&D 100 award for this work in 1978. Since that time, the sizes of the optics have become larger and the specifications

\begin{tabular}{|l|l|}
\hline \multicolumn{1}{|c}{ Work Head Performance Requirements } \\
\hline Speed & $100 \mathrm{to} 1,500 \mathrm{rpm}$, bi-directional \\
Load Capacity at Spindle Nose & $500 \mathrm{lb} .(227 \mathrm{~kg})$ including work holding device \\
\hline Error Motion: Axial & $2 \mu \mathrm{in}(50 \mathrm{~nm})$ \\
$\quad$ Radial & $2 \mu \mathrm{in}(50 \mathrm{~nm})$ \\
Static Stiffness: Axial & $720 \mathrm{~N} / \mu \mathrm{m}$ \\
& $300 \mathrm{~N} / \mu \mathrm{m}$ \\
\hline
\end{tabular}


have grown more stringent. For example, in 1978 we were fabricating 5 -cm optics, today we are fabricating optics that are $42 \mathrm{~cm}^{2}$, and have a surface finish requirement of under $1.5 \mathrm{~nm}$ RMS and a transmitted wavefront of less than $\lambda / 2$.

The work of this project will enable LLNL to continue its leadership in this field (see Fig. 1).

\section{FY2004 Accomplishments and Results}

This is a multiyear project that will be completed next year. Previous work included such tasks as: spindle and bearing models; fabrication of miscellaneous spindle and test hardware components; and fabrication, to a rough state, of the bearings and spindle rotor. This year we completed the fabrication of ceramic components; bearing gap analysis, based on actual ceramic bearing porosity; final precision machining of spindle bearings and rotor; and spindle thermal response modeling.

The optimum bearing porosity, determined by computational modeling of the ceramic bearings, was found to be $2 \times$ $10^{-14} \mathrm{~m}^{2}$. The measured porosity of the actual ceramic was $1.92 \times 10^{-14}$ and $1.72 \times 10^{-14} \mathrm{~m}^{2}$ for the front and rear journal bearings, respectively (Fig. 2). The actual porosity values were then used in our spindle model to determine the other bearing parameters required to complete the final precision machining of spindle bearings and rotor. This work was completed and we received the spindle components ready for assembly and testing.

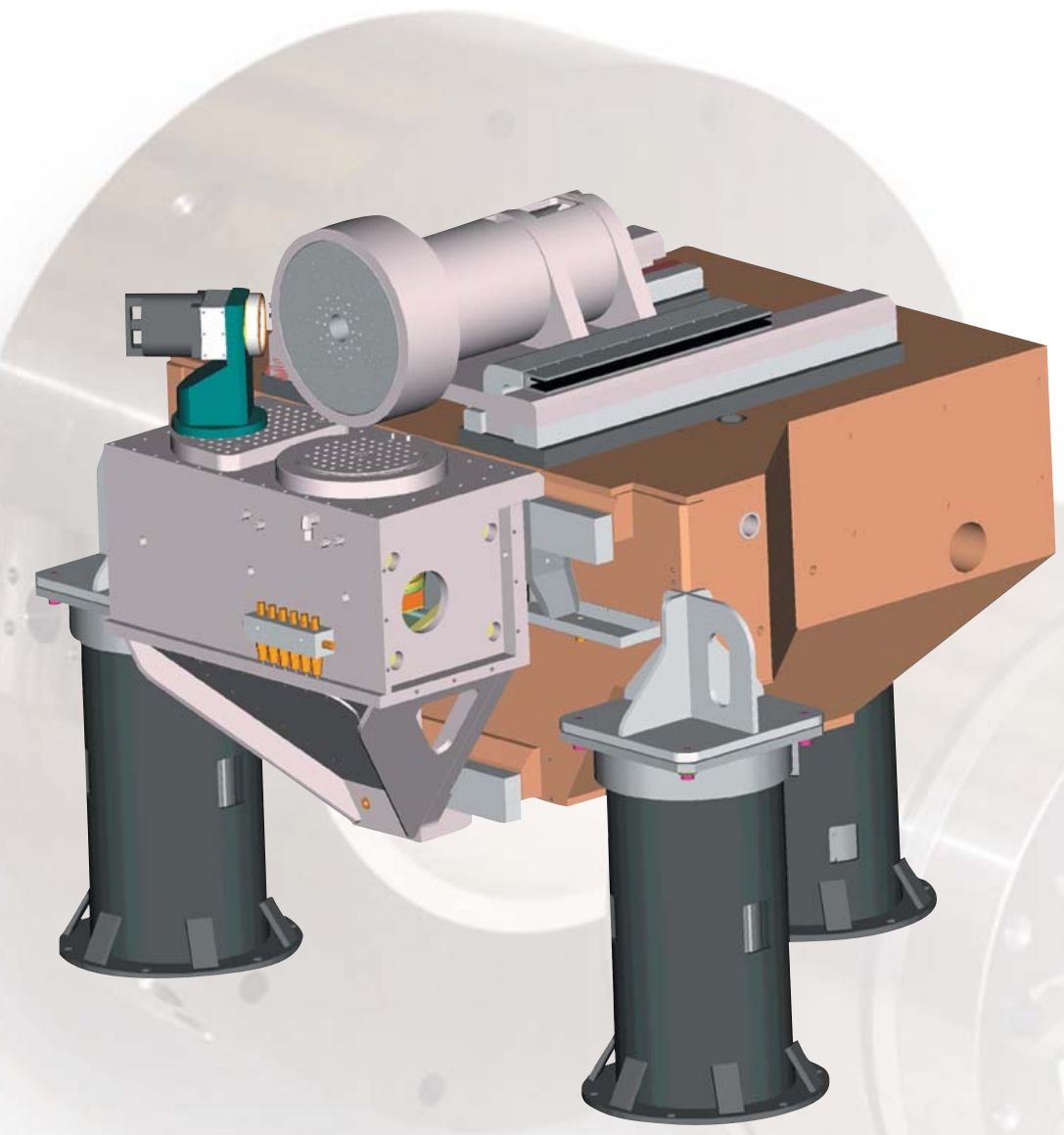

\section{FY2005 Proposed Work}

In FY2005, we will assemble and test the porous ceramic bearing spindle to confirm that it meets the original requirements shown in the table. A final report will be written and the results will be presented at the American Society for

Precision Engineering annual conference.

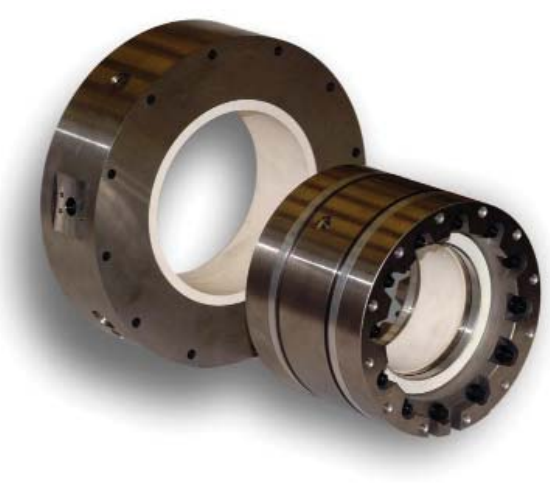

Figure 2. Front journal and rear journal and thrust bearing assemblies. The light color components are made of porous ceramic.

Figure 1. Conceptual drawing of the new Precision Optical Grinder and Lathe (POGAL) machine, the next-generation machine tool at LLNL. 


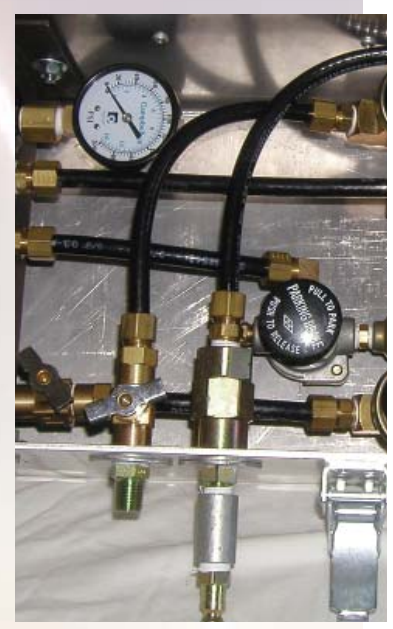



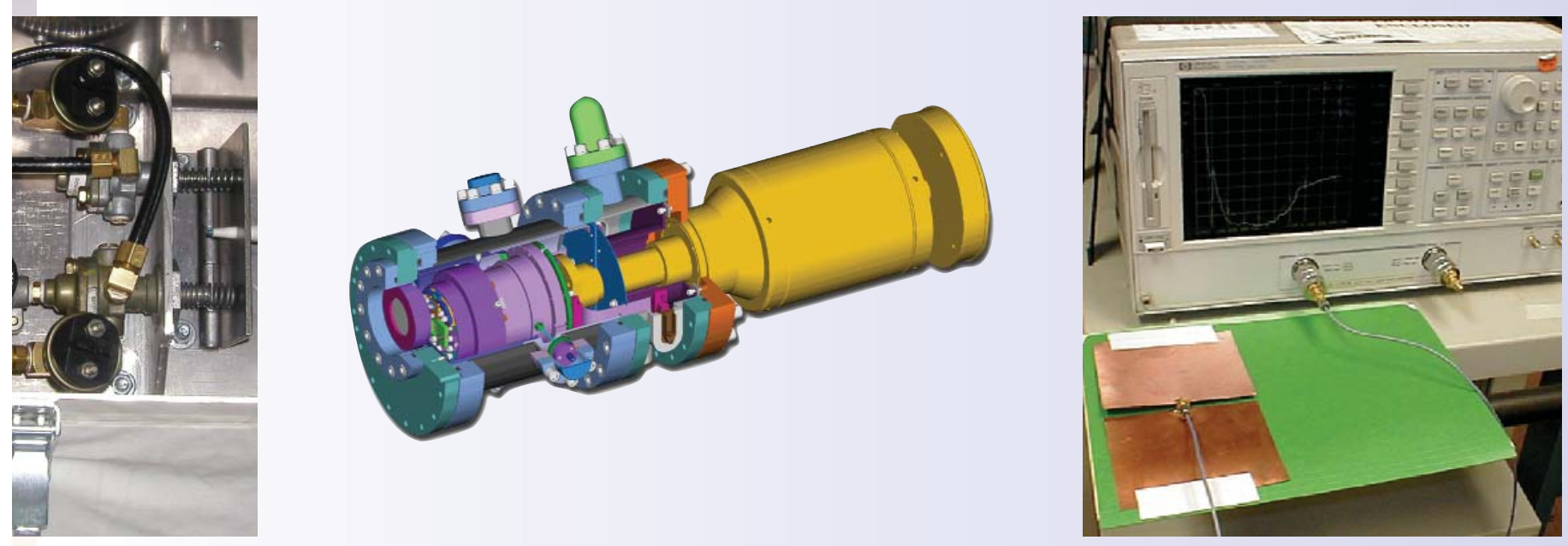

Center for

Complex Distributed Systems 


\section{Air Valve Truck-Stopping Device}

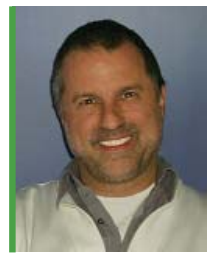

For more information contact Mark Strauch

(925) 422-1469, strauch1@|lnl.gov

he air valve truck-stopping device (AVTSD) has two main functional characteristics.

First, like the latest mechanical impact TSD units, the AVTSD requires two successive impacts before it is actuated and applies the service air brakes on a truck or trailer independent of any action by the driver of the vehicle.

Second, the AVTSD can be programmed such that a fixed period of time must transpire between the first and second impact before the AVTSD is activated to stop a vehicle. This adjustable time delay can be from zero to twenty seconds. Any second impact before the time delay has transpired will not activate the AVTSD.

Clearly, the AVTSD described above must have at least three operational states. These are 1) fully reset and ready for first impact; 2) impacted once and waiting for the time delay to pass so that a second impact will activate; and 3) impacted a second time after the time delay has passed. When the AVTSD is in the third state, it is activated and causes the service brakes on a vehicle to be applied. It is also desired that the AVTSD can be easily reset to the first state by those authorized to do so.

\section{Project Goals}

One of the milestones of this project was the demonstration and testing of an AVTSD.

\section{FY2004 Accomplishments and Results}

Our goal was achieved by a pneumatic system that behaves like a three-state electronic circuit with memory. The memory is contained in a small air tank that requires a specified time to charge up to a preset air pressure after the AVTSD unit has been impacted the first time. The air tank behaves like a capacitor in an electronic circuit. The logic elements in the system are accomplished using standard manually operated control air valves that have two stable states, ON and OFF.

Each of these control valves has an air input port, two air output ports, and an exhaust port. In the OFF state, no air pressure is passed from the input port to the output port(s). Any air pressure in the output port (line) is exhausted to ambient air pressure through the exhaust port that is co-linear with the activating plunger of the air valve. In the ON state, air pressure is passed from the input port to the output port and the exhaust port is disconnected from the output port.

The advantage of using these standard control valves is that these valves are mounted on millions of trucks and are, therefore, known to truck drivers.

The figure shows the AVTSD enclosure and its components. This AVTSD is constructed using only three Bendix air control valves, two model PP-1 valves and one model PP-8, plus a small air tank, and standard air hoses to connect the valves and the air tank. This assembly is contained in a locked aluminum enclosure, A, configured to be mounted under the chassis of any truck or trailer. Impact force on the rear bumper of a truck or trailer is transmitted to the AVTSD unit by a flexible control cable of any length.

Normally, the AVTSD enclosure is locked so that only authorized personnel can open the lid, B, and press the vertical PP-8 valve to reset the unit after it has been activated. 


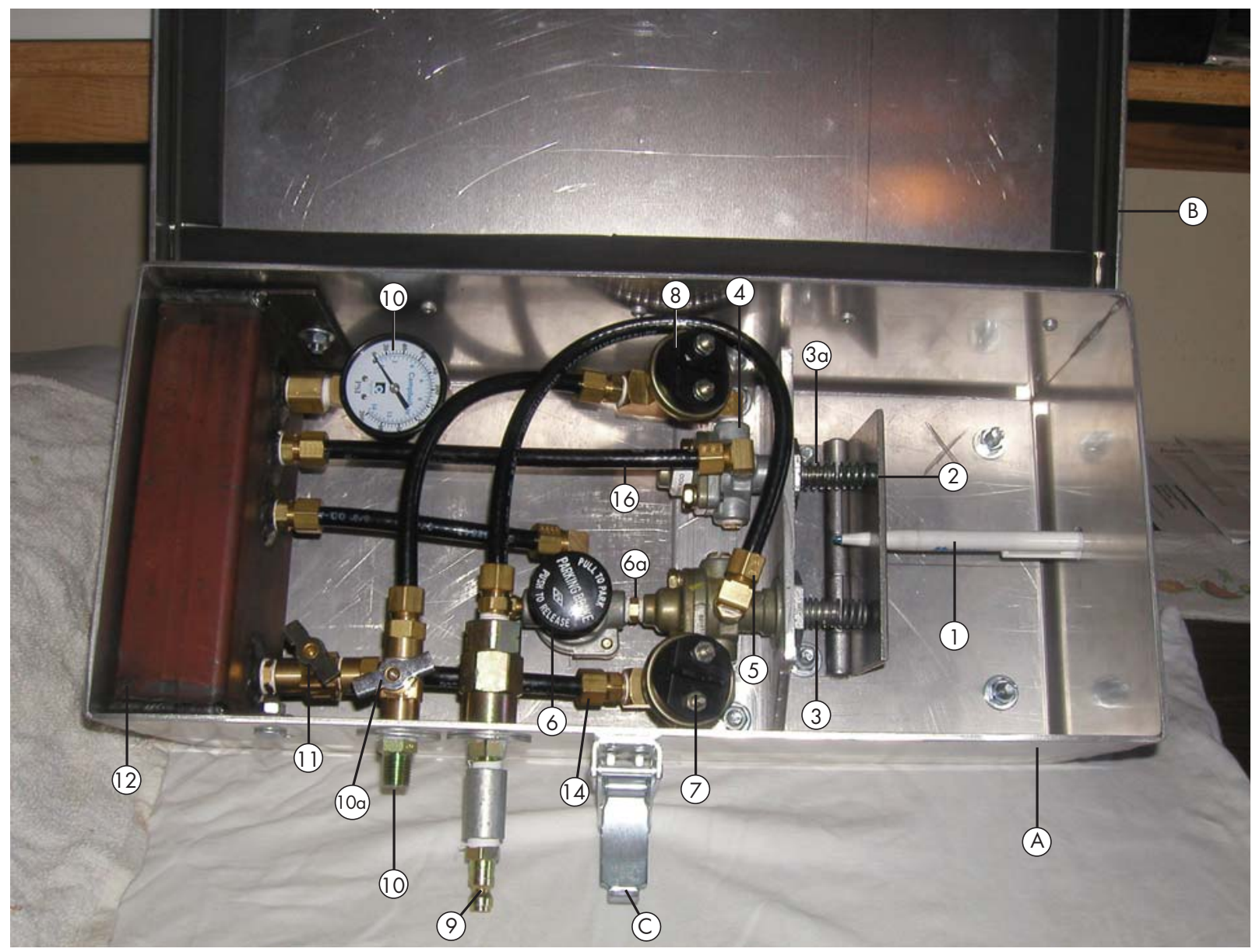

The AVTSD. The end of the mechanical control cable is the white shaft, 1, entering through a hole in the right end of the enclosure. This shaft pushes on the hinged plate, 2, which in turn pushes the plungers, 3 and $3 a$, of two air valves, 4 and 5 , mounted in the horizontal position. Return springs are mounted on the air valve plungers so that the hinge plate returns to the OFF position whenever the impact force transmitted by the control cable is removed.

The operating cycle is as follows: a first impact on the hinged plate, 2, causes the PP-8 valve, 5, to be set in the ON position. Main supply air pressure, 9, then flows through valve 5 to build up air pressure in the air tank, 12. The setting of the adjustable valve, 11 , determines the delay time before air pressure reaches the preset valve. Until air pressure in the tank exceeds the preset minimum level for the PP-1 valve, 4, the PP-1 valve will not stay in the ON position, no matter how many times it is depressed by additional impacts on the hinge plate. When the air pressure in the tank exceeds the preset minimum value, the next impact on the hinge plate will cause valve 4 to stay in the $0 \mathrm{~N}$ position and send air pressure to the air brake activation line, 10, thus activating the AVTSD. 


\section{Analysis of Emerging Mote
Technology as a Distributed \\ Analysis of Emerging Mote
Technology as a Distributed Sensor Platform}

$T^{\mathrm{T}}$ his project was a single year effort to investigate the potential benefits and applications of an emerging technology referred to as motes (or more accurately macro motes (Figs. 1-3), given current achievable sizes with commercial offthe-shelf components). Motes are small, inexpensive, low-power devices that can automatically form ad hoc wireless communication networks among themselves when deployed in an indoor or outdoor environment. When sensors are connected to their I/O ports, motes become a quick and cost effective platform for distributed sensing applications.

The motes themselves have embedded networking and routing code, which allows them to support various network topologies, from simple star patterns to more complex multi-hop mesh and partial mesh networks. Typically powered by one or two small batteries, motes operate with lowduty cycles, and sleep the majority of the time to conserve battery life. They awake only when a sensor reading is to be transmitted or when a command or message from another mote is to be received.

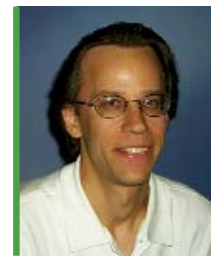

For more information contact Kenneth M. Masica (925) 422-4500, masical@|ln.gov

The basic components of motes include a battery power source, a micro-controller, an RF transceiver, one or more analog-todigital channels, and one or more digital I/O channels. Communication, applications, and management software can be preprogrammed into the mote or written by the user. Motes typically transmit at the 1-mW power level and achieve $20 \mathrm{~m}$ to $100 \mathrm{~m}$ distance, depending on antenna configuration and the RF propagation characteristics of the environment in which they are deployed.

Mote technology began as a DARPAsponsored "smart dust" project that envisioned miniature MEMS-based devices on the order of $1 \mathrm{~mm}^{3}$ in size with an integrated solar cell, thick film battery, analog sensor(s), a small processor, and optical transceiver. Work toward that goal continues, with $100-\mathrm{mm}^{3}$ prototypes created and tested. Currently, however, a trend to produce larger macro mote devices using inexpensive commercial components has emerged. Companies are producing third generation products and others have created mote evaluation kits for customers to
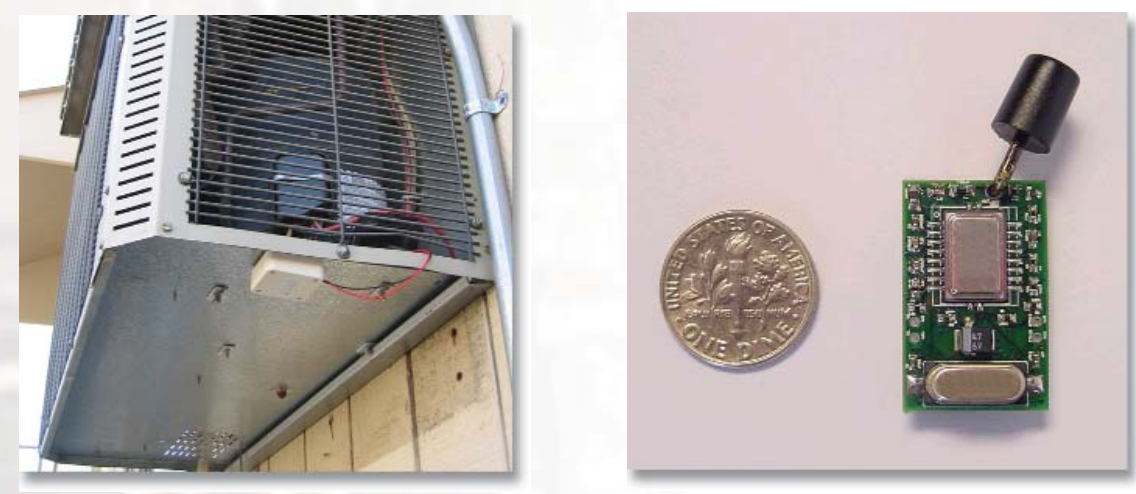

Figure 1. (Left) iBean wireless mote monitoring heat pump current draw; (right) iBean unpackaged mote next to dime for size comparison. 
conduct inexpensive testing and prototyping. There is also an open source software environment available for motes (Tiny OS), that includes an operating system, distributed database system, routing algorithms, and various programs for distributed sensing applications. Users can modify the code as necessary or create their own custom applications using the nesC programming language, an open source language developed for the mote technology.

\section{Project Goals}

The project goals were to characterize the current capabilities of macro mote technology offerings in terms of performance, functionality, sensor integration, power management, and system management; perform a cyber security analysis of mote networks and create security policy guidelines for wireless mote technology; gain first-hand knowledge and experience with macro motes through indoor and outdoor pilot implementations in the areas of environmental monitoring and building automation applications; and work to introduce mote technology and explore potential uses of motes to meet LLNL programmatic objectives.

\section{Relevance to LLNL Mission}

Motes may be good candidates to replace or augment current manual or expensive monitoring applications at LLNL, such as groundwater or surface water monitoring, environmental testing for contaminants, and energy management. Other potential applications include security, device tracking, and chemical/biologi$\mathrm{cal} /$ radiation sensing systems.

\section{FY2004 Accomplishments and Results}

In this fiscal year, we have conducted security vulnerability analysis of mote networks and created guidelines regarding their use and deployment at the Laboratory; characterized the performance, reliability, and sensor integration features of several mote technology offerings; and performed system integration of mote hardware, sensors, and software to implement several prototype mote networks. The networks include: a 12-node outdoor mote pilot network to interconnect ground well monitoring locations; a 17-node indoor/outdoor mote network to monitor building environmental conditions and HVAC equipment parameters; and a 10-node indoor standards-based mote network compliant with the IEEE specification for this technology.

\section{Related References}

1. "Smart Dust: Mighty Motes for Medicine,

Manufacturing, the Military and More,"

http://www.nwfusion.com/nltechexec350, 2003.

2. "Smart Dust Project: Autonomous Sensing and

Communication in a Cubic Millimeter," http://robot-

ics.eecs.berkeley.edu/ pister/SmartDust/

3. Collection of Links to Wireless Sensor Network Research and Design Topics:

http://www.research.rutgers.edu/ mini/sensornet works .html

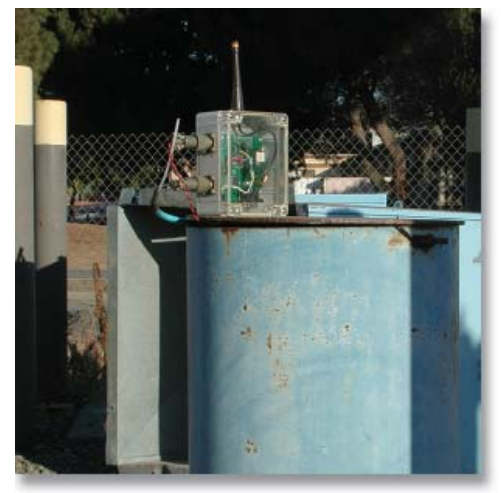

Figure 3. Repackaged dust sensor with integrated pressure transducer on top of ground well.

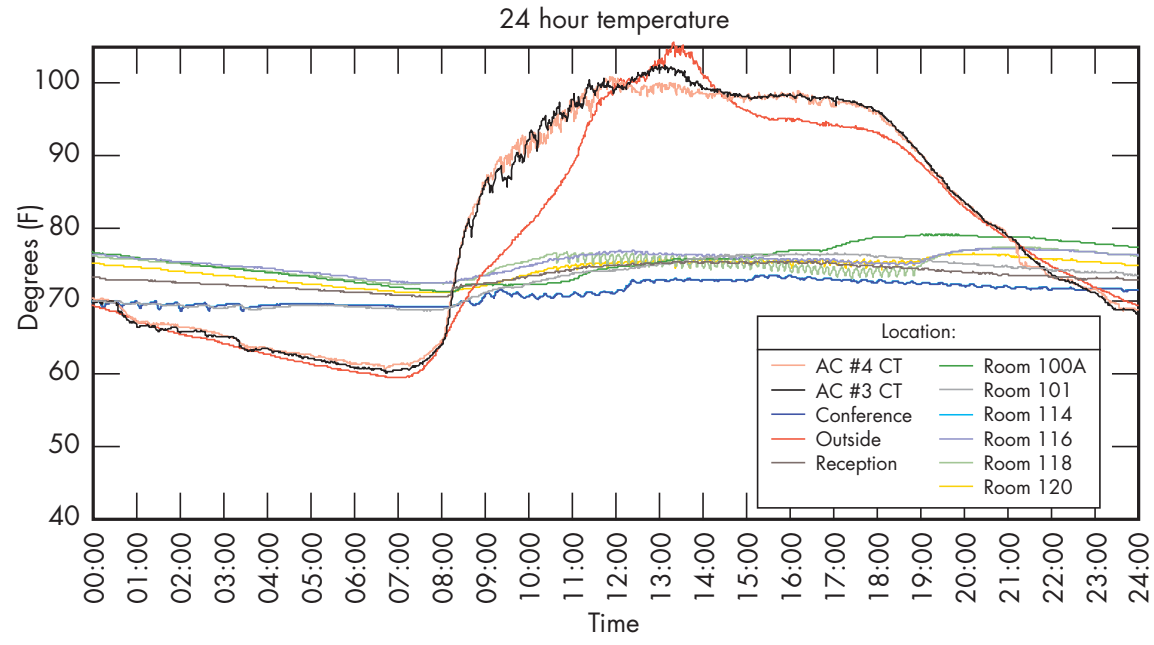

Figure 2. Data from software written to collect and graphically display temperature readings for the indoor/outdoor building monitoring mote network. 


\section{Cooperative Discrimination Sensor: Detecting and Tracking Human Activity}

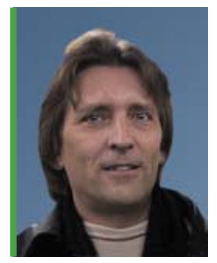

For more information contact Richard R. Leach, Jr. (925) 423-3351, leach1@|ln..gov

The first year of this two-year effort focused on combining or fusing existing UWB radar circuits, evaluating interference issues, testing radar performance, and applying signal-processing algorithms to detect, track, and discriminate basic human activity, such as respiration rate. Current sensors were evaluated in a precision test-bed to characterize their performance and determine how they perform in concert for intrusion sensing and characterization in network applications.

\section{Project Goals}

Our goal in the second year was to focus on combining the fused sensors into a three-node test-bed network. This testbed network will be used to evaluate system performance, demonstrate cooperative

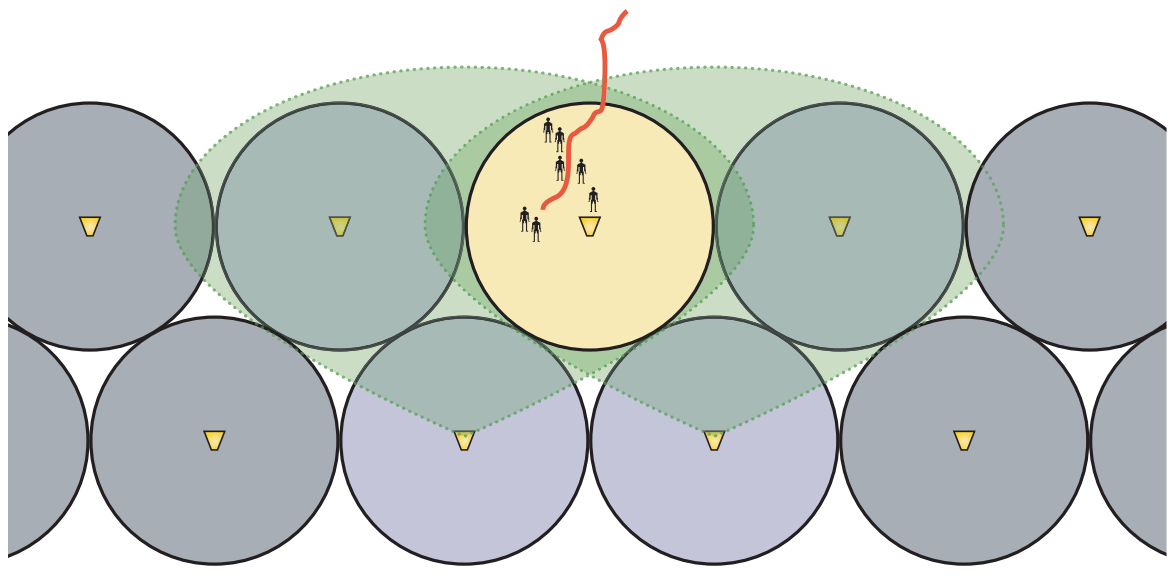
discrimination in a network setting, and apply advanced signal-processing techniques to test various tracking, detection, and discrimination algorithms.

\section{Relevance to LLNL Mission}

From this work the Laboratory will gain a multi-function, integrated radar sensor that is capable of detection, tracking, and discrimination in a single package. The package can be used in a deployable perimeter security network that operates at power levels previously unobtainable by conventional methods. This project has strong potential for antiterrorism, border, and battlefield monitoring, and

Figure 1. CDS radar network system. The detection area is shaded in yellow; the tracking area is shaded in green; and the intrusion path for tracking and identification of human activity is indicated in red. Nodes with no activity are shaded in gray and are in an extremely low-power state (quiescent). 


\section{FY2004 Accomplishments and Results}

UWB radar units were evaluated in a precision test-bed to characterize their performance. Radar data was collected over timed intervals and processed to measure interference, efficacy of detection performance, and extraction of respiration signals.

In addition, tracking, or swept-range radar units were used to calculate the position of a target as it was mechanically moved along a known path (see Fig. 2). Position estimation errors were calculated and the overall tracking performance of the radar was determined for each type of target. These tests were repeated using targets with a radar cross-section (RCS) similar to a human target, including mechanical targets and human subjects.

Human discrimination using RCS from radar circuits has been studied with limited results; however, this work led to the idea of using motion artifacts from human activity, such as velocity, breathing, and heartbeat, for discrimination and monitoring.

A brass-board system was assembled and tested with the best performing radar
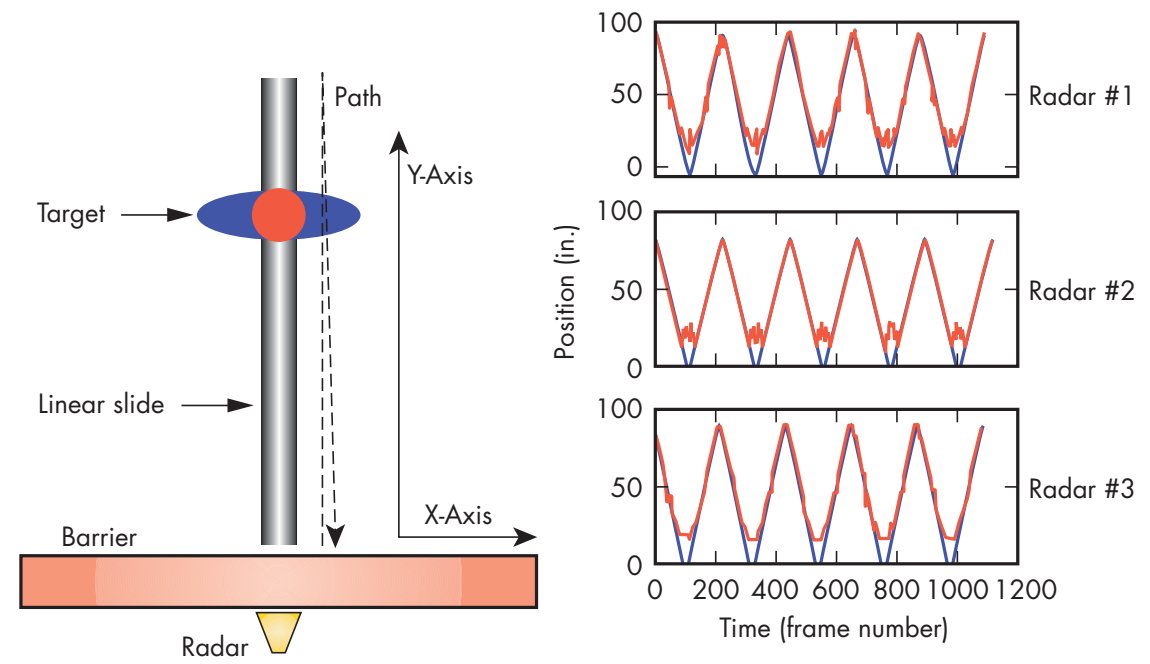

Figure 2. Results from tracking comparison tests, performed using a linear slide and a calibrated target (metal plate) to evaluate the performance of each radar circuit type. Plots indicate known position (blue) and radar-determined position (red).
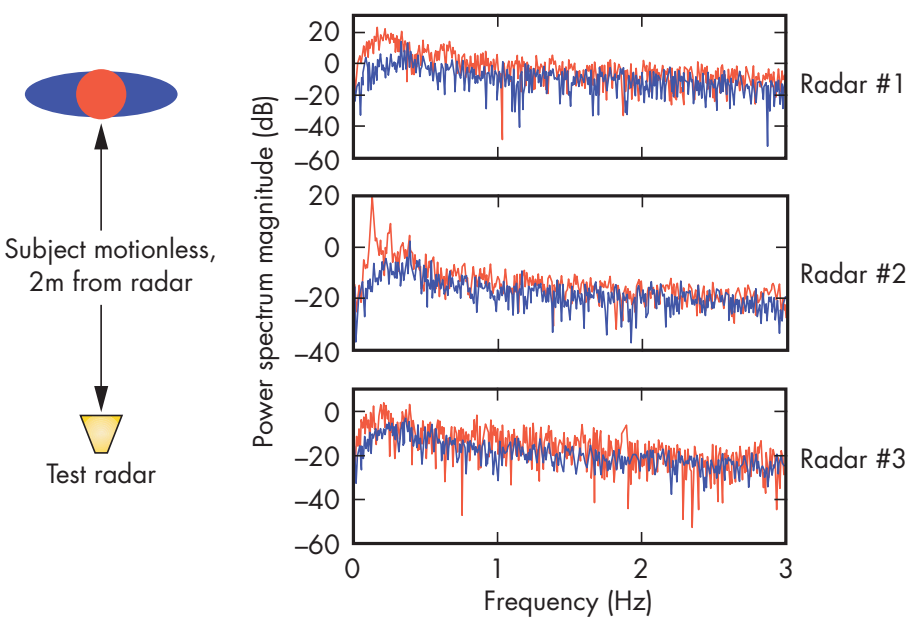

Figure 3. Results of respiration rate comparison tests to evaluate the performance of radar circuit types. Spectral plots indicate background noise (blue) and radar-determined respiration rate (red). 


\section{Long-Range Ultra-Wideband Radio-Frequency Identification}

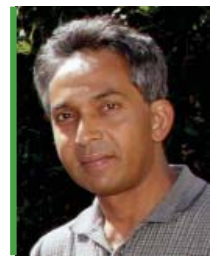

For more information contact Farid U. Dowla (925) 423-7579, dowla1@|lnl.gov

UWB RFID technology would encompass UWB pulse generation, remote powering of tags at longer distances, the steering of antennae, and defining an infrastructure matching the particulars of the environment.

Even state-of-the-art RFID systems, such as automatic identification and data capture (AIDC), are essentially short-range (less than $2 \mathrm{~m}$ ), using narrowband inductive systems. On the other hand, target objects in areas of interest are often located in cluttered or harsh environments, so they are best tackled by wideband RF pulses.

The class of problems we plan to focus on is the use of labels in which passive RFID devices are imbedded onto disk drives, removable media, file folders, and other materials that physically contain classified or otherwise sensitive information. Ultimately, much will depend on whether we can achieve a cheap package with useful ranges and spatial resolutions. There are some concerns with the introduction of RF signals in classified environments, but the use of UWB signals may overcome many of these obstacles.

\section{Project Goals}

Generally, we plan to take advantage of UWB RF pulses to encompass: 1) longer range of tag interrogation, given equal average power from the interrogator (or conversely, greater range in sensitivity); 2) more immunity to signal degradation and multipath effects; 3 ) a higher degree of security and immunity to eavesdropping; 4) a greater potential for anti-collision in multi-tag environments; 5) more uniform coverage of a volume of space; and 6) the ability to focus the tag interrogation to a localized point in space. 
Another important goal is to demonstrate the utility of integration of the appropriate COTS technology with LLNL-proprietary covert long-range UWB tags for CREM monitoring. In a phased, low-risk approach, we propose to implement passive UWB tags and tag readers with a range of about $10 \mathrm{~m}$. COTS systems will also be custom deployed for laboratory testbed evaluation and performance comparison.

\section{Relevance to LLNL Mission}

On-demand real-time identification and tracking of devices using RFID tags is a powerful technology for improving Laboratory security. The need to make some classified items fully accountable has been identified. The vulnerabilities of commercial RFID products are only beginning to emerge.

\section{FY2004 Accomplishments and Results}

We have solved many of the key problems, described briefly as follows: system and block configurations of the UWB RFID have been completed, with simulation and analysis (see Figs. 1 and 2 ); the precise generation of a highpower UWB sub-ns duration transmitters have been demonstrated (see Fig. 3); the remote powering circuit that will switch on the tags (or controllers) for response has been completed; RFID systems for tests and analysis have been surveyed and acquired; theoretical analysis and laboratory experiments for range vs. peak voltage received at the tags for remote powering have been completed.

Initial experiments indicate that both the tag readers and the tags themselves will be small and eventually low-cost systems. The passive or semi-passive tags can be planted in places of interest without intervention for many years. Most of the unresolved technical problems that remain are concerned with the logistics of the deployment and application issues, such as speed of reading, number of tags, continuous or event-driven monitoring, and other integration, interface, testing and performance issues.

\section{Related References}

1. Finkenzeller, K., RFID Handbook, John Wiley and Sons, New York, New York, 2003.

2. Dowla, F. (Ed.), "The Basics of Radio Frequency Identification Technology," Chapter 14 in Handbook of RF and Wireless Technologies, Elsevier, 2004. 3. Siden, J., P. Jonsson, T. Olsson, and G. Wang, "Performance Degradation of RFID System Due to Distortion in RFID Tag Antenna," Microwave and Telecommunication Technology, pp. 371-373, 2001. 4. Bansal, R., "Coming Soon to a Wal-Mart Near You," IEEE Trans. on Antennas and Propagation Magazine, December 2003.

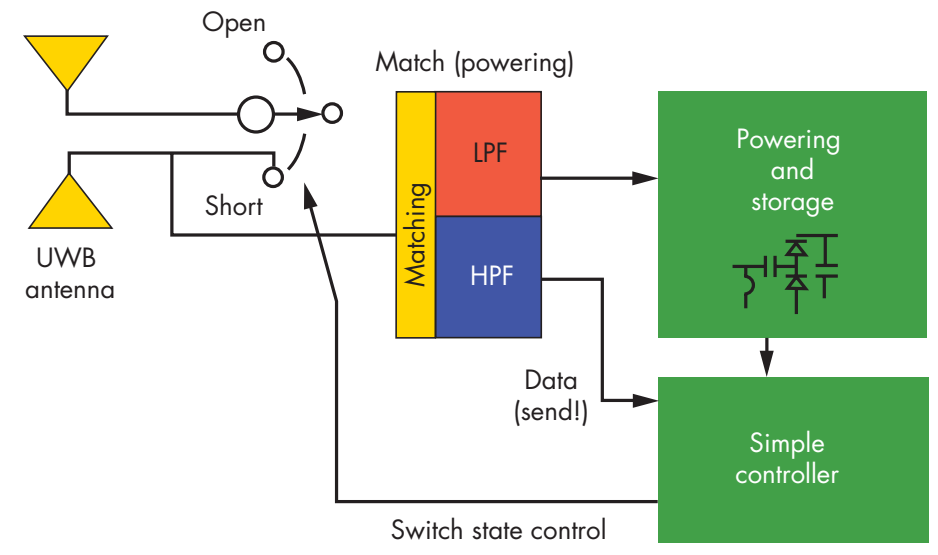

Figure 2. Block diagram of a long-range UWB tag.

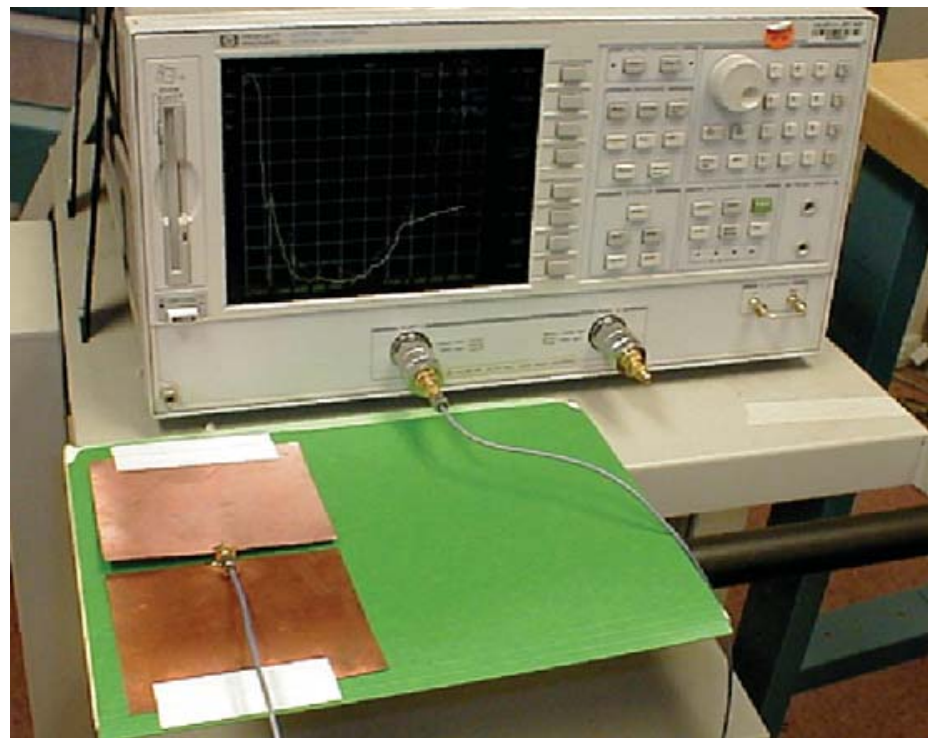

Figure 3. UWB tag antenna for folder identification. 


\section{Miniature Echelle Grating Spectrometer Cartridge}

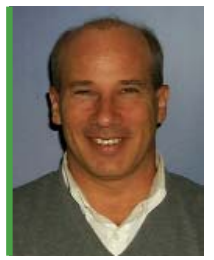

For more information contact Joel Bowers (925) 423-6877, bowers2@\|lnl.gov

high spectral resolution is attained in relatively high frame rates. Size reduction is achievable using a multipass architecture and immersion grating techniques.

\section{Project Goals}

This project performs initial scoping and exploration of a compact optomechanical configuration for an echelle spectrograph that has the high strategic potential of performing real-time, remotetarget forensics on warheads as they are being destroyed. We focus on the midwave infrared band for this study, with an approach that is extendable to other infrared bands, to collect potential nuclear warhead signatures of uranium, plutonium, tritium, and their potential compounds (see Fig. 1).

After down-selecting optical systems that meet these requirements, we sought to define precision mounts that maximize use of an existing cartridge-style mechanical infrastructure. This supports the vision of a relatively modest incremental investment of a new optical cartridge and controls that creates a completely new instrument. The final product is a feasible solution for an echelle spectrograph optical cartridge, including optical prescriptions, performance predictions, an FPA technology assessment, and an opto-mechanical cartridge packaging concept.

Figure 1. Locations of infrared emission spectra for nuclear warhead material signatures. 


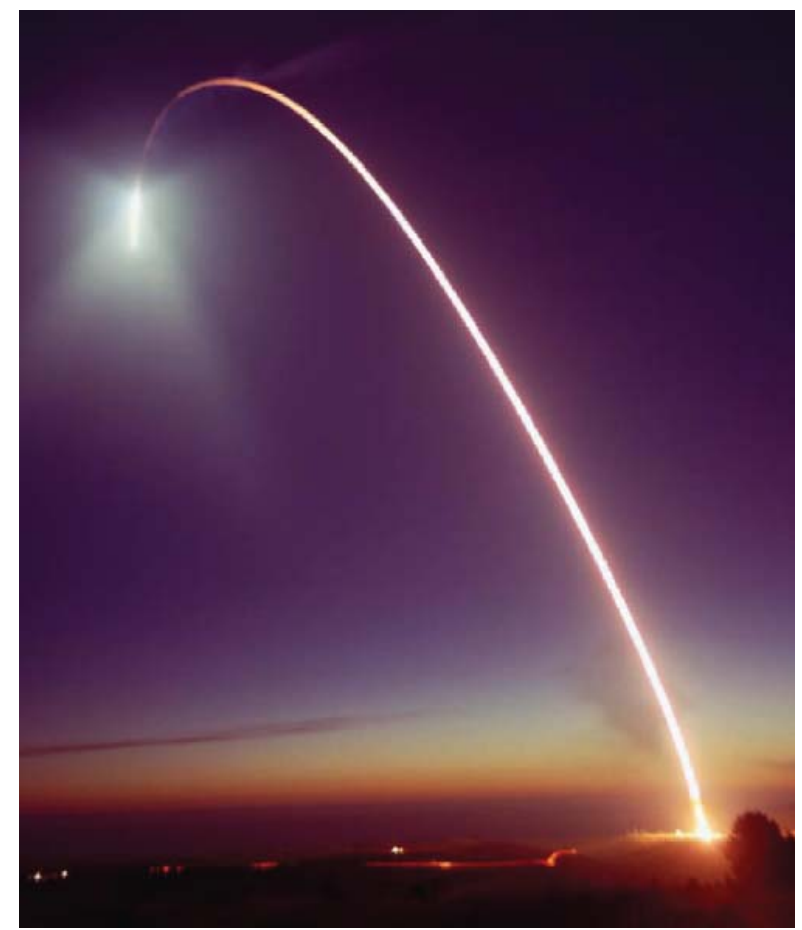

Figure 2. Nighttime missile launch at Vandenberg Air Force Base.

\section{Relevance to LLNL Mission}

LLNL's national security mission requires special multidisciplinary capabilities that are also used to pursue programs in advanced defense technologies.

Potential users include the U. S. Missile Defense Agency for warhead typing (see Fig. 2), Strategic Command, and the Special Operations Command for interdiction consequence assessments. The small form factor also makes this sensor attractive for other applications requiring high spectral resolution and low volume, weight, and power.

\section{FY2004 Accomplishments and Results}

In accordance with our project plan, the optical arrangement options that were considered were intentionally biased toward recent advances in miniaturizing hyperspectral instrument packaging infrastructure (dewar, vacuum system, cryogenic system).

A feasible optical solution was verified to have the required optical performance in the highly compact form factor (see Fig. 3). Optical volume was reduced by a factor of 60 . With a spatial image format of $3 \times 3$, an F-number of 4 , and a 256-×-256-pixel detector array of $30-\mu \mathrm{m}$ pixels, we verified that the diffraction-limited model meets the waveband requirement of 3.3 to $4.5 \mu \mathrm{m}$, and spectral resolution of $0.27 \mathrm{~cm}^{-1}$. Infrared emission spectra for nuclear warhead materials were identified. For the midwave version studied, spectral signatures of high explosive detonation and tritiated water fall within the sensor passband.

\section{FY2005 Proposed Work}

Further optimization of the wavelength range will tailor the baseline system toward signatures of interest. Diffraction efficiency checks will determine transmittance vs. wavelength. Reformatting the 3-x-3 array to a 9-x-1 array for the sensor entrance slit will require additional optical elements directly in front of the entrance slit. A preliminary optical tolerance study will be performed.

Commercial off-the-shelf focal plane array technologies will be investigated for feasibility at this form factor. A mechanical study will determine the extent that the existing hyperspectral optical cartridge can be used to build a new cross-dispersive system, and immersion gratings will be checked for manufacturability. New concepts will be generated based on this gap analysis, to determine the complete scope of the effort needed for a prototype system. 


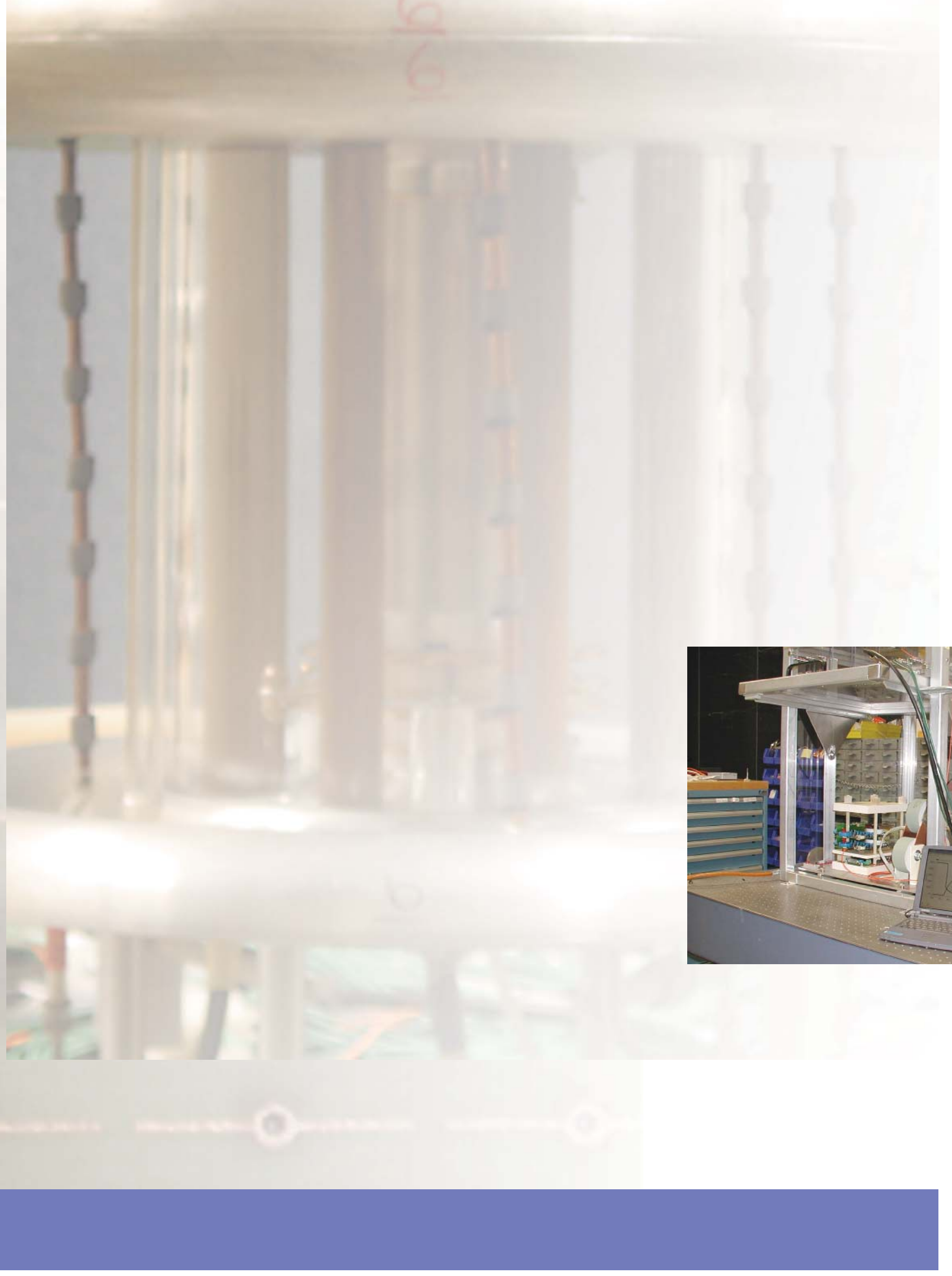



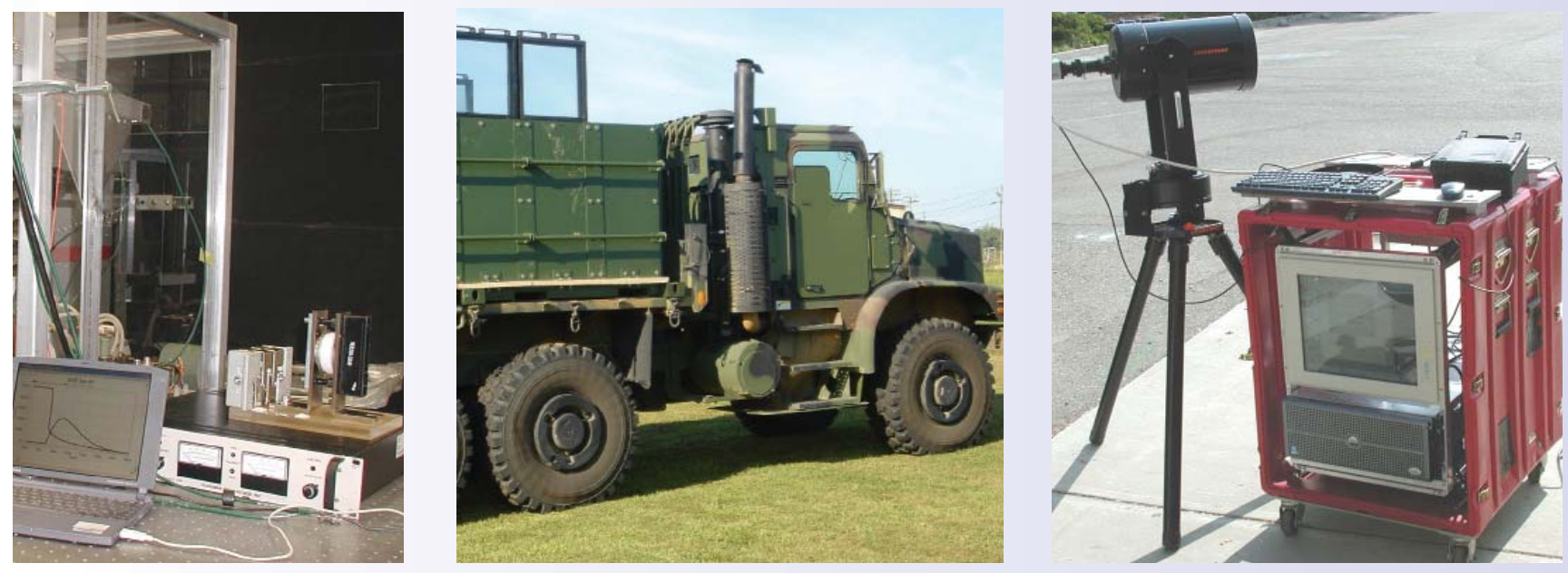

Other Technologies 


\section{Beryllium Surface Cleaning Process}

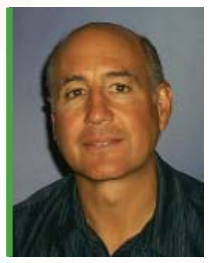

For more information contact Thomas M. Vercelli

(925) 423-4759, vercelli2@|ln..gov

LNL health and safety procedures require that Be parts, and machine parts such as tooling, which have come in contact with $\mathrm{Be}$, be cleaned to a level of $0.2 \mu \mathrm{m}$ $\mathrm{gm} / 100 \mathrm{~cm}^{2}$ or less for free release to non-Be work areas. Currently no agreedupon procedures exist for this cleaning. A standardized cleaning process for Be parts with a documented cleaning efficiency can establish a Be part as an article, with out sampling each part.

An ultrasonic cleaning process (Fig. 1) using a water-based detergent appeared to be a good candidate to evaluate for use at LLNL. A documented and agreed-upon cleaning process used by other DOE Contractors would minimize concern when accepting their Be parts.
An appropriate statistical analysis would permit periodic or spot-checking of cleanliness levels, rather than evaluating every piece.

\section{Project Goals}

The goals of this project are: first, to evaluate the potential of an ultrasonic cleaning process as a DOE standard for cleaning of Be parts, with an efficiency that establishes a Be part as an article; and second, to investigate the availability and cost of a commercial ultrasonic cleaning unit to be integrated into LLNL's Be facility.

\section{Relevance to LLNL Mission}

This project supports Integrated Safety Management principles. If successful,

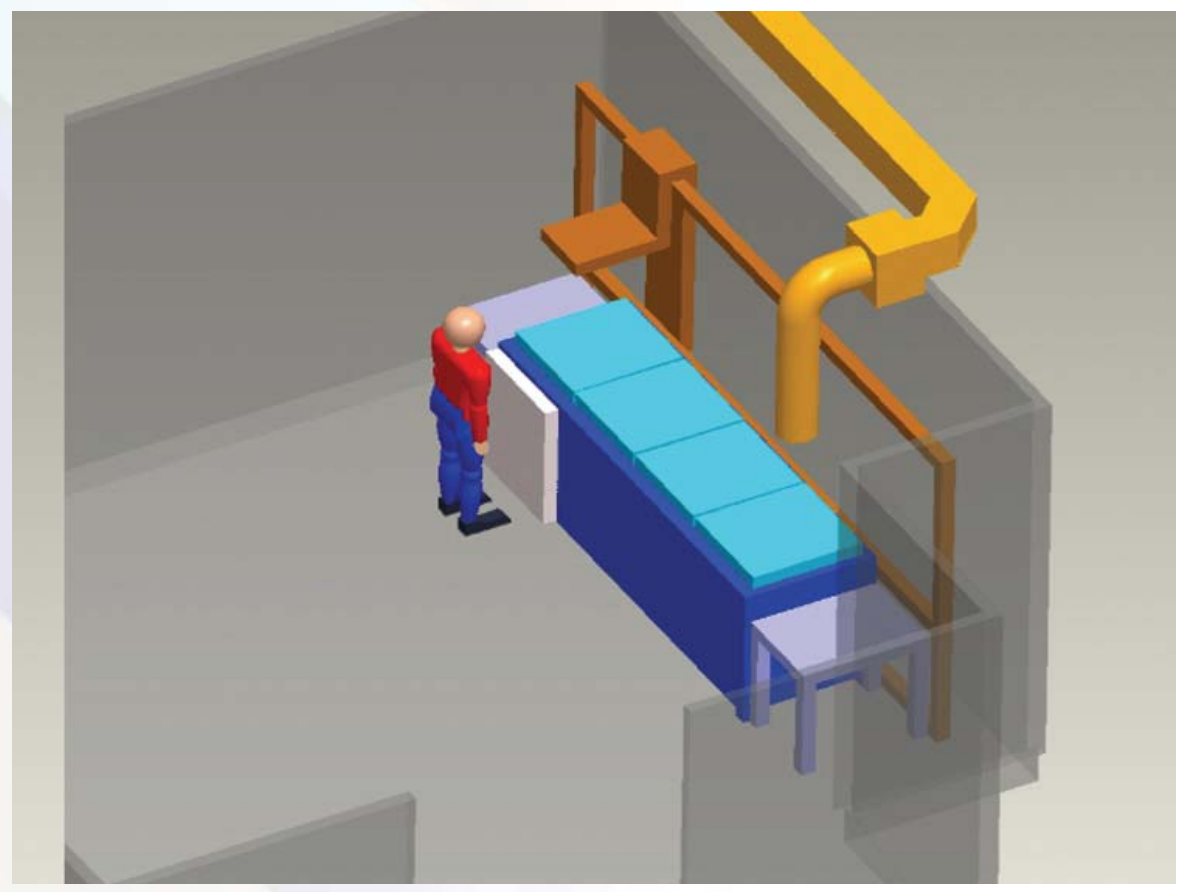

Figure 1. Concept of an ultrasonic cleaning system in LLNL's Be Facility. 

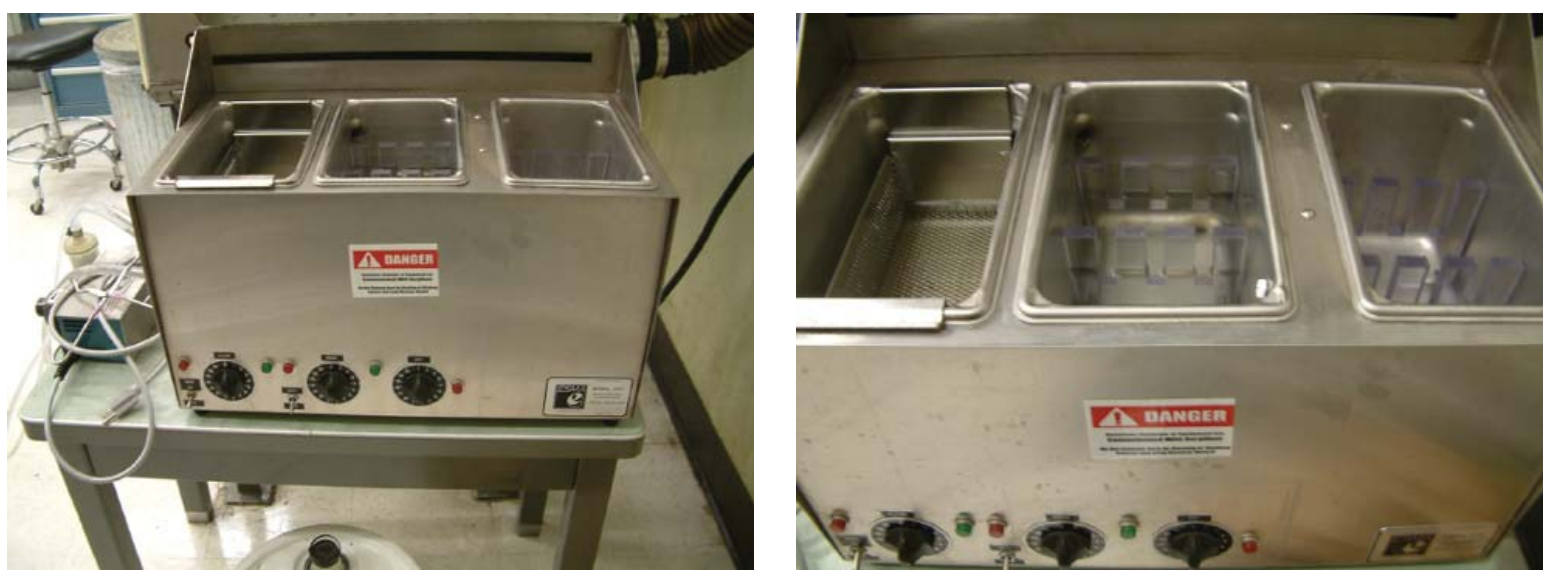

Figure 2. Two views of the Colster-3 ultrasonic cleaning tank.

worker safety will be improved, and Be parts will be handled in a more safe and expedient manner. Some LLNL beneficiaries of this project are Hazards Control, DNT, and NIF

\section{FY2004 Accomplishments and Results}

During this project a Colster-3 benchtop ultrasonic cleaning unit (Fig. 2) was modified to provide local ventilation and re-circulation/filtration of rinse water. The ventilation was integrated into the HEPA filtration system.

Jem Chemical Co., \#68XLF water-based cleaning detergent was purchased for the initial evaluation. Nine Be test pieces, approximately 6 in. $\times 2$ in. $\times .25$ in., were cut and machined. A swipe was taken from half of the area of each side of the test sample before and after cleaning. Each test part was individually put into the cleaning bath for $15 \mathrm{~min}$. The cleaning agent was maintained at $130{ }^{\circ} \mathrm{F}$. After cleaning, the part was placed into the rinse tank for $15 \mathrm{~min}$, and then into the dryer tank for $15 \mathrm{~min}$. The process was repeated for all nine test parts. Swipes taken after the cleaning cycle revealed that, while the Be parts showed improvement, they still contained removable Be particles above freerelease limits of $0.2 \mu \mathrm{m} \mathrm{gm} / 100 \mathrm{~cm}^{2}$.

The test parts were re-swiped and put through a second cleaning cycle, with little to no improvement in removal of Be particles.

A cleaning experiment using "shaped" test parts was also conducted. Be parts were machined with features such as slots, through-holes, and tapped holes. The shaped parts were put through the same cleaning process as the first nine test parts. Swipe results found Be particles above free-release limits. The highest values recorded were in the threaded holes.

An additional series of tests planned to vary parameters that may improve the cleaning efficiency is needed before any decision on the scale-up of this process can be made for application in the Be facility.

A study of outside (industrial) Be cleaning processes revealed that the requirements for Be contamination control in industry are not well defined and are typically ignored. Those that perform cleaning of Be manufactured items use standard degreasing techniques, with a small number using ultrasonic cleaning methods. The Be Control Program at LANL calls for largescale ultrasonic cleaning systems in their Be Facility but they do not attempt to reach the free-release threshold.

Large-scale commercial ultrasonic cleaning systems typically have tank sizes of 30 in. $\times 30$ in. $\times 30$ in. Systems differ by number and location of the frequency transducers, heater capacity, automation options, ventilation systems, and size. Both systems we evaluated used a $40-\mathrm{kHz}$ ultrasonic frequency and a filter system for the cleaning agent. One unit offered other frequencies, depending on the cleaning application. Both systems would require integration into the Be facility. This involves a HEPA ventilation system, a retention tank for proper disposal of the cleaning and rinse water solutions, a deionized rinse water make-up system, and electrical power. 


\section{Camouflaged Object Recognition}

W e have refined and furthered our analysis of the brainwave data obtained from our collaborator at the Biomagnetic Imaging Laboratory at the University of California, San Francisco (UCSF). We achieved 100-fold improvement in time resolution using a novel combination of wavelets and neural networks to extract signals of the brainwaves obtained from magnetoencephalography (MEG). These improvements provided technical results that will enable us to pursue the next phase of camouflaged object recognition.

\section{Project Goals}

The goal of the project is to extend our work done in the signal processing area to demonstrate improved signal extraction from extremely noisy brainwave data. The results will enable us to continue this

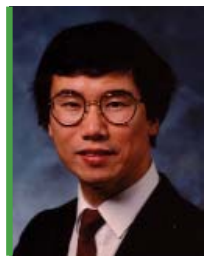

For more information contact Chi Yung Fu (925) 423-1175, ful@\|lnl.gov

novel technology of using brainwave monitoring to assist intelligence analysts and radiologists in finding "camouflaged," or subtle, objects and to improve human pattern recognition. Figure 1 shows how the traditional approach loses time resolution due to averaging of the MEG data from repeated stimulation. Our approach successfully extracted the brain signal epoch-by-epoch for each stimulation.

\section{Relevance to LLNL Mission}

This project is relevant to all Laboratory programs requiring pattern recognition. The most noticeable area is to assist analysts in the intelligence area. This is especially significant after September 11, 2001. Our results can have significant impact on homeland security, NAI, and the Department of Energy and Environment.

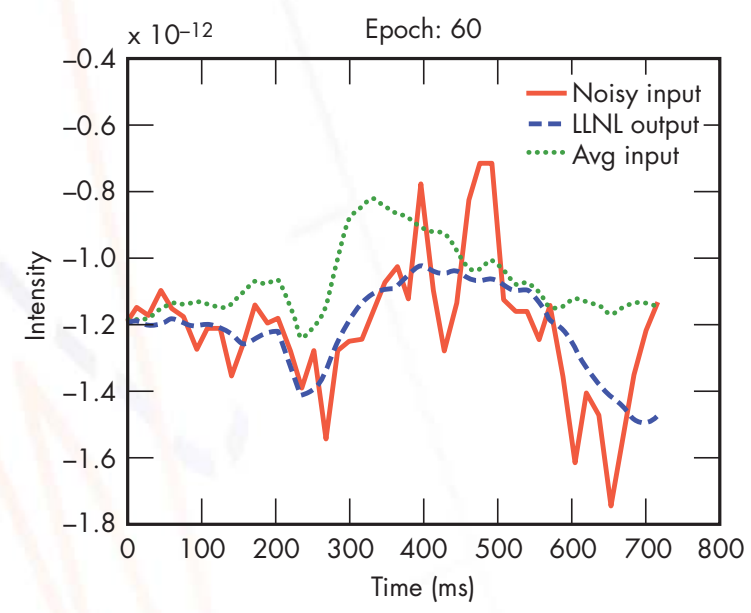

Figure 1. Comparison of results from various approaches to MEG data. 


\section{FY2004 Accomplishments and Results}

Up to 272 superconducting quantum interference devices (SQUIDs) were used to monitor the MEG brainwaves from the entire scalp. Different devices are activated differently, due to the differences in the responses from the various locations of the brain.

Each waveform from each SQUID consists broadly of two types of responses: one when stimulation is on, and one when stimulation is off. Both are embedded in a large amount of noise from different sources, making them almost indistinguishable. We assume no prior knowledge when the stimulation is presented. We also do not know what a clean waveform looks like. As a result, the neural network is an unsupervised one.

After wavelet preprocessing, the data were processed by a neural network that consists of two back-propagation networks, to learn the two different types of responses presented in our problem. Another network assigns different parts of the data space to these two networks. This divide-and-conquer technique improved upon our previous attempts. A 100-fold improvement in time resolution has been achieved. An invention disclosure is planned.

Figures 2 and 3 are some examples of our results. The decreasing response at $\sim 250 \mathrm{~ms}$, as a function of epochs from 1 to 120 , reveals that the brain was adapting to the repeated stimulation. Such adaptation could not be seen from either the raw or the conventional processed data in Fig. 3.

Analyzing satellite images and reading mammograms are essentially looking for a needle in the haystack, and thus, solving one will greatly facilitate solving the other, since our algorithm is not dependent on a particular application. We plan to continue our collaboration with UCSF's Biomagnetic Imaging Laboratory and with the University of California, Davis, Cancer Institute.

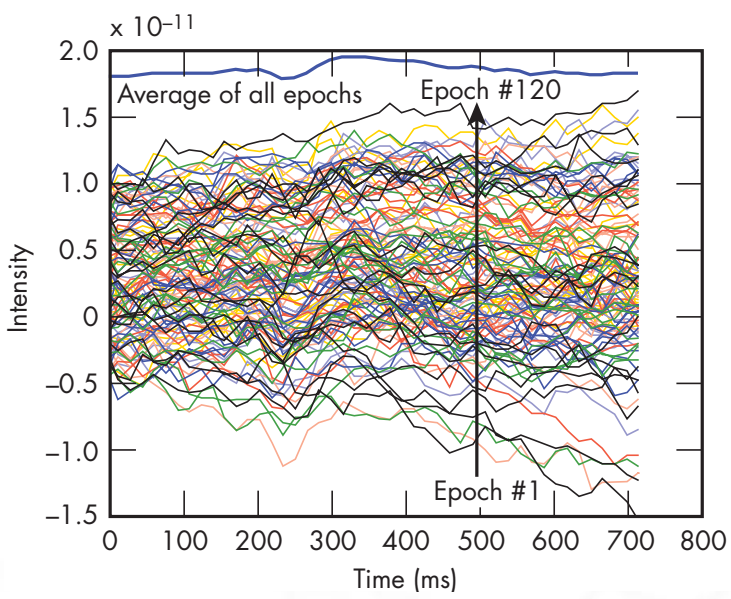

Figure 2. 120 epochs of raw MEG data corresponding to the processed data in Figure 3.

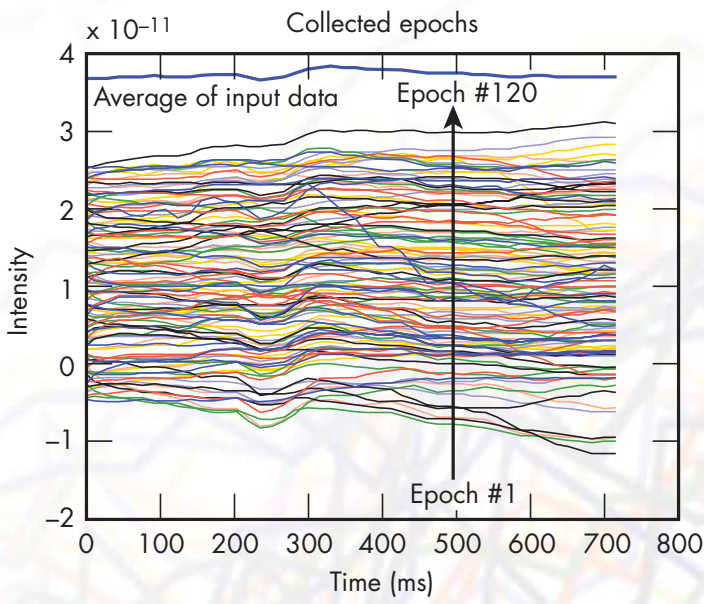

Figure 3. Brain adaptation to the audio stimulation at $\sim 250 \mathrm{~ms}$, apparent using LLNL approach. This allows us to explore the dynamics of the brain. 


\section{Demonstration of Armored Vehicle Technology}

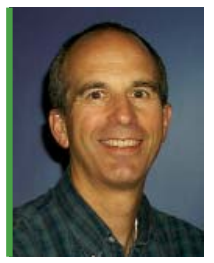

For more information contact Steven J. DeTeresa (925) 422-6466, deteresa1@|ln..gov

\section{Relevance to LLNL Mission}

This project is an example of the Laboratory's meeting a critical national mission during wartime. The work draws on the Laboratory's technical expertise in armor materials and mechanical engineering to rapidly construct and field an effective armor kit for soft-skinned military vehicles.

\section{FY2004 Accomplishments and Results}

A gun truck requires armor for the

\section{Project Goals}

The goal is to construct and field armor kits for construction of gun trucks using tactical vehicles having a cargo capacity of 5 tons or greater. The primary vehicles of interest are the FMTV, the M939 series, and the USMC MTVR trucks.

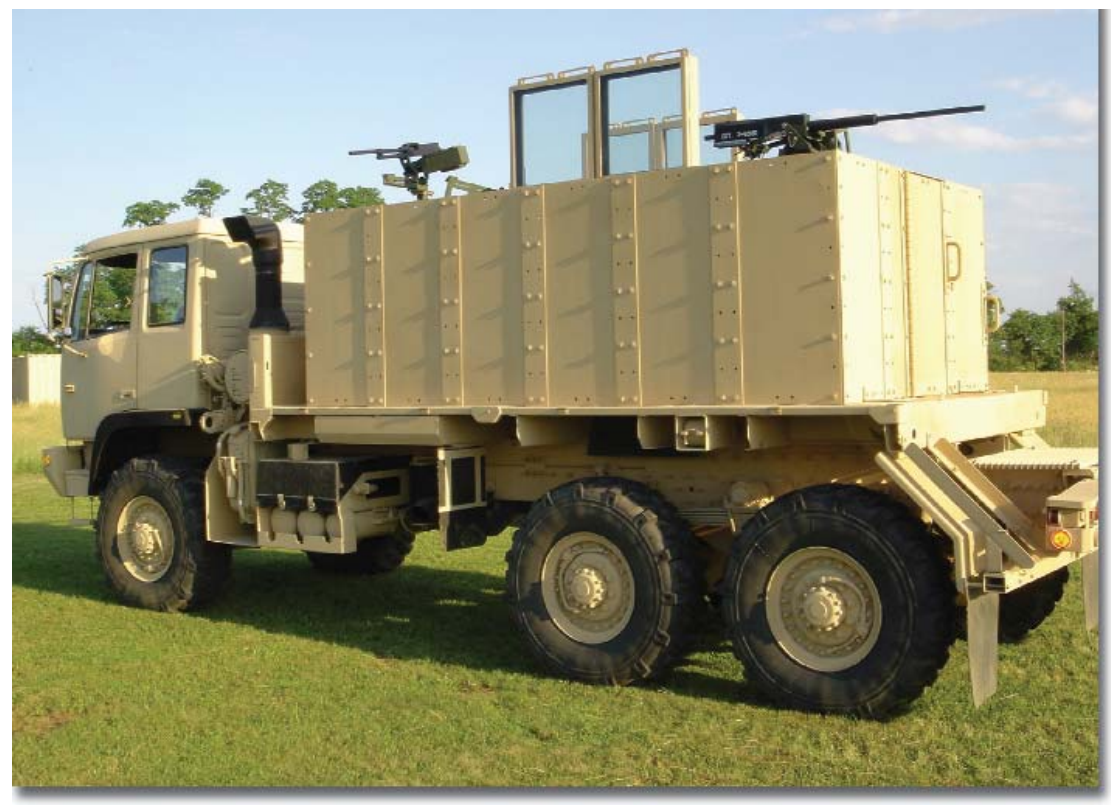

Figure 1. U. S. Army 5-ton FMTV gun truck as it was displayed at the Pentagon on June 4, 2004. truck cab and a "pillbox" for the bed. The armored box is typically equipped with three to four crew-served weapons, such as 0.50 caliber machine guns, to provide a high level of firepower to repel an ambush. Our first prototype gun truck box was tested for both ballistic and road performance at the U. S. Army Aberdeen Test Center (ATC). The armor consists of spaced armor steel and ballistic fiberglass panels. Sections of the armor were also tested at the ATC ballistics range and were shown to be effective in stopping all known indirect and direct fire threats.

In June, the gun truck (without the cab armor) was displayed at the Pentagon for high-ranking officials. This version of the gun truck was built on the U. S. Army FMTV truck, as shown in Fig. 1. Although the design borrows much if its attributes from the Vietnam versions of gun truck, we have additional features such as bolted construction, ballistic fiberglass shrapnel/spall shields, and ballistic windows.

We then built a gun truck from the older M939 trucks for the following reasons: 1) there are more M939 trucks than FMTV vehicles currently in the theater; 
2) the M939 truck is capable of handling 10 tons on-road, while the FMTV is rated for only 5 tons either on- or off-road; and 3) the cab of the M939 is much easier to armor. We worked with the USMC to modify a cab armor that they had already produced for the M939 series trucks, and built a cab armor kit in just one week. This first Livermore gun truck started running convoy escort missions on July 17. A photo of the truck leaving Camp Anaconda is shown in Fig. 2. Since that time, the gun truck has been escorting convoys at least three times a week. Feedback from the Army has been very positive, and 28 more LLNL gun truck kits have been requested.

We have also produced a second gun truck kit for the Marines' MTVR. The armored box is similar to the one developed for the U. S. Army, but the hardware to mount the box to the truck box and the cab armor are modified for this vehicle. The MTVR gun truck (Fig. 3) was assembled and demonstrated for the Marines at Camp Pendleton. It is currently undergoing road testing at ATC.

\section{FY2005 Proposed Work}

We will continue our efforts in vehicle armor technology with funding from DARPA. This follow-on effort will include producing 30 kits for gun trucks; further refinement for the USMC MTVR; and new, weight-efficient armor materials and structures for both vehicle and personnel protection.

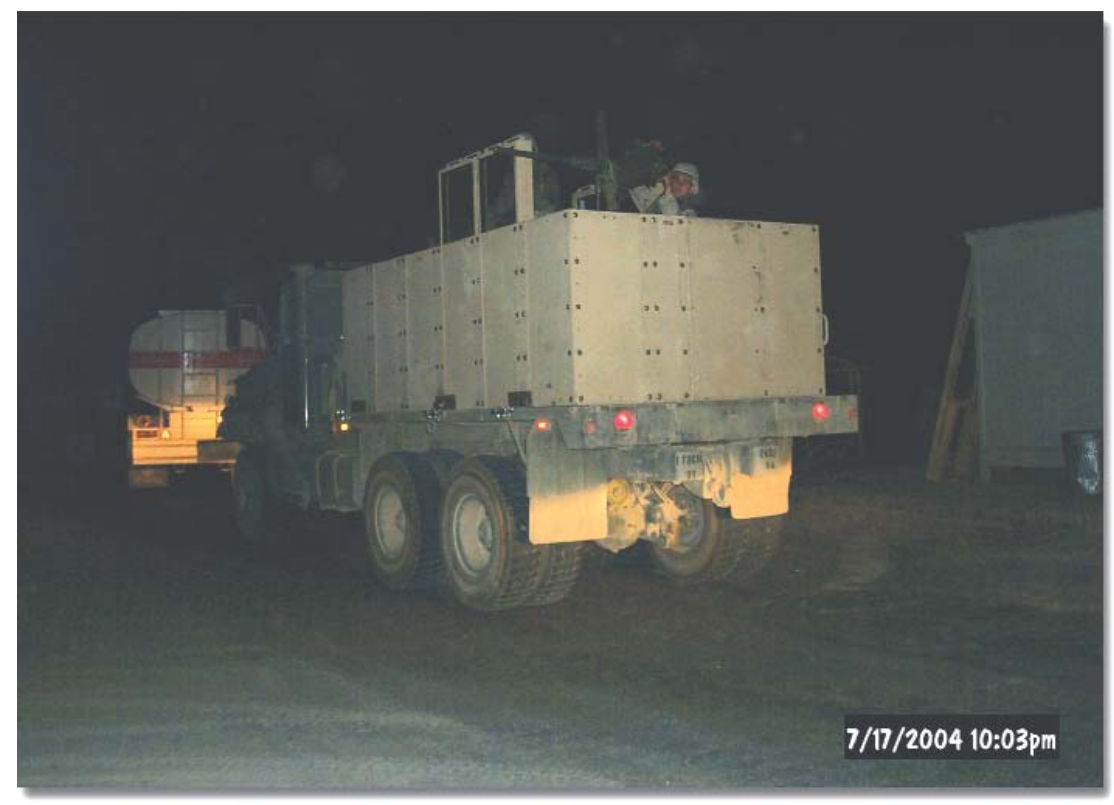

Figure 2. An M939 series gun truck leaving Camp Anaconda, Balad, for its first convoy escort mission with the U. S. Army 7th Transportation Battalion.

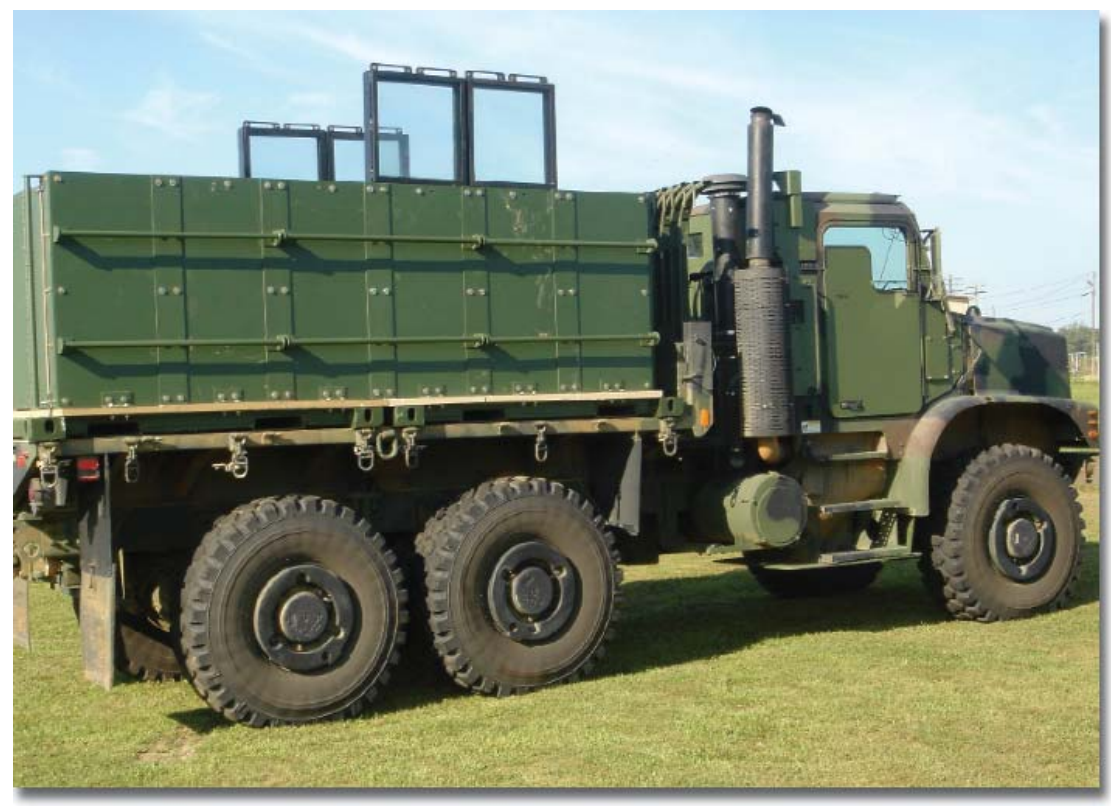

Figure 3. The USMC MTVR gun truck at ATC. 


\section{Experimental Verification of Correlation-Based Wavefront Sensing}

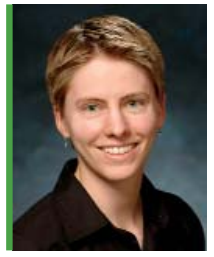

For more information contact Lisa A. Poyneer (925) 423-3360, poyneer1@\|nl.gov

\section{Project Goals}

Our two major goals are: to demonstrate experimentally that Correlation provides better performance than centroiding for the WFS, and to determine system requirements for $\mathrm{AO}$ operation for remote imaging, e.g., frame rates, light levels, and availability of scenes.

\section{Relevance to LLNL Mission}

AO remote imaging is important for national security applications. This project will further extend LLNL capabilities in this area. noise level. Second, Correlation can work with subimages of an extended target, greatly broadening the range of scenarios in which $\mathrm{AO}$ systems can function. This enables the use of AO to correct aberrations such as those on a lightweight optic or atmospheric turbulence in a remote imaging system.

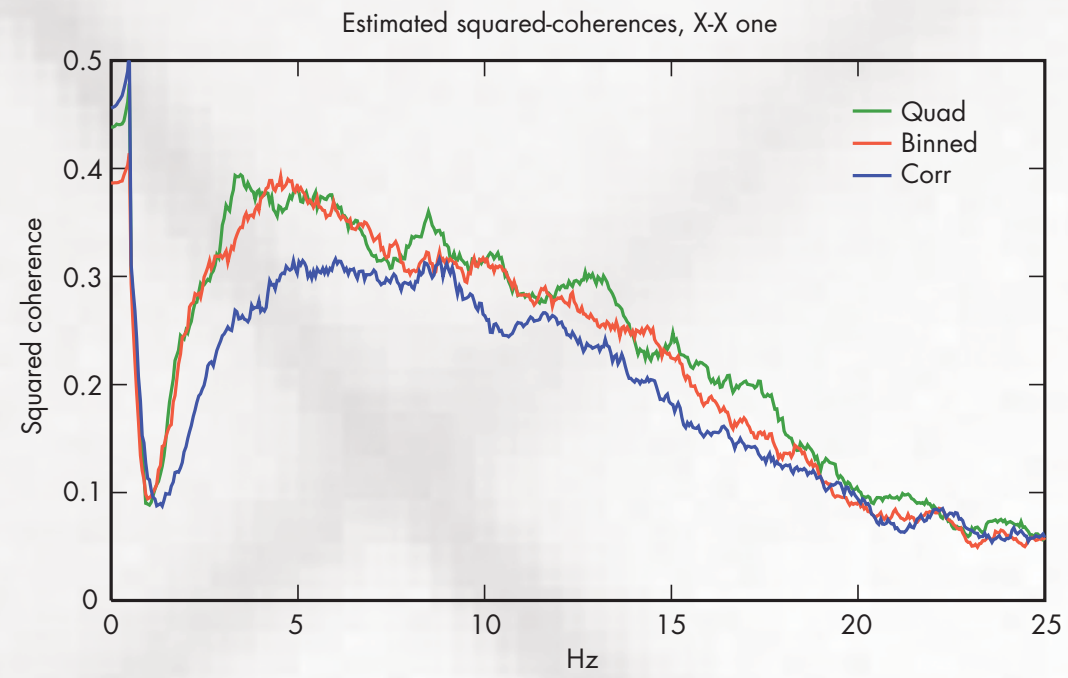

Figure 1. Temporal squared-coherence of slope measurements in closed-loop LGS operation of the Lick AO system.

\section{FY2004 Accomplishments and Results}

We have demonstrated for both the Lick Observatory Laser Guide Star (LGS) AO system and the Solid State Heat Capacity Laser (SSHCL) AO system that Correlation can improve performance.

For Lick, the Correlation algorithm provides more stable gain with equivalent noise to the current algorithms, enabling improved correction. Figure 1 shows temporal coherence between neighboring WFS measurements of the residual phase aberration in closed-loop operation. As the AO correction of the phase improves, neighboring measurements of the residual phase become less coherent. The Correlation algorithm produces better correction than the centroiding methods currently in use.

For SSHCL, the centroiding algorithm is significantly biased by deformations in spots on the WFS and background scatter. Correlation is unbiased, and as an extra benefit provides a three to four times reduction in mean-squared-error due to 
noise. Figure 2 shows the temporal power spectra in open-loop WFS measurements of the phase. Both algorithms measure the same amount of signal power at low temporal frequencies. However, Correlation has a much lower noise floor than centroiding, as is visible at higher frequencies.

For the remote imaging scenario, we use WFS subapertures of either 25- or 6.7-mm diameter to measure the phase slope.

Observing a variety of targets in sunlight, the slopes have no appreciable noise even down to exposures as short as $0.1 \mathrm{~ms}$. Using a point source, we characterized the temporal PSDs of the WFS slopes for short horizontal paths. The PSDs follow a negative power law, with slight variations in exact structure depending on conditions. We have measured the slopes using Correlation on scenes and have obtained the same PSD structure and total level of power. This is experimental evidence that we can use Correlation with a scene as the WFS method in this scenario.

We have characterized the atmospheric coherence length over many trials, with results for a $100-\mathrm{m}$ path clustering between 10 and $15 \mathrm{~mm}$. Our results indicate that if our subapertures or field of view are too large, anisoplanatism will cause small parts of the scene to be shifted in different directions. Since Correlation assumes the entire scene is shifted in the same direction, this will prevent the successful use of Correlation. A target scene which clearly demonstrates this phenomenon for the 25 -mm subaperture case is shown in Fig. 3.

\section{Related References}

1. Poyneer, L. A., "Scene-Based Shack-Hartmann Wavefront Sensing: Analysis and Simulation," Applied Optics, 42, pp. 5807-5815, October 2003. 2. Poyneer, L. A., K. La Fortune, and C. Chan,
"Scene-Based Wavefront Sensing for Remote Imaging," in SPIE 5162 Advanced Wavefront Control: Methods, Devices, and Applications, J. D. Gonglewski, M. A. Vorontsov, and M. T. Gruneisen, Eds., pp. 91-102, 2003.

\section{FY2005 Proposed Work}

We will continue our experimental work in the remote imaging scenario. We will use our experimental set-up to produce detailed system performance predictions for Correlation in comparison to established methods, such as speckle imaging and phase diversity. We will also experimentally verify the ability of Correlation to measure the aberrations on a lightweight nano-laminate optic.

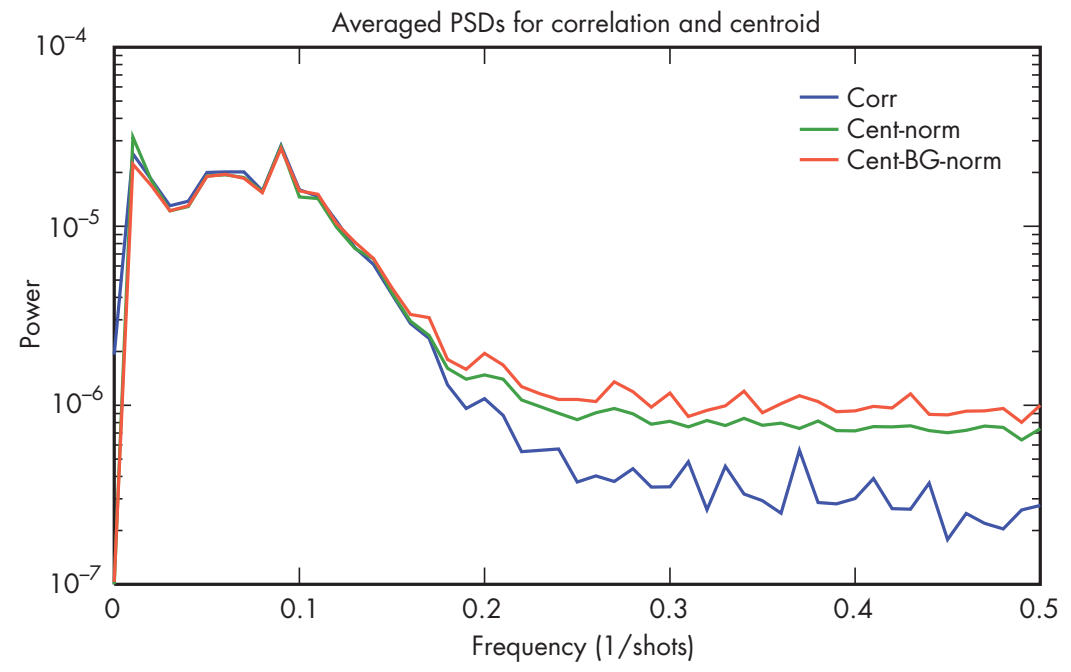

Figure 2. Temporal PSDs of open-loop WFS measurements for SSHCL.

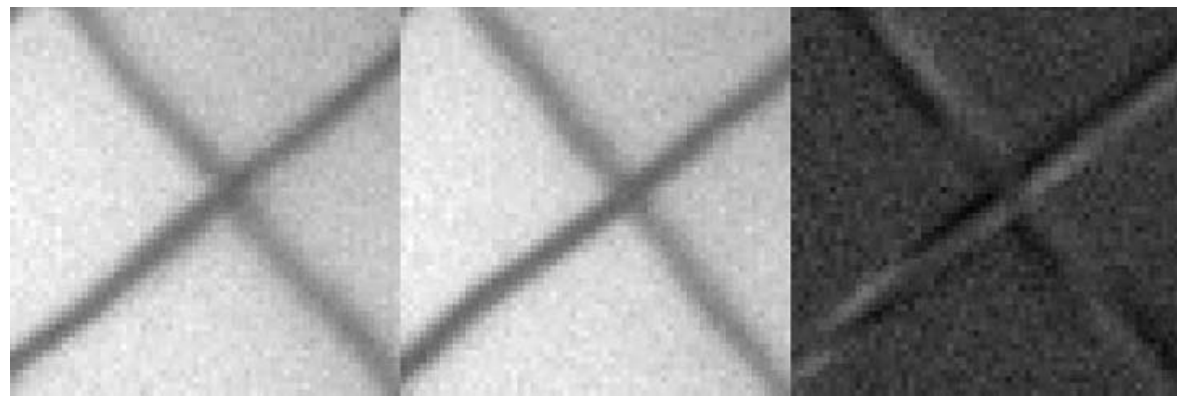

Figure 3. Sample scene demonstrating too-large subapertures and field of view. Shown are two sample subimages (a) and (b) from different points in time, and (c) the difference of these frames, clearly showing how different parts of the scene are deformed, as opposed to the entire scene shifted. 


\section{Real-Time Speckle Imaging for Video Surveillance}

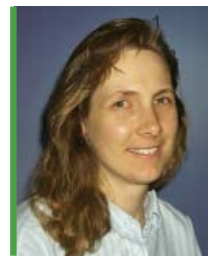

For more information contact Carmen J. Carrano (925) 422-9918, carrano2@|ln..gov

The objective of this new project is to test a real-time implementation strategy for high-resolution speckle-imaging systems.

\section{Project Goals}

The goals of the project for FY2004 were to continue to work on the user interface and speed up work on the speckleprocessing capabilities we focused on in the first year of the project. Specifically, we planned to 1) parallelize the speckleprocessing software over the four processors of our Quad Xeon server, with expected processing times under $20 \mathrm{~s}$ for many cases; and 2) perform field testing of the system with static and slowly moving targets.
Figure 1. Screenshot of user interface showing main control window (left) and speckle-processing parameters window (right). A sample interactive region of interest selected from the upper center image is shown in the lower center image loaded. magnitude or more. In previous work we have demonstrated that we can overcome this loss and restore the resolution to near the diffraction limit, making it possible to identify people from several kilometers and vehicles from multiple tens of kilometers. The original prototype system acquired and processed data far too slowly for most real-world applications, requiring the target to remain still for 2 to 3 min during data acquisition, and then wait 10 to $30 \mathrm{~min}$ or more for a processed image.

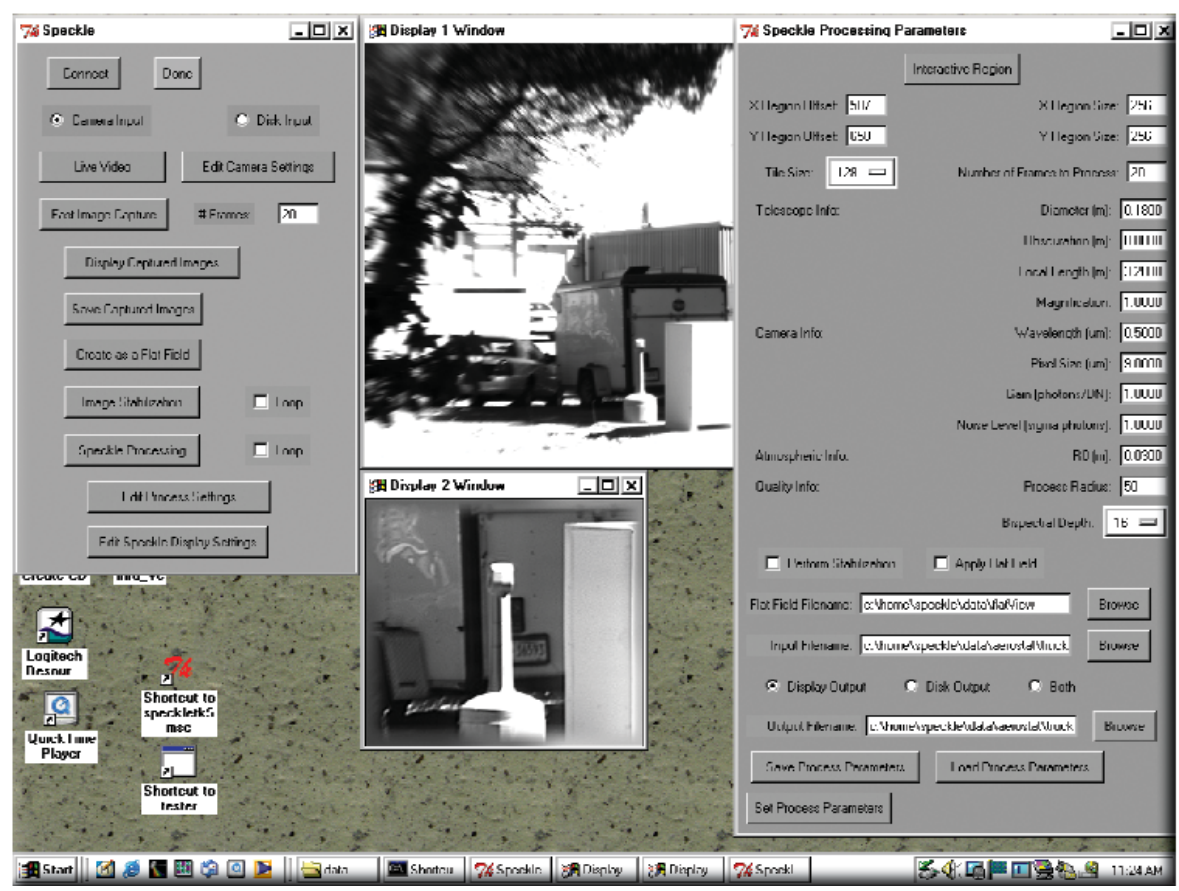

\section{Relevance to LLNL Mission}

This high-resolution imaging capability is potentially very important for the intelligence and DoD communities as well as for law enforcement and security agencies. LLNL is continuing to play a key role in the development of this capability.

\section{FY2004 Accomplishments and Results}

Over the course of this project, we have accomplished our stated goals and more. We have achieved successful videorate image data acquisition and display using a 2-mega-pixel CCD camera (1600x-1200 10-bit pixels) and a camera-link frame-grabber with a 512-MB buffer; converted all speckle software to $\mathrm{C}$; and performed parallelization using threads. For image sizes of $1024 \times 1024$ pixels or greater, we observed a three-times speedup in the total processing time using all four processors of the Quad Xeon server. We achieved processing times of $20 \mathrm{~s}$ for 30 frames of 1024-x-1024-pixel-sized images, and $5 \mathrm{~s}$ for 30 frames of 512-x512-pixel-sized images, out to the highest resolution and using image quality parameters that resulted in minimal artifacts. 
This year we implemented a functional user interface (Fig. 1), including cross platform compatibility with Linux and Windows operating systems, and the ability to perform interactive region-of-interest selection for the speckle processing. We have also produced a battery operable laptop version for remote experiments.

We have conducted successful experimental field-testing of the Linux server system at Site 300 and of the laptop system on Mt. Diablo. Figure 2 shows a sample result. Figure 3 is a photograph of the real-time video-surveillance system being tested outdoors.

Partnering with DOE, we enhanced our imaging project to achieve its science goals of imaging moving targets.

Finally, in FY2004, we defined two potential paths to video-rate processing, one using a DSP board architecture and the other using a compute cluster with high-speed interconnects.

\section{Related References}

1. Carrano, C. J., "Speckle Imaging Over Horizontal Paths," Proceedings of the SPIE-High Resolution Wavefront Control: Methods, Devices, and Applications IV, 4825, pp. 109-120, 2002.

2. Carrano, C. J., and J. M. Brase, "Horizontal and Slant Path Surveillance with Speckle Imaging," AMOS Technical Conference Proceedings, 2002.

3. Carrano, C. J., "Progress In Horizontal and SlantPath Imaging Using Speckle Imaging," Proceedings of the SPIE-LASE2003 Optical Engineering, 5001, pp. 56-64, 2003.

4. Carrano, C. J., and J. M. Brase, "Adapting HighResolution Speckle Imaging to Moving Targets and Platforms," SPIE Defense and Security Symposium, 5409, 2004.
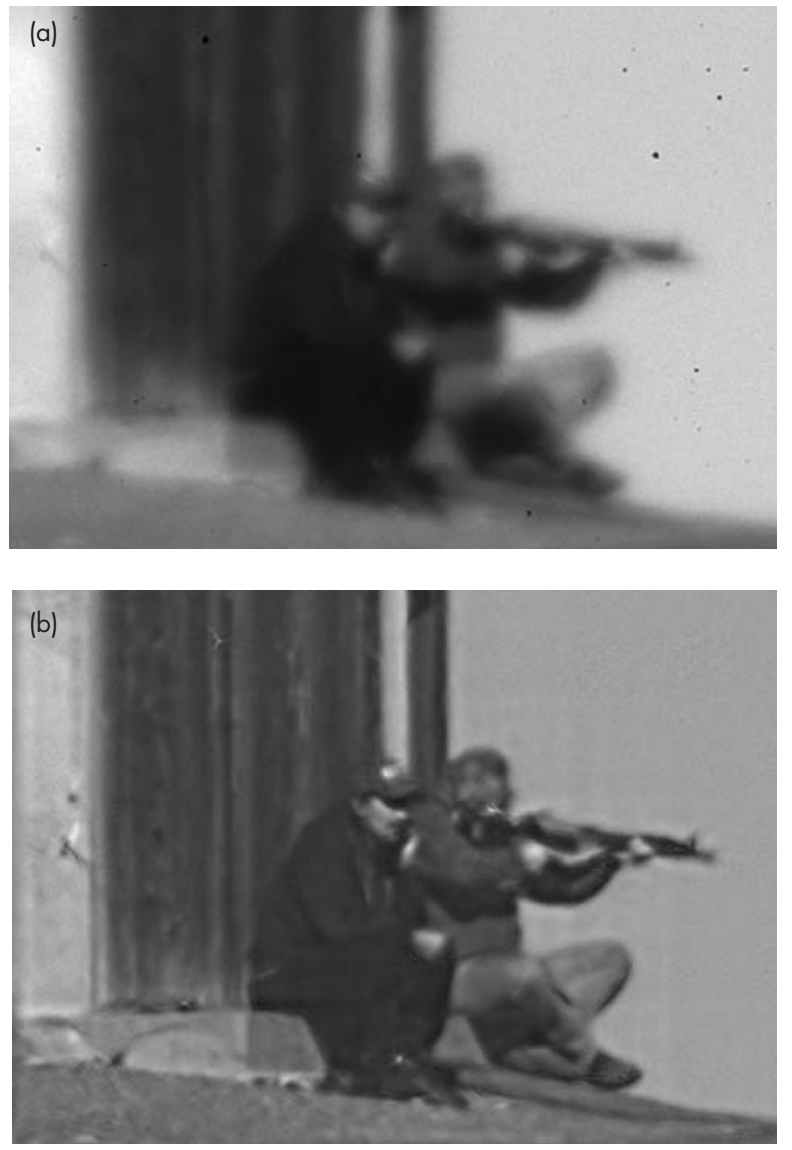

Figure 2. Dennis Silva and Jack Tucker from $2.7 \mathrm{~km}$ range at Site 300 through an 8-in. telescope. a) Sample raw unprocessed frame. b) Speckle-processed image from 40 raw frames.

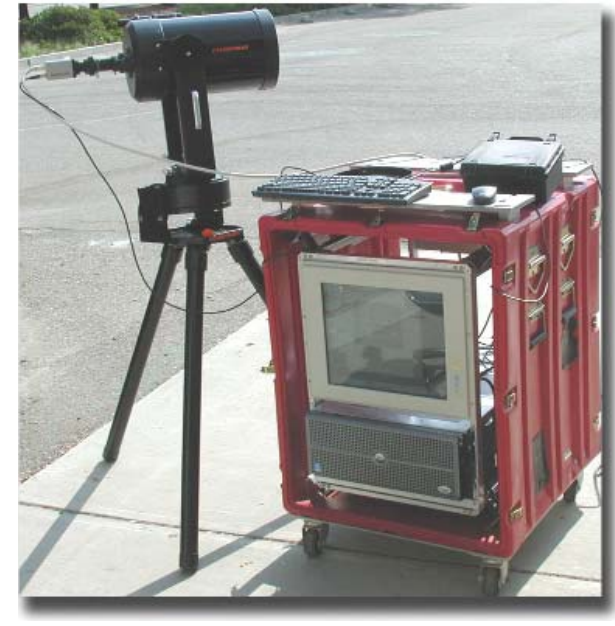

Figure 3. Photograph of the real-time video-surveillance system being tested outdoors.

\section{FY2005 Proposed Work}

We plan to modify the high-resolution speckle-imaging algorithm flow to allow for continuous updating of the high-resolution output image with each new input image. The current version takes a block of $\mathrm{N}$ input images and creates a single output image. We will then implement the continuous-update speckle software on our new nine-node Opteron computing cluster with Infiniband interconnects for the purpose of running at video rates. 


\section{Field-Programmable-Gate-Array-Based System for Network Operations}

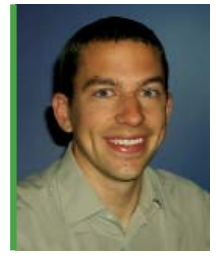

For more information contact J. Steven Blessing (925) 422-6680, blessing1@\|nl.gov

processing for various custom network applications, while giving software programmers the flexibility to code in highlevel languages.

Our prototype system comprises a number of individual intellectual property (IP) cores integrated in a single Xilinx V2P7 FPGA. These IP core system components included various networking hardware modules for handling packet filtering and physical network access, glue logic for integrating our components with the onboard PowerPC microprocessor, and bus and interconnect logic for placing all of the components on the single FPGA platform. In addition, we needed to write software for proof-of-concept that could identify packets of various protocol types.

\section{Relevance to LLNL Mission}

The major advantage to our work is high-performance network processing

which could achieve near wire-speed

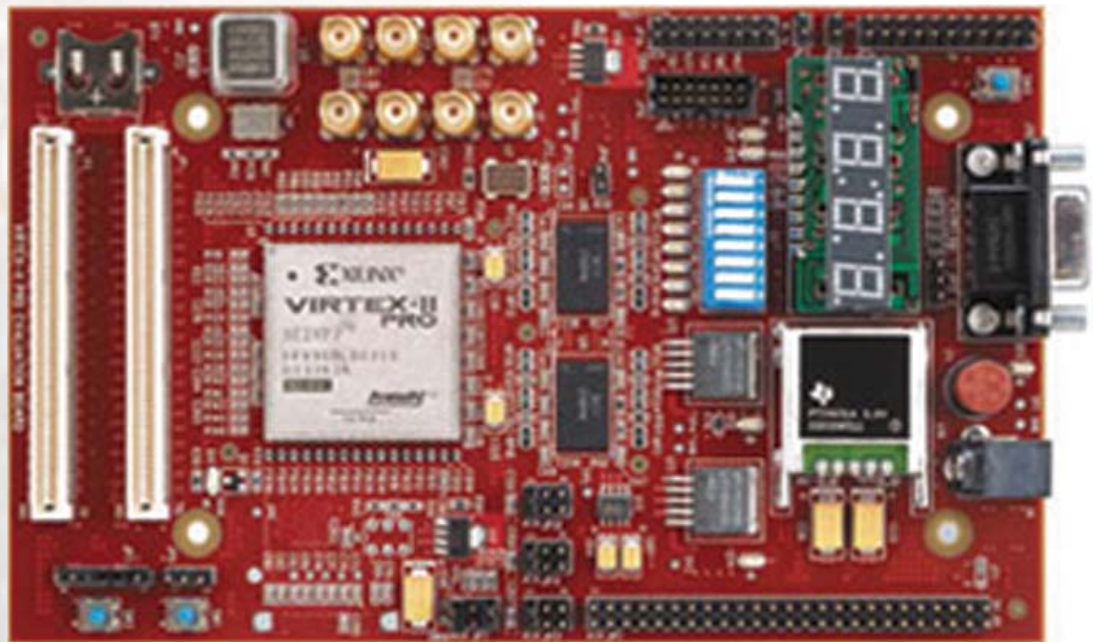

Figure 1. Avnet Virtex 2 Pro board used for prototype. 
with a very small system footprint. Such a system could serve as a basis for network analysis applications, or as an autonomous information sensor for distributed network monitoring operations such as firewalls or intrusion detection.

In addition, the individual components could be evolved into production-ready IP cores that are licensed outside of the Laboratory for other uses.

\section{FY2004 Accomplishments and Results}

Figures 1 and 2 illustrate our FY2004 effort.

To meet the needs of our packet filtering requirements, we evaluated a number of preliminary architectures. We examined several solutions involving hardware state machines, but eventually decided on a full network microprocessor, due to its flexibility and control.

Silverthrone is an IP core implementation of a network processor built to support the BSD Packet Filter language (BPF). The BPF language is used by many firewall filters for efficient packet classification and filtering. To our knowledge, however, it has only been implemented in software as a virtual machine, and never in actual hardware.

Silverthrone consists of a custom hardware microprocessor that fully implements the BPF instruction set with some additional extensions for packet manipulation. The system executes all BPF instructions in, at most, 3 clock cycles, and mirrors the BPF with a register-based packet filter that uses a directed acyclic control graph. This gives a significant performance increase over a standard expression tree mode, which may contain redundant operations. Our un-optimized prototype implementation of the processor yields clock speeds of approximately $90 \mathrm{MHz}$, which allows us to identify IP packets in 10 clock cycles. This allows Silverthrone to achieve a theoretical processing rate of 9 million packets per second, given a network front-end capable of writing data at this speed.

With a custom network processor, we also enable users to work with the existing BPF instruction set, allowing the direct use of our architecture without having to

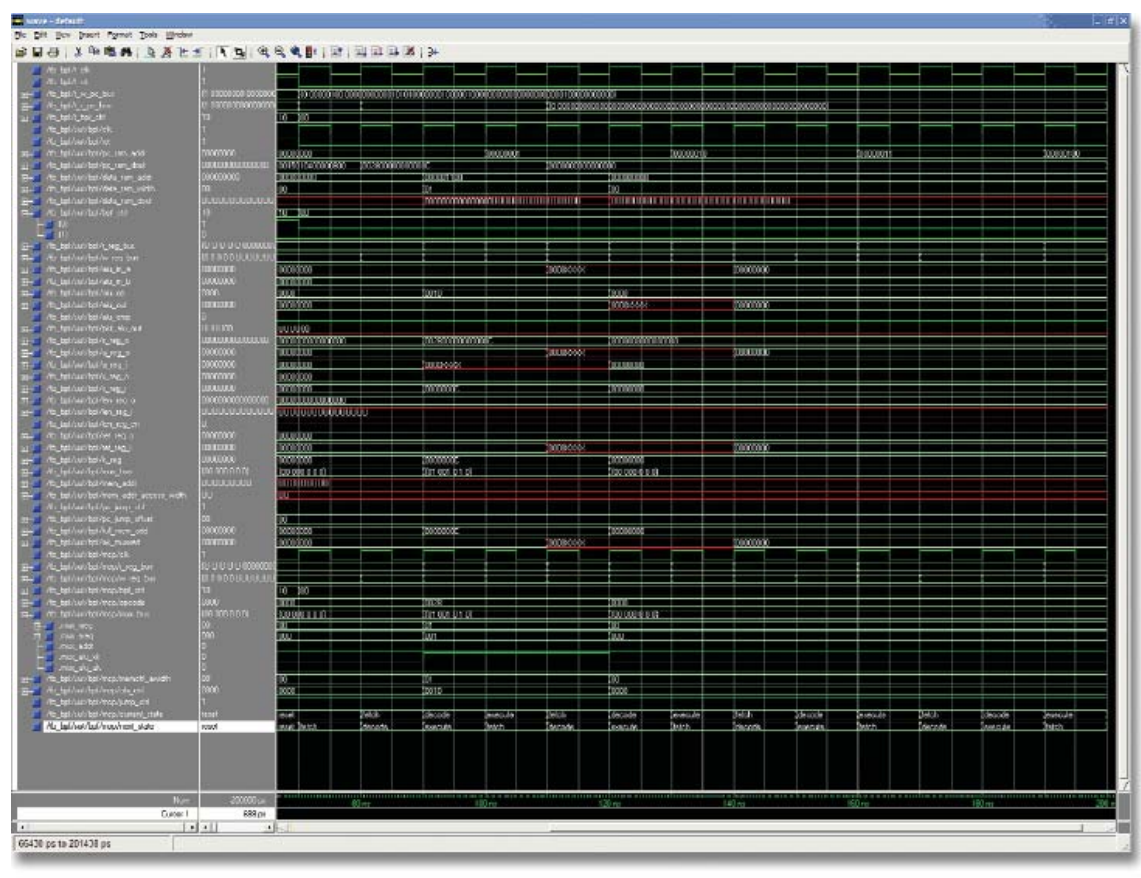

Figure 2. Signal capture of Silverthrone executing single packet instruction.

understand the underlying hardware. We have also preserved full compatibility with the existing software tools using the BPF instructions.

Silverthrone also contains extensions to the BPF instruction set to allow the network processor to perform more advanced packet analysis than is possible in traditional software based implementations.

\section{Related References}

1. Bloom, B., "Space/Time Trade-Offs in Hash Coding with Allowable Errors," ACM, 13, (7), pp. 422-426, May 1970.

2. Dharmapurikar, S., P. Krishnamurthy, T. Sproull, and J. Lockwood, "Deep Packet Inspection Using Parallel Bloom Filters," Hot Interconnects, pp. 4451, August 2003.

3. Ramakrishna, M., E. Fu, and E. Bahcekapili, “A Performance Study of Hashing Functions for

Hardware Applications," Proc. ICCI, pp. 1621-1636, 1994.

4. Ji, H. M., and M. Carchia, "Fast IP Packet Classification with Configurable Processor," IEEE, pp. 2268-2274, 2001.

5. McCanne, S., and V. Jacobson, "The BSD Packet Filter: A New Architecture for User-level Packet Capture," USENIX Conference Proceedings, 1993.

\section{FY2005 Proposed Work}

We envision extending the existing Silverthrone platform to include analysisspecific operations. These could include: text processing, dynamic session parsing, and stateful packet inspection. The Silverthrone platform can also be extended to provide wireless capabilities such as network mapping and vulnerability assessment. 


\section{Flexible Testbed for Evaluation of Ultra-Wideband (UWB) Radios}

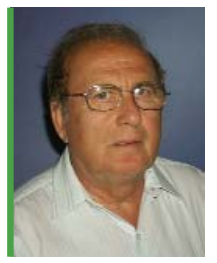

For more information contact Alex Spiridon (925) 423-3368, spiridon1@|ln..gov

evaluate UWB link capacity and robustness against fades and interference relative to that of narrowband communication.

The approach is to combine measured RF signals and interference with computer emulation of receiver processing to test options, and provide fidelity, flexibility and quick reaction. The technical challenge is to make the system usable for realistic rapid prototyping, and to control bandwidth and performance. Results will expand and validate initial UWB link and receiver models.

By the end of the project the following are to be accomplished: quantify and identify the advantages of bandwidth, and demonstrate the array gain possible with spatial processing; compare UWB to legacy narrowband links; compare LLNL's version to other UWB configurations; identify enhancements to LLNL UWB; and provide a user-friendly interface.

The flexible testbed will provide a systematic method and capability to evaluate link performance. This establishes a kernel of a library of models that will be extended as additional tasks are performed. In addition, the tool will allow a more comprehensive and realistic setting to optimize and evolve the UWB system. The effort potentially offers opportunities in the following areas: incorporation of supercomputers into the testbed; systematic optimization of the link system; and evolving hybrid simulation.

Figure 1. UWB testbed Tx and Rx block diagram. 


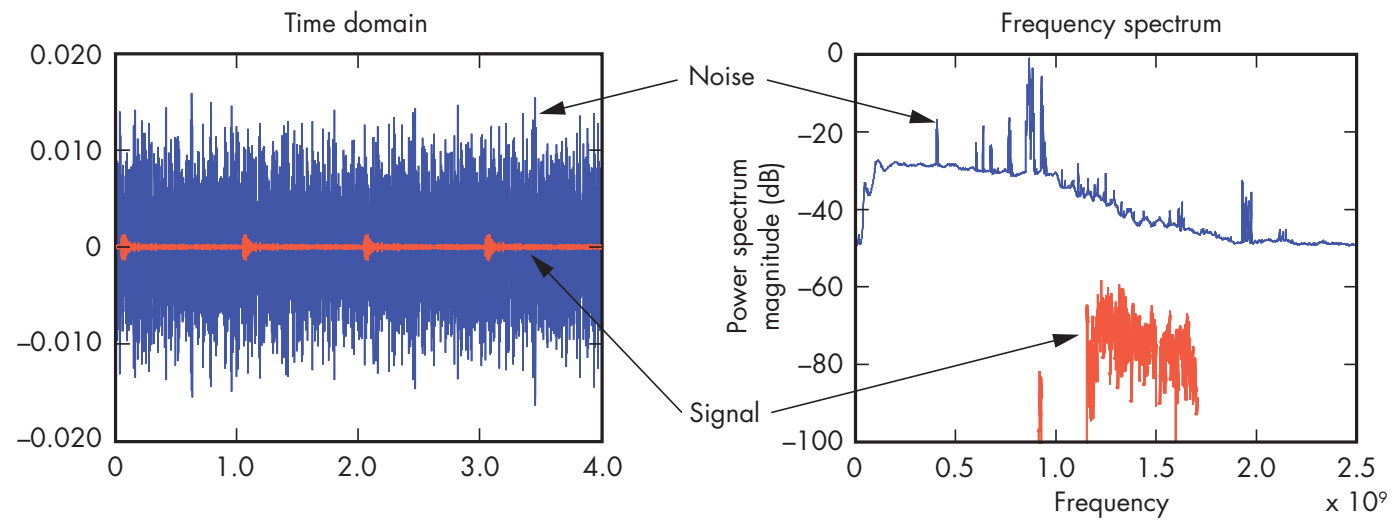

Figure 2. Illustrative results: at $250 \mathrm{~m}$ away, a 5-mW UWB signal was received and decoded by our novel feedback loop system.

\section{Relevance to LLNL Mission}

LLNL internal programs such as NIF, customer applications in LUGA, and homeland defense, have a need and an interest in wireless LAN that is robust against fades and interference, and that has low signature, reducing the vulnerability to intercept and detection.

\section{FY2004 Accomplishments and Results}

We have implemented an untethered testbed, intended to allow data, in the form of raw bits, files, or streaming information, to be turned into UWB bursts and transmitted at varying bit rates. These UWB RF bursts are then picked up and saved by a synchronized receiving system. The receiver uses a high-speed real-time sampling oscilloscope, taking samples of the RF at $5 \mathrm{Gs} / \mathrm{s}$ and saving that information. The transmitter and receiver are not tethered to each other in any way. They rely solely on GPS timing to remain synchronized with each other. This allows the system to be deployed over great distances without the need for a cable synchronization tether connecting the two units. Basic system block diagrams of the Rx and Tx units are shown in Fig. 1.
In FY2004, we also coarsely estimated path loss and fades; collaborated with models; and introduced two IP single-pulse modulation and channel-matched signaling. Example results are shown in Fig. 2.

\section{Related References}

1. Nekoogar, F., F. Dowla, and A. Spiridon, "SelfOrganization of Wireless Sensor Networks Using Ultra-Wideband Radios," IEEE Radio and Wireless Conference, Atlanta, Georgia, September 19-22, 2004.

2. Dowla, F., F. Nekoogar, and A. Spiridon,

"Interference Mitigation in Transmitted-Reference Ultra-Wideband Receivers," IEEE International Symposium on Antennas and Propagation, Monterey, California, June 20-26, 2004.

3. Nekoogar, F., F. Dowla, and A. Spiridon, "Rapid Synchronization of Ultra-Wideband TransmittedReference Receivers," Sixteenth International Conference on Wireless Communications, Calgary, Canada, July 12-14, 2004.

4. Nekoogar, F., F. Dowla, and A. Spiridon, “A Novel Covert Transmitted-Reference Ultra-Wideband Communications," Military Communications Conference, Monterey, California, October 31November 3, 2004.

5. Spiridon, A., F. Dowla, F. Nekoogar, and D. Benzel, "On the Performance of Ultra-Wideband TransmittedReference Receivers," IEEE Trans. Comm., 2004.

\section{FY2005 Proposed Work}

We plan to add a GUI to the system and tabulate procedures; evaluate spatial processing (such as omni vs. directional, and two antennae vs. one); increase pulsing from $1 \mathrm{Mps}$ to $20 \mathrm{Mps}$, and $\mathrm{BW}$ from $0.2 \mathrm{GHz}$ to $2 \mathrm{GHz}$ (improving confidence and statistics); correlate data with theoretical models; and evaluate and analyze stressed links and two IPs. 


\section{Laser Peening Study for Reduction in Hydrogen Permeation in a Titanium Alloy}

Initial
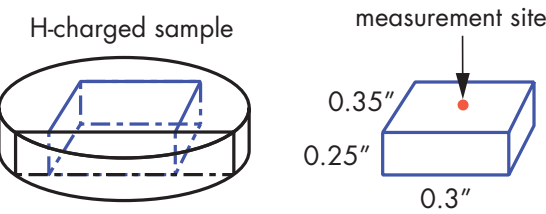

Cut coupon for SIMS measurement

Figure 1. Initial hydrogen-charged coupon and SIMS measurement location.

(a) NIST standard reference material 352c

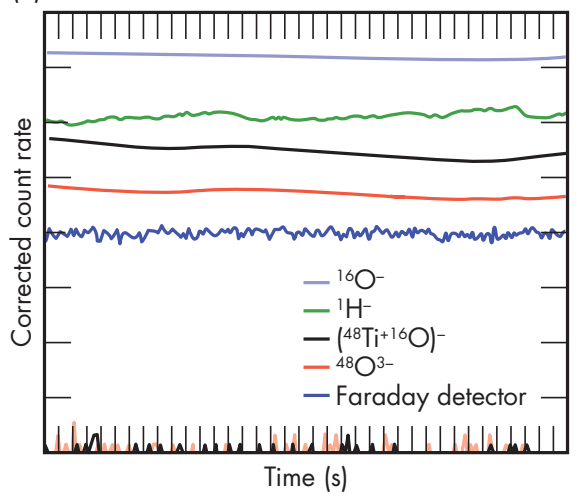

Figure 2. NIST Standard reference material (a) and our sample (b).

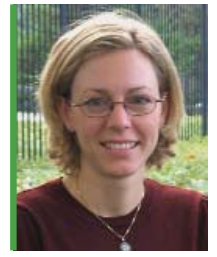

For more information contact Tania Zaleski (925) 423-7493, zaleski2@|ln.gov

types of systems. Equipment components used in the production of these hydrogen isotopes often have finite service lives due to degradation by hydrogen penetration. Extending the lifetime would potentially have significant cost savings as well as implications for higher performance. Hydrogen embrittlement also will have significant impact as the United States moves to a hydrogen-based fuel economy. This process could potentially improve fuel cell performance and could allow for safer and denser storage of hydrogen.

\section{Relevance to LLNL Mission}

Of particular interest to the Laboratory and DOE is the potential benefit of this study for applications in deuterium and tritium production and storage. The process could be used to extend the life of components that store hydrogen and its isotopes, and to a broad range of metals subjected to corrosive environments. Such critical parts include gas storage vessels for nuclear weapons, the tools and machines used to form these components, equipment used to produce the gas, and nuclear storage containers.

\section{FY2004 Accomplishments and Results}

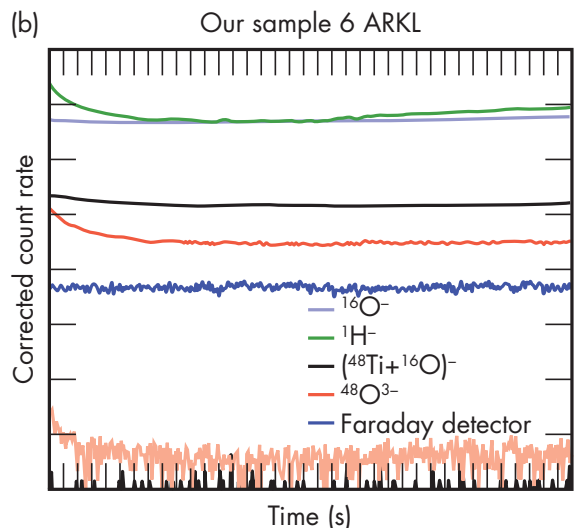

For our material preparation and hydrogen detection work, we tested Ti$6 \mathrm{Al}-4 \mathrm{~V}$ double phase. One coupon was laser peened with $10 \mathrm{GW} / \mathrm{cm}^{2}, 18-\mathrm{ns}$ pulse width, and two layers of peening.

Cathode charging was performed on the as-received and laser-peened specimens. Each specimen was fully immersed in a vessel of a four-port electrochemical cell containing an aqueous electrolytic charging solution of $5 \% \mathrm{H}_{2} \mathrm{SO}_{4}$ at ambient temperature. A precision power supply was connected by clip leads to the three electrodes and provided a constant, uniform exchange 
current density of $10 \mathrm{~mA} / \mathrm{cm}^{2}$ onto the specimen, over a charging duration of $144 \mathrm{~h}$.

The parameters for the secondary ion mass spectroscopy (SIMS) measurement are as follows: a pattern $250 \mu \mathrm{m} \times$ $250 \mu \mathrm{m}$ in size was rastered across the surface of the coupon, to a depth of $85 \mu \mathrm{m}$. Figure 1 shows the coupon dimensions and initial measurement location. The ions collected from this trace were compared with a standard reference material $(352 \mathrm{c})$ containing hydrogen concentration of $49.0 \pm 0.9 \mu \mathrm{g} / \mathrm{g}$ in unalloyed titanium. The trace made on the NIST standard material was assumed to be homogeneous (see Fig. 2).

Four main ions were recorded in each sample, ${ }^{16} \mathrm{O}^{-},{ }^{1} \mathrm{H}^{1-},\left({ }^{48} \mathrm{Ti}^{+16} \mathrm{O}\right)^{-}$, and $\mathrm{O}^{3}$. The scans of each coupon showed very consistent levels of each ion to the depth measured. Results also show the counts of each ion to be very constant over each scanned volume. This means the material is fairly uniform in the region measured, allowing a fair comparison of each ion concentration between the two samples.

The data in Fig. 3 show the recorded ion counts for each ion measured. When compared to the standard, our sample showed almost ten times the ratio of $\mathrm{H}^{1-}$ ions as compared to the standard, or roughly $490 \mu \mathrm{g} / \mathrm{g}$. This demonstrates that we can measure the hydrogen content in our Ti-6Al-4V material and quantify its concentration. Since we want to compare the hydrogen embedding depth in LP versus non-LP samples, our next step is to measure three coupons with various treatments using a step scan method.

\section{Related References}

1. Peyre, P., C. Braham, J. Ledion, L. Berthe, and R. Fabbro, Journal of Materials Engineering and
Performance, 9, pp. 656-662, 2000.

2. Peyre, P., X. Scherpereel, L. Berthe, C. Carboni, R. Fabbro, G. Beranger, and C. Lemaitre, Material Science and Engineering, A280, pp. 294-302, 2000 3. Iost, A., and J. B. Vogt, Scripta Metallurgical et Materialia, 37, pp. 1499-1504, 1997.

\section{FY2005 Proposed Work}

We would like to investigate the hydrogen charging conditions to optimize the parameters for hydrogen penetration. Investigating different cathode charging conditions, such as current density and charging time, would allow for a more accurate comparison of hydrogen penetration environments.
NIST Standard with 49.0pg/g Hydrogen Concentration

Block data for DR4722A.002 (SRM 352c initial primary DVM reading: 0.27862) Raster ON
Figure 3. Measurement results for ion concentration.

Block data parameters:

Using $\mathrm{Ti}^{+} \mathrm{O}$ as reference

Starting cycle: 1 Ending cycle: 190 Cycles per block: 190

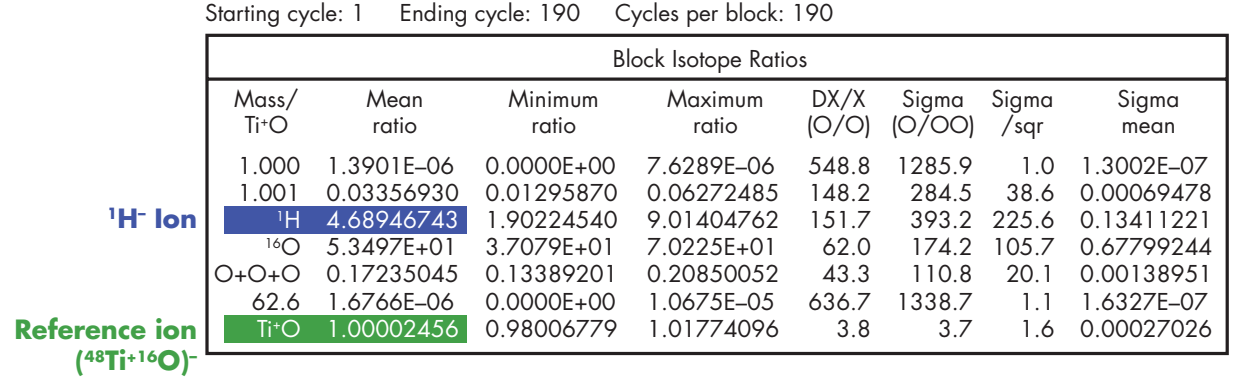

H-Charged Sample

Block data for DR4723A.001 (6 AR KL laser-peen initial primary DVM reading: 0.27763) Raster ON, $10 \mathrm{E}+10$ ohm on FC

Block data parameters:

Using $\mathrm{Ti}^{+} \mathrm{O}$ as reference

Starting cycle: 1 Ending cycle: 500 Cycles per block: 500

\begin{tabular}{|c|c|c|c|c|c|c|c|c|}
\hline & \multicolumn{8}{|c|}{ Cycles per block: 500} \\
\hline & \multicolumn{8}{|c|}{ Block Isotope Ratios } \\
\hline & $\begin{array}{c}\text { Mass/ } \\
\mathrm{Ti}^{+} \mathrm{O}\end{array}$ & $\begin{array}{l}\text { Mean } \\
\text { ratio }\end{array}$ & $\begin{array}{l}\text { Minimum } \\
\text { ratio }\end{array}$ & $\begin{array}{l}\text { Maximum } \\
\text { ratio }\end{array}$ & $\begin{array}{l}D X / X \\
(O / O)\end{array}$ & $\begin{array}{l}\text { Sigma } \\
(O / \bigcirc \bigcirc)\end{array}$ & $\begin{array}{l}\text { Sigma } \\
\text { /sqr }\end{array}$ & $\begin{array}{l}\text { Sigma } \\
\text { mean }\end{array}$ \\
\hline \multirow{5}{*}{${ }^{1} \mathrm{H}^{-}$Ion } & $\begin{array}{l}1.000 \\
1.001\end{array}$ & $\begin{array}{l}2.9703 E-06 \\
0.03082788\end{array}$ & $\begin{array}{l}0.0000 E+00 \\
0.01911497\end{array}$ & $\begin{array}{l}2.8516 E-05 \\
0.04381545\end{array}$ & $\begin{array}{r}960.1 \\
80.1\end{array}$ & $\begin{array}{r}1340.5 \\
146.1\end{array}$ & $\begin{array}{r}1.1 \\
13.8\end{array}$ & $\begin{array}{l}1.7824 \mathrm{E}-07 \\
0.00020156\end{array}$ \\
\hline & IH & $4.3765 \mathrm{E}+01$ & $3.3135 \mathrm{E}+01$ & $1.1504 \mathrm{E}+02$ & 187.5 & 230.0 & 124.5 & 0.44969863 \\
\hline & ${ }^{16} \mathrm{O}$ & $3.3581 \mathrm{E}+01$ & $2.3614 \mathrm{E}+01$ & $3.8100 E+01$ & 43.1 & 104.0 & 55.3 & 0.15639845 \\
\hline & $\mathrm{O}^{+} \mathrm{O}^{+} \mathrm{O}$ & 0.22053018 & 0.17884158 & 0.56418800 & 174.7 & 221.5 & 38.6 & 0.00218714 \\
\hline & 62.6 & 2.8683E-05 & $0.0000 E+00$ & $1.3040 \mathrm{E}-04$ & 454.6 & 524.6 & 1.3 & 6.7366E-07 \\
\hline Reference ion & $\mathrm{Ti}^{+} \mathrm{O}$ & 1.00002277 & 0.98597926 & 1.02062881 & 3.5 & 4.4 & 1.7 & 0.00019492 \\
\hline
\end{tabular}

$\left({ }^{48} \mathrm{Ti}^{+16} \mathrm{O}\right)^{-}$ 


\section{Hyperspectral Processing Using FPGAs and DSPs}

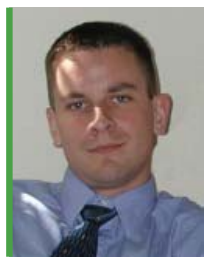

For more information contact Erik David Jones (925) 424-4757, jones157@|ln..gov

requirements, real-time processing systems will need to be assembled using combinations of FPGAs and DSP processors rather than bulky, expensive, and less efficient general purpose computing devices. Smaller instruments with real-time feedback will increase the portability, range and value for many of the Laboratory's customers.

\section{Project Goals}

Our goals are to evaluate the processing requirements to perform covariance estimation, eigenfactorization, and matched filtering (L3 processing) hyperspectral data processing; to build a controller to archive data and operate the A/D and DSP components using a single board computer; to build a prototype to digitize at least four channels of analog information clocked off a hyperspectral focalplane array, and process the data prior to L3 by performing bad-pixel correction and spectral calibration (L1 processing); build a prototype that performs real-time hyperspectral data processing from a complete data cube, through one or more algorithms selected from the L1 and L3 processing chains (Fig. 1).

\section{Relevance to LLNL Mission}

This project will provide the Laboratory with engineering experience in small-scale, low-power, low-cost, realtime processing systems for hyperspectral 
imagery that can be extended to any complex mathematical problem where P3 requirements are key.

\section{FY2004 Accomplishments and Results}

Our major milestones include the following: all algorithms have been identified and coded in C; covariance estimation, eigenfactorization, and L3 processing have been implemented; all hardware and software for engineering the final configuration have been acquired and tested (see Figs. 2 and 3 for examples); packaging has been assembled; and spectral calibration and L1 processing have been synthesized for the Xilinx Virtex II FPGA. Portions of those algorithms have been tested and are being assembled for system verification and performance metrics.

\section{Related References}

1. Turke T., and V. Lohweg, "A Real-Time ImageProcessing-System-on-Chip for Security Feature Detection and Classification," Proceedings of Spiethe International Society for Optical Engineering, 5297, (1), pp. 204-11, 2004.

2. Hinnrichs, M., and B. Piatek, "Hand-Held Hyperspectral Imager for Chemical/Biological and Environmental Applications," Proceedings of Spiethe International Society for Optical Engineering, 5270, (1), pp. 10-18, 2003.

3. Batlle J., J. Marti, P. Ridao, and J. Amat, "A New FPGA/DSP-Based Parallel Architecture for Real-

Time Image Processing," Real-Time Imaging, 8, (5), pp. 345-56, Academic Press, UK, October 2002.

4. Jaenick, A., and W. Luk, "Parameterised FloatingPoint Arithmetic on FPGAs," IEEE International Conference on Acoustics, Speech, and Signal Processing Proceedings, 2001.

5. Ho, C. H., et al., "Rapid Prototyping of FPGABased Floating Point DSP Systems," Proceedings of IEEE International Workshop on Rapid System Prototyping, 2002.

\section{FY2005 Proposed Work}

While the DSP board can perform L3 processing and the $A / D$ board can digitize and perform L1 processing, the data path between the boards is marginal. Additionally, the $\mathrm{L} 3$ processing must be optimized. The covariance estimation is particularly intensive due to the number of memory read/writes. Therefore, the FPGA on the DSP board will be used to either calculate the covariance or provide a buffer/pre-fetch area to mitigate the poor performance reading/writing from memory. We will optimize the L3 processing and the data pathways from analog signal to bytes on disk.

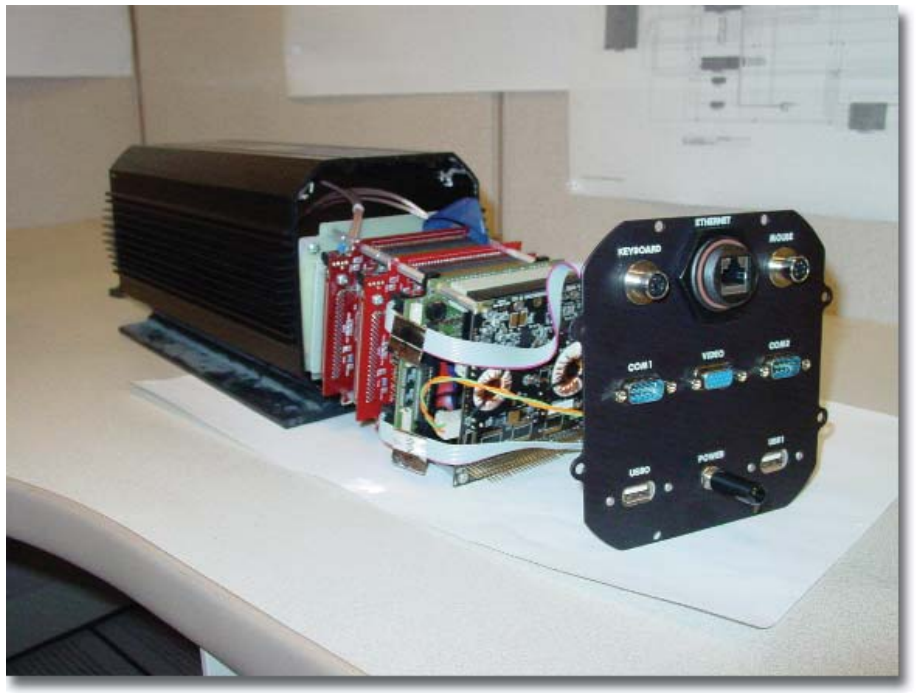

Figure 2. Hardware implementation for hyperspectral embedded processing system.
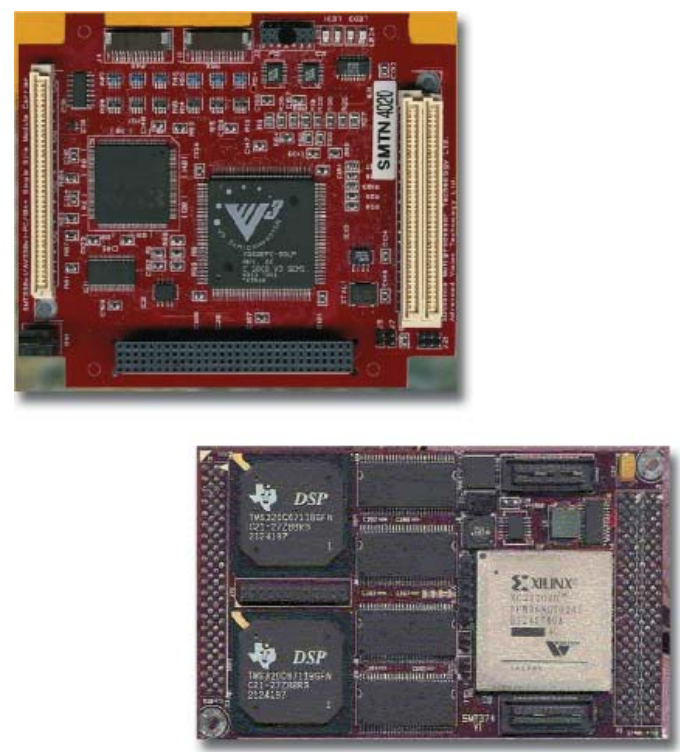

Figure 3. Sundance PC-104+ carrier board (top) and Sundance dual TI 6713 DSP module (bottom). 


\section{Magnetic Matching of Ultra-Compact Marx Generator}

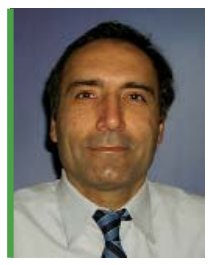

For more information contact Jay B. Javedani (925) 422-8569, javedani1@|lnl.gov

LNL has produced a family of ultracompact Marx-type high-voltage generators (UCMG) for a variety of special pulsed-power applications, such as drivers for compact x-ray sources and highpower RF sources.

The UCMG incorporates low-profile, low-inductance capacitors and switches, high-energy-density ceramic materials, high-electrical-strength dielectric materials, optimally shaped parts, and integrated packaging techniques to achieve higher performance in relatively small packages. As with any pulsed-power source, energy transfer can be improved by matching the impedance of the driver to the load. Load matching can be achieved by saturable reactance ferrites, to provide a nonlinear transition from high to low impedance during the rise time of the UCMG output current.

\section{Project Goals}

The primary goal of this project was to perform a proof-of-concept demonstration of the magnetic matching of the UCMG. An underlying objective was to explore the basic properties and response of ferromagnetic materials under very high electrical stress and fast-pulsed conditions. A further objective was to create representative core models for circuit modeling of the UCMG for particular applications. (a)

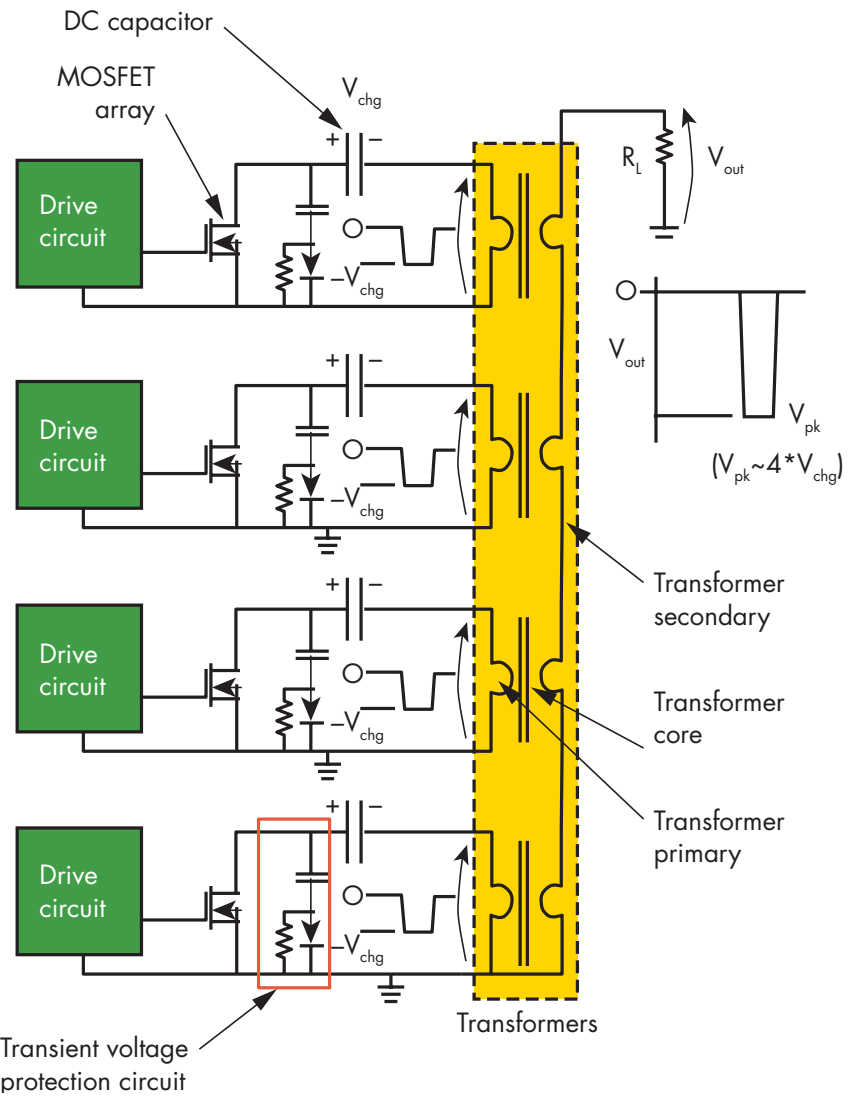

(b)

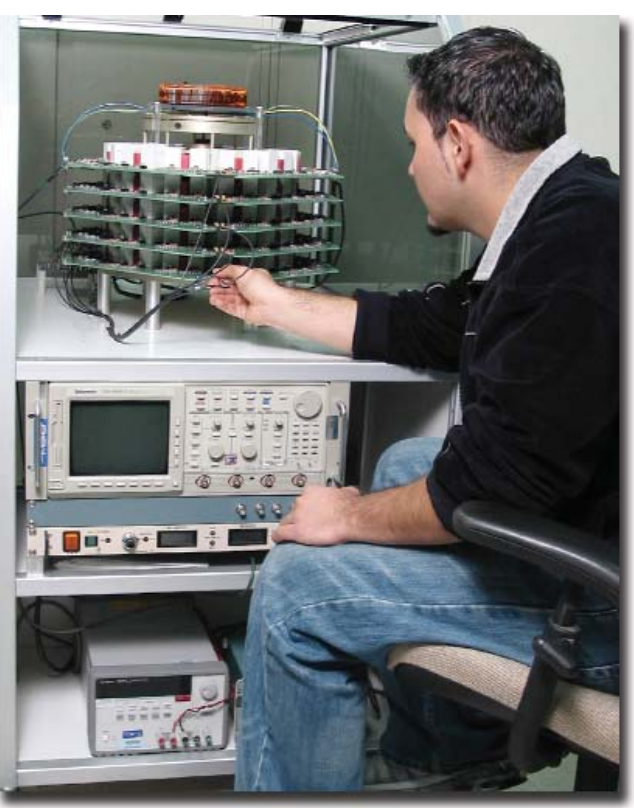

Figure 1. (a) Schematic and (b) photo of five-stage 3-kV pulser assembled for core testing. 


\section{Relevance to LLNL Mission}

The UCMG is the enabling technology for a new high-performance flash x-ray source for subcritical experiments that are performed in underground alcoves at NTS. This source can be improved by using ferrite materials to suppress prepulse, and by providing better matching to the rod-pinched $\mathrm{x}$-ray diode. The UCMG is also useful for other pulsed-power applications of interest to DNT, NAI, and other government agencies.

\section{FY2004 Accomplishments and Results}

We successfully completed the tasks we set for FY2004.

We chose CMD5005 from Ceramic Magnetics as the material to benchmark in our experiments. The manufacturer's specifications for CMD5005 are as follows:

- initial permeability: 1600

- maximum permeability: 4500 typ

- maximum flux density: 0.3 Tesla min

- remnant flux density: 0.18 Tesla max

- coercive force: 0.23 Oe max.
The B-H curves were derived using both $\mathrm{AC}$ and pulsed sources. The AC testing was done with a variable frequency power source operated from $60 \mathrm{~Hz}$ to $5 \mathrm{kHz}$. Results from the AC testing were in good agreement with the manufacturer's claims.

For the fast-pulse testing, we adapted hardware from beam kicker applications. A five-stage, 3-kV pulser, shown in Fig. 1, was assembled and operated to survey small toroidal cores. To test larger cores we used an existing 70-stage, $50-\mathrm{kV}$ pulser. Both pulsers produce output pulses with rise times of several nanoseconds and pulse widths up to a microsecond, depending on the load.

In an alternate scheme, the smaller cores were also tested with a single-stage UCMG. In this configuration, the cores are placed around current return rods alongside the generator, as shown in Fig. 2. Excitation current was measured using current viewing resistors at the base of each return rod. Output voltage from a single turn secondary winding around a single core was monitored with differential high-voltage probes. A typical B-H curve derived using this technique is also shown in Fig. 2, as are the effects of adding multiple cores per return rod, increasing the output voltage in proportion to the inductance, until the cores saturate.

For the proof-of-concept demonstration, ferrite cores were added to current return rods around a 10-stage UCMG. Better impedance matching results in higher output power. In the optimized case of 5-x-6 cores, the peak output power is about $2.1 \times 10^{9} \mathrm{~W}$, which is about $50 \%$ greater than the no-core case.

Core models produced through our special measurements have been incorporated into circuit models that are being used to evaluate improvements in targeted applications.

\section{Related Reference}

Goerz, D. A., and M. Wilson, "Ultra-Compact MarxType High-Voltage Generator," U.S. Patent 6,060,791 issued May 9, 2000 (a)

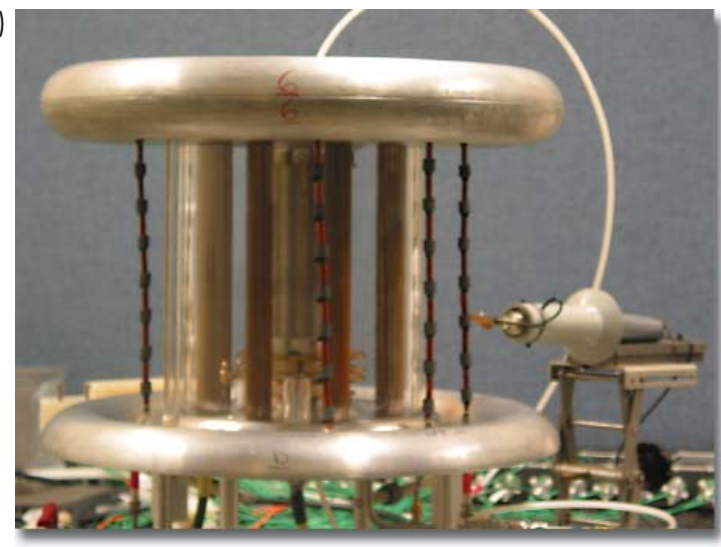

(b)

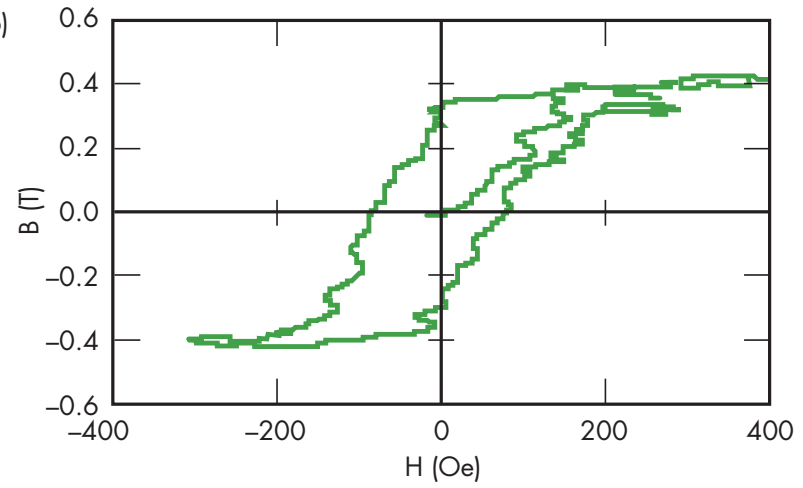

Figure 2. (a) Photo of single-stage UCMG with seven cores per current return rod; (b) B-H curve from ringdown test; and (c) evidence of increasing output voltage. (c)

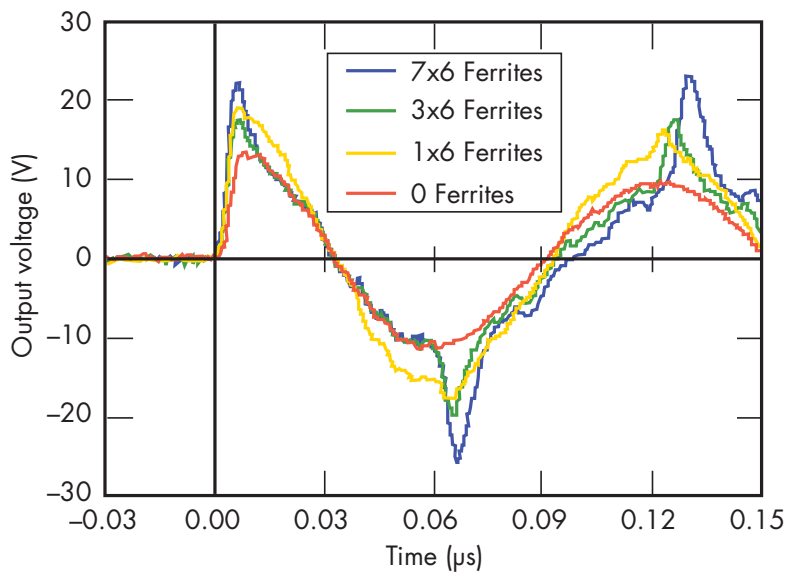




\section{Multipoint Arc Initiation}

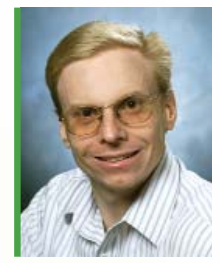

For more information contact David Steich (925) 422-6978, steich1@|ln.gov

he idea of this project is to use an array of arcs, rather than exploding bridge wires or exploding foil initiators, to detonate high explosives (HE). The array of arcs creates extreme temperatures and pressures, which should ignite HE using lower energy than conventional means.

For this year, the feasibility of this concept has been demonstrated by the design of a multipoint system with jitter less than $2 \mathrm{~ns}$. Single arc initiation of numerous types of secondary HE was demonstrated prior to the start of this project.

\section{Project Goals}

The goal of this project is to construct a multipoint arc initiator with low pointto-point jitter.

\section{Relevance to LLNL Mission}

Initiation of HE using lower energies has relevance to numerous DoD and DOE applications. Most important, lowering the energy required for reliable detonation could enable less sensitive HE to be ignited using a given energy. Initiating less sensitive HE has universal relevance to safety improvement.

\section{FY2004 Accomplishments and Results}

A fireset has been built for the initial laboratory testing of multipoint initiators. The fireset consists of a capacitor with a bleed resistor, a spark-gap switch with a trigger board, and a current viewing resistor. The current generation fireset is capable of being charged to $12 \mathrm{kV}$. The charging voltage must be supplied by an external power supply. The capacitor's voltage can be monitored with the fireset's voltage monitor output. The fireset is triggered using a $40-\mathrm{V}$ signal provided by a Stanford delay generator.

A variety of capacitors have been designed and built with capacitances ranging from 300 to $2000 \mathrm{pF}$. These different capacitors will be used to minimize the total circuit energy required for initiation.

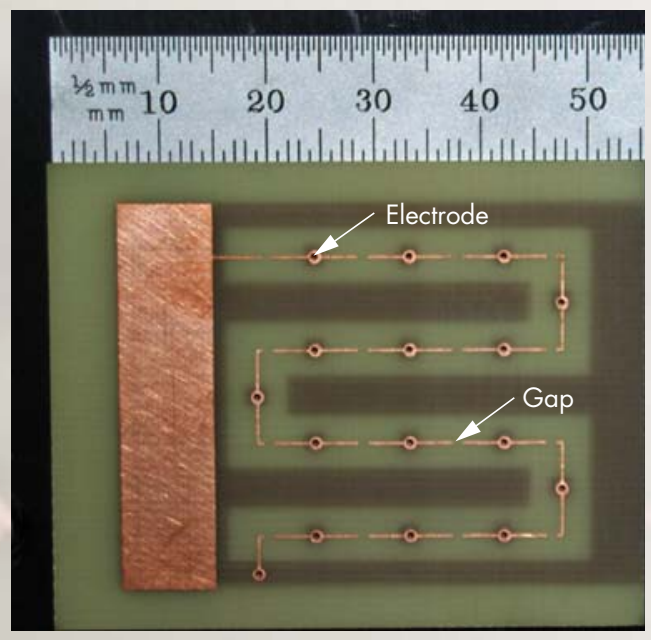

Figure 1. Initiator board.

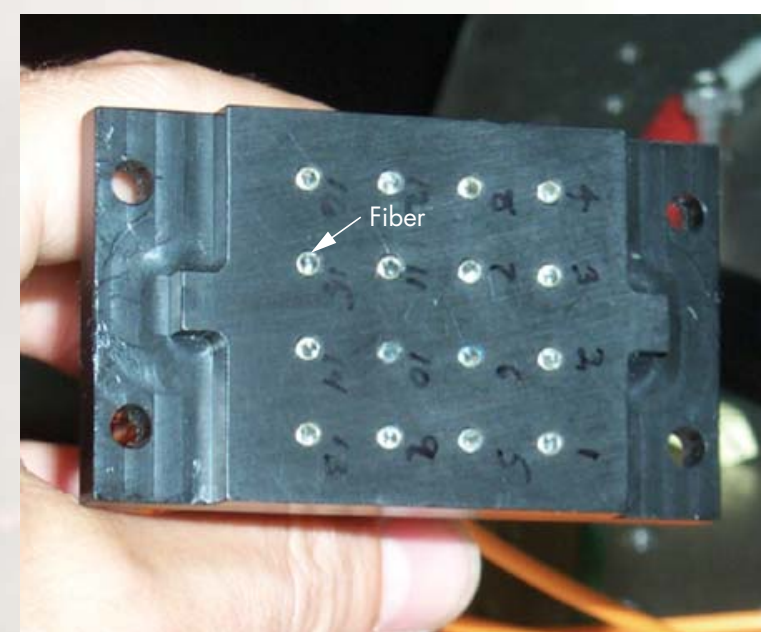

Figure 2. Fiber optics. 
Initiator boards have been built using printed circuit board technology (Fig. 1). In the first series of tests, sixteen-point boards with gap distances of 30, 50, 70 and 90 mils are being studied.

Each electrode is connected to ground through a resistor. The optimal resistance value is under investigation. If the resistance is too low, the capacitor's energy will be depleted before the entire sequence of gaps breakdown. If the resistance is too large, the arc will not be sustained and, potentially, there will be arcing over the resistor to ground.
A fixture has been constructed to house the initiator boards and hold the fiber optics, which view the gaps.

Arc light is transmitted through the fiber optics shown in Fig. 2, and it is imaged onto a streak camera (Fig. 3). The streak camera records this light as well as a timing signal. With the first set of experiments, we have achieved an eight-point system with less than $2.3 \mathrm{~ns}$ of jitter. The jitter may be lower than this, but we are limited in accuracy of the jitter measurement by the camera's sweep speed.

Figure 4 shows the streak record for the eight-point system.

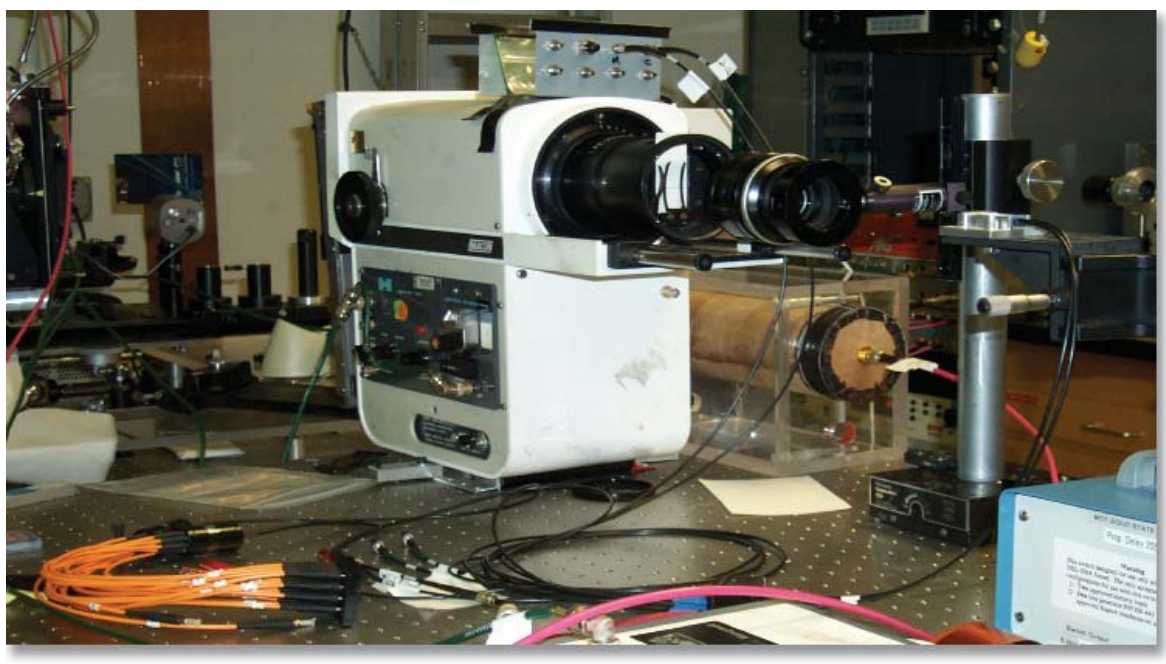

Figure 3. Streak camera and fiber.

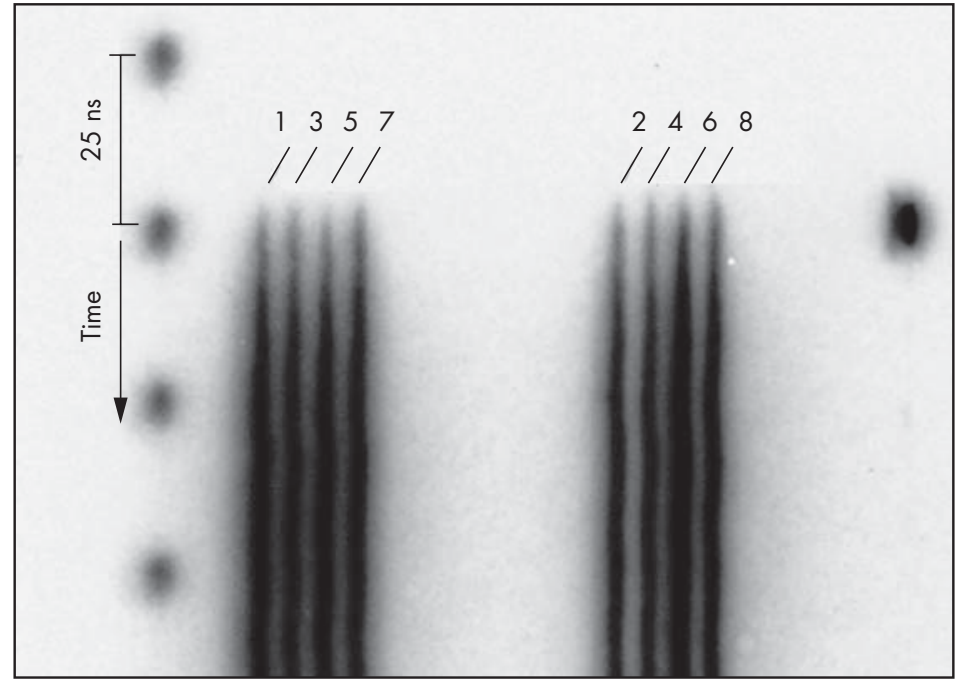

Figure 4. Streak record. The spots on the left are laser pulses spaced at $25 \mathrm{~ns}$; the arrow on the left gives the direction of time. The arcs begin in the faint top region. Eight streaks show the timing for the eight arcs. 


\section{Nano-Mechanical Characterization Tools for In-Situ Deformation and Morphology of Materials}

W e are providing mechanical characterization tools for nano- and micromechanical experimental data in support of multiscale material modeling efforts.

\section{Project Goals}

Our goals are to produce a suite of nano- and micro-mechanical deformation test stages, with various load ranges that can be inserted into a scanning electron microscope (SEM), transmission electron microscope (TEM), atomic force microscope (AFM), and a desktop video system for extreme imaging and surface topography during precision controlled deformation. In addition to the deformation stages, we have a variety of new nano- and micro-tensile/fracture test specimens and grips.

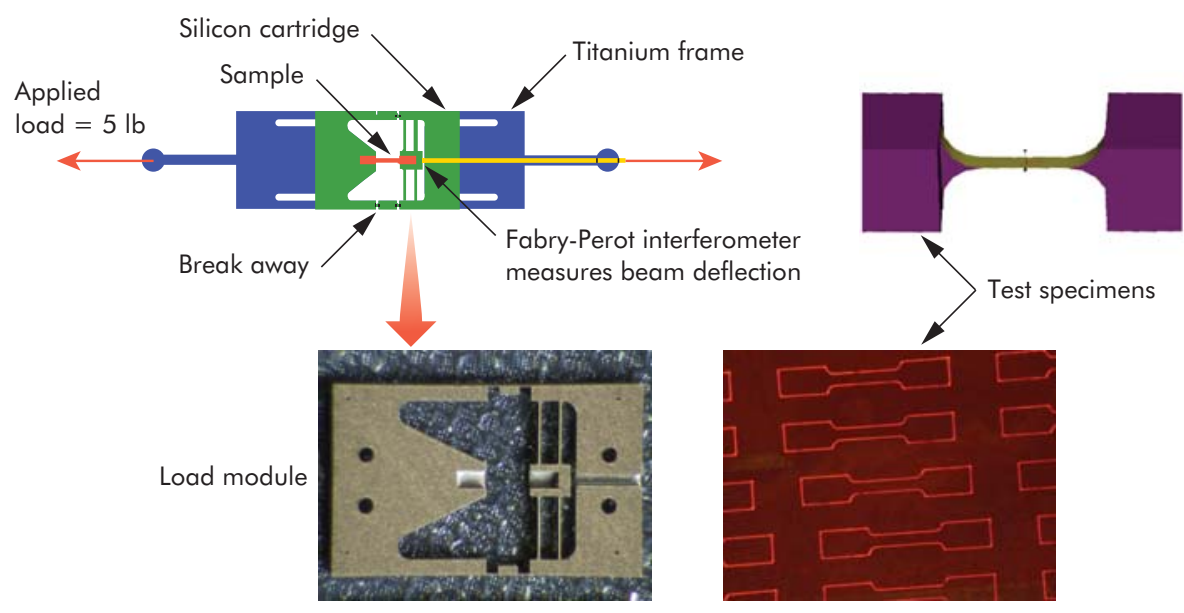

Figure 1. TEM loading stage and specimen. This system uses an external loading frame to deform the test specimen with loads up to $0.0005 \mathrm{lbs}$. The loading frames are fabricated at LLN's Center for Microtechnology and Nanotechnology.

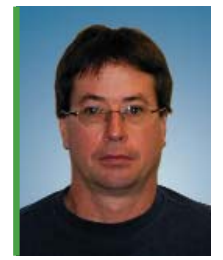

For more information contact Scott Groves

(925) 422-1331, groves2@\|nl.gov

\section{Relevance to LLNL Mission}

These tools allow one to map out the local in situ morphology and inter-granular, inter-particulate, or inter-molecular deformation fields in materials that are subjected to local mechanical stresses. Nanoscale stress-strain data would be obtained which would allow the characterization of localized constitutive models. Load/deformation fields in the vicinity of crack tips in materials could be used to characterize and validate various localized fracture mechanics models. In addition, the small-scale deformation stages can be used to characterize the strength of micro- and nanoscale reinforcements, small samples of material where traditional large specimens cannot be obtained, and the adhesive bond strength of small joints and miniaturized components. The stages have also been reconfigured to study the mechanical properties of the NIF target capsules.

An accurate understanding of the relations between material morphology, deformation, and fracture will provide critical information for enhancing our ability to model the macroscopic response of all materials in general. 


\section{FY2004 Accomplishments and Results}

Four deformation stages have been fabricated, with progressively higher full-scale load ranges from $0.0005 \mathrm{lbs}, 10 \mathrm{lbs}$, $200 \mathrm{lbs}$, and $1000 \mathrm{lbs}$.

Our smallest stage allows deformation imaging using the TEM (Fig. 1). At this imaging level one will be able to see actual dislocation movements. The test specimen used in this extremely small device has a gage section that is $0.2 \mu \mathrm{m}$ thick $\times 100 \mu \mathrm{m}$ wide, which requires focused ion beam machining.

Figure 2 shows our SEM-compatible stages and current tensile specimen. This specimen has a gage section of 0.02 in. thick $\times 0.04 \mathrm{in}$. in width. Twenty of these specimens could be machined from a single dime. At this imaging level, one will be able to see inter-granular deformation fields.

Due to popular demand, a slightly larger test system was created to study material response as well as miniaturized components (Fig. 3). This system has a 1000-lb capacity and uses a video microscope $(15 \times$ to $1000 \times)$ for imaging.
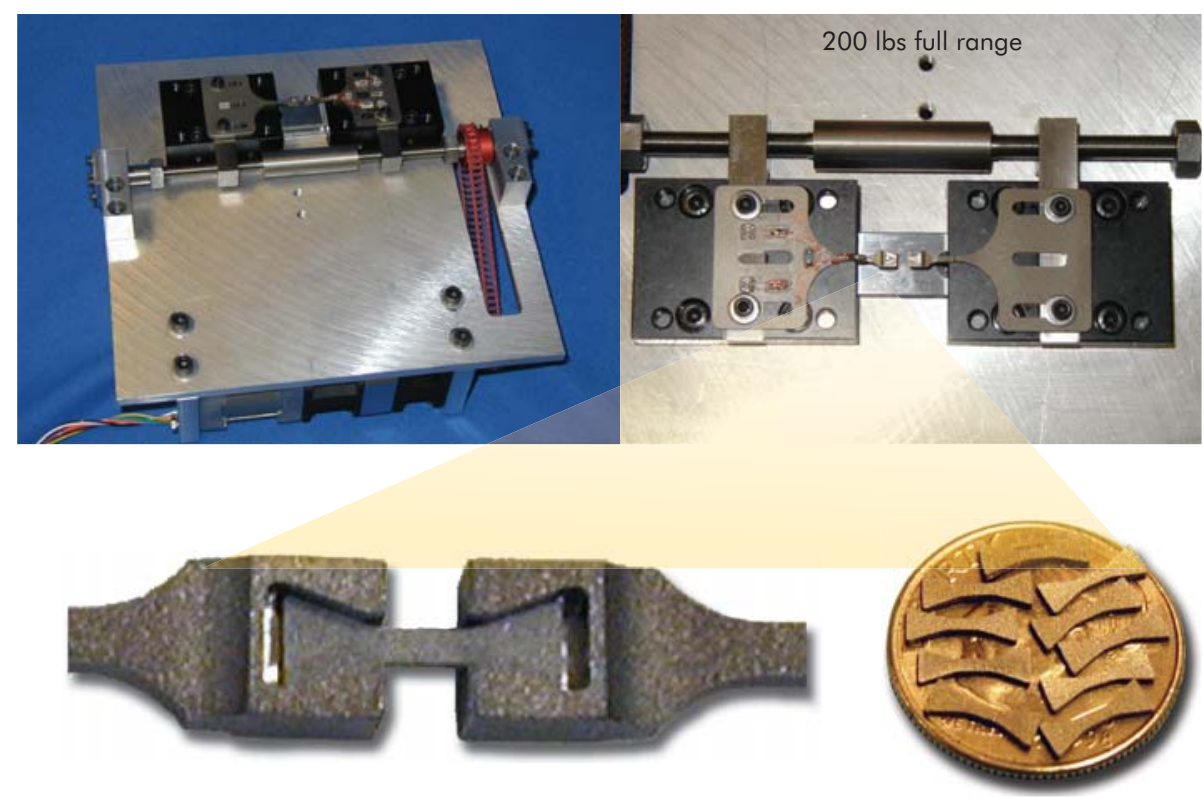

Figure 2. . SEM micro-stage with 1 in. of motion, 000002 in. resolution, and 200-lb force capacity. Test specimen is shown relative to a dime. Specimens are machined with wire EDM.

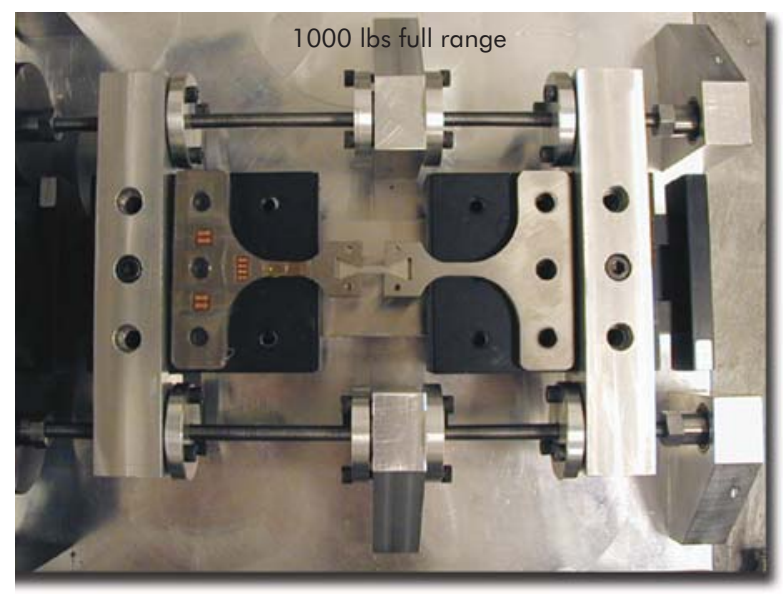

Figure 3. Desktop video micro-stage with 1000-tb force capacity, and tensile test specimen. Specimen is $1.0 \mathrm{in}$. long $\times 0.10 \mathrm{in}$. wide $\times 0.04$ in. thick. Specimens are machined with wire EDM or standard milling practices. 


\section{Solid-State Replacements for Hydrogen Thyratrons}

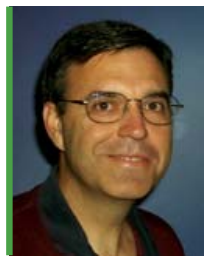

For more information contact Phillip A. Arnold (925) 423-8084, arnold3@|lnl.gov

\section{Project Goals}

Our goals for FY2004 include: 1) evaluating promising commercially-available solid-state devices to prepare guidelines for future pulse-power switching applications in engineering; 2) evaluating device capabilities and limitations as they affect the ability to effectively replace thyratrons in high-power applications; 3) evaluating multiple device types to identify cost-effective solutions for gas-switch replacement; and 4) demonstrating full functionality and lifetimes in a representative LLNL application.

\section{Relevance to LLNL Mission}

Enhancing this expertise will be beneficial to existing and future programs that require high-power switches. LLNL has many needs for high-current/high-energy capacitive discharge units, including magnetic flux compression generators; flashlamp banks and Pockels cell drivers for lasers and NIF; pulsed high-field magnets; EM launchers/rail guns; and compact electric power conversion.

\section{FY2004 Accomplishments and Results}

We have constructed an interlocked high-voltage test-stand with a full suite of electrical diagnostics for evaluating solidstate devices. We have also fielded a data acquisition system (Fig. 1) for efficiently capturing and analyzing waveforms; procured and evaluated multiple families of solid state devices (Fig. 2); created laser diode driver circuitry for light-triggered thyristors that will be directly applicable to new, higher power devices; demonstrated device functionality in existing LLNL pulse-power application; documented performance information for future solidstate switches; tested a light-activated thyristor trigger system/fault detection 
system; evaluated magnetic-assisted switching of thyristors; explored series and parallel operation of high-power thyristors; operated solid-state switches in grounded and off-ground configurations; operated solid-state switches in linear (resistive) and nonlinear (plasma) loads; investigated the effects of high currents and high (di/dt)s on device lifetimes; and investigated failure mechanisms in solidstate devices.

Figure 3 compares solid-state vs. thyratron system complexity.

\section{Related References}

1. Erickson, R. W., and D. Maksimovic, Fundamentals of Power Electronics, Kluwer Academic Publishers, United Kingdom, 2001. 2. Williams, B.W., Power Electronics: Devices, Drivers, Applications and Passive Components, McGraw-Hill, New York, 1992.

3. Kassakian, J., M. Schlecht, and G. Verghese, Principles of Power Electronics, Addison-Wesley, Reading, Massachusetts, 1991.

4. Heumann, K., Basic Principles of Power

Electronics, Springer-Verlag, Germany, 1986.

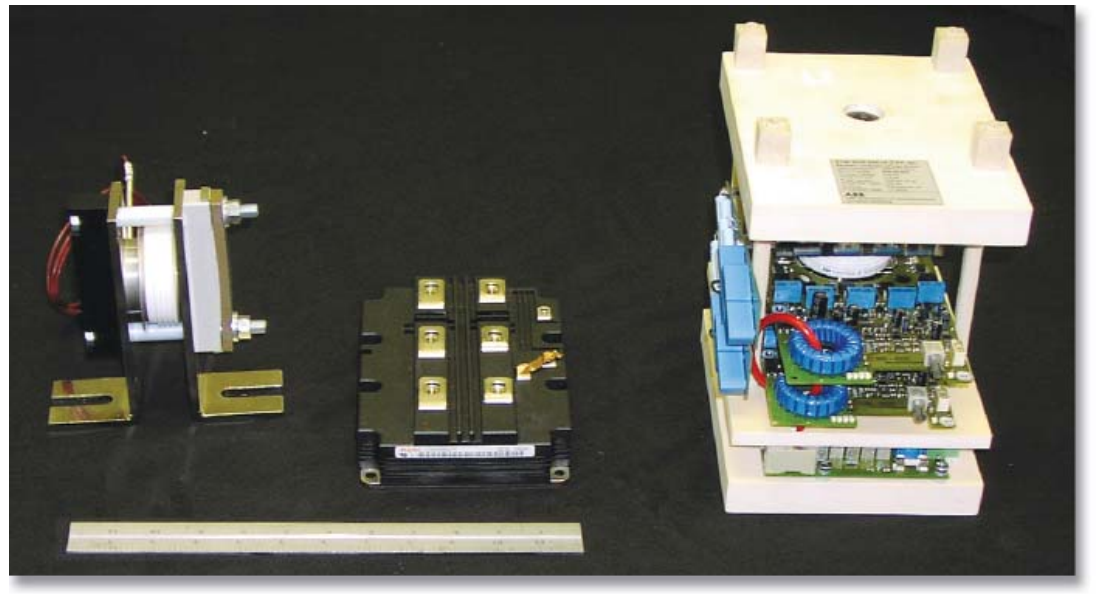

Figure 2. Solid-state devices tested and evaluated. From left to right: Eupec phase-control SCR (light-triggered thyristor); Eupec IGBT (6500-V power bricks); $A B B$ inverter-grade reverse-conducting thyristor stack (pulse-power thyristor).
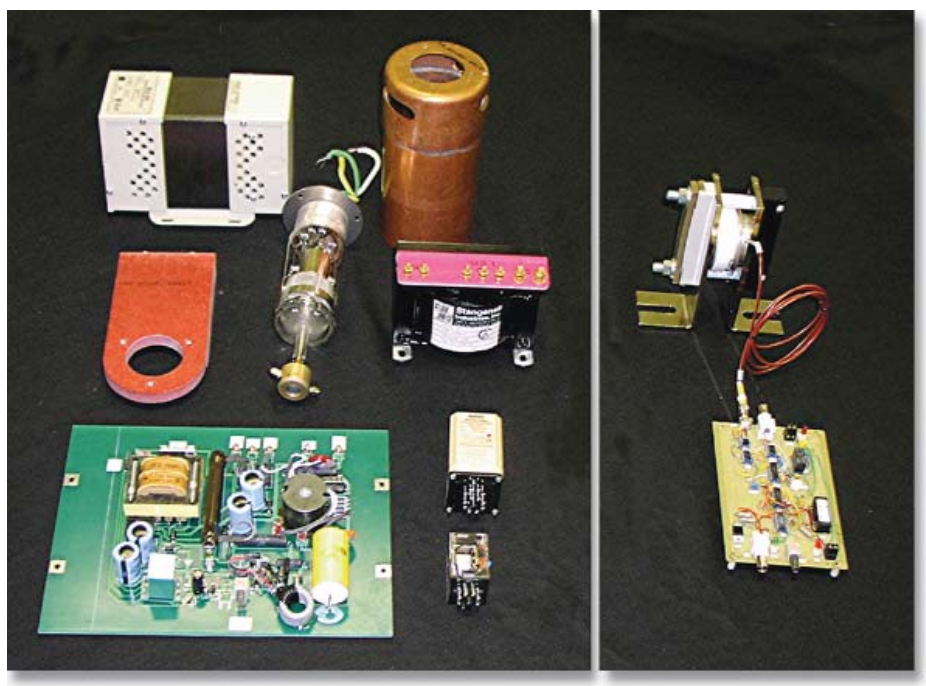

\section{FY2005 Proposed Work}

We propose to evaluate new, higher current, higher (di/dt) devices beyond manufacturers' ratings to determine suitability for pulse-power operation; test operate at higher current and short pulse duration; determine device limitations and/or failure modes; verify that our trigger system can be used with new devices; and further investigate series/parallel switching issues to obtain higher performance.
Figure 3. Solid-state vs. thyratron system complexity. Left side displays thyratron and required ancillary equipment. Right side shows an SCR and driver that replaced the thyratron in proof-of-principle testing. Solidstate switch and driver represent a 75\% reduction in cost with a significant reduction in parts count and volume. 


\section{Transient Sampling}

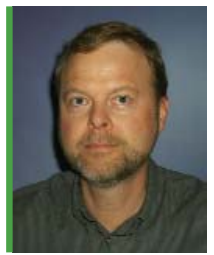

For more information contact Craig S. Halvorson (925) 422-6580, halvorson1@\|nl.gov

any physical phenomena studied at LLNL exhibit complex dynamics that require picosecond or faster temporal resolution. The fastest available commercial oscilloscopes have a resolution on the order of $35 \mathrm{ps}$. One way to achieve faster resolution is to use the optical portion of the electromagnetic spectrum, which has an inherent bandwidth several orders of magnitude greater than microwave electronics.

The transient sampling data recorder works by converting an electrical signal into an optical signal, which is injected into a recirculating fiber loop. Each time the signal circulates in the loop, some of the signal is picked off and recorded using a sampling oscilloscope. Sampling oscilloscopes have much greater bandwidth than storage oscilloscopes, but require repetitive signals. After being sampled, the signal is regenerated using an erbium-doped fiber amplifier (EDFA), and injected back into the loop. The process is repeated a few thousand times, and the sampling oscilloscope builds a detailed record of the transient signal. The apparatus is shown in Fig. 1.

\section{Project Goals}

The goals of the project are to build a high-bandwidth transient sampling data recorder, characterize it, and obtain a quantitative understanding of the technology.

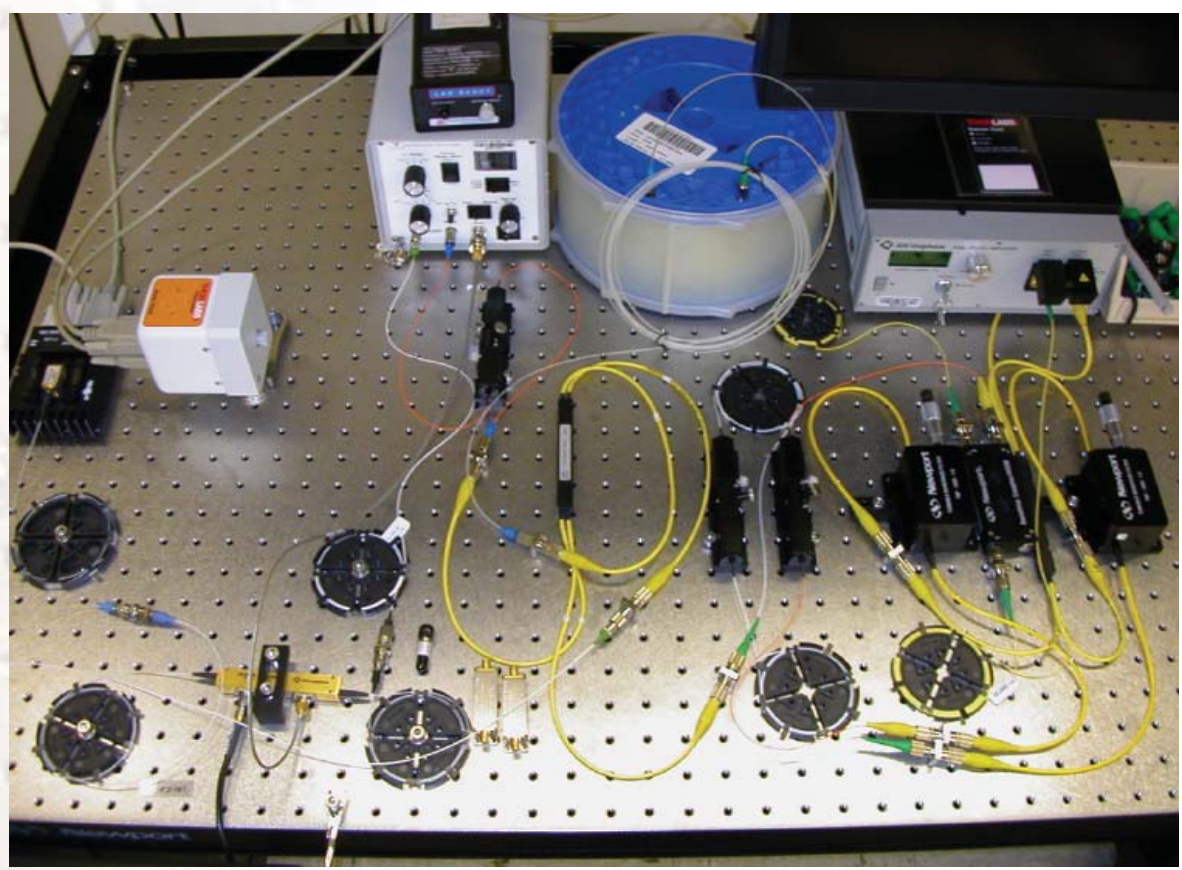

Figure 1. Transient sampling apparatus. 


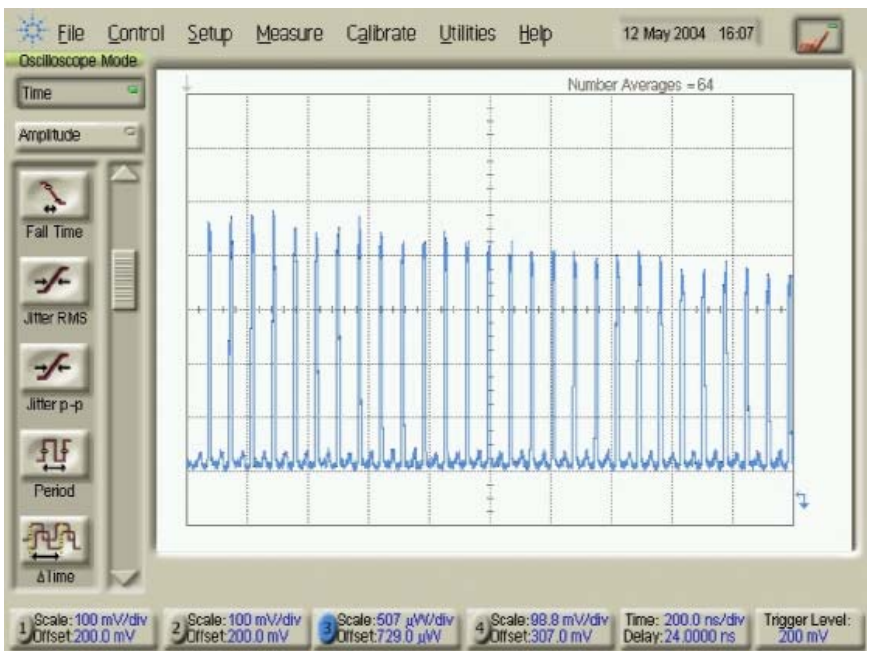

Figure 2. Gain clamping results in multiple signal replicas.

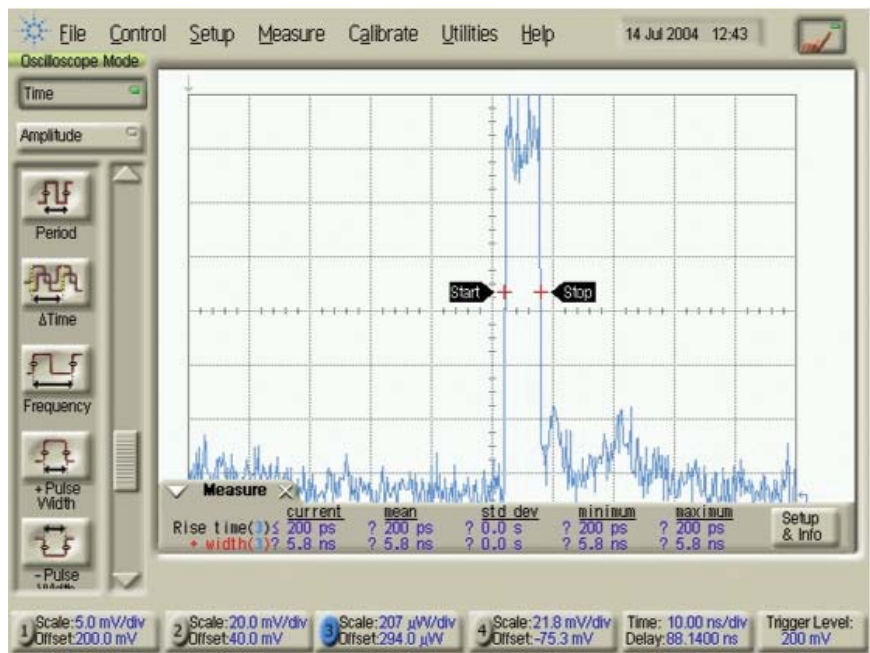

Figure 3. Transient square pulse recorded using the gain clamped apparatus, with 499 pulse replicas. The post-pulse noise is due to timing inaccuracies.

\section{Relevance to LLNL Mission}

High-bandwidth transient data recorders have several applications at LLNL. The high bandwidth could reveal previously unresolvable structure in the reaction history of exploding nuclear devices. Advanced diagnostics for NIF require ps resolution to follow the evolution of complex processes driven by ns and ps laser pulses.

\section{FY2004 Accomplishments and Results}

Several transient sampling data recorders were built and tested, beginning with simple proof-of-concept configurations. The injection of a transient signal was found to alter the gain dynamics of the amplifier, and this led to a rapid reduction of pulse replica amplitude. The addition of a gain clamp resulted in constant pulse amplitudes. Gain clamping an EDFA involves lasing (from noise) at one wavelength, while amplifying on another wavelength. When a transient signal is amplified, photons are removed from the lasing channel, and the amplifier gain remains constant. Gain clamping has allowed several thousand accurate pulse replicas to be created from one signal pulse (Fig. 2).

The transient sampling data recorder was tested using a one-shot square pulse. The data is shown in Fig. 3.

\section{Related References}

1. Kringlebotn, J., P. Morkel, et al., "Amplified Fibre Delay Line with 27000 Recirculations," Electron. Lett., 28, (201), 1992.

2. Zirngibl, A., "Gain Control in Erbium-Doped Fibre Amplifiers," Electron. Lett., 27, (560), 1991. 3. Sun, Y., J. L. Zyskind, et al., "Average Inversion Level, Modeling, and Physics of Erbium-Doped Fiber Amplifiers," IEEE Jour. Sel. Topics Quant. Electr., 3, (991), 1997.

\section{FY2005 Proposed Work}

In FY2005, we will study the capabilities of transient-sampling technology. Any instrument adds distortion to signals, and this is especially true of highbandwidth instruments. Chromatic dispersion, polarization mode dispersion, and component response times can all add distortions to signals recorded using transient sampling. By measuring these sources of distortion, an optimized data recorder can be created. Perhaps more importantly, the fundamental advantages and limitations of transient-sampling technology can be understood.

Ultimately, noise will limit the number of usable pulse replicas that may be generated, and that in turn will limit either the record length or the temporal resolution. It is not clear how many usable pulse replicas can be produced. If the number of replicas could be increased by another order of magnitude, the performance advantage of this type of data recorder could become compelling in many applications.

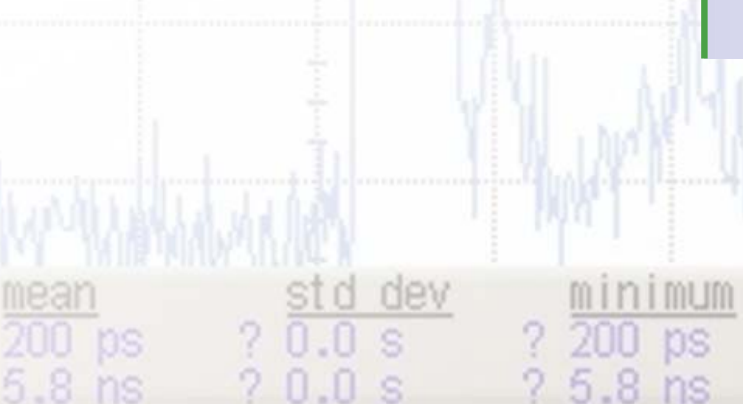




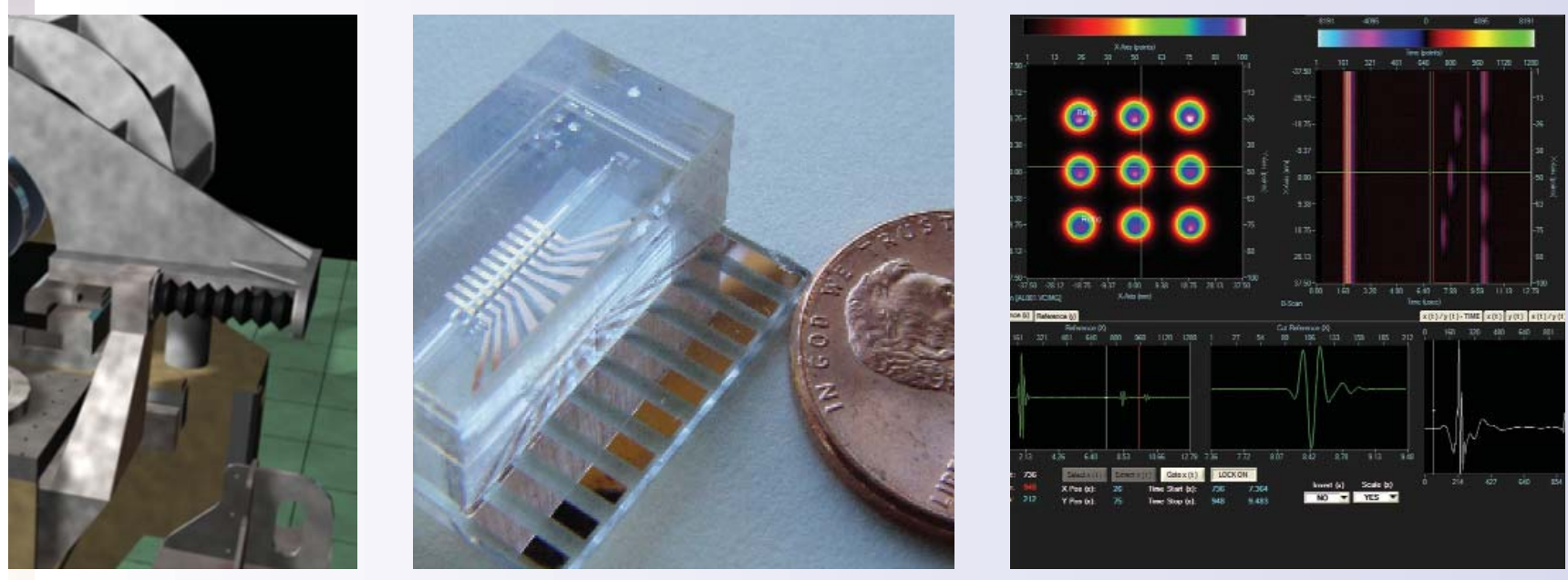

Author Index 


\section{Authar Index}

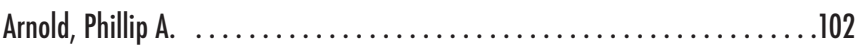

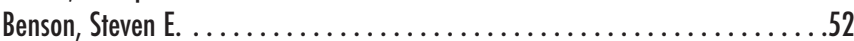

Blessing, J. Steven ................................... 88

Bowers, Joel ......................................... 74

Carrano, Carmen J. .................................. 86

Chambers, David H. .................................... 10

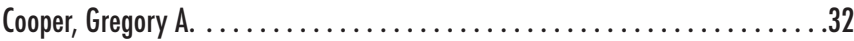

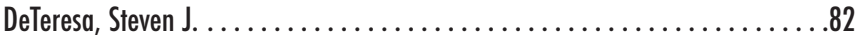

Dowla, Farid U. ...................................... 72

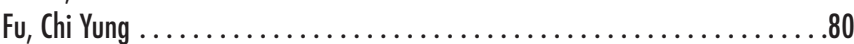

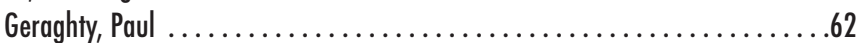

Glascoe, Lee Greer ....................................24

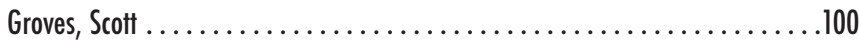

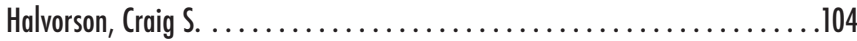

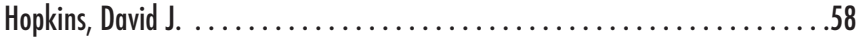

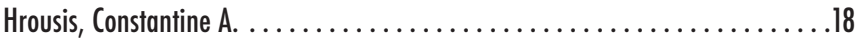

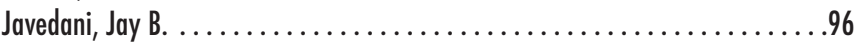

Jones, Erik David ..................................94

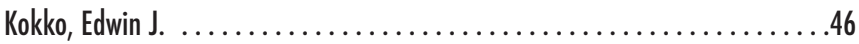

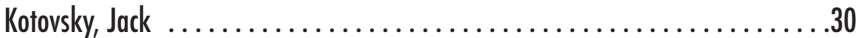

Kroll, Jeremy J. ..................................60

Leach, Richard R., Jr. ............................. 70

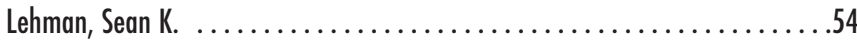

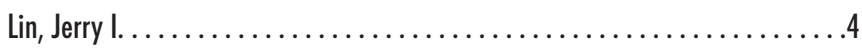

Loomis, Michael D. .......................................12

Malba, Vincent ....................................... 42

Masica, Kenneth M. ...................................68

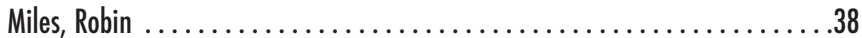

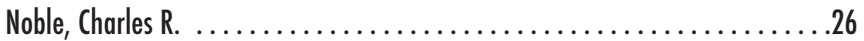

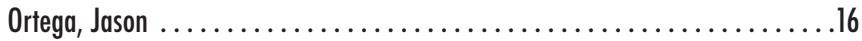

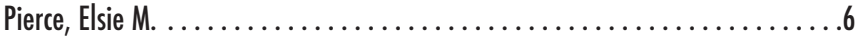

Pocha, Michael D. .................................... 40

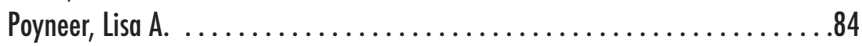

Puso, Michael A. .....................................

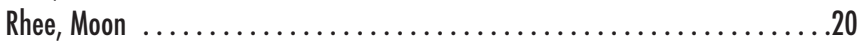

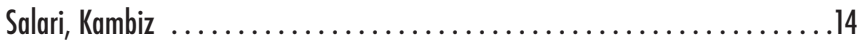

Schneberk, Daniel J. ................................48

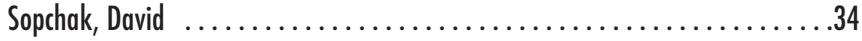

Spiridon, Alex .......................................90

Steich, David .................................. 98

Strauch, Mark ...................................66

Vercelli, Thomas M. .................................. 78

Waters, Amy M. ....................................... 50

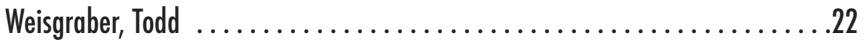

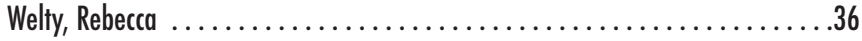

Zaleski, Tania ..................................... 


\section{Manuscript Date April 2005 \\ Distribution Category UC-42}

This report has been reproduced directly from the best available copy.

Available for a processing fee to U.S. Department of Energy and its contractors in paper from

U.S. Department of Energy

Office of Scientific and Technical Information

P.O. Box 62

Oak Ridge, TN 37831-0062

Telephone: (865) 576-8401

Facsimile: (865) 576-5728

E-mail: reports@adonis.osti.gov

Available for sale to the public from

U.S. Department of Commerce

National Technical Information Service

5285 Port Royal Road

Springfield, VA 22161

Telephone: (800) 553-6847

Facsimile: (703) 605-6900

E-mail: orders@ntis.fedworld.gov

Online ordering: http://www.ntis.gov/products/

Or

Lawrence Livermore National Laboratory

Technical Information Department's Digital Library

http://www.llnl.gov/library/

This document was prepared as an account of work sponsored by an agency of the United States Government. Neither the United States Government nor the University of California nor any of their employees, makes any warranty, express or implied, or assumes any legal liability or responsibility for the accuracy, completeness, or usefulness of any information, apparatus, product, or process disclosed, or represents that its use would not infringe privately owned rights. Reference herein to any specific commercial products, process, or service by trade name, trademark, manufacturer, or otherwise, does not necessarily constitute or imply its endorsement, recommendation, or favoring by the United States Government or the University of California. The views and opinions of authors expressed herein do not necessarily state or reflect those of the United States Government or the University of California, and shall not be used for advertising or product endorsement purposes.

This work was performed under the auspices of the U.S. Deparment of Energy by the University of California, Lawrence Livermore National Laboratory under Contract W-7405-Eng-48.

ENG-04-0103-AD 
ENGINEERING LLNL

Lawrence Livermore National Laboratory

University of California

P.0. Box 808, L-151

Livermore, California 94551

http://www-eng.llnl.gov/ 